\title{
Finite-dimensional approximations of quantum systems and Connes' embedding conjecture
}

\author{
Von der Fakultät für Mathematik und Physik \\ der Gottfried Wilhelm Leibniz-Universität Hannover \\ zur Erlangung des Grades \\ Doktor der Naturwissenschaften \\ Dr. rer. nat.
}

genehmigte Dissertation von

Dipl.-Phys. Volkher Bernhard Scholz

geboren am 12.08.1983 in Siegen 
Referent: Prof. Dr. Reinhard F. Werner

1. Korreferent: Prof. Dr. Michael M. Wolf

2. Korreferent: Prof. Dr. Vern Paulsen

Tag der Promotion: 30. Januar 2012 


\section{Zusammenfassung}

Das Ziel dieser Arbeit ist ein besseres Verständnis der Bedingungen wann und wie allgemeine Quantensysteme durch solche mit einer endlichen Anzahl von Freiheitsgeraden approximiert werden können. Im Verlauf der Arbeit finden wir einen engen Zusammenhang dieser physikalischen Frage mit dem Einbettungsproblem von Alain Connes aus der Theorie der schwach abgeschlossenen Teilalgebren von beschränkten Operatoren auf Hilberträumen. In unseren Studien konzentrieren wir uns auf den Fall von zwei unabhängigen Parteien, die beide auf einem gemeinsamen Quantensystem agieren. Wenn beide zwischen endlich vielen Messungen mit jeweils endlich vielen Ausgängen als mögliche Quantenoperation auswählen können, kommen wir zu dem Problem von Tsirelson. Dieses fragt nach der Äquivalenz von verschiedenen mathematischen Modellierungen dieser Situation, einerseits durch Operatoren von Tensorproduktform, wobei der gemeinsame Hilbertraum beider Parteien aus dem Tensorprodukt der einzelnen Hilbertschen Räume besteht, verglichen andererseits mit der weniger restriktiven Annahme, dass die Messoperatoren auf beiden Seiten kommutieren und der gemeinsame Hilbertraum nicht notwendigerweise von Tensorproduktform ist. Nach der Formulierung des Tsirelsonschen Problems in der Sprache von Operatorsystemen finden wir einen engen Zusammenhang mit dem Einbettungsproblem von Alain Connes, dessen positive Lösung eine eben solche des Tsirelsonschen Problem implizieren würde. Wir setzen unsere Arbeit fort mit dem Studium der Bedingungen für die Existenz bestimmter endlichdimensionaler Approximationen von allgemeinen Quantensystemen, welche modelliert werden durch Operatorsysteme. Dabei hilft uns eine Formulierung des Conneschen Problems in der Sprache der Operatorsysteme. Zur weiteren Untersuchung führen wir den Begriff von Ultraprodukten von Operatorsystemen ein und erläutern den Zusammenhang mit Approximationseigenschaften von Quantensystemen. Wir beschließen die Arbeit mit der Untersuchung zweier verschiedener Begriffe von Approximierbarkeit, beide motiviert aus informationstheoretischen Überlegungen. 



\section{Summary}

This thesis studies the requirements for and implications of the existence of finite-dimensional approximations of quantum systems with an infinite number of degrees of freedom. We find a close relationship between these physical questions and the embedding problem of Alain Connes, appearing in the study of weakly-*-closed subalgebras of bounded operators on Hilbert spaces, also known as von Neumann algebras. We are mainly interested in the bipartite scenario known from quantum information theory, where two independent parties act on a joint physical system. These actions are physical transformations, for example measurements with a finite number of outcomes. The later example of physical transformations is used in stating Tsirelson's problem, which asks whether the modeling of bipartite situations using the usual approach of tensor product of Hilbert spaces compared to the situation of only commuting observables leads to the same correlation tables. We model this abstract situation using the theory of operator systems and show the connection to Connes' embedding problem. After studying this abstract physical situation, we use the language of operator systems to elaborate on the requirements for the existence of finite-dimensional approximations for general quantum systems and discuss their relation to concepts from quantum field theory. We furthermore introduce the concept of ultraproducts of operator systems and use it to study the implications of two different kinds of finite-dimensional approximations. Both notions of which are motivated by information theoretic considerations.

Schlagwörter: Unendlichdimensionale Quantensysteme, Operatorsysteme, $\mathrm{C}^{*}$-Algebren Catchword: Infinite-dimensional quantum systems, operator systems, $\mathrm{C}^{*}$-algebras 



\section{Danksagung}

$\mathrm{Zu}$ allererst bedanke ich mich bei meinem Betreuer Prof. Dr. Reinhard F. Werner, der mein Interesse für das Thema geweckt hat, und mir in allen wissenschaftlichen Fragen immer mit seinem außerordentlichem Wissen sowie Rat und Tat zur Seite stand. Ich lernte von ihm die Schönheit algebraischer Argumente, und das man nie die physikalische Intuition verlieren sollte. Er ermöglichte mir viele Konferenzteilnahmen, half mir in karrieretechnischen Fragen und ließ mir allen Freiraum den ich haben wollte. Ich danke ihm dafür von ganzem Herzen.

Ich danke allen meinen Koautoren, insbesondere den Herren Andre Ahlbrecht, Mario Berta, Fabian Furrer, Marius Junge, Carlos Palazuelos und Albert Werner. Es war eine Ehre und eine große Freude mit Ihnen zu arbeiten. Den Herren Furrer und Berta danke ich für viele anregende Diskussionen zur Informationstheorie und viele freudige Stunden in Zürich und Hannover. Marius Junge danke ich insbesondere dafür, mir die Verbindung des Tsirelsonschen Problems mit der Vermutung von Kirchberg aufzuzeigen, und mein Interesse für Ultraproduktkonstruktionen zu wecken.

Ich danke meinen beiden Büropartner Andre Ahlbrecht und Albert Werner für ein schöne, angenehme und gleichzeitig anregende und produktive Arbeitsatmosphäre. Ein besseres Arbeitsklima kann man sich nicht wünschen, und ihre Unterstützung in allen Lebenslagen hat außerordentlich zur Vollendung dieser Arbeit beigetragen. Es war eine Zeit gefüllt mit tollen Diskussionen, und es stehen noch viele wissenschaftliche Projekte an, die in diesem Büro ihren Ursprung haben.

Ich bedanke mich bei allen Mitgliedern der Quanteninformationstheoriegruppe in Braunschweig und Hannover und insbesondere bei Cornelia Schmidt und Wiebke Möller, die mich in allen administrativen Dingen immer hervorragend unterstützt haben, auch wenn das bei meiner Schusseligkeit sicherlich manchmal sehr schwer war. Bei beiden bedanke ich mich insbesondere auch für die angenehmen Gespräche über die Widrigkeiten des Lebens. Besonderen Dank verdienen auch Fabian Furrer, Dr. Michael Bremner und Albert Werner für das Korrekturlesen dieser Arbeit. Bedanken möchte ich mich insbesondere auch bei den Herren Professoren Tobias Osborne und Michael Wolf für unzählige anregende wissenschaftliche Diskussionen sowie ihrer herzlichen und sehr hilfreichen Beratung in Karrierefragen.

Diese Doktorarbeit wäre nicht möglich gewesen ohne mein Studium der Physik an der Universität Erlangen-Nürnberg. Für ihre fortwährende Unterstützung möchte ich insbesondere den Herren Professoren Hajo Leschke, Norbert Lütkenhaus und Paul Müller meinen tiefen Dank ausdrücken. Nicht zu vergessen ist auch die phänomenale Cafetenrunde, deren Mitglieder mir auch nach meinen Studium immer in allen Lebenslagen und nicht nur in wissenschaftlichen Fragen mit Rat und Hilfe zur Seite standen. Besonderes zu erwähnen sind dabei die Doktoren Tobias Moroder und Wolfgang Mauerer, dem ich insbesondere auch für seine Hilfe in Sachen $\mathrm{IAT}_{\mathrm{E} X}$ danke, sowie Bettina Heim und Michael Enzelsberger.

Ich bedanke mich bei meinen Freunden vom Akademischen Reitclub Hannover, für ihre andauernde Unterstützung und die vielen unvergesslichen lustigen Stunden, insbesondere während unserer alljährlichen Hubertusjagd sowie des Weinachtsessens. Mein ganz be- 
sonderer Dank gilt meinem besten Freund Stefan Spahmann, dessen tiefe und herzliche Freundschaft mir in unzähligen Lebenslagen sehr geholfen hat.

Mein tief empfundener Dank gilt auch meiner großen Liebe Ines, die mich immer und überall unterstützt, und mir die Kraft gab diese Arbeit zu vollenden. Ihre Liebe baut mich fortwährend auf und gibt mir die Kraft mich voll auf meinen Beruf zu konzentrieren.

Mein allergrößter Dank gilt meiner Familie, meiner Mutter Helga Scholz, meinem Vater Dr. Bernhard Scholz und meinem Bruder Wolfgang Scholz. Meine Freude an der Physik verdanke ich meinem Vater, meine Mutter erweckte in mir das Unternehmertum, und meinem Bruder danke ich für die vielen philosophischen Diskussionen. Ohne sie wäre mein Studium und diese Arbeit nicht möglich gewesen. Ich danke ihnen alle, für alles. 


\section{List of Publications}

The following is a list of my publications finished during my phd, including abstracts. Publications 1, 2 and 4 are in connection with topics discussed in this thesis.

1. Volkher B. Scholz and Reinhard F. Werner, Tsirelson's Problem. Preprint, arXiv:0812.4305, 2008.

The situation of two independent observers conducting measurements on a joint quantum system is usually modeled using a Hilbert space of tensor product form, each factor associated to one observer. Correspondingly, the operators describing the observables are then acting non-trivially only on one of the tensor factors. However, the same situation can also be modeled by just using one joint Hilbert space, and requiring that all operators associated to different observers commute, i.e. are jointly measurable without causing disturbance. The problem of Tsirelson is now to decide the question whether all quantum correlation functions between two independent observers derived from commuting observables can also be expressed using observables defined on a Hilbert space of tensor product form. Tsirelson showed already that the distinction is irrelevant in the case that the ambient Hilbert space is of finite dimension. We show here that the problem is equivalent to the question whether all quantum correlation functions can be approximated by correlation function derived from finite-dimensional systems. We also discuss some physical examples which fulfill this requirement.

2. Marius Junge, Miguel Navascues, Carlos Palazuelos, David Perez-Garcia, Volkher. B. Scholz, Reinhard F. Werner, Connes' embedding problem and Tsirelson's problem. J. Math. Phys. 52, 012102, 2011.

We show that Tsirelson's problem concerning the set of quantum correlations and Connes' embedding problem on finite approximations in von Neumann algebras (known to be equivalent to Kirchberg's QWEP conjecture) are essentially equivalent. Specifically, Tsirelson's problem asks whether the set of bipartite quantum correlations generated between tensor product separated systems is the same as the set of correlations between commuting $C^{*}$-algebras. Connes' embedding problem asks whether any separable $I I_{1}$ factor is a subfactor of the ultrapower of the hyperfinite $I I_{1}$ factor. We show that an affirmative answer to Connes' question implies a positive answer to Tsirelson's. Conversely, a positive answer to a matrix valued version of Tsirelson's problem implies a positive one to Connes' problem.

3. Andre Ahlbrecht, Volkher B. Scholz and Albert H. Werner, Disordered Quantum Walks in one lattice dimension. J. Math. Phys. 52, 102201, 2011.

We study a spin-1/2-particle moving on a one dimensional lattice subject to disorder induced by a random, space-dependent quantum coin. The discrete time evolution is 
given by a family of random unitary quantum walk operators, where the shift operation is assumed to be deterministic. Each coin is an independent identically distributed random variable with values in the group of two dimensional unitary matrices. We derive sufficient conditions on the probability distribution of the coins such that the system exhibits dynamical localization. Put differently, the tunneling probability between two lattice sites decays rapidly for almost all choices of random coins and after arbitrary many time steps with increasing distance. Our findings imply that this effect takes place if the coin is chosen at random from the Haar measure, or some measure continuous with respect to it, but also for a class of discrete probability measures which support consists of two coins, one of them being the Hadamard coin.

4. Bruno Nachtergaele, Volkher B. Scholz, Reinhard F. Werner, Local approximation of observables and commutator bounds. Preprint, arXiv:1103.5663, 2011.

We discuss conditional expectations that can be used as generalizations of the partial trace for quantum systems with an infinite-dimensional Hilbert space of states.

5. Andre Ahlbrecht, Andrea Alberti, Dieter Meschede, Volkher B. Scholz, Albert H. Werner, Reinhard F. Werner, Bound Molecules in an Interacting Quantum Walk. Preprint, arXiv:1105.1051, 2011

We investigate a system of two atoms in an optical lattice, performing a quantum walk by state-dependent shift operations and a coin operation acting on the internal states. The atoms interact, e.g., by cold collisions, whenever they are in the same potential well of the lattice. Under such conditions they typically develop a bound state, so that the two atoms effectively perform a quantum walk together, rarely moving further from each other than a few lattice sites. The theoretical analysis is based on a theory of quantum walks with a point defect, applied to the difference variable. We also discuss the feasibility of an experimental realization in existing quantum walk experiments.

6. Mario Berta, Fabian Furrer, and Volkher B. Scholz, The Smooth Entropy Formalism on von Neumann Algebras. Preprint, arXiv:1107.5460, 2011.

We discuss quantum information theoretical concepts on von Neumann algebras and lift the smooth entropy formalism to the most general quantum setting. For the smooth min- and max-entropies we recover similar characterizing properties and informationtheoretic operational interpretations as in the finite-dimensional case. We generalize the entropic uncertainty relation with quantum side information of Tomamichel and Renner and sketch possible applications to continuous variable quantum cryptography. In particular, we prove the possibility to perform privacy amplification and classical data compression with quantum side information modeled by a von Neumann algebra. From this we generalize the formula of Renes and Renner characterizing the optimal length of a distillable secure finite-key. We also elaborate on the question when the formalism of von Neumann algebras is of advantage in the description of quantum systems with an infinite number of degrees of freedom. 


\section{Inhaltsverzeichnis}

1 Introduction $\quad 5$

2 Mathematical prelude 9

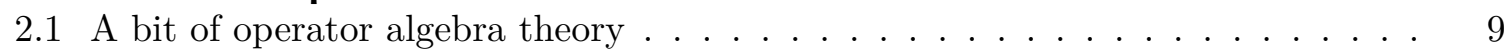

2.2 The conjectures of Connes and Kirchberg . . . . . . . . . . . . . . 18

3 Archimedian ordered vector spaces and Operator Systems 27

3.1 Archimedian order unit spaces . . . . . . . . . . . . . . . . . . 27

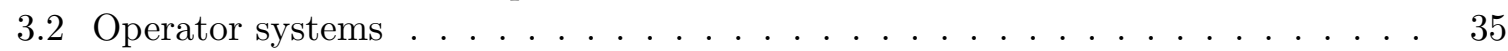

4 Tsirelson's problem and the QWEP conjecture $\mathbf{5 1}$

4.1 The operator system of local measurements . . . . . . . . . . . . . . . 52

4.2 Connecting Tsirelson's problem with Kirchberg's conjecture . . . . . . . . . . 59

$\begin{array}{lll}5 & \text { Tensor products and finite-dimensional approximations } & 67\end{array}$

5.1 An operator system perspective on QWEP . . . . . . . . . . . . . . 67

5.2 Tsirelson's problem revisited . . . . . . . . . . . . . . . . . . . 73

6 Ultraproducts of operator systems and finite-dimensional approximations $\quad 79$

6.1 Ultraproducts of Archimedian order unit vector spaces . . . . . . . . . . . . 80

6.2 Ultraproducts of operator systems . . . . . . . . . . . . . . . . . . 84

6.3 On approximation properties of operator systems $\ldots \ldots \ldots$. . . . . . 94

$\begin{array}{lll}7 & \text { Conclusions and Outlook } & 105\end{array}$ 



\section{Notation index}

\section{General}

\begin{tabular}{|c|c|}
\hline $\mathbb{N}, \mathbb{Z} \mathbb{R}, \mathbb{C}$ & $\begin{array}{l}\text { the sets of positive integers, positive and negative integers } \\
\text { and the sets of real and complex numbers. }\end{array}$ \\
\hline $\mathbb{C}^{n}$ & $\begin{array}{l}\text { complex vector space of dimension } n \text {, equipped with the } \\
\text { canonical scalar product }\langle. \mid .\rangle \text {. }\end{array}$ \\
\hline$\Re e, \Im m$ & real and imaginary part. \\
\hline $\mathcal{H}, \mathcal{K}$ & separable Hilbert spaces. \\
\hline conv & convex hull of elements. \\
\hline span & complex linear span of elements. \\
\hline$\left\{\left|\xi_{i}\right\rangle\right\}_{i \in\{1, \ldots, n\}}$ & orthonormal basis of $\mathbb{C}^{n}$ \\
\hline$\left|\xi_{i}\right\rangle\left\langle\xi_{j}\right|$ & $\begin{array}{l}\text { canonical matrix basis associated to the orthonormal basis } \\
\left\{\left|\xi_{i}\right\rangle\right\}_{i \in\{1, \ldots, n\}} \text {. }\end{array}$ \\
\hline
\end{tabular}

\section{Operator algebras}

$\begin{array}{ll}M_{n} & \text { complex valued matrices of dimension } n \in \mathbb{N} . \\ \mathcal{B}(\mathcal{H}) & \text { the } \mathrm{C}^{*} \text {-algebra of bounded operators on the Hilbert space } \\ \mathcal{H} & \text { unique hyperfinite factor of type } I I_{1} . \\ \mathcal{A}, \mathcal{B} & \mathrm{C}^{*} \text {-algebras. } \\ \mathcal{J} & \text { two-sided ideal in a } \mathrm{C}^{*} \text {-algebra. } \\ \mathcal{A}^{*}, \mathcal{A}^{* *} & \text { dual and bidual of some } \mathrm{C}^{*} \text {-algebra } \mathcal{A} . \\ \mathcal{M}, \mathcal{N}, \mathcal{M}_{*}, \mathcal{N}_{*} & \text { von Neumann algebras and their preduals. } \\ \mathcal{O}^{\prime} & \text { commutant of some set of operators } \mathcal{O} . \\ C^{*}\left(\mathbb{F}_{n}\right) & \text { the universal } \mathrm{C}^{*} \text {-algebra of the free group with } n \text { generators. } \\ \ell_{k}^{\infty} & \text { the finite-dimensional commutative } \mathrm{C}^{*} \text {-algebra of complex } \\ & \text { valued } k \text {-tuples. } \\ *_{n} & \text { the free product of groups or } \mathrm{C}^{*} \text {-algebras, see definition } 4.1 .3 \\ & \text { on page } 55 .\end{array}$




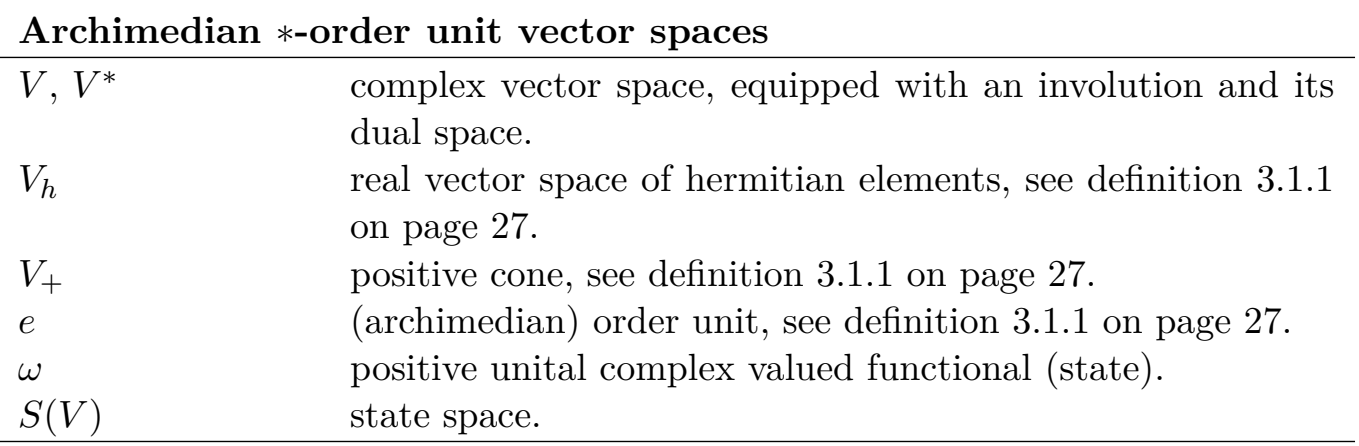

\section{Operator systems and tensor products}

$\begin{array}{ll}S, V, \mathcal{T}, W & \text { operator systems, see definition } 3.2 .1 \text { on page } 36 . \\ C^{*}(S), I(S) & \text { universal algebra and injective envelope, see the discussion } \\ & \text { on page } 45 . \\ & \text { the operator system of } n \text { measurements with } k \text { outcomes } \\ & \text { each, } n, k \in \mathbb{N} \text {, see definition } 4.1 .1 \text { on page } 54 . \\ \otimes_{n, k} & \text { minimal and maximal operator system tensor product, see } \\ & \text { definition } 3.2 .4 \text { on page } 42 .\end{array}$

\section{Ultraproducts}

$\begin{array}{ll}\mathcal{I}, \alpha & \text { some index set and an element of } \mathcal{I} . \\ \mathcal{U}, \mathcal{V}, \mathcal{W} & \text { free ultrafilters. } \\ (\mathcal{R})_{\mathcal{U}} & \text { ultrapower of the unique hyperfinite factor of type } I I_{1} . \\ \left(V_{\alpha}\right)_{\mathcal{U}} & \text { ultraproduct operator system, see definition } 6.2 .1 \text { on page } \\ \approx_{(\varepsilon, n)} & 85 . \\ & \text { notion of approximation between maps on operator systems } \\ & \text { see the discussion preceding definition } 6.2 .2 \text { on page } 90 .\end{array}$


Abbreviations

WEP

weak expectation property, see equation (2.7) on page 23 .

QWEP

quotient weak expectation property, see discussion preceding proposition 2.2 .8 on page 25 .

LLP local lifting property, see the discussion preceding definition 3.2 .7 on page 48 .

DCEP double commutant expectation property, see definition 3.2.6 on page 47 .

OSLLP operator system local lifting property, see definition 3.2.7 on page 48.

QWEPCP QWEP complemented operator system, see definition 5.1.1 on page 67.

weak model see definition 6.3 .1 on page 95 .

weak local model see definition 6.3 .2 on page 101 . 



\section{1 \\ Introduction}

The basic entity of quantum information is a two-dimensional quantum system, also called a quantum bit, or qubit. It is the corresponding quantum generalization of its classical counterpart, the bit. Like a bit, it quantifies the information which can be stored in a physical system with two degrees of freedom, albeit now a quantum one. And like bits, qubits are used to quantify the amount of quantum information needed to perform some physical task, or the amount of information which can be transmitted in a given setup using some prescribed helper objects. We usually assume that this amount is finite. That is, we postulate that physical processes can only use and produce a finite amount of information.

Applying this principle to quantum systems, we end up with quantum systems which can be modeled by operations on a finite number of qubits. A qubit is, by the axioms of quantum mechanics, described by a two-dimensional Hilbert space, and their joint systems by the Hilbert space tensor products between single qubit Hilbert spaces. We arrive at quantum systems modeled by finite-dimensional Hilbert spaces. This corresponds to quantum systems having a finite number of degrees of freedom.

But even a single atom is a quantum system with an infinite number of degrees of freedom, having an infinite number of electronic energy levels. Of course, we usually assume that only a finite number of this levels play a significant role in some physical process we want to study. Another example of quantum systems with an infinite number of degrees of freedom are continuous variable systems, or modes of light. Restricting both space and time, we end up with a finite number of frequencies which have to be studied. We are led to the conclusion that in most experimental setups we operate with physical systems which are modeled on infinite-dimensional Hilbert spaces, but effectively physical operations involving this quantum system are described using only a finite number of degrees of freedom. The term "effectively" here means that we suppose that on the ground of finitely many experiments there are no observable differences.

The aim of the present thesis is to study the justifications of doing so. That is, we are interested in the exact requirements for the existence of finite-dimensional approximations of quantum mechanical systems. Especially, we consider bipartite scenarios where each party holds one share of a joint physical state. They both apply physical operations on their 
respective subsystems, and possibly measure at the end. Their general aim thereby being to create correlated data, either of classical or quantum nature, between each other. From the general considerations above, we furthermore demand that the amount of information both hold at the beginning and at the end of the process (in the following such a sequence of steps is also called a protocol) is finite. We want to understand under which conditions the quantum system on one or at both sides, which is used in the successive steps of the protocol, can be "effectively" replaced by a quantum system having only a finite number of degrees of freedom.

In order to describe quantum systems with an infinite number of degrees of freedom, it is often more accurate and mathematically more elegant to follow an algebraic approach based on operator algebras, or more precisely von Neumann and $\mathrm{C}^{*}$-algebras. For example, these kind of formulations have the advantage that physical symmetries can readily be included into the algebraic structure. The existence of finite-dimensional approximations for von Neumann and $\mathrm{C}^{*}$-algebras have been central questions throughout the history of their study, starting with papers of von Neumann and Murray, [64]. They lead to the emergence of the embedding problem of Alain Connes [17] on the structure von Neumann algebras admitting a tracial state, corresponding, roughly speaking, to a quantum state of highest uncertainty. In loose words, it asks for the existence of finite-dimensional approximations for unitary operators.

The embedding problem of Alain Connes is one of the outstanding problems in the theory of operator algebras nowadays. The greatest progress so far has been achieved by Eberhard Kirchberg [55], who obtained a number of reformulations and proved the equivalence of Connes' embedding problem to open questions in $\mathrm{C}^{*}$-algebra theory. In particular, he introduced the notion of $Q W E P \mathrm{C}^{*}$-algebras, and showed that Connes' embedding problem is equivalent to the statement that every $\mathrm{C}^{*}$-algebra belongs to this class. This is also called Kirchberg's conjecture and it is is connected to the existence of certain finite-dimensional approximations. This leads to the conclusion that the justification of finite-dimensional approximations of quantum systems could lead via the solution of Connes' embedding problem.

However, we are in general not interested in the hole observable algebra of some given quantum system. Instead, in each experiment we are let to choose between a finite number of possible operations to apply, and then a finite number of measurements to choose from in order to gain some knowledge about the system in question. This leads to the definition of operator systems, a category of mathematical objects first introduced by Choi and Effros [15]. The systematic study of these mathematical objects only started in recent years, mainly lead by Paulsen and coworkers [71, 51, 52, 27].

In this thesis, we obtain several reformulations of the Connes' embedding problem in terms of operator systems.In rough words, the result of this thesis is the following "metastatement", which is made precise in the following chapters.

If the embedding problem of Alain Connes' has a positive answer, then all physical experiments performed in a bipartite scenario, where at the beginning and at the end both parties hold a finite amount of (quantum) information, can "effectively" modeled using finite-dimensional quantum systems. Conversely, if every quantum system would admit finite-dimensional approximations valid in these cases, then Connes' embedding problem and Kirchberg's conjecture have a positive answer. 
Let us briefly comment on the structure of this thesis. We start with two chapters introducing the relevant concepts, followed by three chapters on the connection between Connes' embedding problem and the existence of finite-dimensional approximations.

Chapter 2 (Mathematical prelude). We start with section 2.1 by providing a short introduction into the theory of von Neumann and $\mathrm{C}^{*}$-algebras, with connection to physical systems. The second section of this chapter introduces the embedding problem of Alain Connes and the equivalent conjecture of Kirchberg in a formal way. Moreover, we list other reformulations, especially those with implications for quantum information theory.

Chapter 3 (Archimedian ordered vector spaces and Operator Systems). The aim of chapter 3 is to provide the reader with a short introduction into the theory of archimedian $*$-ordered unit vector spaces and operator systems, which form a sub-category of the later class. All introductory material is kept shortly, trying to give a self-contained overview. However, the interested reader is pointed for further questions to the literature on the corresponding topics.

Chapter 4 (Tsirelson's problem and the QWEP conjecture). The results part of this thesis starts with chapter 4 , where we investigate the relation between Tsirelson's problem and the Connes' embedding problem. In short, Tsirelson's problem asks for the equivalence of bipartite correlation tables obtained from two parties, applying a finite number of measurements with a finite number of outcomes, and either be modeled using a Hilbert space of tensor product form or only assuming that the observables on both sides do not influence each other, hence commute. We first construct the operator systems describing these abstract situations in section 4.1. In the following section, we show that a positive answer to Connes' embedding problem would imply a positive one to Tsirelson's problem, too. The results are summarized in theorem 4.2.1 and proposition 4.2.6. We also explain the connection to the existence of finite-dimensional approximations in detail.

Chapter 5 (Tensor products and finite-dimensional approximations). After considering abstract physical situations, the next chapter focuses on study of single quantum systems. In section 5.1 we introduce a possible extension of the QWEP class to the category of operator system and study its properties, the main results being summarized in theorems 5.1.2 and 5.1.3. The second section of this chapter studies the properties of this class with respect to the existence of finite-dimensional approximations of some fixed operator system, see for example corollary 5.2.2.

Chapter 6 (Ultraproducts of operator systems and finite-dimensional approximations). The converse statements on the implications of the existence of finite-dimensional approximations requires the development of the notion of ultraproducts of operator systems. This is started in section 6.1 of chapter 6 for the case of archimedian $*$-ordered vector spaces, followed by the application to operator systems in section 6.2 . The main structure theorem is given by theorem 6.2.5, together with proposition 6.2 .3 . The last section 6.3 introduces two kinds of finite-dimensional approximations for operator systems and studies their consequences, summarized in corollary 6.3.4 and proposition 6.3.5. We end with explaining proposition 6.3 .5 from a quantum information theoretic point of view. 
Chapter 7 (Conclusions and Outlook). We conclude with a short summary and a outlook on future topics of research. 


\section{2 \\ Mathematical prelude}

This chapter provides a short introduction into the mathematical concepts used in the following chapters. In the first section we recall basic facts about $\mathrm{C}^{*}$ - and von Neumann algebras and their connection to physics. The aim of the second section is to introduce the conjectures of Connes and Kirchberg, explain their equivalence, and state related questions. For detailed information on the material covered in the first section, see for example the volumes I-III of the series of Takesaki, [83, 84, 85]. Most statements in the second section are nicely reviewed by Ozawa, [69] and in the survey by Carparo, [14].

\subsection{A bit of operator algebra theory}

We begin by introducing norm-closed and weakly closed operator algebras. Why are these objects useful in the study of physical systems? The usual description of a quantum system in quantum information theory is as follows. The basic object for every physical system is a finite-dimensional Hilbert space $\mathcal{H}$ and states are described by density operators. These are linear and positive semi-definite operators $\rho$ from $\mathcal{H}$ to itself with trace equal to one. Evolution is described by completely positive transformations, induced, for example, by a measurement of an observable, or the interaction with another physical system.

Operator algebras in physics. In contrast to quantum information theory, in general quantum theory, especially in quantum field theory, the basic object for every physical system is a von Neumann algebra $\mathcal{M}$ of observables. Based on the usual approach as given above this can be motivated as follows. Starting with a separable Hilbert space $\mathcal{H}^{1}$, it is often the case that the physical system obeys certain symmetry conditions. Such symmetries can be due to the evolution determined by a Hamilton operator (dynamical symmetries) or postulated symmetries of the theory itself (superselection rules [35]). Typical examples are, for instance, Ising spin chains at zero temperature which are translationally invariant, or bosonic systems that are invariant under particle permutation. Such symmetry constraints

\footnotetext{
${ }^{1}$ All Hilbert spaces in this theses are assumed to be separable, unless otherwise stated.
} 
are realized by a representation $\pi$ of the symmetry group $G$ on $\mathcal{H}$, and the requirement that all observables $O$ of the theory are invariant under group actions $g \in G$,

$$
\pi\left(g^{-1}\right) O \pi(g)=O
$$

which is the same as saying that all physical states are invariant. If the group acts reducibly on $\mathcal{H}$, it follows that not all mathematical possible observables can actually be observed. Hence, the set of physical observables form a $*$-subalgebra ${ }^{2}$ of the linear, bounded operators $\mathcal{B}(\mathcal{H})$ on $\mathcal{H}$. If $\mathcal{H}$ is infinite-dimensional, the imposed topological requirements on these subalgebras are, however, more subtle. Take a sequence $O_{i}$ of observables such that $\operatorname{Tr}\left(\rho O_{i}\right)$ is a convergent sequence for all density operators $\rho$ on $\mathcal{H}$. It is then physically reasonable to require that a 'limit' observable $O$ exists which give rise to this value. In other words, we would require that the subalgebra of physical observables is closed with respect to taking expectation values. This topology is usually called the $\sigma$-weak (or weak ${ }^{*}$ ) topology and a $\sigma$-weakly closed ${ }^{*}$-subalgebra of some $\mathcal{B}(\mathcal{H})$ is a von Neumann algebra $\mathcal{M}$. This topological requirement can also be expressed in a purely algebraic way. For this, we introduce the commutant $X^{\prime}$ of some subset $X \subset \mathcal{B}(\mathcal{H})$, and define it to be the set of operators on $\mathcal{H}$ which commute with $X$,

$$
X^{\prime}=\{a \in \mathcal{B}(\mathcal{H}) \mid a x=x a \forall x \in X\} .
$$

The bi-commutant theorem of von Neumann asserts us that a $*$-subalgebra $\mathcal{M}$ of $\mathcal{B}(\mathcal{H})$ is weak-*-closed (and hence a von Neumann algebra) if and only if it is equal to the commutant of its commutant (its bi-commutant), $\mathcal{M}=\mathcal{M}^{\prime \prime}=\left(\mathcal{M}^{\prime}\right)^{\prime}$. States in the Hilbert space sense, i.e. density operators $\rho$, now induce normalized, positive, normal (i.e. $\sigma$-weakly continuous) linear functionals $\omega_{\rho}: \mathcal{M} \rightarrow \mathbb{C}$ via

$$
\omega_{\rho}(a)=\operatorname{Tr}(\rho a)
$$

for $a \in \mathcal{M}$. We note, however, that since $\mathcal{M}$ is in general not equal to all of $\mathcal{B}(\mathcal{H})$, the expectation values given by the above equation do not fix the density operator in a unique way. That is, in general there exists an equivalence class of density operators on $\mathcal{H}$, all corresponding to the same "physical" state. Hence the basic idea of the algebraic approach to quantum theory, is to think of the von Neumann algebra $\mathcal{M}$ as constructed above, as the fundamental object. States of the system are then described by linear, positive, normal, normalized functionals (i.e. maps to the complex numbers) on $\mathcal{M}$. By the definition of the weak-*-topology, every element of $\mathcal{M}$ defines a continuous linear functional on the Banach space $\mathcal{M}_{*}$ of linear and $\sigma$-weakly continuous functionals. It follows that $\mathcal{M}$ is the dual Banach space of $\mathcal{M}_{*},\left(\mathcal{M}_{*}\right)^{*}=\mathcal{M}$. Hence $\mathcal{M}_{*}$ is also called the pre-dual of $\mathcal{M}$. This duality property also allows from an abstract definition of a von Neumann algebra as a $\mathrm{C}^{*}$-algebra which is also a dual Banach space. These abstract objects are also referred to as $\mathrm{W}^{*}$-algebras.

Physical transformations. A measurement with outcomes parametrized by the index set $\Omega$ is given by a collection of positive elements $\left\{E_{\alpha}\right\}(\alpha$ in $\Omega$ ) of $\mathcal{M}$ (being an element of $\mathcal{M}$ and a positive operator on $\mathcal{H}$ ), summing up to the identity to preserve the normalization.

\footnotetext{
${ }^{2} \mathrm{~A}$ subalgebra closed under the action of the canonical involution (adjoint), in the physical community often abbreviated by $\dagger$
} 
Each element of this collection characterizes the probability $p(\alpha)$ of some outcome $\alpha$ of the measurement applied to the state $\omega$ via the formula

$$
p(\alpha)=\omega\left(E_{\alpha}\right) .
$$

This can be argued by assuming that the (un-normalized) state after measuring outcome $\alpha$ is given by $\omega_{\alpha}(x)=\omega\left(E_{\alpha}^{\frac{1}{2}} x E_{\alpha}^{\frac{1}{2}}\right)$, for $x \in \mathcal{M}$. The probability that outcome $\alpha$ is indeed detected is the evaluation with respect to the identity. Measurements are one example of physical transformations which are in general maps on $\mathcal{M}_{*}$ or between pre-duals of different von Neumann algebras $\mathcal{M}$ and $\mathcal{N}$, mapping states onto states. Hence the corresponding dual operation maps $\mathcal{N}$ into $\mathcal{M}$, and takes positive elements into positive elements. Moreover, it preserves the unit. It turns out that these requirements are not sufficient, since they are ill-behaved under certain extensions of the corresponding physical system.

Consider for example a quantum system $\mathcal{Q}$ described by a finite number of degrees of freedom, such as an atom in a cavity. Assuming that there are no further constraints on $\mathcal{Q}$, its observable algebra is equal to some finite-dimensional matrix algebra $M_{n}$, where $n$ is the number of degrees of freedom. If we now let $\mathcal{Q}$ interact with a quantum system modeled by a von Neumann algebra $\mathcal{M}$, then the overall observable algebra is given by the tensor product $M_{n} \otimes \mathcal{M}$. We note that the resulting von Neumann algebra is uniquely defined by the canonical inclusion

$$
M_{n} \otimes \mathcal{M} \subset M_{n} \otimes \mathcal{B}(\mathcal{H})=\mathcal{B}\left(\mathbb{C}^{n} \otimes \mathcal{H}\right)
$$

A physical transformation $T: \mathcal{N} \rightarrow \mathcal{M}$, mapping the von Neumann algebra $\mathcal{N}$ into $\mathcal{M}$, has to be positive with respect to all such extensions. That is we require that the map

$$
\operatorname{id}_{n} \otimes T: M_{n} \otimes \mathcal{N} \rightarrow M_{n} \otimes \mathcal{M}
$$

is positive for every $n \in \mathbb{N}$. Here, $\mathrm{id}_{n}$ is the identity map on the matrix algebra $M_{n}$. Such maps $T$ are called completely positive. A particular class of completely positive maps is given by finite collections $a_{i}$ of elements of $\mathcal{M}$, defining a map by

$$
T: \mathcal{M} \rightarrow \mathcal{M}, \quad T(x)=\sum_{i} a_{i}^{*} x a_{i} .
$$

A corresponding physical example would be a measurement where the outcomes are modeled by the positive elements $E_{i}$, and we take $a_{i}=E_{i}^{\frac{1}{2}}$. Then the map $T$ from above is the dual of the mapping describing the change of the physical state once the measurement is applied, forgetting the particular outcome. We refer the reader to the books of Takesaki [83] and Paulsen [70] for further details and structure theorems on completely positive maps.

The representation independent viewpoint. Instead of choosing the weak-*-topology, we could also choose the norm topology to complete a ${ }^{*}$-subalgebra of $\mathcal{B}(\mathcal{H})$. This would then lead us to the definition of a $C^{*}$-algebra, which is a more general object than a von Neumann algebra. In particular, one often defines $C^{*}$-algebras independent of the particular representation, i.e. by just requiring certain invariance properties under group actions, or by algebraic relations. A general physical system consisting of elementary particles (such as bosons or fermions) is characterized by a set of algebraic relations expressed through some 
set of generators $a_{i}$, indexed by some index set $i \in \mathcal{I}$. Algebraic relations are then given by polynomial equations with values in the set of generators. For example, we can choose the index set $\mathcal{I}$ to be the single particle Hilbert space $\mathcal{K}$ of a particle, and the algebraic relations as

$$
\begin{array}{r}
a_{f} a_{g}+a_{g} a_{f}=0, \quad f, g \in \mathcal{K} \\
a_{f} a_{g}^{*}+a_{g}^{*} a_{f}=\langle f, g\rangle_{\mathcal{K}} \mathbb{I}
\end{array}
$$

where $\langle., .\rangle_{\mathcal{K}}$ denotes the scalar product on $\mathcal{K}$. If moreover the association $\mathcal{K} \ni f \rightarrow a_{f}$ is anti-linear, then the physical system describes an infinite number of fermionic particles, each described by the single particle Hilbert space $\mathcal{K}$. In the case of spin- $\frac{1}{2}$ particles moving on a two-dimensional lattice, $\mathcal{K}$ would be equal to $\ell_{2}\left(\mathbb{Z}^{2}\right) \otimes \mathbb{C}^{2}$. Turning again to the more abstract situation, given such a set of generators $a_{i}$ and algebraic relations between them, we can form the $*$-algebra ${ }^{3} \mathcal{F}$ generated by them, that is the algebra generated by taking arbitrary sums and products as well as involutions of generators. This "free" *-algebra can be equipped with a norm by setting

$$
\|x\|=\sup _{\pi: \mathcal{F} \rightarrow \mathcal{B}(\mathcal{H})}\|\pi(x)\|_{\mathcal{B}(\mathcal{H})},
$$

where the supremum runs over all $*$-homomorphisms of $\mathcal{F}$ into the bounded operators $\mathcal{B}(\mathcal{H})$, and over all Hilbert spaces $\mathcal{H}$. It is easy to see the relation

$$
\left\|x^{*} x\right\|=\|x\|^{2}
$$

since it is in particular satisfied for every representation $\pi$. Moreover, we have that $\|a b\| \leq$ $\|a\|\|b\|$. These are exactly the defining properties of an abstract $\mathrm{C}^{*}$-algebra. It is easily checked that every norm closed subalgebra of some $\mathcal{B}(\mathcal{H})$ satisfies these requirements. The representation theorem of Gelfand, Naimark and Segal (GNS) tells us that in turn for every abstract $\mathrm{C}^{*}$-algebra $\mathcal{A}$ there exists a Hilbert space $\mathcal{H}$ and an injective (faithful) norm continuous $*$-homomorphism $\pi: \mathcal{A} \rightarrow \mathcal{B}(\mathcal{H})$.

Using this embedding, the definitions of positive elements of $\mathcal{A}$ and complete positivity of maps between $\mathrm{C}^{*}$-algebras are analogous to the case of von Neumann algebras. It is easy to see that an element $a \in \mathcal{A}$ is positive if there exists an element $x \in \mathcal{A}$ such that $a=x^{*} x$. Hence, a physical state on a $\mathrm{C}^{*}$-algebra $\mathcal{A}$ is a norm continuous map from $\mathcal{A}$ into the complex numbers, with the additional requirements that $\omega\left(x^{*} x\right) \geq 0$ and $\omega\left(\mathbb{I}_{\mathcal{A}}\right)=1$. Here, $\mathbb{I}_{\mathcal{A}}$ denotes the unit element (identity) of the $\mathrm{C}^{*}$-algebra $\mathcal{A}$, i.e. the element with the property $a \mathbb{I}_{\mathcal{A}}=\mathbb{I}_{\mathcal{A}} a=a$, for all $a \in \mathcal{A}^{4}$. It is easy to check that the map

$$
\langle., .\rangle_{\omega}: \mathcal{A} \times \mathcal{A} \rightarrow \mathbb{C}, \quad a \times b \mapsto\langle a, b\rangle_{\omega}=\omega\left(a^{*} b\right)
$$

${ }^{3} \mathrm{~A} *$-algebra is an algebra $\mathcal{F}$ such there exists an involution $*: \mathcal{F} \rightarrow \mathcal{F}$ fulfilling

$$
\begin{aligned}
\left(a^{*}\right)^{*}=a & (a b)^{*}=b^{*} a^{*} \\
(z a+y b)^{*}=\bar{z} a^{*}+\bar{y} b^{*} & \left\|a^{*}\right\|=\|a\| .
\end{aligned}
$$

\footnotetext{
${ }^{4}$ We note that not every $\mathrm{C}^{*}$-algebra possesses a unit, however, this obstacle can be removed by the process of unitization, see [83]. In the following, we always assume that a $\mathrm{C}^{*}$-algebra is unital, i.e. possesses a unit element.
} 
defines a sesquilinear form on the vector space $\mathcal{A}$ that is linear in the second variable and conjugate linear in the first. Together with the positivity requirement, we see that a state $\omega$ defines a pre-scalar product on the vector space $\mathcal{A}$, which can be turned into a Hilbert space after completion with respect to $\langle., .\rangle_{\omega}$ and taking the quotient with respect to the kernel of $\omega$. The corresponding Hilbert space is called the GNS Hilbert space $\mathcal{H}_{\omega}$ with respect to the state $\omega$. According to the construction, the elements of $\mathcal{H}_{\omega}$ can be approximately identified with elements of $\mathcal{A}$. We let $\xi_{\omega}$ be the vector corresponding to the unit element of $\mathcal{A}$. The multiplication from the left defines a $*$-homomorphism

$$
\pi_{\omega}: \mathcal{A} \rightarrow \mathcal{B}\left(\mathcal{H}_{\omega}\right) \quad \pi_{\omega}(a) b \xi_{\omega}=(a b) \xi_{\omega} .
$$

See any book on $\mathrm{C}^{*}$-algebras for a proof that the elements $a \xi_{\omega}$ are dense in $\mathcal{H}_{\omega}$ (the vector $\xi_{\omega}$ is cyclic), and that the above formal definition indeed extends to a $*$-representation, a *-homomorphism mapping the elements of $\mathcal{A}$ to bounded operators on $\mathcal{H}_{\omega}$. This is the GNS construction due to Gelfand, Naimark and Segal.

Hence we conclude that for every state $\omega$ on some physical observable algebra $\mathcal{A}$, there is a Hilbert space $\mathcal{H}_{\omega}$, a vector $\xi_{\omega}$ and a $*$-representation $\pi_{\omega}: \mathcal{A} \rightarrow \mathcal{B}\left(\mathcal{H}_{\omega}\right)$ such that

$$
\omega(a)=\left\langle\xi_{\omega} \mid \pi_{\omega}(a) \xi_{\omega}\right\rangle .
$$

For every such representation, we can construct the corresponding von Neumann algebra $\mathcal{M}=\pi_{\omega}(\mathcal{A})^{\prime \prime}$ by taking the double commutant. This $*$-subalgebra of $\mathcal{B}\left(\mathcal{H}_{\omega}\right)$ is closed in the weak-*-topology, and hence includes all physical "limit" observables. The positive functionals $\sigma$ which can be written in the form corresponding to equation (2.1),

$$
\sigma(x)=\sum_{i}\left\langle\xi_{\omega} \mid a_{i}^{*} x a_{i} \xi_{\omega}\right\rangle
$$

with $x \in \mathcal{M}$, and a finite number of $a_{i} \in \mathcal{M}$ are easily seen to be normal ${ }^{5}$. If we require that $\sum_{i} a_{i}^{*} a_{i}=\mathbb{I}_{\mathcal{H}_{\omega}}$, then these functionals naturally correspond to states which can be obtained from $\omega$ by the action of physical observables, starting from the state $\omega$. Hence the choice of a physical state of the abstract physical system modeled by $\mathcal{A}$ corresponds to the choice of a Hilbert space $\mathcal{H}$ and a von Neumann algebra of observables $\mathcal{M}$ acting on $\mathcal{H}$. All probability distributions corresponding to experiments starting with the state $\omega$ are then described by the evaluation of expectation values of elements of $\mathcal{M}$ with respect to normal states of $\mathcal{M}$. Hence, one could say that $C^{*}$-algebras are the abstract objects defining the theory, whereas von Neumann algebras correspond to 'physical realizations' of that theory. In order to derive predictions we have to study the representations of $\mathrm{C}^{*}$ algebras of physical observables obeying the physical symmetry conditions. We note that not all states give rise to equivalent physical realizations, which was first noted by Haag [34] in the case of the observable algebra of canonical commutator relations for systems with an infinite number of degrees of freedom. For more details on this, and the difficulty in choosing the "right" physical representation, we refer the reader to the book of Haag, [35].

We note for later reference that the double dual (as a Banach space) $\mathcal{A}^{* *}$ of a $\mathrm{C}^{*}$-algebra $\mathcal{A}$ is again a $\mathrm{C}^{*}$-algebra, and in fact is also an abstract von Neumann algebra. Its pre-dual is precisely the dual space $\mathcal{A}^{*}$ of $\mathcal{A}$. By Kaplansky's density theorem, we have that $\mathcal{A}$ is weak-*-dense in its double dual $\mathcal{A}^{* *}$.

\footnotetext{
${ }^{5}$ We can also take an infinite sequence $a_{i}$, and demand that the corresponding map is weak-*-continuous.
} 
Joint systems. Given two physical systems described by $\mathrm{C}^{*}$-algebras $\mathcal{A}$ and $\mathcal{C}$, the observable algebra of the joint system contains all elements of the algebraic tensor product $\mathcal{A} \otimes \mathcal{C}$, i.e. finite sums of elementary tensors $a \otimes b, a \in \mathcal{A}, b \in \mathcal{C}$. The algebraic tensor product is, however, not a $\mathrm{C}^{*}$-algebra since we have not yet specified a norm. Given two $\mathrm{C}^{*}$-algebras $\mathcal{A}, \mathcal{C}$, there are two canonical ways to turn their algebraic tensor product $\mathcal{A} \otimes \mathcal{C}$ into a $\mathrm{C}^{*}$-algebra. Consider two ${ }^{*}$-representations $\pi_{1}: \mathcal{A} \rightarrow \mathcal{B}\left(\mathcal{H}_{1}\right), \pi_{2}: \mathcal{C} \rightarrow \mathcal{B}\left(\mathcal{H}_{2}\right)$, and define the norm of some tensor $x \in \mathcal{A} \otimes \mathcal{C}$ as $\left\|\pi_{2} \otimes \pi_{2}(x)\right\|_{\mathcal{B}\left(\mathcal{H}_{A} \otimes \mathcal{H}_{B}\right)}$. The supremum over all such pairs of representations is called the minimal $\mathrm{C}^{*}$-algebraic tensor norm and will be denoted by $\otimes_{\min }$. It can be proven that it is indeed the smallest possible tensor norm for $\mathrm{C}^{*}$-algebras [83]. We denote by $\mathcal{A} \otimes_{\min } \mathcal{C}$ the completion of $\mathcal{A} \otimes \mathcal{C}$ with respect to this norm. We note that if $\mathcal{A} \subset \mathcal{B}\left(\mathcal{H}_{1}\right)$ and $\mathcal{C} \subset \mathcal{B}\left(\mathcal{H}_{2}\right)$ are two faithful representations, then the minimal tensor product norm arises from the completion of the canonical inclusion

$$
\mathcal{A} \otimes \mathcal{C} \subset \mathcal{B}\left(\mathcal{H}_{1} \otimes \mathcal{H}_{2}\right) .
$$

It follows that if $\mathcal{M}$ and $\mathcal{N}$ are two other $\mathrm{C}^{*}$-algebras such that we have two injective *-homomorphism (embeddings) of $\mathcal{A} \subset \mathcal{M}$, resp. $\mathcal{C} \subset \mathcal{N}$, then

$$
\mathcal{A} \otimes_{\min } \mathcal{C} \subset \mathcal{M} \otimes_{\min } \mathcal{N}
$$

which means that the minimal tensor product is injective with respect to the category of $\mathrm{C}^{*}$-algebras. The minimal tensor product is the natural tensor product taken in physics, since it is usually assumed that both parties hold different Hilbert spaces and the joint space is described by their tensor product.

To construct the maximal tensor norm, we do not only consider pairs of *-representations $\pi_{1}, \pi_{2}$ into the bounded operators on a pair of different Hilbert spaces, but also all pairs of ${ }^{*}$-representations into a single $\mathcal{B}(\mathcal{H})$, with the restriction that the range of $\pi_{1}$ commutes with the range of $\pi_{2}$. The resulting $\mathrm{C}^{*}$-structure, obtained after completion of $\mathcal{A} \otimes \mathcal{C}$, is called the maximal $\mathrm{C}^{*}$-tensor product and will be denoted by $\mathcal{A} \otimes_{\max } \mathcal{C}$. It is the largest possible tensor norm for $\mathrm{C}^{*}$-algebras [83].

Physically, this choice can be motivated by the fact that commuting observables do not influence each other. That is, let $\mathcal{O}_{1}=\left\{A_{i}\right\}$ and $\mathcal{O}_{2}=\left\{B_{j}\right\}$ be two finite sets of positive operators on some Hilbert space $\mathcal{H}$, both summing up to the identity, describing two possible measurements on some state $\omega$. According to the earlier discussion, the state after the outcome $i$ of measurement $\mathcal{O}_{1}$ was detected is given as

$$
\omega_{1, i}(x)=\omega\left(A_{i}^{\frac{1}{2}} x A_{i}^{\frac{1}{2}}\right) .
$$

Hence the probability of first detecting outcome $i$ of measurement $\mathcal{O}_{1}$ and then outcome $j$ of measurement $\mathcal{O}_{2}$ is equal to $p(i, j)=\omega\left(A_{i}^{\frac{1}{2}} B_{j} A_{i}^{\frac{1}{2}}\right)$. If every element of $\mathcal{O}_{1}$ commutes with every element of $\mathcal{O}_{2}$, then we get that this joint probability distributions is independent of the (time) ordering in which the measurements are performed, implying that the measurements do not influence each other.

Note that if either $\mathcal{A}$ or $\mathcal{C}$ is finite dimensional, then the two tensor norms induce the same $\mathrm{C}^{*}$-algebraic structure. This is also the defining property of nuclear $\mathrm{C}^{*}$-algebras [85, 74]. We note that all finite-dimensional matrix algebras are nuclear, since if $\mathcal{A} \subset \mathcal{B}(\mathcal{H})$ is an embedding, then the tensor product $M_{n} \otimes \mathcal{A}$ is uniquely defined by the inclusion

$$
M_{n} \otimes \mathcal{A} \subset \mathcal{B}\left(\mathbb{C}^{n} \otimes \mathcal{H}\right)
$$


We note that there are more examples for nuclear $\mathrm{C}^{*}$-algebras occurring in physics, see the discussion at the end of chapter 4 for details. A particular example is the observable algebra corresponding to canonical anti-commutation relations. In fact, it can be written as the infinite tensor product of qubit algebras ( $M_{2}$ matrix algebras). That is, it can be interpreted as the physical situation where the number of qubits is unlimited, but each observable approximable acts on a finite number of qubits. The corresponding von Neumann algebra also admits an interpretation familiar to quantum information theorists. This is explained next.

Infinitely many maximally entangled states. A qubit is the basic quantity of quantum information - in analogy to classical information theory, where the standard unit of information is the bit. Formally, a qubit is a physical system with two internal degrees of freedom. Hence its observable algebra is $M_{2}$, the algebra of two-by-two complex valued matrices. The standard communication resource is then a channel between two parties which sends bits and does not change their value. Equivalently, it is a pair of perfectly correlated bits. The analog in quantum information theory is the maximally entangled state $|\phi\rangle$ on two qubits, equal to

$$
|\phi\rangle=\frac{1}{\sqrt{2}}(|00\rangle+|11\rangle),
$$

where $|i i\rangle=|i\rangle \otimes|i\rangle \in \mathbb{C}^{4}$, and $|0\rangle,|1\rangle$ denote the standard orthonormal bases in $\mathbb{C}^{2}$. Here and throughout this thesis, we use the Dirac notation, especially for finite-dimensional Hilbert spaces. Maximally entangled states are a standard "resource" in quantum information theory, and most often the operational tasks results in the creation of maximally entangled states, from which most standard protocols can then be carried out. See [67] for more details on this. In particular, they can be used to quantify the amount of "entanglement" present in some physical state, [75]. In order to simplify the language, we follow the common speech in information theory and call two distinct parties simply Alice and Bob.

Let Alice and Bob denote two distinct parties, both holding one part of the maximally entangled state $|\phi\rangle$, which means that each of their observables look like $a \otimes \mathbb{I}_{\mathbb{C}^{2}}$. An unlimited amount of entanglement then corresponds formally to the infinite tensor product

$$
\bigotimes_{\mathbb{N}}|\phi\rangle .
$$

Consider now the set of vectors that can be obtained from this resource by the joint action of Alice and Bob, with the restriction that only an approximately finite number of maximally entangled states can be accessed at a time. This corresponds to the set of sequences $\left(|\psi\rangle_{n}\right)_{n \in \mathbb{N}}$, where $|\psi\rangle_{n} \in \mathbb{C}^{4}$ and we require

$$
\sum_{n} \||\psi\rangle_{n}-|\phi\rangle \|<\infty .
$$

Identifying $\left(|\psi\rangle_{n}\right)_{n \in \mathbb{N}}$ with the (infinite) formal tensor product $\bigotimes_{n \in \mathbb{N}}|\psi\rangle_{n}$, defining the canonical vector operations, we see that the resulting vector space can be equipped with the scalar product

$$
\left\langle\bigotimes_{n \in \mathbb{N}} \psi_{n} \mid \bigotimes_{n \in \mathbb{N}} \mu_{n}\right\rangle=\prod_{n \in \mathbb{N}}\left\langle\psi_{n} \mid \mu_{n}\right\rangle_{\mathbb{C}^{4}}
$$


resulting in the Hilbert space $\mathcal{H}_{e}$ of an unlimited amount of bipartite entanglement. The observable algebra corresponding to local actions on Alice's side involving only finitely many copies of one part of maximally entangled states is then given by

$$
\tilde{\mathcal{R}}=\bigcup_{X \subset \mathbb{N},|X|<\infty} \bigotimes_{x \in X} M_{2}
$$

where $|X|$ is the number of elements in the set $X$. We note that it is not yet a von Neumann algebra, since it is not closed in the weak-*-topology. Consider its action on the Hilbert space constructed above, defined by the $*$-homomorphism

$$
\pi\left(\bigotimes_{k} x_{k}\right) \bigotimes_{\mathbb{N}}|\phi\rangle=\bigotimes_{\mathbb{N}} x_{k} \otimes \mathbb{1}_{\mathbb{C}^{2}}|\phi\rangle
$$

It is easily checked that this action generates the Hilbert space $\mathcal{H}_{e}$, which implies that $\bigotimes_{\mathbb{N}}|\phi\rangle$ is a cyclic vector. Moreover, we see that the state $\tau$ associated to the vector $\bigotimes_{\mathbb{N}}|\phi\rangle$ is tracial, i.e. we have that

$$
\tau(a b)=\tau(b a)
$$

for elements $a, b \in \tilde{\mathcal{R}}$ and hence also for all sequences with elements in $\tilde{\mathcal{R}}$ which converge with respect to the weak topology induced by the semi-norms $\sigma_{a}(x)=\tau(a x)$. Since $\bigotimes_{\mathbb{N}}|\phi\rangle$ is cyclic, this is just the weak operator topology on the Hilbert space $\mathcal{H}_{e}$. Thus this closure $\mathcal{R}$ of the $*$-subalgebra $\tilde{\mathcal{R}}$ of $\mathcal{B}\left(\mathcal{H}_{e}\right)$, or equivalently the double commutant, is then the von Neumann algebra describing the local action of Alice. It was first considered by von Neumann himself and is called the unique hyperfinite type II $I_{1}$ factor. The construction above is due to Araki and Woods, [3].

Generally, a von Neumann algebra $\mathcal{M}$ is called hyperfinite, if it can be approximated (in the weak-*-topology) by a net of matrix algebras of increasing dimension. It is called a factor, if the intersection with its commutant only contains multiples of the identity operator,

$$
\mathcal{M} \cap \mathcal{M}^{\prime}=\{z \mathbb{I}\}
$$

with $z \in \mathbb{C}$. An infinite-dimensional von Neumann algebra which is a factor and admits a tracial state is named a type $I I_{1}$ factor. Due to the earlier work of Murray and von Neumann [64], there exists only one (up to isomorphisms) hyperfinite type $I I_{1}$ factor, the one just constructed. The property of hyperfiniteness is closely related to injectivity and both properties are equivalent for von Neumann algebras on separable Hilbert spaces. A von Neumann algebra $\mathcal{N}$ is called injective, if for any $\mathrm{C}^{*}$-algebra $\mathcal{A}$ and any selfadjoint subspace $S \subset \mathcal{A}$ containing the identity, every completely positive map $\varphi: S \rightarrow \mathcal{N}$ can be extended to a map $\tilde{\varphi}: \mathcal{A} \rightarrow \mathcal{N}$. A deep result of Arveson [4], see also [70], states that the von Neumann algebra of bounded operators $\mathcal{B}(\mathcal{H})$ is injective. This was used by Loebl, [58], to show that a von Neumann algebra $\mathcal{N} \subset \mathcal{B}(\mathcal{H})$ is injective, if and only if there exists a completely positive unital projection $P: \mathcal{B}(\mathcal{H}) \rightarrow \mathcal{N}$, i.e. satisfying $\left.P\right|_{\mathcal{N}}=\mathrm{id}_{\mathcal{N}}$. Such a map is also called a conditional expectation. One direction can be seen from the fact that since $\mathcal{B}(\mathcal{H})$ is injective, the inclusion map extends to a projection. For the converse, note that if $\varphi: S \rightarrow \mathcal{N} \subset \mathcal{B}(\mathcal{H})$ is as above, then there exists an extension $\tilde{\varphi}: \mathcal{A} \rightarrow \mathcal{B}(\mathcal{H})$, and the composition $P \circ \tilde{\varphi}: \mathcal{A} \rightarrow \mathcal{N}$ is as desired. The equivalence of hyperfiniteness and injectivity is due to the work of Connes, proven in the same paper were he stated his embedding conjecture. 
The hyperfinite type $I I_{1}$ factor describes the physical situation of two parties holding an unlimited amount of maximally entangled qubit pairs and each acting on (approximately) finitely many of them. Since maximal entangled qubit pairs are the standard resource in quantum information theory, it is natural to ask whether this is a "typical" situation, that is, what kind of operational tasks can be understood in this way. In information theory one usually asks for asymptotic equivalence of tasks, i.e. equivalence in the limit of infinitely many runs of the protocol. Hence the corresponding local operations on Alice' side are sequences

$$
\left(a_{i}\right)_{i \in \mathbb{N}}
$$

where for each run $i \in \mathbb{N}$ of the protocol $a_{i}$ is a local operation on one part of (approximately) finitely many maximally entangled qubits, which implies $a_{i} \in \mathcal{R}$. It is then natural to ask for the limit of this sequence in $\mathcal{R}$. Since $\mathcal{R}$ is weakly closed, we see that in order for it to exist, the sequence has to be a Cauchy sequence with respect to the weak-*-topology. Due to the construction of $\mathcal{R}$ this implies that for large $n$, Alice's operations have to be almost the same, i.e. there is a number $N \in \mathbb{N}$ such that for all $n, m>N$ the operators $a_{n}$ and $a_{m}$ are arbitrarily close with respect to their actions on maximally entangled pairs. However, we did not require that individual runs of the protocol have to be the same. By approximating every element of the sequence $\left(a_{i}\right)_{i \in \mathbb{N}}$ by operations on exactly only finitely many qubits, we see that we are interested in convergent subsequences. Hence a broader definition of convergence is needed. For that, we need a little excursion into topology and the notion of ultrafilters.

Ultrafilters and ultraproducts. We start with the definition of an ultrafilter on some index set, denoted by $\mathcal{I}$. In general, we do not specify the index set any further, but the reader may think of it as the set of natural numbers $\mathbb{N}$. An ultrafilter on some index set $\mathcal{I}$ is a set $\mathcal{U}$ of subsets of $\mathcal{I}$ such that

(i) the empty set is not an element of $\mathcal{U}$,

(ii) if $A \subset B \subset \mathcal{I}$ and $A \in \mathcal{U}$ then $B \in \mathcal{U}$,

(iii) if $A, B \in \mathcal{U}$ then their intersection $A \cap B$ is also an element of $\mathcal{U}$,

(iv) if $A \subset \mathcal{I}$ then either $A \in \mathcal{U}$ or $\mathcal{I} \backslash A \in \mathcal{U}$ is a true statement.

If an ultrafilter $\mathcal{U}$ possesses a least element, it is called principal, and if not, then $\mathcal{U}$ is called a free ultrafilter. The existence of ultrafilters follows from Zorn's lemma, which implies that every filter $\mathcal{F}$ (on $\mathcal{I}$ ) is dominated by some ultrafilter $\mathcal{U}$ (every element of $\mathcal{F}$ is also an element of $\mathcal{U}$ ). Like filters, ultrafilters allow for a generalization of convergence. We let $\mathcal{U}$ be an ultrafilter on $\mathcal{I}$, and let $\left(x_{\alpha}\right)_{\alpha \in \mathcal{I}}$ be a sequence of elements of some metric space $(X, d)$, indexed by $\mathcal{I}$. The sequence is called convergent along $\mathcal{U}$ with limit point $x$, denoted by $\lim _{\mathcal{U}} x_{\alpha}=x$, if we have that for every $\varepsilon>0$

$$
\left\{\alpha \in \mathcal{I}: d\left(x_{\alpha}, x\right)<\varepsilon\right\} \in \mathcal{U} .
$$

With this definition at hand, it is for example easy to see that every sequence indexed by the natural numbers and with values in some compact Hausdorff space converges along any ultrafilter on $\mathbb{N}$. It also follows that the limit $x$ lies in the set of clusterpoints of $x_{\alpha}$, i.e. 
there is a subsequence converging to $x$. The converse direction is also true. We refer the reader to a book on general topology for the proofs of these statements.

The notion of ultrafilters now allows for a reconsideration of sequences $\left(a_{i}\right)_{i \in \mathbb{N}}$ with elements in $\mathcal{R}$. Since we are interested in physical actions, we have to take positive elements in $\mathcal{R}$, i.e. sequences of the form $\left(a_{n}^{*} a_{n}\right)$. We see that for any such sequence, the complex valued sequence $\tau\left(a_{n}^{*} a_{n}\right)$ converges along a free ultrafilter $\mathcal{U}$ on the set of natural numbers. With respect to that ultrafilter, all sequences which differ up to a sequence $\left(b_{n}\right)$ with $\lim _{\mathcal{U}} \tau\left(b_{n}^{*} b_{n}\right)=0$ are indistinguishable. Hence the object of interest is the quotient

$$
(\mathcal{R})_{\mathcal{U}} \equiv \prod_{n \in \mathbb{N}} \mathcal{R} / \mathcal{K}_{\mathcal{U}}
$$

where $\mathcal{K}_{\mathcal{U}}=\left\{\left(b_{n}\right) \mid b_{n} \in \mathcal{R}, \lim _{\mathcal{U}} \tau\left(b_{n}^{*} b_{n}\right)=0\right\}$ is a two-sided ideal. These objects were first considered by McDuff, [62], who proved that for any free ultrafilter $\mathcal{U}$ on the natural numbers, $(\mathcal{R})_{\mathcal{U}}$ is a von Neumann algebra of type $I I_{1}$ with the tracial state being

$$
\tau_{\mathcal{U}}(x)=\lim _{\mathcal{U}} \tau\left(x_{n}\right),
$$

where $\left(x_{n}\right)$ is any representing sequence of $x \in(\mathcal{R})_{\mathcal{U}}$. We call $(\mathcal{R})_{\mathcal{U}}$ the ultrapower of the hyperfinite factor $\mathcal{R}$ with respect to a free ultrafilter on the natural numbers. We note that the question as to whether all ultrapowers of $\mathcal{R}$ are isomorphic is equivalent to the continuum hypothesis, [31, 25]. From our considerations of quantum information protocols it follows that the subalgebras of $(\mathcal{R})_{\mathcal{U}}$ are of particular interest. This leads to the embedding problem of Alain Connes.

\subsection{The conjectures of Connes and Kirchberg}

The embedding conjecture of Alain Connes. In his seminal paper "Classification of injective factors. Cases $I I_{1}, I I_{\infty}, I I I_{\lambda}, \lambda \neq 1$ " [17], which appeared in the year 1976, Alain Connes answered a number of open problems in the classification of von Neumann algebras. In particular, he proved that all injective factors of type $I I_{1}$ acting on a separable Hilbert space are isomorphic to the unique hyperfinite factor $\mathcal{R}$ of the same type. In passing, he also studied tracial ultrapowers of this factor and gave a necessary condition for a finite ${ }^{6}$ factor to be embeddable into the ultrapower of the hyperfinite factor. We quote from his paper:

We now construct an approximate imbedding of [a finite factor] $\mathcal{N}$ in $R$. Apparently such an imbedding ought to exist for all $I I_{1}$ factors because it does for the regular representation of free groups. However, the construction [..] relies on the condition [which we state now].

In the following, $\operatorname{Tr}$ is the usual (unnormalized) trace on $\mathcal{H}$, so that if $p$ is a projection onto a finite-dimensional subspace $p \mathcal{H}$ of $\mathcal{H}$, the dimension of $p \mathcal{H}$ is given by $\operatorname{Tr} p$. We denote by $\|.\|_{H S}$ and $\langle. \mid .\rangle_{H S}$ the Hilbert-Schmidt norm and scalar product for operators on $\mathcal{H},\langle a \mid b\rangle_{H S}=\operatorname{Tr} a^{*} b,\|a\|_{H S}^{2}=\langle a \mid a\rangle_{H S}$.

\footnotetext{
${ }^{6}$ If $\mathcal{N}$ is a finite-dimensional von Neumann algebra, then it trivially embeds into the hyperfinite factor and hence into its ultrapower.
} 
Theorem 2.2.1 (A.Connes, [17]). Let $\mathcal{N}$ be a factor of type $I I_{1}$ acting in standard form on some Hilbert space $\mathcal{H}$, and let $\tau$ be the normalized trace on $\mathcal{N}$. Assume that for every finite collection $x_{1}, \ldots, x_{n}$ of elements of $\mathcal{N}$ and any $\varepsilon>0$ there exist a non-zero finitedimensional projection $p \in \mathcal{B}(\mathcal{H})$ such that for all $j \in\{1, \ldots, n\}$ we have

$$
\left\|\left[x_{j}, p\right]\right\|_{H S} \leq \varepsilon\|p\|_{H S}, \quad\left|\tau\left(x_{j}\right)-\frac{1}{\langle p \mid p\rangle_{H S}}\left\langle x_{j} p \mid p\right\rangle_{H S}\right| \leq \varepsilon .
$$

Then there exists a free ultrafilter on the natural numbers $\mathbb{N}$ and a normal homomorphism of $\mathcal{N}$ into the ultrapower $(\mathcal{R})_{\mathcal{U}}$ of the hyperfinite $I I_{1}$ factor.

Whether this condition is really necessary or whether such an embedding exists for any type $I I_{1}$ factor is called "Connes' embedding problem". We see already that it is inherently connected to the existence of finite-dimensional approximations of $\mathcal{N}$. In fact, Connes used the existence of finite-dimensional projections $p$ fulfilling (2.2) to construct finite-dimensional approximations for the unitary operators in $\mathcal{N}$ approximating the tracial values of the original unitaries. That these finite-dimensional approximations of unitary elements are crucial became much clearer with the seminal work "On non-semisplit extensions, tensor products and exactness of group C*-algebras" by Eberhard Kirchberg. Among other equivalences, which we discuss later on, he obtained the following characterization of finite factors which embed into the ultrapower $(\mathcal{R})_{\mathcal{U}}$.

Theorem 2.2.2 (E.Kirchberg, [55]). Let $\mathcal{N}$ be a von Neumann algebra with separable predual and a normal and faithful trace $\tau$. Then there exists a free ultrafilter $\mathcal{U}$ and a normal homomorphism $h: N \rightarrow(\mathcal{R})_{\mathcal{U}}$ with $\tau_{\mathcal{U}}(h(x))=\tau(x), x \in N$, if and only if for every $\varepsilon>0$ and every finite sequence $\left\{u_{1}, \ldots, u_{n}\right\}$ of unitary elements in $\mathcal{N}$, there exist a natural number $k$ and unitary matrices $\left\{v_{1}, \ldots, v_{n}\right\}$ in the matrix algebra $M_{k}$ such that

$$
\left|\tau\left(u_{i}^{*} u_{j}\right)-\frac{1}{k} \operatorname{Tr}\left(v_{i}^{*} v_{j}\right)\right| \leq \varepsilon,
$$

for $i, j \in\{1, \ldots, n\}$.

Actually, he also required that $\left|\tau\left(u_{i}\right)-\frac{1}{k} \operatorname{Tr}\left(v_{i}\right)\right| \leq \varepsilon$ holds, but as Dykema and Juschenko noticed [21], this formally stronger condition is easily satisfied by adjoining the identity $\mathbb{I}_{\mathcal{N}}$ to the set of unitaries.

The asymptotic quantum Birkhoff property. The characterization of Kirchberg also leads to a direct connection to quantum information theory. This was first discovered by Haagerup and Musat [37] during their work on $\tau_{n}$-factorizable maps on matrix algebras. A completely positive unital map $T: M_{n} \rightarrow M_{n}$ is called trace preserving, if $\operatorname{Tr} T(x)=\operatorname{Tr} x$. Such a map $T$ is called factorizable, if there exists a finite von Neumann algebra $\mathcal{N}$ with a faithful normal tracial state $\tau$, and a unitary operator $u$ in the von Neumann algebra $M_{n} \otimes \mathcal{N}$ such that

$$
T(x)=\operatorname{id}_{n} \otimes \tau\left(u^{*} x \otimes \mathbb{I}_{\mathcal{N}} u\right) .
$$

Factorizable maps were introduced by Anantharaman-Delaroche [2], albeit defining them in a different way. The equivalence to the above definition is due to Haagerup and Musat [37]. They also noted the connection to the asymptotic quantum Birkhoff property, introduced 
by Verstraete, Smolin and Winter in [82]. A completely positive unital and trace preserving map $T: M_{n} \rightarrow M_{n}$ is said to have the asymptotic quantum Birkhoff property, if we have

$$
\lim _{k \rightarrow \infty} d_{c b}\left(\bigotimes_{i=1}^{k} T, \operatorname{conv}\left(\operatorname{Aut}\left[M_{n^{k}}\right]\right)\right)=0,
$$

where Aut $\left[M_{n^{k}}\right]$ denotes the automorphism group of the matrix algebra $M_{n^{k}}$, and $d_{c b}$ is the metric corresponding to the completely bounded norm. Hence we ask for whether it is possible to write tensor products of $T$ approximately as convex combinations of terms like $u^{*}$.u, for unitary matrices $u \in M_{n^{k}}$. The question whether all completely positive unital and trace preserving map $T: M_{n} \rightarrow M_{n}$ have the asymptotic quantum Birkhoff property, known as the asymptotic quantum Birkhoff conjecture, was solved in the negative by Haagerup and Musat, by showing that the asymptotic quantum Birkhoff property implies factorizability, and showing the existence of non-factorizable maps. The converse would imply Connes embedding problem.

Theorem 2.2.3 (Haagerup and Musat, [37]). Let $n \geq 3$ and consider a completely positive unital and trace-preserving map $T: M_{n} \rightarrow M_{n}$ which is factorizable. If all such maps have the asymptotic quantum Birkhoff property, then Connes' embedding problem has a positive answer.

See Remark 6.3 in [37] for information on the converse statement. As already mentioned, their proof uses the characterization of Kirchberg. Hence it is worthwhile to study the set of matrices of the form

$$
c_{i j}=\tau\left(u_{i}^{*} u_{j}\right)_{1 \leq n}
$$

for some finite factor $\mathcal{N}$, unitary elements $\left\{u_{1}, \ldots, u_{n}\right\} \in \mathcal{N}$ and its trace $\tau$. These matrices are self-adjoint and their diagonal entries are all equal to one, and so form a subset of the set of correlation matrices, which is the set of all matrices obeying these two conditions. That the former set (2.4) is a true subset of the set of all correlation matrices (starting from $n>3$ ), was proven in [21]. This result was accomplished by studying the extreme points in the set of correlation matrices.

Algebraic formulations. The main achievement of the paper of Juschenko and Popovych [49] is a purely algebraic formulation of the Connes' embedding problem, based on Kirchberg's characterization. It starts with the following reformulation of Kirchberg's condition.

Corollary 2.2.4 (Juschenko and Popovych,[49]). Let $\mathcal{N}$ be a finite factor with separable predual, and let $m_{i j}, i, j \in\{1, \ldots, n\}$ be an $n \times n$ complex valued matrix with constant diagonal entries, $m_{i i}=m_{k k}=m, \forall i, k \in\{1, \ldots, n\}$. Moreover, assume that for every positive integer $d \in \mathbb{N}$ and all collections of $n$ unitary matrices $v_{1}, \ldots, v_{n}$ acting on a d-dimensional Hilbert space,

$$
m+\frac{1}{d} \sum_{i \neq j} m_{i j} \operatorname{Tr}\left(v_{i}^{*} v_{j}\right) \geq 0
$$

holds. If it follows that

$$
m+\frac{1}{d} \sum_{i \neq j} m_{i j} \tau\left(u_{i}^{*} u_{j}\right) \geq 0
$$


for every collection of $n$ unitary elements $u_{1}, \ldots, u_{n}$ in $\mathcal{N}$, and any such matrix $m$, then $\mathcal{N}$ can be embedded into $(R)_{\mathcal{U}}$.

Juschenko and Popovych [49] also considered the subset of real valued correlation matrices and noticed that each element of this set indeed admits a representation of the desired form. More precisely, any symmetric real valued $n \times n$ matrix $m$ with only ones on the diagonal can be represented using unitaries acting on a finite-dimensional Hilbert space. To see this, note that any real valued correlation matrix is a Gram matrix for a set of $n$ vectors $x_{i}$ in an $n$-dimensional real Hilbert space, and hence

$$
c_{i j}=\left\langle x_{i} \mid x_{j}\right\rangle=\sum_{k l} x_{i}^{k} x_{j}^{l} \frac{1}{2^{n}} \operatorname{Tr}\left(\gamma_{k} \gamma_{l}\right),
$$

where $\gamma_{k}$ are the self-adjoint unitary generators of the Clifford algebra of order $n^{7}$. Since the Clifford relations imply that $\sum_{k} x^{k} \gamma_{k}$ for some unit vector with entries $x^{k}$ is again self-adjoint and unitary, the result follows.

As explained above, for matrices $m_{i j}$ with constant diagonal and real valued entries, the conditions in equations (2.5) and (2.6) are in fact equivalent. The next step is to see that set of matrices $m_{i j}$ considered in the last corollary admits a purely algebraic description. For that, we need the notion of free groups.

The free group $\mathbb{F}_{n}$ with $n$ generators $g_{1}, \ldots, g_{n}$ is the group formed by all words written in a unique way as the product of the generators together with their inverses, using the only cancelation rule $g_{i} g_{i}^{-1}=e$; $e$ being the empty word. We denote by $\mathbb{F}_{\infty}$ the inductive limit of the sequence $\mathbb{F}_{n}$, the free group with countably many generators. Note that any group can be realized as a quotient of $\mathbb{F}_{\infty}$.

Every complex valued matrix $m_{i j}$ with constant diagonal corresponds to a second-order element $\tilde{m}$ of the free $*$-group algebra $\mathcal{F}$,

$$
\tilde{m}\left(u_{1}, \ldots, u_{n}\right)=m e+\sum_{i \neq j} m_{i j} u_{i}^{*} u_{j} .
$$

An element of $\mathcal{F}$ is said to be a sum of hermitian squares, if it is of the form $\sum_{i} g_{i}^{*} g_{i}$, for some collection $g_{i} \in \mathcal{F}$. A commutator in $\mathcal{F}$ is an element of the form $[f m-m f]$, $m, f \in \mathcal{F}$. Two elements $m_{1}, m_{2}$ in $\mathcal{F}$ are called cyclically equivalent, if $m_{1}=m_{2}+c$, where $c$ is a sum of commutators in $\mathcal{F}$. The algebraic formulation of Connes' embedding problem in terms of second-order elements of $\mathcal{F}$ now reads as follows.

Theorem 2.2.5 (Juschenko and Popovych,[49]). Connes' embedding problem is true if and only if for any second-order element $\tilde{m} \in \mathcal{F}$ given by some complex valued matrix $m_{i j}$ with constant diagonals, the condition of Corollary 2.2.4 implies that for any $\varepsilon>0, \varepsilon+m$ is cyclically equivalent to a sum of hermitian squares.

The proof is based on contradiction. The basic idea being that if the statement is not true, then there exists a functional separating $\tilde{m}$ from the set of elements which are cyclically

\footnotetext{
${ }^{7}$ The Clifford algebra of order $n$ is characterized by the relations$$
\gamma_{k} \gamma_{l}+\gamma_{l} \gamma_{k}=2 \delta_{k l}
$$

of its $n$ generators $\gamma_{k}$. It can be faithfully represented on a complex Hilbert of dimension $2^{n}$, for example by representing its generators by tensor products of Pauli matrices.
} 
equivalent to a sum of hermitian squares. This functional vanishes on commutators and hence can be extended to a tracial state on the universal enveloping $\mathrm{C}^{*}$-algebra $C^{*}(\mathcal{F})=$ $C^{*}\left(\mathbb{F}_{\infty}\right)$. The corresponding GNS representation generates a type $I I_{1}$ factor, which if embedded into the ultrapower $(R)_{\mathcal{U}}$ gives rise to sequence of finite-dimensional unitary matrices such that the trace of $\tilde{m}$ evaluated on these matrices is negative, contradicting the assumption. For details, we refer the reader to [49].

The work of [49] was inspired by some similar result of Klep and Schweighofer [56], who obtained the first purely algebraic reformulation of Connes' embedding problem. Instead of the free group and its associated algebra, they considered the vector space $\mathcal{K}\left\langle X_{1}, \ldots, X_{n}\right\rangle$ of polynomials in $n$ non-commuting variables $X_{1}, \ldots, X_{n}$ and complex coefficients. Then the definitions of hermitian squares, commutators and cyclical equivalence admit a canonical meaning on $\mathcal{K}\left\langle X_{1}, \ldots, X_{n}\right\rangle$. A quadratic module in $\mathcal{K}\left\langle X_{1}, \ldots, X_{n}\right\rangle$ is a subset which contains the unit and is closed under addition and the conjugation $f \rightarrow g^{*} f g$ for some element $g \in \mathcal{K}\left\langle X_{1}, \ldots, X_{n}\right\rangle$, where the involution is defined in the canonical way. An element $f \in \mathcal{K}\left\langle X_{1}, \ldots, X_{n}\right\rangle$ is called self-adjoint, if $f^{*}=f$. The result of Klep and Schweighofer [56] now reads as follows.

Theorem 2.2.6 (Klep and Schweighofer, [56]). Let $f \in \mathcal{K}\left\langle X_{1}, \ldots, X_{n}\right\rangle$ be a self-adjoint element. Then the following statements are equivalent if and only if Connes' embedding conjecture holds.

1. For all positive integers $d$ and any set of $n$ self-adjoint matrices $a_{1}, \ldots, a_{n}$ of dimension $d$ with norm smaller or equal to one,

$$
\operatorname{Tr}\left(f\left(a_{1}, \ldots, a_{n}\right)\right) \geq 0 .
$$

2. For any $\varepsilon>0$, the element $\varepsilon+f$ is cyclically equivalent to an element in the quadratic module generated by the elements of the form $1-X_{i}^{2}, i \in\{1, \ldots, n\}$.

The proof is again based on the construction of some tracial state on the universal enveloping $\mathrm{C}^{*}$-algebra of $\mathcal{K}\left\langle X_{1}, \ldots, X_{n}\right\rangle$. As already mentioned, there exists many other equivalent statements of Connes' embedding problem, especially with respect to groups and their unitary representations. A countable discrete group $G$ is called hyperlinear, if there exists an injective group homomorphism ("an embedding") of $G$ into the unitary group of the ultrapower $(\mathcal{R})_{\mathcal{U}}$. It follows that the Connes' embedding problem asks whether all countable discrete groups are hyperlinear. If so, then this would refute a famous "meta theorem" of Gromov, stating that a proposition which holds for all countable discrete groups is either trivial or false.

All these equivalences ask for the existence of finite-dimensional approximations for the elements of the unitary group of some von Neumann algebra $\mathcal{N}$. Since the fundamental work of Grothendieck, [33], it is known that the approximation properties of Banach spaces is inherently connected to the theory of their tensor products. That is, the existence of approximations for some Banach space $B$ can often be examined via the potential tensor norms on the algebraic tensor product $B \otimes C$, where $C$ varies in some set of Banach spaces. See the next chapter for a more detailed discussion with respect to the category of archimedian order unit vector spaces with involution. Hence it was natural to relate the approximation properties of $\mathrm{C}^{*}$ - and von Neumann algebras to their behavior under tensoring, a program put forward by Lance [57], Lance and Effros [23] and further developed by Effros and Choi [15]. 
Approximation properties of nuclear $\mathbf{C}^{*}$-algebras. Their prime result was that a nuclear $\mathrm{C}^{*}$-algebra $\mathcal{A}$ admits an approximation of the identity map factoring through matrix algebras. To be precise, there exists a net of pairs of completely positive unital maps

$$
u_{\varepsilon}: \mathcal{A} \rightarrow M_{n(\varepsilon)} \quad v_{\varepsilon}: M_{n(\varepsilon)} \rightarrow \mathcal{A}
$$

such that the following diagram approximately commutes with respect to the point-norm topology.

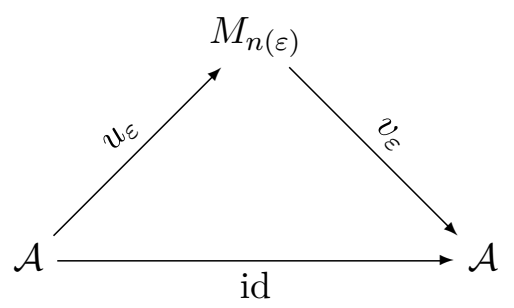

By approximately commuting, we mean that for every $\varepsilon>0$, there is an integer $n(\varepsilon)$ such that we have for the corresponding maps $\left\|v_{\varepsilon} \circ u_{\varepsilon}(a)-a\right\| \leq \varepsilon\|a\|$, for all $\varepsilon>0$ and $a$ in $\mathcal{A}$. A von Neumann algebra $\mathcal{N}$ admitting the same factorization property for its identity map, but with respect to the weak-*-topology, is called semi-discrete. Due to the work of Connes [17] (see Haagerup [36] for a simpler proof), we know that for factors on a separable Hilbert space the properties of hyperfiniteness, semi-discreteness and injectivity are all equivalent. Furthermore, we have that a separable $\mathrm{C}^{*}$-Algebra $\mathcal{A}$ is nuclear if and only if its double dual $\mathcal{A}^{* *}$ is injective. It follows that the property of nuclearity is quite strong.

The weak expectation property. The defining property of the minimal tensor product is that it is injective. In analogy, Lance [57] introduced the weak expectation property for $\mathrm{C}^{*}$-algebras, in short the WEP. A $\mathrm{C}^{*}$-algebra $\mathcal{A}$ has the WEP if for all faithful inclusions $\mathcal{A} \subset \mathcal{B}(\mathcal{H})$ and any other $\mathrm{C}^{*}$-algebra $\mathcal{B}$, the canonical $*$-homomorphism $\mathcal{A} \otimes \mathcal{B} \rightarrow \mathcal{B}(\mathcal{H}) \otimes \mathcal{B}$ is continuous with respect to the maximal tensor product, which implies the embedding

$$
\mathcal{A} \otimes_{\max } \mathcal{B} \subset \mathcal{B}(\mathcal{H}) \otimes_{\max } \mathcal{B}
$$

A particular choice for $\mathcal{B}$ is the commutant $\mathcal{A}^{\prime}$, computed with respect to the inclusion $\mathcal{A} \subset \mathcal{B}(\mathcal{H})$. By the definition of the maximal tensor product, the map

$$
\begin{aligned}
& \iota_{\mathcal{A}} \otimes \iota_{\mathcal{A}^{\prime}}: \mathcal{A} \otimes_{\max } \mathcal{A}^{\prime} \rightarrow \mathcal{B}(\mathcal{H}) \\
& \sum_{i} a_{i} \otimes b_{i} \mapsto \sum_{i} \iota_{\mathcal{A}}\left(a_{i}\right) \cdot \iota_{\mathcal{A}^{\prime}}\left(b_{i}\right)
\end{aligned}
$$

is completely positive, where $\iota_{\mathcal{A}}, \iota_{\mathcal{A}}$ denote the respective inclusion maps into $\mathcal{B}(\mathcal{H})$. Since $\mathcal{B}(\mathcal{H})$ is injective, using equation $(2.7)$ this map can be extended to a completely positive map $\varphi: \mathcal{B}(\mathcal{H}) \otimes_{\max } \mathcal{A}^{\prime} \rightarrow \mathcal{B}(\mathcal{H})$. Its restriction to $\mathcal{A}^{\prime}$ is furthermore a $*$-homomorphism. By Choi's theory of multiplicative domains, see chapter 3 of the book of Paulsen, [70], it follows that $\varphi$ is a $\mathcal{A}^{\prime}$-bimodule map,

$$
\varphi(a x)=a \varphi(x) \quad \forall a \in \mathcal{A}^{\prime}, x \in \mathcal{B}(\mathcal{H}) \otimes_{\max } \mathcal{A}^{\prime} .
$$

Hence we have for the restriction $\tilde{\varphi}(x)=\varphi(x \otimes \mathbb{I}), x \in \mathcal{B}(\mathcal{H})$

$$
a \tilde{\varphi}(x)=a \varphi(x \otimes \mathbb{I})=\varphi(x \otimes a)=\varphi(x \otimes \mathbb{I}) a=\tilde{\varphi}(x) a,
$$


which implies that $\tilde{\varphi}(x) \in \mathcal{A}^{\prime \prime}$ for $x \in \mathcal{B}(H)$. Furthermore, we have that $\tilde{\varphi}(x)=x$, for $x \in \mathcal{A}$. If we choose the Hilbert space $\mathcal{H}$ such that we have a faithful embedding $\mathcal{A} \subset \mathcal{A}^{* *} \subset$ $\mathcal{B}(\mathcal{H})$, we obtain from the above procedure a completely positive map $\tilde{\varphi}: \mathcal{B}(\mathcal{H}) \rightarrow \mathcal{A}^{* *}$, with $\left.\tilde{\varphi}\right|_{\mathcal{A}}=\mathrm{id}_{\mathcal{A}}$. This result is due to Lance. In general, if $\mathcal{B}$ and $\mathcal{A} \subset \mathcal{A}^{* *} \subset \mathcal{B}$ are $\mathrm{C}^{*}$-algebras, we call a completely positive map $\phi: \mathcal{B} \rightarrow \mathcal{A}^{* *}$ a weak expectation, if $\left.\phi\right|_{\mathcal{A}}=\mathrm{id}{ }_{\mathcal{A}}$.

Restricting the dual map of $\tilde{\varphi}$ to $\mathcal{A}^{*}$ results in a completely positive map $\phi: \mathcal{A}^{*} \rightarrow \mathcal{B}(\mathcal{H})^{*}$ such that

$$
\omega(a)=\phi(\omega)(\iota(a)),
$$

where $\iota: \mathcal{A} \rightarrow \mathcal{B}(\mathcal{H})$ is the inclusion map. This implies that the composition of the dual map $\iota^{*}: \mathcal{B}(\mathcal{H})^{*} \mathcal{A}^{*}$ with $\phi$ is the identity map on $\mathcal{A}^{*}, \iota^{*} \circ \phi=\operatorname{id}_{\mathcal{A}^{*}}$. We note that the dual space of $\mathcal{B}(\mathcal{H})$ as well as $\mathcal{B}(\mathcal{H})$ itself admit nice finite-dimensional approximations, by restricting $\mathcal{H}$ to an increasing sequence of finite-dimensional subspaces. Hence it is desirable to understand the class of $\mathrm{C}^{*}$-algebras having the weak expectation property in more detail, in particular its functorial behavior with respect to quotients.

Quotient C*-algebras. We first recall the construction of quotient $\mathrm{C}^{*}$-algebras. If $\mathcal{A}$ denotes a $\mathrm{C}^{*}$-algebra, and $J \subset \mathcal{A}$ a (norm)-closed, two-sided ideal of $\mathcal{A}$, then $\mathcal{A} / J$ is itself a $\mathrm{C}^{*}$-algebra, see e.g. [83], I.8.1 and the canonical quotient map $q: \mathcal{A} \rightarrow \mathcal{A} / J$ is a *homomorphism. Unless otherwise noted, by an ideal in a $\mathrm{C}^{*}$-algebra we always mean a two-sided and norm closed ideal. We note that the dual space of $\mathcal{A} / J$ can be identified with the subspace of $\mathcal{A}^{*}$ which vanishes on the subalgebra $J$. This result can be strengthened in the case that $\mathcal{M}$ is a von Neumann algebra, and $J \subset \mathcal{M}$ a weak- ${ }^{*}$-closed two-sided ideal. Then there exists a central projection $e \in \mathcal{M} \cap \mathcal{M}^{\prime}$ such that $J \simeq(1-e) \mathcal{M}=(1-e) \mathcal{M}(1-e)$, see the first volume of the series by Takesaki, [83], Proposition II.3.12. This implies that the quotient $\mathcal{M} / J$ can be identified with $e \mathcal{M} e$, and the map $x \rightarrow$ exe is a normal conditional expectation $^{8}$, since the elements of $e \mathcal{M}_{*}$ vanish on $J$.

Like every $\mathrm{C}^{*}$-algebra is a subalgebra of $\mathcal{B}(\mathcal{H})$, there exists a corresponding universal object with respect to the quotient operation. We need again the free group $\mathbb{F}_{\infty}$ of countably many generators. The universal enveloping $\mathrm{C}^{*}$-algebra of $\mathbb{F}_{n}, C^{*}\left(\mathbb{F}_{n}\right)$, is the completion of the group ring of $\mathbb{F}_{n}$ with respect to $\|x\|=\sup _{\pi}\|x\|_{\mathcal{B}(\mathcal{H})}$, where the supremum is taken over all unitary representation of the free group into some $\mathcal{B}(\mathcal{H})$. It has the universal property that any *-homomorphism from the group ring into some $\mathcal{B}(\mathcal{H})$ extends to a *representation of $C^{*}\left(\mathbb{F}_{n}\right)$. Now let $\mathcal{A}$ be a separable $\mathrm{C}^{*}$-algebra, and let $\left(u_{i}\right)$ be a dense sequence of unitary elements. By the universal property of $C^{*}\left(\mathbb{F}_{\infty}\right)$, the map which associates the generator $g_{i}$ of $\mathbb{F}_{\infty}$ to $u_{i}$ extends to a $*$-representation $\pi: C^{*}\left(\mathbb{F}_{\infty}\right) \rightarrow \mathcal{A}$. Since $\pi$ is clearly onto it follows that $\mathcal{A} \simeq C^{*}\left(\mathbb{F}_{\infty}\right) / \operatorname{ker} \pi$. If $\mathcal{A}$ fails to be separable, then due to an analogous argument there is a nonetheless a free group $\mathbb{F}$ such that $\mathcal{A} \simeq C^{*}(\mathbb{F}) / J$, where $J \subset C^{*}(\mathbb{F})$ is an ideal.

The QWEP conjecture. If now $\mathcal{A} \simeq \mathcal{B} / J$, where $\mathcal{B}$ is a $\mathrm{C}^{*}$-algebra with the WEP, then we know from the preceding paragraph that there is a completely positive map from $\mathcal{B}^{*}$ into $\mathcal{B}(\mathcal{H})^{*}$. Furthermore, the corresponding dual map $E: \mathcal{B}(\mathcal{H})^{* *} \rightarrow \mathcal{B}^{* *}$ is a normal conditional expectation. Since $\mathcal{A} \simeq \mathcal{B} / J \subset \mathcal{B}^{* *} / J^{* *}$, we can find a central projection $e$ in the von Neumann algebra $\mathcal{B}^{* *}$ such that $\mathcal{A}^{* *} \simeq e \mathcal{B}^{* *}$ and $\mathcal{A}^{*} \simeq e \mathcal{B}^{*}$. It follows that we

\footnotetext{
${ }^{8} \mathrm{~A}$ map $E: \mathcal{N} \rightarrow \mathcal{M}$ between von Neumann algebras is called normal, if it is weak-*-continuous. It follows that the map admits a pre-dual, $E_{*}: \mathcal{M}_{*} \rightarrow \mathcal{N}_{*}$, and $\left(E_{*}\right)^{*}=E$.
} 
have a normal conditional expectation $E_{\mathcal{A}}: \mathcal{B}(\mathcal{H})^{* *} \rightarrow \mathcal{A}^{* *}$. The details of this reasoning can be found in [44]. This suggest that the class of $\mathrm{C}^{*}$-algebras having this property also admit certain finite-dimensional approximations. Following Kirchberg, who first studied this property, we say that a $\mathrm{C}^{*}$-algebra which is a quotient of a $\mathrm{C}^{*}$-algebra having the weak expectation property is QWEP. In his paper from 1996, where he introduced this class, Kirchberg also proved the following theorem, which asserts that being QWEP indeed corresponds to the existence of finite-dimensional approximations.

Theorem 2.2.7 (E.Kirchberg, [55]). Let $\mathcal{N}$ be a von Neumann algebra on a separable Hilbert space, which is a type $I I_{1}$ factor. Then $\mathcal{N}$ embeds into the ultrapower $(\mathcal{R})_{\mathcal{U}}$ of the hyperfinite type $I I_{1}$ factor if and only if it is $Q W E P$.

Because of this result, Kirchberg conjectured that all $\mathrm{C}^{*}$-algebras are QWEP. This is now called the $Q W E P$ conjecture. The equivalence to Connes' embedding problem follows from the permanence properties of the class of QWEP algebras, which we state for further reference. The proofs can be found in Kirchberg's paper, and in the excellent survey by Ozawa, [69].

Proposition 2.2.8. 1. Let $\mathcal{A}$ be a $C^{*}$-algebra. Then $\mathcal{A}$ is $Q W E P$ if and only if the double dual $\mathcal{A}^{* *}$ is $Q W E P$.

2. Let $\mathcal{I}$ be some index set and $\mathcal{A}_{i}$, for $i \in \mathcal{I}$ a collection of $C^{*}$-algebras such that for each $i \in \mathcal{I}, \mathcal{A}_{i}$ is $Q W E P$. Then the direct product $\prod_{i \in \mathcal{I}} \mathcal{A}_{i}$ is $Q W E P$.

3. If $\mathcal{A} \subset \mathcal{B}$, such that the $C^{*}$-algebra $\mathcal{B}$ is $Q W E P$, and there is a weak expectation from $\mathcal{B}$ to $\mathcal{A}$, then $\mathcal{A}$ is $Q W E P$.

4. A von Neumann algebra $\mathcal{N}$ is $Q W E P$ if and only if its commutant $\mathcal{N}^{\prime}$ is $Q W E P$.

5. A von Neumann algebra $\mathcal{N}$ is $Q W E P$ if and only if there is a separable Hilbert space $\mathcal{H}$ and a unital completely positive map from $\mathcal{B}(\mathcal{H})$ onto $\mathcal{N}$ which maps the closed unit ball of $\mathcal{B}(\mathcal{H})$ onto the closed unit ball of $\mathcal{N}$.

6. A von Neumann algebra $\mathcal{N}$ is $Q W E P$ if and only if there is a separable Hilbert space $\mathcal{H}$ such that $\mathcal{N}$ is isometrically isomorphic to a quotient Banach space of $\mathcal{B}(\mathcal{H})$.

Hence the class of QWEP $\mathrm{C}^{*}$-algebras is closed with respect to double duals, weak inductive limits and conditional expectations. From the theory of von Neumann algebras it then follows that if all type $I I_{1}$ factors are QWEP, then every $\mathrm{C}^{*}$-algebra is QWEP. Since every (separable) $\mathrm{C}^{*}$-algebra is a quotient of the universal $\mathrm{C}^{*}$-algebra of the free group, $C^{*}\left(\mathbb{F}_{\infty}\right)$, it is natural to ask whether $C^{*}\left(\mathbb{F}_{\infty}\right)$ has the weak expectation property.

Tensor product characterization. Since the weak expectation property can be characterized using tensor products, it is natural to ask for an equivalent reformulation of the QWEP conjecture in terms of tensor products. This is also due to Kirchberg, and follows from the following characterization of the WEP using the $\mathrm{C}^{*}$-algebra $C^{*}\left(\mathbb{F}_{\infty}\right)$.

Theorem 2.2.9 (E.Kirchberg, [55]). Let $\mathcal{A}$ be a $C^{*}$-algebra and let $C^{*}\left(\mathbb{F}_{\infty}\right)$ be the universal $C^{*}$-algebra of the free group of countably many generators. Then $\mathcal{A}$ has the weak expectation property if and only if

$$
\mathcal{A} \otimes_{\min } C^{*}\left(\mathbb{F}_{\infty}\right)=\mathcal{A} \otimes_{\max } C^{*}\left(\mathbb{F}_{\infty}\right) .
$$


Since $C^{*}\left(\mathbb{F}_{\infty}\right)$ can be approximated by its subalgebras $C^{*}\left(\mathbb{F}_{n}\right)$ for increasing $n$, and every such subalgebra admits a weak expectation, it is enough to consider the universal group $\mathrm{C}^{*}$ algebra for a finite number of generators. Hence Kirchberg's QWEP conjecture, equivalent to Connes' embedding conjecture, states now that there is also only one possible $\mathrm{C}^{*}$-norm on the tensor product of $C^{*}\left(\mathbb{F}_{n}\right)$ with itself, meaning that we have for all $n$

$$
C^{*}\left(\mathbb{F}_{n}\right) \otimes_{\min } C^{*}\left(\mathbb{F}_{n}\right)=C^{*}\left(\mathbb{F}_{n}\right) \otimes_{\max } C^{*}\left(\mathbb{F}_{n}\right) .
$$

Both Connes' and Kirchberg's conjecture are equivalent to a variety of different statements. We have only listed those which we think bear some connection to quantum information theory, or are at least understandable without relying on heavy machinery.

The aim of this work is to strengthen this connection between the existence of finitedimensional approximations and the aforementioned problem. However, we concentrate on more "physical" quantities than unitary operators and discuss physical properties of QWEP algebras. For example, we obtain similar conditions as in equation (2.8), but with the unitary elements replaced by operators characterizing measurement outcomes on arbitrary quantum systems.

For that it is necessary to consider not only $\mathrm{C}^{*}$-algebras, but subspaces of them, which contain the relevant physical operators. Such self-adjoint subspaces of a $\mathrm{C}^{*}$-algebra which contains the identity is called an operator system, and it is a special example of an archimedian $*$-ordered unit vector space. Both mathematical objects are introduced in the next chapter. 


\section{2 \\ Archimedian ordered vector spaces and Operator Systems}

This chapter is devoted to a short introduction into the theory of archimedian $*$-ordered vector spaces and operator systems. In short, the former are examples of ordered unit vector spaces, and the latter arise if we moreover consider tensor products with respect to matrix algebras. It is argued that these structures are mathematically intriguing in order to study physical theories. Most of the content of this chapter is taken from the excellent papers by Paulsen and coworkers [72, 71, 51, 52, 27], and the reader is referred to this literature for further details.

\subsection{Archimedian order unit spaces}

Ordered structures. The following exposition of archimedian $(*-)$ ordered vector spaces is mainly taken from the excellent paper by Tomforde and Paulsen, see [72]. Let $V$ be a complex vector space. A map $*: V \rightarrow V$ is called an involution, if $\left(v^{*}\right)^{*}=v$ and $(\lambda v+w)^{*}=\bar{\lambda} v^{*}+w^{*}$ holds for all $v, w \in V, \lambda \in \mathbb{C}$, where $\bar{\lambda}$ is the complex conjugate of $V$. The real vector space $\left\{v \in V: v^{*}=v\right\}$ is called the set of hermitian elements $V_{h}$ in $V$. Every element $v \in V$ can be uniquely decomposed as $v=x+i y$ with $x, y \in V_{h}$. Following [72], we call $x$ the real and $y$ the imaginary part of $v$. Note also that this implies the isomorphic identification

$$
V \cong V_{h} \oplus i V_{h} .
$$

Definition 3.1.1. A full cone $V_{+}$in $V$ is a convex subset of $V_{h}$ such that $V_{+} \cap-V_{+}=\{0\}$ and every element $v$ of $V_{h}$ can be written as $v=a-b$, for some elements $a, b \in V_{+}$. The cone $V_{+}$introduces a partial order on $V$ be the formula $v \geq w \leftrightarrow v-w \in V_{+}$. $V_{+}$is also called the set of positive elements, since any element $v \in V_{+}$fulfills $v \geq 0$. An order unit is an element $e \in V_{+}$such that for every element $v \in V_{h}$ there exists a positive number $r$ such that $r e-v \geq 0$. The triple $\left(V, V_{+}, e\right)$ is called a $*$-ordered vector space. The order unit is called archimedian, if $r e+v \geq 0, \forall r>0$ implies $v \in V_{+}$. An archimedian $*$-ordered 
vector space is a triple $\left(V, V_{+}, e\right)$ such that $V_{+}$is a full cone in the complex vector space $V$ and $e$ is an archimedian order unit for $V_{+}$. We abbreviate such a triple by the expression *-AOU space.

The set of hermitian elements $V_{h}$ is a real vector space, and can be employed with a semi-norm induced by the order. More formally, we set for an element $v \in V_{h}$

$$
\|v\|=\inf \{r>0:-r e \leq v \leq r e\} .
$$

This seminorm is called the order seminorm, and it is a norm if $V_{h}$ and hence $V$ is an archimedian ordered vector space $[72,1]$. In that case, we call the order seminorm the order unit norm. A norm on an archimedian $*$-ordered vector space $V$ is called order norm, if it coincides with the order unit norm on the set of hermitian elements. It follows from the work of Paulsen and Tomforde that all order norms on a *-AOU space are equivalent, see Proposition 4.9 in [72].

Given a $*$-ordered vector space $\left(V, V_{+}, e\right)$, a linear complex valued functional $f: V \rightarrow \mathbb{C}$ is called positive if $f\left(V_{+}\right) \subset \mathbb{R}_{+}$, the set of positive real numbers. We denote the set of positive functionals on $V$ by $V_{+}^{*}$. Positive functionals $f$ with the additional property that $f(e)=1$ are called states, and we denote the set of states by $S(V)$. If $e$ is archimedian, then the set of states characterize positivity in the following sense.

Proposition 3.1.1 (Paulsen and Tomforde, [72]). Let $\left(V, V_{+}, e\right)$ be a $*$-AOU space, and $v \in V$. Then $v$ is positive if and only if $\omega(v) \geq 0$ for all states $\omega \in S(V)$. Moreover, if $\omega(v)=0$ for all $\omega \in S(V)$, it follows that $v=0$.

The order seminorm can be expressed using the set of states, namely we have for $v \in V_{h}$

$$
\|v\|=\sup \{|\omega(v)|: \omega \in S(V)\} .
$$

The extension of this norm to all of $V$ is called the minimal order (semi)-norm, see [72] for details. If $\left(V, V_{+}, e\right)$ is an archimedian $*$-ordered vector space equipped with an order norm $\|$.$\| , then we denote by V^{*}$ the set of linear, complex valued functional on $V$ which are continuous with respect to the norm topology. For $f \in V^{*}$ we set $\|f\|=\sup \{|f(x)|$ : $a \in V,\|a\|=1\}$. We have the following relationship between positivity conditions and norm bounds for $f$.

Lemma 3.1.2 ([72], Proposition 2.28, Lemma 4.16). Let $\left(V, V_{+}, e\right)$ be an archimedian *ordered vector space equipped with an order norm $\|$.$\| and let f: V \rightarrow \mathbb{C}$ be a complex valued functional on $V$. Then $f$ is positive if and only if it is continuous with respect to the norm topology and we have that $\|f\|=f(e)$.

The dual $V^{*}$ of a real archimedian ordered vector space $V$ is known to be a base-norm space. That is, there exist a compact convex subset $K \subset V^{*}$ such that $C=\cup_{\lambda \geq 0} \lambda K$ is a full cone for $V^{*}, V^{*}$ is complete in the norm topology induced by the Minkowski functional

$$
\|f\|=\inf \{\lambda \geq 0: x \in \lambda \operatorname{conv}(K \cup-K)\}
$$

and the unit ball is equal to $\operatorname{conv}(K \cup-K)$. Moreover, every element $f \in V^{*}$ admits a decomposition $f=a-b$, where $a, b$ are positive functionals and $\|f\|=\|a\|+\|b\|$. For more informations and proofs of these statements see the book by Alfsen, [1], chapter II.1. Since the hermitian subspace $V_{h}$ of a $*$-AOU space is a real archimedian ordered vector space, these results can be used for real valued functionals on $V_{h}$. 
Definition 3.1.2. Let $\left(V, V_{+}, e_{V}\right)$ and $\left(S, S_{+}, e_{S}\right)$ be two $*$-AOU spaces. A linear map $T: V \rightarrow S$ is called hermitian if it maps hermitian elements to hermitian elements. Correspondingly, it is called positive if $T\left(V_{+}\right) \subset S_{+}$. It is called unital, if $T\left(e_{V}\right)=e_{S}$. If a unital linear map $T$ is invertible, and its inverse is again positive, then we call $T$ an order isomorphism. Two $*$-AOU spaces $S$ and $V$ are called isomorphic, if there exist an order isomorphism between $S$ and $V$. We note that a unital linear and invertible map $T: V \rightarrow S$ is an order isomorphism when $v \in V_{+}$is equivalent to $T(v) \in S_{+}$. If $T$ is injective, but not surjective, and its inverse defined on the range of $T$ is positive, then we call $T$ an order isomorphism onto its range.

Note that if $T$ is an order isomorphism onto its range, then it has to be also unital, due to the fact that the order units are archimedian. In addition, by equal reasoning it has to be continuous with respect to any order norm ${ }^{1}$. In the following, we assume that all our *-AOU spaces can be equipped with a norm which is compatible to the respective unit, and all maps, if not noted otherwise, are taken to be continuous with respect to that norm topology.

Example 3.1.1. Let $K$ be some compact subset of some topological space, and consider the space $C(K)$ of complex-valued continuous functions $f: K \rightarrow \mathbb{C}$. The involution is defined canonically as $f^{*}(x)=f(x)^{*}$, for $x \in K$. A function is called positive, $f \in C(K)_{+}$ if $f(x) \geq 0$ for all $x \in K$. Since $K$ is compact, the function $1_{K}$ which takes the value one on all of $K$ is continuous, and it is easily checked that it is also an archimedian order unit for the $*$-ordered vector space $\left(C(K), C(K)_{+}\right)$. Now consider a self-adjoint subspace $V$ of $C(K)$, i.e. a subspace such that if $f \in V$ then also $f^{*} \in V$. Furthermore, assume that the identity function $1_{K}$ is also an element of $V$. Then $V_{+} \equiv V \cap C(V)_{+}$is a full cone for the vector space $V$, and $1_{K}$ is again an archimedian order unit. The following theorem asserts that every $*$-AOU space arises in such a fashion.

The following theorem is the generalization of Kadison's characterization of subspaces containing the identity function of real-valued continuous functions. In its original form, *-AOU spaces are replaced by real archimedian ordered unit vector spaces.

Theorem 3.1.3 (Paulsen and Tomforde, [72]). Let $\left(V, V_{+}, e\right)$ be an $*$-AOU space. Then there exist a compact subset $K$ of some Hausdorff space, and a positive and unital map $T: V \rightarrow C(K)$ which is an order isomorphism onto its range. In particular, $K$ can be taken to be equal to the state space of $V$ with the weak-*-topology, and the map $T$ is defined as

$$
T(v)(\omega) \equiv \omega(v)
$$

for $\omega \in S(V) \simeq K$.

The proof idea is as follows. First, general considerations imply that the state space of $V$ is compact in the weak- ${ }^{*}$-topology ${ }^{2}$. This can be seen from the fact that any net of positive functional converges to a positive functional. Defining the map $T$ as in equation (3.2), it is obvious that $T$ is positive. Moreover, if $T(v)=0$, then $\omega(v)=0$ for all states $\omega$, and hence $T$ is injective. This fact as well as the positivity of the inverse of $T$ (defined

\footnotetext{
${ }^{1}$ Note that all order norms are equivalent, as emphasized earlier.

${ }^{2}$ The topology defined by the seminorms $\omega \mapsto \omega(v)$ for $v \in V$. Clearly, we can restrict to the hermitian elements.
} 
on its range) follow both from Proposition 3.1.1. We note that this is not longer true if $e$ fails to be an archimedian order unit.

We note that though any *-AOU space can be realized as a subspace of $C(S(V))$, it is most often not the case that $V \simeq C(K)$, for some compact set $K$. For real archimedian ordered vector spaces, this requirement forces $V$ to be a lattice ${ }^{3}$, and $S(V)$ to be a simplex. Indeed, if we assume for the moment that $V$ is finite-dimensional, then it is easy to see that it in the definition of the map $T$ in equation (3.2), it is enough to consider only the extreme points $\partial S(V)$ in $S(V)$. Note that in the finite-dimensional case, the fact that the state space is a simplex implies that there are only finitely many extreme points in $S(V)$. This follows from Caratheodory's theorem. Since any state is a convex combination of extreme points of $S(V)$, which are also called pure states, an element $v \in V$ is positive if and only if $\omega(v) \geq 0$ for all $\omega \in \partial S(V)$. By the same fact, it follows that a function which is continuous on the extremal states ${ }^{4}$ can be extended to a linear map on all of $S(V)$. In the infinite-dimensional case, when $V$ is a lattice, or equivalently, $S(V)$ is a simplex, then it can be shown that the set of extreme points of $S(V)$ is itself weak-*-closed, and moreover the finite-dimensional reasoning carries over, that is we have $V \simeq C(\partial S(V))$. See the book of Alfsen [1] for details on this.

The following example is familiar to all physicists.

Example 3.1.2. Consider the vector space $M_{n}$ of $n \times n$ complex valued matrices, and take $\mathbb{I}_{n}$ to be the identity matrix on the complex vector space $\mathbb{C}^{n}$. The involution on $M_{n}$ is taken to be the complex conjugation of matrices, i.e. $\left(m_{i j}\right)^{*}=\left(m_{j i}^{*}\right)$. As usual we call an element $M \in M_{n}$ positive if $\langle\psi \mid M \psi\rangle \geq 0$, for every element $\psi \in \mathbb{C}^{n}$ (note that it is enough to consider only vectors of norm equal to one), and $\langle. \mid$.$\rangle denotes the usual scalar$ product of $\mathbb{C}^{n}$. The set $M_{n,+}$ of positive matrices $M \geq 0$ form a cone in $M_{n}$ and we know that a hermitian matrix $M$ is positive if and only if all its eigenvalues are positive. We obviously have that by adding a suitable scaled multiple of the identity, every hermitian element of $M_{n}$ becomes positive. Moreover, since $M_{n}$ is a finite-dimensional vector space, the condition

$$
\varepsilon\left\langle\psi \mid \mathbb{I}_{n} \psi\right\rangle-\langle\psi \mid M \psi\rangle=\varepsilon-\langle\psi \mid M \psi\rangle \geq 0 \quad \forall \varepsilon>0, \forall \psi \in \mathbb{C}^{n},\langle\psi \mid \psi\rangle=1
$$

implies that $\langle\psi \mid M \psi\rangle \geq 0$. It follows that the identity matrix $\mathbb{I}_{n}$ on $\mathbb{C}^{n}$ is an archimedian order unit for the $*$-ordered vector space $\left(M_{n}, M_{n,+}\right)$. The set of positive functionals on $M_{n}$ can again be identified with the set of positive matrices, under the duality

$$
\omega_{\rho}(M)=\operatorname{Tr} \rho M
$$

for $\rho, M$ in $M_{n,+}$. It follows that $\rho$ belongs to a state if $\operatorname{Tr} \rho=1$. Such an element is also called a density operator. Consider now the space $C(\rho)$ of continuous complex valued functions on the set of density operators. Clearly, the space $M_{n}$ embeds into $C(\rho)$, but it is only a subspace of it, as the range of the embedding consists of only linear functions. This example basically characterizes the theory of quantum mechanical systems with finitedimensional state space.

\footnotetext{
${ }^{3}$ A lattice is a partially ordered set such that for any two elements, there exist a least upper bound, as well as a greatest lower bound. For more information on ordered vector spaces which are also lattices, see [80].

${ }^{4}$ As there are only finitely many extreme points, the topology used here is the trivial point-topology.
} 
Physical theories. It follows from these examples that the structure of $*$-AOU spaces is often not sufficient in order to differentiate physical theories. That is, on the ground of its structure as an $*$-AOU space, quantum mechanics looks as it would be a subspace of the space of complex valued functions, defined on its state space. The connection between *-AOU spaces and physical theories is illustrated by the next example. But first, it helps to recall another results of Paulsen and Tomforde.

For that, note that a $*$-ordered vector space can fail to be archimedian due to basically two facts. First, the cone can be open in the order topology, which contradicts the fact that

$$
r e+v \geq 0, \forall r>0 \Rightarrow v \in V_{+}
$$

or the state space is "too small", meaning that the fact that $\omega(v)=0$ for all $\omega \in S(V)$ does not imply $v=0$. Both circumstances can occur, but can be overcome by a technique called the Archimedization of a *-ordered vector space. This process basically addresses both issues separately, by first closing the cone and then taking the quotient of $V, V / N$ with respect to the subspace $N$ of all elements which cannot be differentiated by the set of states. The result is an $*$-AOU space which is uniquely defined by its originating space. For details, see [72].

We now sketch the usefulness of the formalism of ordered vector spaces in the search for an axiomatic derivation of quantum mechanics first started by Ludwig, Mittelstedt and others $[59,60,63]$. They were among the first to realize that reasonable physical theories should allow for the convex combination of different possible measurements or states, and hence the underlying sets of both states and measurements should be convex bodies which are in duality. However, these attempts concentrated on the single state space, and mostly did not study joint systems. This changed in the last decade, where several authors put forward an information-theoretic axiomatic characterization of the laws of nature: beginning with Hardy's influential paper [41] there has been a sequence of developments leading to the identification of general probabilistic theories (GPT) as a general framework to study theories of physics going beyond quantum mechanics $[5,6,7,61]$.

A physical state assigns a probability for every measurement outcome, i.e., a state $s$ is a map from the set of effects to $[0,1]$. We denote the set of effects by $A_{+1}$, and the set of states by $S(A)$. This map should respect probabilistic mixtures of states, meaning that if $s=p s_{1}+(1-p) s_{2}, p \in[0,1]$, and $a$ is an effect then $\left(p s_{1}+(1-p) s_{2}\right)(a)=$ $p s_{1}(a)+(1-p) s_{2}(a)$, i.e., the map is affine. Similarly, if $a$ and $b$ are effects, then we require that $s(p a+(1-p) b)=p s(a)+(1-p) s(b)$. This is exactly the setting of two convex sets in duality. The space of all unnormalised effects may be given the structure of a convex cone $A_{+}$by formally defining all positive linear combinations $\sum_{j=1}^{m} \lambda_{j} a_{j}$, where $\lambda_{j} \in \mathbb{R}_{+}$and $a_{j} \in A_{+}$, to be themselves possible unnormalised effects. A special effect is the unit effect $e$, which is uniquely defined such that $s(e)=1$ for all states $s$. The unit effect represents the "do nothing" measurement with a single outcome that is certain to occur regardless of the state.

An observable $O$ is then given as a collection of effects, $a_{O, i} \in A_{+1}$, where $a_{O, i}$ represents the effect corresponding to outcome $i$ of the observable $O$. Since we do not want to discard any outcome, the sum of the $a_{O, i}$ should be the "do nothing" measurement, $\sum_{\alpha} a_{O, i}=$ $e$, also providing the normalization condition for the outcome probabilities to hold. As indicated, the "do nothing" measurement is unique, and hence the sum is equal to the unit effect for all observables. 
Because of this linear nature, it is mathematically intriguing to also consider the algebraic span over the complex numbers of effects. We denote the resulting complex vector spaces by $A$. It follows that $A_{+} \equiv\left\{\lambda \cdot A_{+1} \mid \lambda \in \mathbb{R}_{+}\right\}$is a full cone in $A$, which introduces a partial order by $x \leq y$ if and only if $x-y \in A_{+}$. The involution is then defined canonically as

$$
\left(\sum_{i} c_{i} a_{i}\right)^{*}=\sum_{i} c_{i}^{*} a_{i}
$$

for effects $a_{i} \in A_{+}$and complex numbers $c_{i} \in \mathbb{C}$. This turns the vector space $V$ together with its cone into a $*$-ordered vector space. Since the unit effect $e$ is necessarily an interior element of $A_{+}$, there exist for every element $v$ in $V$ a real number $r>0$ such that $r e \geq v$. Hence $e$ is an order unit for the $*$-ordered vector space $\left(A, A_{+}\right)$. Its archimedization is nothing else but taking the quotient with respect to the equivalence relation inferred from the set of states

$$
a \sim b \Leftrightarrow \omega(a-b)=0 \forall \omega \in S(A) .
$$

The closure of the cone can be understood as the enlargement of the cone of effects with all "limit" observables. We arrive at the $*$-AOU space $\left(A, A_{+}, e\right)$.

If now Alice and Bob are two spacelike separated observers, we denote by $A_{+1}\left(B_{+1}\right)$ the set of measurement effects on Alice' (resp. Bob) side. A state on the joint system is then first of all nothing else but a map

$$
\omega: A_{+1} \times B_{+1} \rightarrow[0,1]
$$

associating to each combination of Alice' and Bob choice of effects a probability. The "do nothing" measurement on the joint system should naturally correspond to "doing nothing" on both individual systems, so if $e_{A}$ and $e_{B}$ denote Alice' and Bob's unit effects, then we require $\omega\left(e_{A}, e_{B}\right)=1$. Now, if Alice and Bob each choose a finite set (say $n$ ) of effects, $a_{i} \in A_{+}, b_{j} \in A_{+}, i, j \in\{1, \ldots, n\}$, then $\omega\left(a_{i}, b_{j}\right)$ is an $n \times n$ matrix with positive entries, which we call correlation matrix $C_{\omega}$. The "quality" of correlation between two spacelike separated observers, i.e. whether they obey classical/quantum statistical behavior, or even exceed the possible set of correlations of these two theories, is measured by Bell like tests, see for example $[8,9,16,90,28]$. Such a correlation test $T$ is given by a linear functional on the set of correlation matrices, hence itself is determined by a real valued matrix,

$$
T\left(C_{\omega}\right)=\sum_{i j} T_{i j} \omega\left(a_{i}, b_{j}\right) .
$$

That linear correlation tests are indeed sufficient in order to characterize physical theories can be seen from the fact the the set of correlation matrices (for a given theory) is clearly convex. By the Hahn-Banach separation theorem, there exists a linear functional separating this set from its outset.

The first kind of this test was proposed 1964 by John Bell, in order to experimentally verify that quantum mechanics indeed correctly describes the physics of microscopic particles. The term Bell inequality refers to the fact that Bell and later Clauser, Horne, Shimony and Holt constructed a $T$ such that the value of $T\left(C_{\omega}\right)$ was bounded (say by 1 ) for all classical correlation matrices and thus any violation of this bound proves that classical theory is not enough to explain certain correlations. 
Every $T$ is a bilinear map on the cartesian product $A \times B$ of two $*$-AOU spaces. Furthermore, from physical considerations we have to require the so-called no-signaling condition, that is, the choice of Alice' observable should not influence the choice on Bob's side. Since an observable is given as a collection of elements $a_{O, i} \in A_{+}$, representing the outcome $i$ of the observable $O$, this physical condition translates into the requirement that if we sum up the outcomes on Alice side, then the corresponding joint effect must correspond to the case that Alice "does nothing", i.e. measures the unit effect, and Bob's effect is untouched. Mathematically, we want to linearize the cartesian product $A_{+} \times B_{+}$. The tensor product of the vector spaces does exactly that, since the linear normalization relation survives in the tensor product as

$$
\sum_{i} a_{O, i} \otimes b_{j}=e \otimes b_{j}
$$

Hence the non-signaling condition translates to the requirement that $\omega$ extends to a positive $\left(\omega\left(A_{+}, B_{+}\right) \subset \mathbb{R}_{+}\right)$, bilinear map. This extension is defined because we assume that there is a linear rule to combine two physical systems, or tensor two *-AOU spaces together, which is apart from the convexity of the set of effects the second assumption for GPT's. The properties of $\omega$ imply that every effect $a_{i}$ on Alice' side can be used to define a map $f_{\omega, a_{i}}(c)=\omega\left(a_{i}, c\right)$ for $c \in V_{B}$ which is itself positive $\left(f_{\omega, a_{i}}\left(A_{+}\right) \subset \mathbb{R}_{+}\right)$and linear, i.e. it is a positive linear functional. This example motivates the study of tensor products between *-AOU spaces.

Tensor products and joint systems. We are given two $*$-AOU spaces $\left(A, A_{+}, e_{A}\right)$ and $\left(B, B_{+}, e_{B}\right)$. We let $A \otimes B$ be its algebraic tensor product, i.e. the linear span of all elementary tensors $a \otimes b$ for $a \in A$ and $b \in B$. For illustration, we might identify them with two physical systems, called Alice and Bob, each characterized as in the preceding discussion. From these considerations, their tensor product should be identified with the joint system. In order to characterize the associated $*$-AOU space, we have to specify a positive cone $C_{A \otimes B}$ in $A \otimes B$, together with an archimedian order unit. In doing so, several requirements should be met.

(i) If Alice and Bob both perform a measurement, i.e. measure the effect $a \in A_{+}$and $b \in B_{+}$, then the joint measurement $a \otimes b$ should also represent an effect. Hence we require

$$
\forall(a, b) \in A_{+} \times B_{+} a \otimes b \in C_{A \otimes B} .
$$

(ii) If both parties "do nothing", then this should be the "do nothing" measurement on the joint system. Hence $e_{A} \otimes e_{B}$ should be the archimedian order unit for $C_{A \otimes B}$.

These requirements derived from physical reasoning motivate the following definition.

Definition 3.1.3. Let $\left(A, A_{+}, e_{A}\right)$ and $\left(B, B_{+}, e_{B}\right)$ be two $*$-AOU spaces, and let $A \otimes B$ be their algebraic tensor product. A cone $C_{A \otimes B}$ in $A \otimes B$ is called a tensor product cone, if it satisfies conditions (i) to (ii) in the above listing. The $*$-AOU space $\left(A \otimes B, C_{A \otimes B}, e_{A} \otimes e_{B}\right)$ is called a tensor product of $*$-AOU spaces.

From the requirements above, two natural choices of tensor product cones are immediate. The first is, loosely speaking, the "biggest" possible cone, whereas the second is the "smallest possible" cone. Note that different to these statements, the first is called the "minimal" 
tensor product, whereas the second is called the "maximal" tensor product, related to the fact that for the associated state spaces, the inverse relation is true.

Definition 3.1.4. Let $\left(A, A_{+}, e_{A}\right)$ and $\left(B, B_{+}, e_{B}\right)$ be two $*$-AOU spaces, let $S(A)$ respectively $S(B)$ their associated state spaces and let $A \otimes B$ be their algebraic tensor product. The minimal tensor product cone $C_{\text {min }}$ is defined as

$$
C_{\text {min }} \equiv\{z \in A \otimes B \mid \omega \otimes \sigma(z) \geq 0 \forall(\omega, \sigma) \in S(A) \times S(B)\} .
$$

If $\left(V, V_{+}, e_{V}\right)$ is another $*$-AOU space, and $\Omega: A \times B \rightarrow V$ is a bilinear map, then its linear extension to all of $A \otimes B$ called jointly positive, if we have that $\Omega(a, b) \in V_{+}$for all $a \in A_{+}$, $b \in B_{+}$. The maximal tensor product cone $C_{\max }$ is defined as

$$
C_{\max } \equiv\{z \in A \otimes B \mid \Omega(z) \geq 0 \text { for all jointly positive maps } \Omega: A \otimes B \rightarrow \mathbb{C}\} .
$$

A proof that these are indeed tensor product cones can be found in [38]. We see that the state space of the maximal tensor product cone is the largest possible, as any state on the joint system should clearly be a jointly positive map. Correspondingly, the minimal tensor product cone corresponds to the smallest state space, that is a joint action $z \in A \otimes B$ is positive, if its evaluation on all convex combinations of product states gives rise to a positive value. Conversely, the maximal tensor product cone can also be described directly in terms of the cones $A_{+}$and $B_{+}$, namely we have, see [38],

$$
C_{\max }=\left\{z \in A \otimes B \mid \forall \varepsilon>0: \varepsilon e_{A} \otimes e_{B}+z \in \operatorname{conv}\left(A_{+} \otimes B_{+}\right)\right\} .
$$

Here, conv denotes the convex hull, and $A_{+} \otimes B_{+}$is the set of all tensors $a \otimes b, a \in A_{+}$, $b \in B_{+}$. From these characterizations, it is then obvious that the minimal and the maximal tensor product cones are the biggest resp. the smallest possible tensor product cone, that is if $C_{A \otimes B}$ is any tensor product cone than we have

$$
C_{\max } \subset C_{A \otimes B} \subset C_{\min } .
$$

The case that there is only one possible tensor cone on the algebraic tensors product $A \otimes B$, i.e. $C_{\min }=C_{\max }$ was first considered, up to our knowledge, in the case of real ordered vector spaces by Namioka and Phelps, [65]. The definitions of the tensor cones is analogous in this case. Their theorem 1.4 states that $\left(A, A_{+}, e_{A}\right)$ is an order unit vector space such that the minimal and the maximal tensor product cone coincide for all order unit spaces if and only if the state space $S(A)$ is a simplex. As discussed earlier, this means that $A \simeq C(\partial S(A))$. By Uryson's Lemma, each element of $A$ can then be approximated by a linear combination of continuous functions which are almost constant. After some work, it is seen that we can take these approximations as the restriction of elements of $A$ onto a finite set of (extremal) states on $A$. This results in the $*$-AOU space $\ell_{n}^{\infty}$, i.e. the vector space of all complex valued $n$-tuples where positivity is defined entry-wise and the identity element has all entries equal to one.

This reasoning was generalized to complex case by Han [38]. He found that for a $*$-AOU space $\left(A, A_{+}, e_{A}\right)$, the minimal and the maximal tensor product cone coincide on the joint system $A \otimes B$ for any other $*$-AOU space $\left(B, B_{+}, e_{B}\right)$ if and only if there exists a net of pairs of positive maps

$$
u_{\varepsilon}: V \rightarrow \ell_{n(\varepsilon)}^{\infty} \quad v_{\varepsilon}: \ell_{n(\varepsilon)}^{\infty} \rightarrow V
$$


such that the following diagram approximately commutes in the point-norm topology with respect to any (and hence all) order norms.

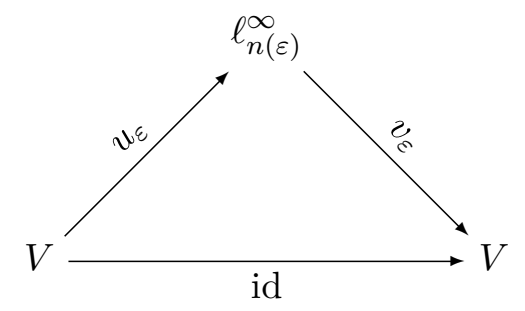

By approximately commuting, we mean that $\left\|v_{\varepsilon} \circ u_{\varepsilon}(v)-v\right\| \leq \varepsilon\|v\|$, for all $\varepsilon>0$ and $v \in V$. We call such a $*$-AOU space nuclear in the category of $*$-AOU spaces $^{5}$. Although it was proven in this form just lately, the reader should take it as the first indication that tensor products and approximations by prime examples such as the $*$-AOU space $\ell_{n}^{\infty}$ are intimately related.

It follows from the above results, but also from direct considerations, that the $*$-AOU space $\ell_{n}^{\infty}$ is nuclear. Another prime example from physics is the $*$-AOU space a complex valued matrices, as explained above. If we take now two copies of it, and consider their minimal and maximal tensor product cone, then it is easily seen that they are disjoint. Indeed, the minimal tensor product cone consists of all elements of $M_{n} \otimes M_{n} \simeq M_{n^{2}}$ which are positive on product states. These elements are also called entanglement witnesses. On the other hand, there exist complex valued jointly positive maps on $M_{n} \otimes M_{n}$ which cannot be written as a convex combinations of product states. These are called entangled states, [89]. Hence, the space describing finite-dimensional quantum systems is not nuclear in the category of $*$-AOU spaces. However, we would certainly want that we can define the notion of joint systems if one of the physical subsystems is described by quantum mechanics. If we furthermore require that this notion is unique for some $*$-AOU space $\left(V, V_{+}, e\right)$, then we arrive at the setting of operator systems. This is the topic of the next section.

\subsection{Operator systems}

Definitions. Operator systems can be seen as $*$-AOU spaces equipped with a notion of joint systems, where the second system is a finite-dimensional quantum system. As described in the last section, a finite-dimensional quantum system is described by the complex vector space $\mathbb{C}^{n}$, where $n \in \mathbb{N}$ is the number of degrees of freedom.

In the following we denote by $\left\{\left|\xi_{i}\right\rangle\right\}_{i \in\{1, \ldots, n\}}$ an orthonormal bases of the vector space $\mathbb{C}^{n}$. The rank one matrices $\left|\xi_{i}\right\rangle\left\langle\xi_{j}\right|$ form a basis of the $\mathrm{C}^{*}$-algebra of $n \times n$ complex matrices $M_{n}$. Hence any element $v$ of the algebraic tensor product $M_{n} \otimes V$ between a $*$-AOU space $V$ and $M_{n}$ can be represented as $v=\sum_{i j} v_{i j} \otimes\left|\xi_{i}\right\rangle\left\langle\xi_{j}\right|$. In the case of $V \cong \mathbb{C}$ we will omit the tensor product sign. Naturally, the vector space $M_{n} \otimes V$ can be identified with the set of all $n \times n$ matrices with entries in $V$. It can also be employed with an involution, setting $\left(\sum_{i j} v_{i j} \otimes\left|\xi_{i}\right\rangle\left\langle\xi_{j}\right|\right)^{*}=\sum_{i} v_{i j}^{*} \otimes\left|\xi_{j}\right\rangle\left\langle\xi_{i}\right|$. If $K \in M_{n}, v \in M_{n} \otimes V$ and $\mathbb{I}_{V}$ denotes the identity map on $V$, then we set

$$
\left(K \otimes \mathbb{I}_{V}\right) v=\sum_{i j} v_{i j} \otimes\left(K \cdot\left|\xi_{i}\right\rangle\left\langle\xi_{j}\right|\right),
$$

\footnotetext{
${ }^{5}$ Note the similarity to nuclear $\mathrm{C}^{*}$-algebras. In a broad sense, an element of a category is called nuclear, if there is only one tensor product possible which preserves the features of the category.
} 
where is the usual matrix product on $M_{n}$. As we know from quantum mechanics, a physical possible operation on a finite-dimensional quantum system is a map $T: M_{n} \rightarrow M_{m}$ which can be written as

$$
T(x)=\sum_{i} K_{i}^{*} x K_{i}
$$

where $K$ maps the Hilbert space $\mathbb{C}^{m}$ to $\mathbb{C}^{n}$, i.e. it is a rectangular complex-valued matrix. We usually also require that $T$ is unital, i.e. $\sum_{i} K_{i}^{*} K_{i}=\mathbb{I}_{m}$. If this is the case, then $T$ can be alternatively written as

$$
T(x)=u^{*} x \otimes \mathbb{1}_{p} u,
$$

where $p \leq n^{2}$ and $u: \mathbb{C}^{m} \rightarrow \mathbb{C}^{m} \otimes \mathbb{C}^{p}$ is an isometry. Here, $\mathbb{I}_{p}$ denotes the identity matrix on the complex vector space $\mathbb{C}^{p}$. Obviously, this is just Stinespring's dilation theorem applied to finite-dimensional matrix algebras. If we now consider the joint system of some *-AOU space together with some finite-dimensional quantum system, then it is reasonable to require that physical possible operations on the quantum system (while doing nothing on the second system) extend to physical possible operations on the joint system. In particular, this should be the case if degrees of freedoms are added or reduced. In view of the study of tensor products of $*$-AOU spaces, this motivates the following definitions.

Definition 3.2.1. Let $C_{n}$ be a family of full cones in the complex vector spaces $M_{n} \otimes V$, $C_{n} \subset M_{n} \otimes V$. We call the family $C_{n}$ compatible, if $C_{1}=V_{+}$and for every complex valued $n \times m$ matrix $K$ it holds that

$$
\left(K^{*} \otimes \mathbb{I}_{V}\right) C_{n}\left(K \otimes \mathbb{I}_{V}\right) \subset C_{m} .
$$

A *-ordered vector space $\left(V, V_{+}\right)$equipped with a compatible family of cones $C_{n} \subset M_{n} \otimes V$ is called a $*$-matrix ordered space ${ }^{6}$. Correspondingly, a compatible family of cones $C_{n}$ is called archimedian, if $e_{n}=\mathbb{I}_{n} \otimes e$ is an archimedian order unit for all cones $C_{n}, n \in \mathbb{N}$. Let now $\left(V, V_{+}, e\right)$ be an archimedian *-ordered vector space and $C_{n} \subset M_{n} \otimes V$ be an archimedian compatible family of cones. Then we call the triple $\left(V,\left\{C_{n}\right\}, e\right)$ an operator system.

If the family of cones is clear from the context, we also often write $\left(M_{n} \otimes V\right)_{+}$for the family of matrix cones of $V$. Instead of writing $M_{n} \otimes V$ we also use the shorthand notation $M_{n}(V)$, and $M_{n}(V)_{+}$instead of $\left(M_{n} \otimes V\right)_{+}$. Like in the case of $*$-AOU spaces, we next consider the morphisms in the category of operator systems. As the defining property are the matricial cones over $V$, the following definition is obvious.

Definition 3.2.2. A linear map $T: V \rightarrow W$ between to $*$-matrix ordered systems is called completely positive, if the map

$$
\mathbb{I}_{n} \otimes T: M_{n} \otimes V \rightarrow M_{n} \otimes W, \quad \mathbb{I}_{n} \otimes T\left(\sum_{i j} v_{i j} \otimes\left|\xi_{i}\right\rangle\left\langle\xi_{j}\right|\right)=\sum_{i j} T\left(v_{i j}\right) \otimes\left|\xi_{i}\right\rangle\left\langle\xi_{j}\right|
$$

is positive on all matrix levels $n \in \mathbb{N}$. We denote the set of completely positive maps between operator systems $V$ and $W$ by $C P(V, W)$. A bijective unital and completely positive

\footnotetext{
${ }^{6}$ This especially implies that the notion of positivity is independent of the basis choice $\left\{\left|\xi_{i}\right\rangle\right\}_{i \in\{1, \ldots, n\}}$. Moreover, it follows from the study of tensor products of $*$-AOU spaces that if $e \in V$ is an order unit, then the element $\mathbb{I}_{n} \otimes e$ is an order unit for $M_{n} \otimes V$.
} 
map $T \in C P(V, W)$ such that its inverse $T^{-1}$ is also positive is called a complete order isomorphism. Correspondingly, two operator systems are called completely isomorphic if there exists a complete order isomorphism between them. If $T: V \rightarrow W$ is an injective completely positive unital map such that the inverse defined on its range is completely positive, then $T$ is called a complete order isomorphism onto its range. Alternatively, we also call $T$ a complete order embedding. If $V$ and $W$ is a pair of operator systems such that there exits a complete order embedding from $V$ to $W$, then we write $V \subset W$.

Dual space. The matricial dual space $M_{n} \otimes V^{*}$ can also be equipped with a family of cones by defining an element $\sum_{i j} \omega_{i j} \otimes\left|\xi_{i}\right\rangle\left\langle\xi_{j}\right| \in M_{n} \otimes V^{*}$ to be positive if the map

$$
\hat{\omega}: V \rightarrow M_{n}, \quad \hat{\omega}(v)=\sum_{i j} \omega_{i j}(v)\left|\xi_{i}\right\rangle\left\langle\xi_{j}\right|
$$

is completely positive. We denote this set of positive cones in $M_{n} \otimes V^{*}$ by $C_{n}^{*}$. It was shown in $[71,70]$ that $\sum_{i j} \omega_{i j} \otimes\left|\xi_{i}\right\rangle\left\langle\xi_{j}\right| \in M_{n} \otimes V^{*}$ defines a completely positive map if and only if

$$
\tilde{\omega}: \sum_{i j} v_{i j} \otimes\left|\xi_{i}\right\rangle\left\langle\xi_{j}\right| \mapsto \sum_{i j} \omega_{i j}\left(v_{i j}\right)
$$

defines a positive functional on $M_{n} \otimes V$, i.e. $\tilde{\omega}\left(C_{n}\right) \subset \mathbb{R}_{+}$. Conversely, if $\omega$ is a positive functional on $M_{n} \otimes V$, i.e. $\omega\left(C_{n}\right) \subset \mathbb{R}_{+}$, then the map

$$
\hat{\omega}: V \rightarrow M_{n}, \quad \hat{\omega}(v)=\sum_{i j} \omega\left(v \otimes\left|\xi_{i}\right\rangle\left\langle\xi_{j}\right|\right)\left|\xi_{i}\right\rangle\left\langle\xi_{j}\right|
$$

is completely positive. It follows from these results that $C_{n}^{*}$ defines a compatible family of cones for $M_{n} \otimes V^{*}$, which turns it into a *-matrix ordered space. If $V$ and hence $V^{*}$ are finite dimensional, then any element of the interior of $V_{+}{ }^{*}$ is an archimedian order unit, and it can be checked that in this case $V^{*}$ is also an operator system [15].

In the same way as positive elements of $M_{n} \otimes V^{*}$ can be identified with completely positive maps $\hat{\omega}: V \rightarrow M_{n}$, any element $v=\sum_{i j} v_{i j} \otimes\left|\xi_{i}\right\rangle\left\langle\xi_{j}\right| \in M_{n} \otimes V$ defines a completely positive map $\hat{v}: V^{*} \rightarrow M_{n}$ by setting

$$
\hat{v}(\omega)=\sum_{i j} \omega\left(v_{i j}\right)\left|\xi_{i}\right\rangle\left\langle\xi_{j}\right|
$$

We call a completely positive map $T: V^{*} \rightarrow M_{k}$ trace preserving, if $\operatorname{Tr} T(f)=f(e)$. We find that for $v=\sum_{i j} v_{i j} \otimes\left|\xi_{i}\right\rangle\left\langle\xi_{j}\right| \in M_{n} \otimes V$ the map $\hat{v}$ is trace preserving if the sum of the diagonal elements of $v$ is the identity in $V, \sum_{i} v_{i i}=e$.

Example 3.2.1. Fix $k \in \mathbb{N}$ and let $V \subset M_{k}$ be a linear subspace of the $\mathrm{C}^{*}$-algebra of $k \times k$ complex matrices, containing the identity $\mathbb{I}_{k}$. We denote the set of positive matrices again by $\left(M_{k}\right)_{+}$and set $V_{+}=V \cap\left(M_{k}\right)_{+}$. As shown in example 3.1.2, this turns the triple $\left(V, V_{+}, \mathbb{I}_{k}\right)$ into an $*$-AOU space. There is a natural operator system structure associated with it, namely the cones

$$
v \in C_{n} \subset M_{n} \otimes M_{k} \cong M_{n k} \Leftrightarrow v \in\left(M_{n k}\right)_{+} .
$$

It is easily checked that $\mathbb{1}_{n k}$ is an archimedian order unit for $C_{n}$, turning $V$ into an operator system. Then the duality between completely positive maps and positive functionals on the 
joint system is well-known and often called the Choi-Jamiolkowski isomorphism. Indeed, this duality was considered in the case of general operator systems by Choi and Effros, [15], and discussed in the case of matrix algebras by de Pillis and Jamiolkowski [19, 43].

We consider another physical example of an operator system. It serves as a starting point in our study of Tsirelson's problem.

Example 3.2.2. Consider the setting of a single system, where some measurements are performed. As described earlier, it follows from general considerations that this situation can be modeled by some $*$-AOU space $\left(V, V_{+}, e\right)$. A measurement is then described by a collection of effects $a_{\alpha}$, labeled by the outcome $\alpha$. Since we want that all outcomes are recorded, we require that $\sum_{\alpha} a_{\alpha}=e_{A}$. Consider an arbitrary quantum system described by some system Hilbert space $\mathcal{H}$. A measurement device is then a collection of positive operators $X_{\alpha}$ labeled by the outcomes $\alpha$ which sum up to the identity, $\sum_{\alpha} X_{\alpha}=\mathbb{I}$. Hence, a choice of a Hilbert space $\mathcal{H}$ and quantum observables with the given outcomes is a valid quantum representation of $\left(V, V_{+}, e\right)$, if it is a linear map $T: V \rightarrow \mathcal{B}(\mathcal{H})$ which is positive $T\left(V_{+}\right) \subset \mathcal{B}(\mathcal{H})_{+}$and unital, $T(e)=\mathbb{I}_{\mathcal{H}}$. Clearly, this will lead to observables $X_{\alpha}=T\left(a_{\alpha}\right)$. However, this is in general not physically sufficient, since we want that this representation is stable under the joining of $V$ (or its image under $T$ ) with some finite-dimensional quantum system. This leads to the requirement that $T$ is completely positive,

$$
\operatorname{id}_{n} \otimes T(x) \in \mathcal{B}\left(\mathbb{C}^{n} \otimes \mathcal{H}\right)
$$

for all $x \in\left(M_{n} \otimes V\right)_{+}$, where we assumed that the notion of joining the physical system $V$ with a finite-dimensional quantum system is uniquely defined, i.e. $V$ can be turned into an operator system. Thus, for each map $T$ we can think of its image as a self-adjoint linear subspace of $\mathcal{B}(\mathcal{H})$. On any such subspace $S$ the inclusion

$$
M_{n} \otimes S \subset \mathcal{B}\left(\mathbb{C}^{n} \otimes \mathcal{H}\right)
$$

defines a canonical matrix ordering, which is easily checked to satisfy the requirements of matrix cones. In particular, if $\mathbb{I}_{\mathcal{H}} \in S$, then it is an archimedian order unit for the matrix cones. Such a subspace $S \subset \mathcal{B}(\mathcal{H})$, being self-adjoint and containing the identity, is called a concrete operator system.

Representation theorem. Concrete operator systems are prime examples of general operator systems. In fact, it follows from the next theorem that any general operator system can be realized as a concrete one. This is the analog of Kadison's characterization of real archimedian order unit vector spaces and its generalization to the complex case, Theorem 3.1 .3 , to the category of operator systems.

Theorem 3.2.1 (Choi and Effros, [15]). Let $\left(V,\left(M_{n} \otimes V\right)_{+}, e\right)$ be an operator system. Then there exists a Hilbert space $\mathcal{H}$ and a completely positive unital map $T: V \rightarrow \mathcal{B}(\mathcal{H})$ which is an order isomorphism onto its range.

The proof is in some way analogous to the Kadison construction. Instead of using states, we use the corresponding notion for operator systems, that is, completely positive maps into matrix algebras. We introduce the index set

$$
\mathcal{I} \equiv\left\{\phi \mid \phi: V \rightarrow M_{n} \text { completely positive and unital }\right\},
$$


and define the map

$$
\begin{aligned}
& \Phi: V \rightarrow \bigoplus_{\phi \in \mathcal{I}} M_{n}(\phi) \\
& \Phi: x \mapsto \bigoplus_{\phi \in \mathcal{I}} \phi(x)
\end{aligned}
$$

where $M_{n}(\phi)$ denotes the image space of some element of $\mathcal{I}$. The range of $\Phi$ lies in a direct sum of $\mathrm{C}^{*}$-algebras. This direct sum is itself a $\mathrm{C}^{*}$-algebra, denoted by $\mathcal{A}$, and we may represent it faithfully on some Hilbert space $\mathcal{H}$. Hence it is enough to prove that $\Phi$ is a complete order embedding, or equivalently, the operator systems $V$ and $\Phi(V)$ are completely order isomorphic. This requires that for elements $x \in M_{n} \otimes V$ we have $x \geq 0$ if and only if $\Phi(v) \geq 0$, where the later positivity condition is checked with respect to the canonical ordering on $M_{n} \otimes \mathcal{A}^{7}$. The if part is easily seen, since all maps $\phi \in \mathcal{I}$ are assumed to be completely positive. For the converse, let $x \in M_{n} \otimes V$ but $x \nsupseteq 0$. Since the matrix cone $\left(M_{n} \otimes V\right)_{+}$is closed in the order topology, by the Krein-Milman theorem there exists a bounded functional $\omega$ on $M_{n} \otimes V$ which is positive on the cone and satisfies $\omega(x)<0$. But this implies, by the duality between positive functionals on the matrix cone $\left(M_{n} \otimes V\right)_{+}$and completely positive maps on $V$, that there exists a completely positive map $\phi_{\omega}: V \rightarrow M_{n}$ such that $\phi_{\omega}(x) \nsupseteq 0$. That is, there exists a vector $|\xi\rangle \in \mathbb{C}^{n}$ such that $\left\langle\xi \mid \phi_{\omega}(x) \xi\right\rangle<0$. It remains to construct a completely positive and unital map which achieves the same. This is a standard procedure and can be done in various ways, see for example the book by Paulsen [70]. We choose to apply Lemma 2.2 in the paper by Choi and Effros [15] which states that whenever $\phi: V \rightarrow \mathcal{N}$ is a completely positive map into a von Neumann algebra, then there is a completely positive and unital map $\psi: V \rightarrow \mathcal{N}$ such that $\phi(x)=b^{\frac{1}{2}} \psi(x) b^{\frac{1}{2}}$, where $b=\phi\left(\mathbb{I}_{V}\right)$. Hence, applying the lemma to $\phi_{\omega}$ and setting $|\tilde{\xi}\rangle=b^{\frac{1}{2}}|\xi\rangle$ we end up with a completely positive unital map $\psi_{\omega}: V \rightarrow M_{n}$ such that

$$
\left\langle\tilde{\xi} \mid \psi_{\omega}(x) \tilde{\xi}\right\rangle=\left\langle\xi \mid \phi_{\omega}(x) \xi\right\rangle<0,
$$

and hence $x \notin\left(M_{n} \otimes V\right)_{+}$. It follows that $\Phi$ is a complete order embedding. The lemma of Choi and Effros is used frequently in this thesis, and we call it the unitization trick.

Hence any operator system can be embedded completely order isomorphically into some $\mathcal{B}(\mathcal{H})$, for some Hilbert space $\mathcal{H}$. It also follows along the lines of the above reasoning, that in checking whether an element $x \in M_{n} \otimes V$ of some operator system is positive, it is sufficient to check positivity with respect to the set completely positive maps into matrix algebras. That is, only completely positive maps onto finite-dimensional $\mathrm{C}^{*}$-algebras are necessary in checking the positivity conditions of operator systems. For a proof, see for example Lemma 4.1 in [51].

Order norms. Every operator system $\left(V,\left\{C_{n}\right\}, e\right)$ can be equipped with a norm compatible to the order by setting

$$
\|v\|_{M_{n} \otimes V}=\inf \left\{\lambda>0:\left(\begin{array}{c}
\lambda e v \\
v^{*} \lambda e
\end{array}\right) \in C_{2 n}\right\}
$$

\footnotetext{
${ }^{7}$ Note that $M_{n}$ is a nuclear $\mathrm{C}^{*}$-algebra and hence its tensor product with any other $\mathrm{C}^{*}$-algebra is uniquely defined.
} 
for $v \in M_{n} \otimes V$. This turns the space $V$ into an operator space, which is loosely speaking the analog of operator system in the category of Banach spaces. That is, a Banach space $E$ is called an operator space, if there exists a sequence of norms on the Banach spaces $M_{n} \otimes E$, which fulfill certain consistency conditions analogous to the consistency conditions of matrix cones for some operator system. The analog of the Choi-Effros representation theorem for operator spaces was proved by Ruan [79], and gives rise to a completely bounded embedding of some abstract operator space into $\mathcal{B}(\mathcal{H})$, for some Hilbert space $\mathcal{H}$. The theory of operator spaces, also started later, is much further developed than the theory of operator systems. See for example the extensive book by Pisier, [74].

Quotients of operator systems. We now turn to summarize the notions of quotients of tensor products in the operator system category. Almost all of the material is taken from the excellent papers by Kavruk, Paulsen, Todorov and Tomforde [71, 52]. We start by considering quotients. Let $V$ be an operator system and $J \subset V$ a subspace. We would like to turn the algebraic quotient $V / J$ into an operator system. The first obvious requirement is that $\mathbb{I}_{V} \notin J$, i.e. the subspace should not contain the identity. From the theory of ordered vector spaces, we know that if $J$ is an order ideal, i.e. we have the implication

$$
a \in V, b \in J \quad 0 \leq a \leq b \Rightarrow a \in J,
$$

then the algebraic quotient $V / J$ can be equipped with an ordering given by the cone $V_{+}+J$ turning it into an ordered unit vector space. The element $e_{V}+J$ is then an order unit for the algebraic space $V / J$. However, it does not have to be an archimedian order unit.

Physically, this can be explained again by the fact that on the one hand the resulting cone does not have to be closed, on the other hand their might still be elements in $V / J$ which cannot be distinguished by the states of the system. This deficit in the category of $*$-AOU spaces was overcome by Paulsen and Tomforde, see [72], by applying their Archimedization process. It can also be described as a single quotient.

Let $\left(V, V_{+}, e_{V}\right)$ be an $*$-AOU space, and let $J \subset V$ be an order ideal. As indicated, we have to suitably enlarge the order ideal $J$. This is done by setting

$$
N_{J, h} \equiv\left\{v \in V_{h} \mid \forall \varepsilon>0 \text { exists } j, k \in J: \varepsilon e_{V}+j+v \in V_{+} \text {and } \varepsilon e_{V}+k-v \in V_{+}\right\},
$$

where $V_{h}$ denotes the hermitian subspace of $V$. If we set $N_{J} \equiv N_{J, h} \oplus i N_{J, h}$, then trivially, $J \subset N_{J}$ and by definition $N_{J}$ is closed under the involution. The involution on the algebraic quotient $V / N_{J}$ is defined canonically. The cone on the algebraic vector space $V / N_{J}$ is defined as

$$
\left(V / N_{J}\right)_{+} \equiv\left\{v+J \mid \forall \varepsilon>0 \text { exists } j \in N_{J}: \varepsilon e_{V}+v+j \in V_{+}\right\},
$$

and it can be proven that the order unit $e+J$ is an archimedian order unit for the cone $\left(V / N_{J}\right)_{+}$. It also follows that the associated algebraic quotient map $q: V \rightarrow V / J$ is positive and an order isomorphism between $V$ and $V / J \simeq V / \operatorname{ker} q$.

Alternatively, given another $*$-AOU space $\left(A, A_{+}, e_{A}\right)$ and some general unital positive map $\phi: V \rightarrow A$, then ker $\phi$ is an order ideal in $V$. Moreover, we have that $N_{\text {ker } \phi} \simeq \operatorname{ker} \phi$, that is, the enlarging of the order ideal ker $\phi$ is not necessary. It follows that the algebraic vector space $V / \operatorname{ker} \phi$ can be turned into a $*$-AOU space by defining the cone as

$$
(V / \operatorname{ker} \phi)_{+} \equiv\left\{v+J \mid \forall \varepsilon>0 \text { exists an } j \in \operatorname{ker} \phi: \varepsilon e_{V}+v+j \in V_{+}\right\} .
$$


The map $\tilde{\phi}: V / \operatorname{ker} \phi \rightarrow A$ defined by $\tilde{\phi}(x+\operatorname{ker} \phi)=\phi(x) \in A$ is positive, and if moreover $\phi\left(V_{+}\right) \simeq A_{+} \cap \operatorname{im} \phi$, then $\phi$ is also an order isomorphism onto its range.

In applying the above reasoning to operator systems, one comes to the conclusion that not every order ideal in some operator system is the kernel of a completely positive map. But if so, then the algebraic quotient can be turned into an $*$-AOU space, and the obvious idea is to extend this to all matrix levels. Following Paulsen and co-workers, we hence restrict ourselves to non-unital self-adjoint subspaces which are also kernels of completely positive maps.

Definition 3.2.3 (Kavruk et.al., [52]). Let $\left(V,\left(M_{n} \otimes V\right)_{+}, e\right)$ be an operator system, and let $J \subset V$ be a subspace. Then $J$ is called a kernel, if one of the following conditions holds.

(i) There exists an operator system $S$ and a completely positive unital map $\phi: V \rightarrow S$ such that $J \simeq \operatorname{ker} \phi$.

(ii) There exists an index set $\mathcal{I}$, operator systems $S_{\alpha}, \alpha \in \mathcal{I}$ and a collection of completely positive maps $\phi_{\alpha}: V \rightarrow S_{\alpha}$ such that $J \simeq \bigcap_{\alpha} \operatorname{ker} \phi_{\alpha}$.

(iii) There exists an index set $\mathcal{I}$, a collections of states $\omega_{\alpha}, \alpha \in \mathcal{I}$, such that $J \simeq$ $\bigcap_{\alpha \in \mathcal{I}} \operatorname{ker} \omega_{\alpha}$.

The following Proposition defining the quotient of operator systems is a corollary of the fact that operator systems are $*$-AOU spaces on every matrix level.

Proposition 3.2.2 (Kavruk et.al., [52]). Let $\left(V,\left(M_{n} \otimes V\right)_{+}, e\right)$ be an operator system, and let $J \subset V$ be a kernel. Then the algebraic quotient $V / J$, together with the set of matrix cones

$$
\left(M_{n} \otimes V / J\right)_{+} \equiv\left\{v+J \in M_{n} \otimes V \mid \forall \varepsilon>0 \text { exists an } j \in M_{n} \otimes J: \varepsilon e_{V}+v+j \in\left(M_{n} \otimes V\right)_{+}\right\}
$$

is a $*$-matrix ordered vector space and for every $n \in \mathbb{N}, \mathbb{I}_{n} \otimes(e+J)$ is an archimedian order unit. Hence $V / J$ is an operator system.

If $\phi: V \rightarrow S$ is a completely positive unital map between two operator systems, then ker $\phi$ is by definition a kernel in $V$. In correspondence to the case of $*$-AOU spaces, there exists a completely positive map $\tilde{\phi}: V / \operatorname{ker} \phi \rightarrow S$ such that $\phi=\tilde{\phi} \circ q$, where $q: V \rightarrow V / \operatorname{ker} \phi$ is the quotient map. By the first isomorphism theorem for completely positive maps, see [52], the map $\tilde{\phi}$ is moreover uniquely defined. If $\operatorname{id}_{n} \otimes \phi\left(M_{n} \otimes V\right)_{+}=\left(M_{n} \otimes S\right)_{+} \cap \operatorname{im}\left(\operatorname{id}_{n} \otimes \phi\right)$, then $\tilde{\phi}$ is a complete order isomorphism onto its range. In such a case the map $\phi$ is called a quotient map.

We call a kernel completely order proximinal, if, loosely speaking, the closure is not necessary, that is if we have

$$
\left(M_{n} \otimes V / J\right)_{+}=\left\{v+J \in M_{n} \otimes V \mid \text { there exists } j \in M_{n} \otimes J: v+j \in\left(M_{n} \otimes V\right)_{+}\right\} .
$$

It follows that if $J$ is a kernel in a finite-dimensional operator system, then $J$ is completely order proximinal. Likewise, it can be shown that if $\mathcal{A}$ is a $\mathrm{C}^{*}$-algebra and $J \subset \mathcal{A}$ is a closed two-sided ideal in $\mathcal{A}$, then $J$ is the kernel of the canonical $*$-homomorphism $q: \mathcal{A} \rightarrow \mathcal{A} / J$ (which is completely positive and unital) and moreover, $J$ is completely order proximinal. Within this thesis, if we speak of an ideal in a $\mathrm{C}^{*}$-algebra, then we always mean a closed, two-sided ideal. 
Tensor products in the category of operator systems. Throughout this paragraph, let $V$ and $S$ be operator systems, where we from now omit the mentioning of the matrix cones. Like in the case of $*$-AOU spaces, their algebraic tensor product

$$
S \otimes V
$$

can be turned into a vector space with an involution by setting $(a \otimes b)^{*} \equiv a^{*} \otimes b^{*}$. In order to turn it into an operator system, we have to specify matrix cones $C_{n}(S, V) \subset M_{n} \otimes S \otimes V$ such that the element $e_{S} \otimes e_{V}$ is an archimedian order unit. As in the case of $*$-AOU spaces, we require certain consistency conditions.

(i) The element $\mathbb{I}_{n} \otimes e_{S} \otimes e_{V}$ is an archimedian order unit for the cones $C_{n}(S, V)$.

(ii) If $a \in\left(M_{n} \otimes S\right)_{+}$and $b \in\left(M_{m} \otimes V\right)_{+}$, then $a \otimes b \in C_{n m}(S, V)$.

(iii) If $\phi: S \rightarrow M_{k}$ and $\psi: V \rightarrow M_{m}$ are completely positive maps, then $\operatorname{id}_{n} \otimes \phi \otimes$ $\psi\left(C_{n}(S, V)\right) \subset M_{n m k,+}$, that is $\phi \otimes \psi$ is also completely positive.

If a compatible family of matrix cones $C_{n}(S, V)$ satisfies these conditions, then it is called a set of matricial tensor product cones. Note that for the last item, we only considered completely positive maps into matrix algebras. If we moreover can relax this condition to general operator systems, then the system of cones is called functorial, [51]:

(iii') If $R$ and $T$ are any other operator systems, and $\phi: S \rightarrow R$ and $\psi: V \rightarrow T$ are completely positive maps, then $\operatorname{id}_{n} \otimes \phi \otimes \psi\left(C_{n}(S, V)\right) \subset C_{n}(R, T)$.

In analogy to the case of $*$-AOU spaces, there are two obvious choices for different matricial tensor product cones. The corresponding minimal tensor product is again defined with respect to elementary tensors of dual maps, which are now completely positive unital maps. The corresponding maximal tensor product is again defined using a suitable notion of jointly positivity.

Definition 3.2.4 (Kavruk et.al., [51]). Let $S$ and $V$ be operator systems and let $S \otimes$ $V$ be their algebraic tensor product. We denote by $C P_{u}\left(S, M_{n}\right)$ the set of completely positive maps from $S$ into some matrix algebra. The minimal matricial tensor product cone $C_{\min }(S, V)$ is the given by the family of matrix cones

$$
\begin{aligned}
& C_{n, \min }(S, V) \equiv\left\{z \in M_{n} \otimes S \otimes V \mid \phi \otimes \psi(z) \in M_{n m k,+}\right. \\
& \text { for all pairs } \left.(\phi, \psi) \in C P_{u}\left(S, M_{m}\right) \times C P_{u}\left(V, M_{k}\right)\right\} \text {. }
\end{aligned}
$$

We call the resulting operator system $\left(S \otimes V, C_{n, \min }(S, V), e_{S} \otimes V\right)$ the minimal tensor product of $S$ and $V$, and denote it by $S \otimes_{\min } V$. Let $\mathcal{H}$ be some Hilbert space and let $\tilde{\Omega}: S \times V \rightarrow \mathcal{B}(\mathcal{H})$ be a bilinear map. Then its canonical linear extension $\Omega$ to the algebraic tensor product $S \otimes V$ is called jointly completely positive, if we have that

$$
\Omega(a, b) \in \mathcal{B}\left(\mathbb{C}^{n m} \otimes \mathcal{H}\right)_{+} \text {for all elements } a \in M_{n} \otimes S \text { and } b \in M_{m} \otimes S .
$$

The maximal matricial tensor product cone is defined as

$$
\begin{aligned}
& C_{n, \max }(S, V) \equiv\left\{z \in M_{n} \otimes S \otimes V \mid \Omega(z) \in \mathcal{B}\left(\mathbb{C}^{n} \otimes \mathcal{H}\right)_{+}\right. \\
& \text {for all jointly completely positive maps } \Omega: S \otimes V \rightarrow \mathcal{B}(\mathcal{H})\} \text {. }
\end{aligned}
$$

We call the resulting operator system $\left(S \otimes V, C_{n, \max }(S, V), e_{S} \otimes V\right)$ the maximal tensor product of $S$ and $V$, and denote it by $S \otimes_{\max } V$. 
A proof that these definitions indeed satisfy the requirements of matricial tensor product cones can be found in the paper by Kavruk et. al., [51]. Like in the case of $*$-AOU spaces, the maximal tensor product can also be described intrinsically by the elementary cones.

Proposition 3.2.3 (Kavruk et.al., [51]). Let $S$ and $V$ be operator systems. If we define the (open) cones

$$
\begin{aligned}
D_{n, \max } \equiv\left\{\begin{array}{l}
z \\
z \in M_{n} \otimes S \otimes V \mid z=
\end{array}\right. & v^{*} a \otimes b v \text { for some pair } \\
& \left.(a, b) \in\left(M_{m} \otimes S\right)_{+} \times\left(M_{k} \otimes V\right)_{+} \text {and some linear map } v: \mathbb{C}^{n} \rightarrow \mathbb{C}^{m k}\right\}
\end{aligned}
$$

then the maximal matricial tensor product cones are the corresponding closure,

$$
C_{n, \max }=\left\{z \in M_{n} \otimes S \otimes V \mid \forall \varepsilon>0: \varepsilon e_{S} \otimes e_{V}+z \in D_{n, \max }\right\} .
$$

In the category of $*$-AOU spaces, the maximal tensor product cone was defined as the closure of the convex hull of the single positive cones. The matricial maximal tensor product cone is the natural generalization to operator systems, with the notion of the convex hull replaced by the "matrix convex hull", defined using the elementary matrix operations. This notion of "matrix convexity" was also studied independently from tensor products, see for example $[88,24,26]$.

The theory of tensor products of operator systems is inspired from the corresponding theory for *-AOU spaces, which in turn is based on the ideas developed by Alexandre Grothendieck [33] in the setting of Banach spaces. His theory of tensor products of Banach spaces, their geometry and their corresponding maps lead to the insights that approximation properties and tensor products are closely related. For further details, we refer the reader to the excellent book by Diestel and Uhl on vector measures on Banach spaces, [20]. We note that the theory of tensor products of Banach spaces also turned out to be useful in the study of Bell-like correlation tests, as touched in the previous section, see for example the authors diploma thesis [81] or the work of Marius Junge, Carlos Palazuelos and co-workers, see $[46,48,47]$, where the theory of tensor products of operator spaces was used.

Like in the case of operator systems, Grothendieck defined a minimal and maximal tensor product in the category of Banach spaces, but he called them the injective resp. the projective tensor product, due to their categorical properties. These properties, that is the behavior under embeddings or quotients, do carry over to the category of operator systems. We summarize the results in the following proposition.

Proposition 3.2.4 (Kavruk et.al., [51], Paulsen and Farenick, [27]). Let $S, V, R$ and $T$ be operator systems. If $\phi: S \rightarrow R$ and $\psi: V \rightarrow T$ is a pair of complete order embeddings, then its tensor product with respect to the minimal tensor product is again a complete order embedding. That is, if $S \subset R, V \subset T$ it follows that

$$
S \otimes_{\min } V \subset R \otimes_{\min } T .
$$

Hence the minimal tensor product is injective. Conversely, if $q: S \rightarrow R$ and $p: V \rightarrow T$ is a pair of quotient maps, their tensor product with respect to the maximal tensor product

$$
q \otimes p: S \otimes_{\max } V \rightarrow R \otimes_{\max } T
$$

is again a quotient map. Hence the maximal tensor product is projective. 
The names minimal and maximal tensor product can also be precisely understood. Namely, if $C_{n, \otimes}(S, V)$ is any family of compatible matricial tensor product cones on the algebraic tensor product $S \otimes V$ of two operator systems, then, [51]

$$
C_{n, \max }(S, V) \subset C_{n, \otimes}(S, V) \subset C_{n, \min }(S, V) .
$$

Hence, like in the case of $*$-AOU spaces, the minimal tensor product cone is the largest possible tensor product cone, and the maximal tensor product cone is the smallest possible. However, in the case their completely positive maps, the relation is reversed.

The injective nature of the minimal tensor product gives rise to another equivalent definition. If $S$ and $V$ are two operator systems, we can embed each of them completely order isomorphically into the $\mathrm{C}^{*}$-algebra of bounded operators on some Hilbert space. Let $\mathcal{H}$ and $\mathcal{K}$ be Hilbert spaces such that $S \subset \mathcal{B}(\mathcal{H}), V \subset \mathcal{B}(\mathcal{K})$. The minimal tensor product is given by the inclusion

$$
S \otimes_{\min } V \subset \mathcal{B}(\mathcal{H} \otimes \mathcal{K}) .
$$

For a proof, see [51]. It is based on the fact that the minimal $\mathrm{C}^{*}$-tensor product of $\mathcal{B}(\mathcal{H})$ with $\mathcal{B}(\mathcal{K})$ is equal to $\mathcal{B}(\mathcal{H} \otimes \mathcal{K})$. It follows from this inclusion that if $S$ and $V$ are in fact $\mathrm{C}^{*}$-algebras, then their minimal tensor product in the category of $\mathrm{C}^{*}$-algebras and their minimal tensor product in the category of operator systems in fact coincide. Hence it is natural to ask what happens to the maximal $\mathrm{C}^{*}$-tensor product in category of operator systems.

For that, let us now consider the case when the operator systems $S$ and $V$ are contained in some $\mathrm{C}^{*}$-algebras, $S \subset \mathcal{A}, V \subset \mathcal{B}$. As explained above, this is the case for every operator system. The maximal tensor product of $\mathcal{A}$ with $\mathcal{B}$ in the category of $\mathrm{C}^{*}$-algebras is defined by taking all pairs of $*$-homomorphisms with commuting ranges into some $\mathcal{B}(\mathcal{H})$. Hence there is a third canonical matricial tensor product cone over the algebraic tensor product $S \otimes V \subset \mathcal{A} \otimes \mathcal{B}$, defined in analogy to the maximal $\mathrm{C}^{*}$-tensor product.

Definition 3.2.5 (Kavruk et.al., [51]). Let $S$ and $V$ be operator systems and let $S \otimes V$ be their algebraic tensor product. We denote by $C P_{u}(S, \mathcal{B}(\mathcal{H}))$ the set of completely positive maps from $S$ into the $\mathrm{C}^{*}$-algebra of bounded operators on some Hilbert space $\mathcal{H}$. If $\phi \in C P_{u}(S, \mathcal{B}(\mathcal{H}))$ and $\psi \in C P_{u}(V, \mathcal{B}(\mathcal{H}))$ are a pair of completely positive unital maps, then we say that $\phi$ and $\psi$ have commuting ranges, if for all $a \in S$ and $b \in V,[\phi(a), \psi(b)]=$ $\phi(a) \psi(b)=\psi(b) \phi(a)=0$. The commuting matricial tensor product cone $C_{c}(S, V)$ is given by the family of matrix cones

$$
\begin{aligned}
C_{n, c}(S, V) & \equiv\left\{z \in M_{n} \otimes S \otimes V \mid \phi \cdot \psi(z) \in \mathcal{B}\left(\mathbb{C}^{n} \otimes \mathcal{H}\right)_{+}\right. \\
& \text {for all pairs } \left.(\phi, \psi) \in C P_{u}(S, \mathcal{B}(\mathcal{H})) \times C P_{u}(V, \mathcal{B}(\mathcal{H})) \text { with commuting ranges }\right\} .
\end{aligned}
$$

We call the resulting operator system $\left(S \otimes V, C_{n, c}(S, V), e_{S} \otimes e_{V}\right)$ the commuting tensor product of $S$ and $V$, and denote it by $S \otimes_{c} V$.

The commuting tensor product is in general not equal to the maximal tensor product of two operator systems, the exception being the case that one of the operator systems is actually a $\mathrm{C}^{*}$-algebra. That is, if $\mathcal{A}$ is a $\mathrm{C}^{*}$-algebra and $S$ is an operator system, then

$$
S \otimes_{c} \mathcal{A} \simeq S \otimes_{\max } \mathcal{A} .
$$

For a proof of this statement, see [51]. 
Like in the case of $*$-AOU spaces, it is naturally to ask for the nuclear elements in the category of operator systems, that is operator systems such that the minimal and the maximal tensor product coincide for all tensor pairings with other operator systems. Since by definition every operator system has a unique tensor product with all finitedimensional matrix algebras, every matrix algebra is nuclear within the category of operator systems. And like in the case of $*$-AOU spaces, nuclearity translates into a notion of strong approximation by these model objects.

Theorem 3.2.5 (Han and Paulsen, [40]). Let $S$ be an operator system. Then the following are equivalent.

(i) $S$ is nuclear, that is we have

$$
S \otimes_{\min } V \simeq S \otimes_{\max } V
$$

for any other operator system $V$.

(ii) For any $\varepsilon>0$, there exists a matrix algebra $M_{n(\varepsilon)}$ and a pair of completely positive and unital maps $u_{\varepsilon}: S \rightarrow M_{n(\varepsilon)}, v_{\varepsilon}: M_{n(\varepsilon)} \rightarrow S$ such that the diagram

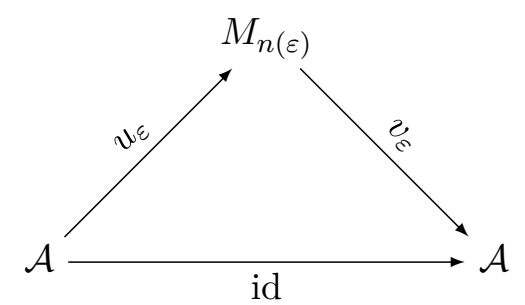

approximately commutes in the point-norm topology. That is we have

$$
\left\|v_{\varepsilon} \circ u_{\varepsilon}(x)-x\right\| \leq \varepsilon,
$$

where the norm is the canonical norm defined with respect to the matrix order on $S$.

It follows from this result that the same approximation it true for nuclear $\mathrm{C}^{*}$-algebras, which was actually known much earlier, due to work of Choi and Effros, [15].

It is hence natural to ask for equality of the commuting tensor product and the minimal tensor product. This is a central topic of this thesis, in particular if we ask for this equality to hold not for all, but a single operator system. This will be the operator system modeling a finite number of measurements with a finite number of outcomes on some arbitrary quantum system.

We next turn to a summary of the universal objects in the category of operator systems.

Universal objects of operator systems. For every operator system $S$, there exist three different universal $\mathrm{C}^{*}$-algebras, the universal $\mathrm{C}^{*}$-algebra $C^{*}(S)$, the injective envelope $I(S)$, and the universal enveloping $\mathrm{C}^{*}$-algebra $C_{e}^{*}(S)$. We introduce all of them to give a selfcontained overview.

The universal $\mathrm{C}^{*}$-algebra $C^{*}(S)$ can be constructed from the tensor algebra of some operator system $S$, that is from the free *-algebra

$$
\mathcal{T}(S) \equiv \bigoplus_{n} \bigotimes_{1}^{n} S
$$


where the multiplication is given by the tensor rule, $a \cdot b \equiv a \otimes b$, for $a, b$ in $S$. Given some completely positive unital map $\phi: S \rightarrow \mathcal{B}(\mathcal{H})$ into the bounded operators on some Hilbert space, it is easily seen that this map lifts to a $*$-representation $\pi_{\phi}$ of the tensor algebra by setting

$$
\pi_{\phi}(a \otimes \cdots \otimes b) \equiv \phi(a) \cdots \phi(b),
$$

and extending linearly. The universal $\mathrm{C}^{*}$-algebra $C^{*}(S)$ is then defined as the closure of the tensor algebra of $S$ with respect to the norm

$$
\|x\|_{C^{*}(S)} \equiv \sup _{\phi \in C P_{u}(S, \mathcal{B}(\mathcal{H}))}\left\|\pi_{\phi}(x)\right\|,
$$

and it is easily seen to have the following universal property. If $\mathcal{A}$ is some $\mathrm{C}^{*}$-algebra, and $\phi: S \rightarrow \mathcal{A}$ is a completely positive unital map, then there exists a $*$-homomorphism $\pi_{\phi}$ : $C^{*}(S) \rightarrow \mathcal{A}$, extending $\phi$. This can be used for another representation of the commuting tensor product of two operator systems. Since $S \subset C^{*}(S)$ completely order isomorphically, the maximal $\mathrm{C}^{*}$-tensor product between the universal $\mathrm{C}^{*}$-algebras $C^{*}(S)$ and $C^{*}(V)$ for another operator system $V$ gives rise to a tensor product by the inclusion, and this is the commuting tensor product, that is

$$
S \otimes_{c} V \subset C^{*}(S) \otimes_{\max } C^{*}(V) .
$$

For a proof, see [51].

In order to describe the next universal object, we first need to recall the notion of injective operator systems. Let $S$ be an operator system. Then $S$ is called injective, if for every pair of operator systems $V, W$ such that $V \subset W$ and every completely positive map $\phi: V \rightarrow S$, there exists an extension $\tilde{\phi}: W \rightarrow S$ such that the following diagram commutes.

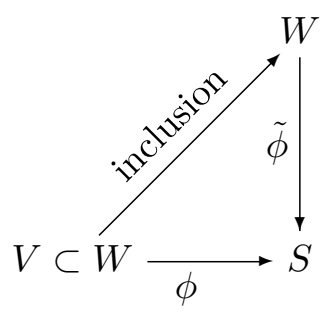

An extensively used theorem of Arveson states that for every Hilbert space $\mathcal{H}$, the operator system $\mathcal{B}(\mathcal{H})$ is injective [4]. Every injective operator system is also an injective $\mathrm{C}^{*}$-algebra, which can be seen by applying the definition to the inclusion $\iota: S \rightarrow \mathcal{B}(\mathcal{H})$, which lifts to a completely positive projection $P$ from $\mathcal{B}(\mathcal{H})$ onto $S$. Following Choi and Effros [15], we can define a product on $S$ by setting

$$
a \circ b \equiv P(\iota(a) \cdot \iota(b)) .
$$

See the book of Paulsen [70], chapter 15 for a proof that this product indeed gives rise to a $\mathrm{C}^{*}$-structure on $S$. The injective envelope $I(S)$ of a general operator system $S$ is the smallest injective operator system such that $S \subset I(S)$. We refer the reader to the book of Paulsen [70] for a proof of its existence. It is easily seen that this $\mathrm{C}^{*}$-algebra is injective as a $\mathrm{C}^{*}$-algebra, that is, there exists a faithful representation $I(S) \subset \mathcal{B}(\mathcal{H})$ such that there is a projection of norm one from $\mathcal{B}(\mathcal{H})$ onto $I(S)$. 
The universal enveloping $\mathrm{C}^{*}$-algebra $C_{e}^{*}(S)$ is the $\mathrm{C}^{*}$-algebra generated by $S$ inside its injective envelope. It has the following universal property. If $\mathcal{A}$ is a $\mathrm{C}^{*}$-algebra, and $\phi: S \rightarrow \mathcal{A}$ is a completely positive map, then there is a $*$-homomorphism $\pi: \mathcal{A} \rightarrow C_{e}^{*}(S)$ such that $\pi(\phi(x))=x$, for $x \in S$ where we see $S$ as embedded into $C_{e}^{*}(S)$.

The double commutant expectation and local lifting property for operator systems. Following the work of Kavruk, Paulsen, Todorov and Tomforde [52], we define extensions of the $\mathrm{C}^{*}$-algebraic weak expectation and local lifting properties for operator systems. Recall that a $\mathrm{C}^{*}$-algebra $\mathcal{A}$ has the weak expectation property, in short the WEP, if there exists a faithful inclusion $\mathcal{A} \subset \mathcal{A}^{* *} \subset \mathcal{B}(\mathcal{H})$, such that there is a completely positive map $E: \mathcal{B}(\mathcal{H}) \rightarrow \mathcal{A}^{* *}$ which on $\mathcal{A}$ reduces to the identity, $\left.E\right|_{\mathcal{A}}=\mathrm{id}_{\mathcal{A}}$. Such a map $E$ is called a weak expectation of $\mathcal{A}$ relative to $\mathcal{B}(\mathcal{H})$, and this notion has an obvious extension to inclusions $\mathcal{A} \subset \mathcal{B}$, where $\mathcal{B}$ is another $\mathrm{C}^{*}$-algebra ${ }^{8}$. We note that the bi-dual $\mathcal{A}^{* *}$ of a $\mathrm{C}^{*}$-algebra is always a von Neumann algebra. The weak expectation property can also be defined without referring to the double dual directly, but instead to the von Neumann algebra generated by a faithful representation of $\mathcal{A}$. We first recall the definition of commutants. If $\mathcal{G} \subset \mathcal{B}$ is a set in a $\mathrm{C}^{*}$-algebra, the commutant $\mathcal{G}^{\prime}$ of $\mathcal{G}$ in $\mathcal{B}$ is the set

$$
\mathcal{G}^{\prime}=\{b \in \mathcal{B} \mid[a, b]=0 \forall a \in \mathcal{G}\}
$$

which is easily seen to be a $\mathrm{C}^{*}$-algebra. The double commutant of a $\mathrm{C}^{*}$-algebra $\mathcal{A}, \mathcal{A}^{\prime \prime}=$ $\left(\mathcal{A}^{\prime}\right)^{\prime}$ computed with respect to some inclusion $\mathcal{A} \subset \mathcal{B}(\mathcal{H})$ is always a von Neumann algebra, and is called the von Neumann algebra generated by this inclusion. If the inclusion is faithful, this gives rise to another equivalent definition of the WEP. That is, $\mathcal{A}$ has the WEP if there is a faithful representation $\pi: \mathcal{A} \rightarrow \mathcal{B}(\mathcal{H})$ such that there is a completely positive map, into the double commutant of $\pi(\mathcal{A}), E: I(\mathcal{A}) \rightarrow \pi(\mathcal{A})^{\prime \prime}$, which fixes $\mathcal{A}$, i.e. $E(a)=a$ for $a \in \mathcal{A}$. Here, $I(\mathcal{A})$ is the injective envelope of $\mathcal{A}$, which existence we noted earlier. This suggest the following generalization to the category of operator systems.

Definition 3.2.6 (Kavruk et.al., [52]). Let $S$ be an operator system and let $I(S)$ be its injective envelope. Then we say that $S$ has the double commutant expectation property, in short DCEP, if for every complete order embedding $\iota: S \rightarrow \mathcal{B}(\mathcal{H})$, there exists a completely positive unital map $\varphi: I(S) \rightarrow \iota(S)^{\prime \prime}$, which fixes the operator system $S$, that is, $\varphi(s)=s$, where we identified $S$ first with its copy in $I(S)$ and second with $\iota(S) \subset \mathcal{B}(\mathcal{H})$. Here, the double commutant is calculated in $\mathcal{B}(\mathcal{H})$.

Like in $\mathrm{C}^{*}$-algebra case, the DCEP and tensor products are closely related. The following theorem is again due to the work of Kavruk, Paulsen, Todorov and Tomforde [52], and is the generalization of the corresponding $\mathrm{C}^{*}$-algebraic results, mainly obtained by Kirchberg.

Theorem 3.2.6 (Kavruk et.al., [52]). Let $S$ be an operator system. Then the following are equivalent.

(i) The operator system $S$ has the DCEP.

(ii) For every inclusion $S \subset \mathcal{B}(\mathcal{H})$ there exist a completely positive map $\Phi: \mathcal{B}(\mathcal{H}) \rightarrow S^{\prime \prime}$ which reduces to the identity on $S$. Here, the double commutant is computed in $\mathcal{B}(\mathcal{H})$.

\footnotetext{
${ }^{8}$ That is, a weak expectation $E$ of $\mathcal{A}$ relative to $\mathcal{B}$ is a map $E: \mathcal{B} \rightarrow \mathcal{A}^{* *}$ such that $\left.E\right|_{\mathcal{A}}=\operatorname{id}_{\mathcal{A}}$, for $\mathcal{A} \subset \mathcal{B}$.
} 
(iii) For any operator system $V$ with $S \subset V$ and any operator system $T$ we have

$$
S \otimes_{c} T \subset V \otimes_{c} T .
$$

(iv) For any operator system $V$ with $S \subset V$ and any $C^{*}$-algebra $\mathcal{B}$ we have

$$
S \otimes_{\max } \mathcal{B} \subset V \otimes_{\max } \mathcal{B} .
$$

(v) There exist an inclusion $S \subset \mathcal{B}(\mathcal{H})$ such that for any operator system

$$
S \otimes_{c} T \subset \mathcal{B}(\mathcal{H}) \otimes_{c} T .
$$

(vi) There exist an inclusion $S \subset \mathcal{B}(\mathcal{H})$ such that for all $n$

$$
S \otimes_{\max } C^{*}\left(\mathbb{F}_{n}\right) \subset \mathcal{B}(\mathcal{H}) \otimes_{\max } C^{*}\left(\mathbb{F}_{n}\right),
$$

where $C^{*}\left(\mathbb{F}_{n}\right)$ is the universal $C^{*}$-algebra of the free group with $n$ generators.

In chapter 5 of this thesis, we ask what happens if we replace the inclusion $S \subset \mathcal{B}(\mathcal{H})$ by an inclusion $S \subset \mathcal{B}$, where $\mathcal{B}$ is a $\mathrm{C}^{*}$-algebra which is QWEP. We find that this property, expressed using tensor products, again leads to a certain approximation properties.

We conclude this paragraph by introducing the operator system version of the local lifting property in the category of $\mathrm{C}^{*}$-algebra. A $\mathrm{C}^{*}$-algebra is said to have the local lifting property, in short LLP, if whenever $S \subset \mathcal{A}$ is a finite-dimensional subspace, and $\mathcal{B}$ is another $\mathrm{C}^{*}$-algebra with $J \subset \mathcal{B}$ being an ideal, there exists for any completely positive unital map $\phi: \mathcal{A} \rightarrow \mathcal{B} / J$ a completely bounded local lift $\tilde{\phi}: S \rightarrow \mathcal{B}$, such that $\left.\phi\right|_{S}=q \circ \tilde{\phi}$, where $q: \mathcal{B} \rightarrow \mathcal{B} / J$ is the quotient map.

The generalization to the category of operator system was introduced in [52].

Definition 3.2.7 (Kavruk et.al., [52]). Let $S$ be an operator system, $\mathcal{B}$ a $\mathrm{C}^{*}$-algebra, $J \subset \mathcal{B}$ a (closed, two-sided) ideal in $\mathcal{B}$ and let $q: \mathcal{B} \rightarrow \mathcal{B} / J$ be the quotient map. A completely positive unital map $\phi: S \rightarrow \mathcal{B} / J$ is said to lift locally, it there is for any finite-dimensional operator (sub)-system $V \subset S$ a completely positive map $\tilde{\phi}: V \rightarrow \mathcal{B}$ such that $q \circ \tilde{\phi}=\left.\phi\right|_{S}$. An operator system $S$ is said to have the operator system local lifting property, in short OSLLP, if for every pair of a $\mathrm{C}^{*}$-algebra $\mathcal{B}$ and an ideal $J \subset \mathcal{B}$, every completely positive $\operatorname{map} \phi: S \rightarrow \mathcal{B} / J$ lifts locally.

Like the DCEP, the OSLLP for operator systems can also be expressed using tensor products. The $\mathrm{C}^{*}$-algebraic analog of the next theorem is well-known and due to Kirchberg $[55]$.

Theorem 3.2.7 (Kavruk et.al., [52]). Let $S$ be an operator system. Then the following are equivalent.

(i) The operator system $S$ has the OSLLP.

(ii) For every Hilbert space $\mathcal{H}$, we have that $S \otimes_{\min } \mathcal{B}(\mathcal{H})=S \otimes_{\max } \mathcal{B}(\mathcal{H})$.

In the later parts of this thesis, we need the following tensorial property of a pair of operator systems one having the DCEP while the other one has the OSLLP. Its $\mathrm{C}^{*}$-algebraic analog is well-known, but we were unable to find a proof for operator systems. 
Lemma 3.2.8. Let $S$ and $T$ be operator systems. If $S$ has the DCEP, and $T$ the OSLLP, then the minimal and the commuting tensor product are completely isomorphic,

$$
S \otimes_{\min } T=S \otimes_{c} T .
$$

Proof. We know that $S \otimes_{c} T \subset \mathcal{B}(\mathcal{H}) \otimes_{c} T$. But $\mathcal{B}(\mathcal{H}) \otimes_{c} T=\mathcal{B}(\mathcal{H}) \otimes_{\max } T=\mathcal{B}(\mathcal{H}) \otimes_{\min } T$, since $T$ has the OSLLP. Hence we have the following chain of inclusions

$$
S \otimes_{c} T \subset \mathcal{B}(\mathcal{H}) \otimes_{c} T=\mathcal{B}(\mathcal{H}) \otimes_{\max } T=\mathcal{B}(\mathcal{H}) \otimes_{\min } T \supset S \otimes_{\min } T,
$$

from which the statement follows.

An operator system version of Kirchberg's QWEP conjecture. We close this chapter by stating an operator system version of Connes' embedding problem, first obtained by Farenick and Paulsen, and improved by Kavruk. We take $2 n$ universal unitaries $w_{i}, v_{k}$, such that $\left[u_{i}, v_{k}\right]=0$ for all $i, k \in\{1, \ldots, n\}$, and $u_{1}=v_{1}=\mathbb{I}_{\mathcal{H}}$. Being a universal set of $2 n$ unitaries, these elements generate the $\mathrm{C}^{*}$-algebra $C^{*}\left(\mathbb{F}_{n-1}\right) \otimes_{\max } C^{*}\left(\mathbb{F}_{n-1}\right)=C^{*}\left(\mathbb{F}_{n-1} \times \mathbb{F}_{n-1}\right)$. The operator system

$$
\mathcal{W}_{n} \equiv \operatorname{span}\left\{w_{i}^{*} w_{j}\right\} \subset C^{*}\left(\mathbb{F}_{n-1}\right)
$$

was defined and analyzed in detail by Farenick and Paulsen, [27]. Note that $\mathcal{W}_{n}$ generates $C^{*}\left(\mathbb{F}_{n-1}\right)$ (we set the first unitary to be equal to the identity, hence the difference in the numbering). They discovered that it is completely order isomorphic to the range of the completely positive unital map

$$
\Phi: M_{n} \rightarrow C^{*}\left(\mathbb{F}_{n}\right) \quad \Phi(|i\rangle\langle j|) \equiv w_{i}^{*} w_{j} .
$$

The kernel $J_{n}$ of $\Phi$ is easily seen to be the subspace of $M_{n}$ consisting of diagonal trace-zero matrices, and hence we obtain a completely positive injective map $\tilde{\Phi}: M_{n} / J_{n} \rightarrow C^{*}\left(\mathbb{F}_{n}\right)$. Farenick and Paulsen [27] then used Halmos dilation construction to conclude that the inverse of $\tilde{\Phi}$ is also completely positive, hence obtaining an order isomorphism between $\mathcal{W}_{n}$ and the operator system quotient $M_{n} / J_{n}$. They also obtained the following relation with Kirchberg's and hence Connes' conjecture. Shortly afterwards, it was improved by Kavruk.

Theorem 3.2.9 (Farenick \& Paulsen, Kavruk [27, 50]). Let $\mathcal{W}_{n}$ be the operator system just described. If we have for all $n$ that

$$
\mathcal{W}_{n} \otimes_{\min } \mathcal{W}_{n}=\mathcal{W}_{n} \otimes_{\max } \mathcal{W}_{n}
$$

then Kirchberg's conjecture has a positive answer. Moreover, Kirchberg's conjecture has an affirmative answer if and only if

$$
\mathcal{W}_{3} \otimes_{\min } \mathcal{W}_{3}=\mathcal{W}_{3} \otimes_{c} \mathcal{W}_{3} .
$$

In the next chapter, we introduce and study another operator system which also gives rise to a reformulation of Kirchberg's conjecture. Its definition is motivated by the physical situation of two independent parties performing one out of a finite number of possible measurements with a finite number of outcomes on a joint quantum system. 



\section{Tsirelson's problem and the QWEP conjecture}

On the notion of bipartite quantum systems. "Tsirelson's problem" was first noted by Navascues and Acin [66] and formulated in a sharp way by Tsirelson [87]. It originates in an old paper by Tsirelson [86], therefore its name. The issue is the notion of "subsystem", or the kind of independence postulated between two parties, as usual named Alice and Bob. Both of them hold a finite number of measurement devices, also often referred to as settings. We assume for simplicity that the set of outcomes is finite. We denote by $I$ resp. $J$ the finite device sets of Alice and Bob, and to each device we associate a finite outcome set $A_{i}, i \in I$ resp. $B_{j}, j \in J$.

We are interested in the case when Alice and Bob want to perform independent measurements, meaning that Bob's choice of a particular measuring device does not depend on Alice's choice. The basic question is now how to model this situation mathematically assuming the laws of quantum mechanics.

In that case, a measurement device is characterized by a set of positive (and hence bounded) operators (POVM) acting on some Hilbert space $\mathcal{H}$, each associated to a particular outcome, summing up to the identity. Let $\{A\}_{i, \alpha} \subset \mathcal{B}(\mathcal{H})$ denote the collection of positive operators on Alice's side, $i \in I$ identifying the measurement device and $\alpha \in A_{i}$ being the index characterizing a particular outcome. Conversely, let $\{B\}_{j, \beta} \subset \mathcal{B}(\mathcal{H}), j \in J$, $\beta \in B_{j}$ be the set of operators on Bob's side. Here, $\mathcal{B}(\mathcal{H})$ denotes the algebra of all bounded operators on $\mathcal{H}$. For simplicity reasons, we drop the dependence of $\alpha$ and $\beta$ on $i$ and $j$.

Each measurement device is mapped to a probability distribution by means of a state, i.e. a positive and normalized linear functional $\omega: \mathcal{B}(\mathcal{H}) \rightarrow \mathbb{C}$, defined on the bounded operators with the additional property that $\omega(\mathbb{I})=1$. In finite dimensions, every state can be described by a positive operator $\rho$ having trace one, usually called a density matrix, by means of the identification $\omega(A)=\operatorname{tr} \rho A$.

In the usual scenario in quantum information theory, we assume that each part of the system can be characterized by a Hilbert space $\mathcal{H}_{A}$ or $\mathcal{H}_{B}$, and the joint physical system is described by the Hilbert space tensor product $\mathcal{H}=\mathcal{H}_{A} \otimes \mathcal{H}_{B}$. Each measurement operator on Alice's side acts only on $\mathcal{H}_{A}$, and conversely for Bob. The physical state of the joint system is then described by means of a state $\omega$ on the bounded operators of $\mathcal{H}_{A} \otimes \mathcal{H}_{B}$, the 
Hilbert space tensor product of Alice's and Bob's Hilbert space. The probability $p(i, j \mid \alpha, \beta)$ that Alice uses measurement device $i$ and detects outcome $\alpha$ while Bob uses measurement device $j$ and detects outcome $\beta$ is given by the expression

$$
p(i, j \mid \alpha, \beta)=\omega\left(A_{i, \alpha} \otimes B_{j, \beta}\right) .
$$

The assumption that Alice's choice of a measurement device does not depend on Bob's choice also means that their measurements can both be performed simultaneously, without causing any disturbance. Put differently, this implies that all measurement operators on Alice's side have to commute with Bob's measurement operators. See the introduction on tensor products of $\mathrm{C}^{*}$-algebras for a more detailed explanation of this reasoning. Thus, the same situation can be modeled by using only one Hilbert space $\mathcal{H}$ and a state $\omega$ on $\mathcal{B}(\mathcal{H})$. Now, Alice and Bob both hold a finite set of positive operators $\{A\}_{i, \alpha} \subset \mathcal{B}(\mathcal{H})$, resp. $\{B\}_{j, \beta} \subset \mathcal{B}(\mathcal{H})$ with the requirement that $\left[A_{i, \alpha}, B_{j, \beta}\right]=0$ for all $i, j, \alpha, \beta$. Accordingly, the probability $p(i, j \mid \alpha, \beta)$ that Alice uses measurement device $i$ and detects outcome $\alpha$ while Bob uses measurement device $j$ and detects outcome $\beta$ is then

$$
p(i, j \mid \alpha, \beta)=\omega\left(A_{i, \alpha} \cdot B_{j, \beta}\right) .
$$

The obvious question is now whether both models produce the same set of possible correlation functions. The answer is inherently connected to Kirchberg's QWEP conjecture, as is shown in this chapter. We start by modeling the situation in the language of operator systems.

\subsection{The operator system of local measurements}

The archimedian ordered vector space of one measurement. We start by considering a physical system, and for convenience the observer ${ }^{1}$ is called Alice. As described in the section about archimedian ordered vector spaces, it is natural to model this situation by a $*$-AOU space $\left(A, A_{+}, e\right)$. A measurement with a finite number $k \in \mathbb{N}$ of outcomes is then described by a collection of $k$ positive elements $a_{\alpha} \in A_{+}, \alpha \in\{1, \ldots, k\}$, such that $\sum_{\alpha} a_{\alpha}=e$. This abstract picture can also be modeled more concretely, by introducing the *-AOU space $\left(\ell_{k}^{\infty}, \ell_{k,+}^{\infty}, 1\right)$. Here, $\ell_{k}^{\infty}$ is the vector space of complex valued $k$-tuples with entry-wise involution, and $\ell_{k,+}^{\infty}$ is the cone of all tuples with positive entries, and 1 is the $k$-tuple with all entries equal to one. For $\alpha$ as before, we denote by $e_{\alpha} \in \ell_{k,+}^{\infty}$ the element where all entries, except at position $\alpha$, are equal to zero, and equal to one otherwise. Clearly we have that $\sum e_{\alpha}=e$. It is easily checked that the map sending $e_{\alpha}$ to $a_{\alpha}$, the positive element implementing the outcome $\alpha$, extends to a linear, positive and unital map from $\ell_{k}^{\infty}$ to the $*$-AOU space $A$. Hence, every physical experiment, which consists of a measurement with a finite number of outcome can be thought of as a positive unital map from the $*$-AOU space $\left(\ell_{k}^{\infty}, \ell_{k,+}^{\infty}, 1\right)$.

We note that since $\left(\ell_{k}^{\infty}, \ell_{k,+}^{\infty}, 1\right)$ is a nuclear $*$-AOU space, its tensor product with matrix algebras, or finite-dimensional quantum systems, is uniquely defined, which turns $\left(\ell_{k}^{\infty}, \ell_{k,+}^{\infty}, 1\right)$ into an operator system.

\footnotetext{
${ }^{1}$ We note that the notion of an observer is introduced in a purely linguistic way, to simplify the discussion. Instead of saying "the physical system is measured and the outcome $\alpha$ is observed", we just write "Alice' measures outcome $\alpha$ ". We do not enter the philosophical discussion on whether the notion of an observer is needed for the formulation of quantum physics.
} 
A quantum representation of this situation is a positive unital map from $\ell_{k}^{\infty}$ into the operator system $\mathcal{B}(\mathcal{H})$ of bounded operators on some Hilbert space $\mathcal{H}$, which models the quantum system to be measured. That is, each element $e_{\alpha} \in \ell_{k,+}^{\infty}$ is identified with a positive operator $A_{\alpha} \in \mathcal{B}(\mathcal{H})_{+}$and we have that $\sum_{\alpha} A_{\alpha}=\mathbb{I}_{\mathcal{H}}$. Such a collection of operators is also called a positive operator valued measure ${ }^{2}$, in short POVM. We note that any such positive map is automatically completely positive, which can be seen directly from the fact that $\ell_{k}^{\infty}$ is a commutative $\mathrm{C}^{*}$-algebra, see [70].

More settings. We now turn to the situation where we again only have one physical system to be observed, but more than one possible measurement devices. That is, Alice can chooses one out of a finite number, say $n \in N$, measurement settings, labeled by $i \in\{1, \ldots, n\}$ and then observes the outcome. As first try, we might consider the $*$-AOU space

$$
\ell_{n}^{\infty} \otimes \ell_{k}^{\infty}
$$

to be a possible model for this situation, identifying the element corresponding to the outcome $\alpha$ of the measurement $i$ with $e_{i} \otimes e_{\alpha}$. Note that as both tensor factors are nuclear *-AOU spaces, the tensor product cone is uniquely defined. However, this space is "too large", since the corresponding "do nothing" measurement, the identity, is the element $1 \otimes 1$. This means that in order to obtain the "do nothing" measurement, we have to sum up not only all possible outcomes, but also all possible measurements. This is certainly not so in the physical situation we want to model, since there, just summing up the outcomes corresponding to one measurement leads to the corresponding "do nothing" measurement. Hence we would like to identify the two distinct elements

$$
e_{i} \otimes \sum_{\alpha} e_{\alpha} \text { and for } i \neq j e_{j} \otimes \sum_{\alpha} e_{\alpha}
$$

That is, we are looking for an equivalence relation on the $*$-AOU space $\left(\ell_{n}^{\infty} \otimes \ell_{k}^{\infty}, \ell_{n,+}^{\infty} \otimes\right.$ $\left.\ell_{k,+}^{\infty}, 1 \otimes 1\right)$ such that

$$
e_{i} \otimes \sum_{\alpha} e_{\alpha} \sim e_{j} \otimes \sum_{\alpha} e_{\alpha}
$$

for all $i, j \in\{1, \ldots, n\}$. From the theory of quotients of $*$-AOU spaces and operator systems described in the last chapter, we know that we have to look for an order ideal $\mathcal{Q} \subset \ell_{n}^{\infty} \otimes \ell_{k}^{\infty}$ such that

$$
e_{i} \otimes \sum_{\alpha} e_{\alpha}-e_{j} \otimes \sum_{\alpha} e_{\alpha} \in \mathcal{Q}
$$

for all $i, j \in\{1, \ldots, n\}$. Since $e_{i} \otimes \sum_{\alpha} e_{\alpha}-e_{j} \otimes \sum_{\alpha} e_{\alpha}=\left(e_{i}-e_{j}\right) \otimes \sum_{\alpha} e_{\alpha}$, we see that $\mathcal{Q}$ has to be the subspace of elements being equal to the identity on the second tensor factor and having elements such that the sum of them is equal to zero,

$$
\mathcal{Q}=\left\{a \otimes 1 \in \ell_{n}^{\infty} \otimes \ell_{k}^{\infty} \mid \sum_{i} a_{i}=0\right\}
$$

where $a_{i}$ denotes the $i$-th entry of the $n$-tuple $a$. It is easily seen that $\mathcal{Q}$ is an order ideal, since the only positive element is 0 . Hence our choice for modeling the situation of $n$

\footnotetext{
${ }^{2}$ In this simple case, the measure space is the set of all $k$-tuples and the $\sigma$-algebra is just the the corresponding powerset.
} 
measurements with $k$ outcomes each is the quotient order unit vector space

$$
\left(\ell_{n}^{\infty} \otimes \ell_{k}^{\infty} / \mathcal{Q}, \ell_{n,+}^{\infty} \otimes \ell_{k,+}^{\infty}+\mathcal{Q}, 1 \otimes 1+\mathcal{Q}\right)
$$

We note that it is a priori not clear that this space is also a $*$-AOU space, due to the obstructions in taking quotients of $*$-AOU spaces as explained in the last chapter. Another notable fact is that while the space $\ell_{n}^{\infty} \otimes \ell_{k}^{\infty}$ was nuclear in the category of $*$-AOU spaces, its quotient is certainly not, since otherwise there would be no observable difference between classical and quantum correlations. Hence in particular, the tensor product cone of

$$
\left(\ell_{n}^{\infty} \otimes \ell_{k}^{\infty} / \mathcal{Q}\right) \otimes M_{n}
$$

is not uniquely defined. Thus, for meaningful describing physical situations where the second system is a finite-dimensional quantum system, a tensor product cone has to be defined. As explained in the last chapter, both deficits can be removed by defining an operator system structure on $\ell_{n}^{\infty} \otimes \ell_{k}^{\infty} / \mathcal{Q}$. Fortunately, Kavruk in [50] already studied quotients of operator systems by subspaces which contain 0 as the only possible positive element. Proposition 2.4 in [50] asserts us that $\mathcal{Q}$ is indeed a completely order proximinal kernel in the operator system sense. His result gives rise to the following definition.

Definition 4.1.1. Let $n, k$ be natural numbers. The operator system of $n$ local measurements with $k$ outcomes each, denoted by $\mathcal{L M}_{n, k}$, is the algebraic quotient $\ell_{n}^{\infty} \otimes \ell_{k}^{\infty} / \mathcal{Q}$ together with the set of matrix cones

$$
\begin{aligned}
\left(M_{n} \otimes \mathcal{L} \mathcal{M}_{n, k}\right)_{+}= & \left\{z \in M_{n} \otimes \mathcal{L} \mathcal{M}_{n, k} \mid\right. \\
& \text { there exists } \left.k \in M_{n} \otimes \mathcal{Q} \text { such that } z+k \in M_{n} \otimes \ell_{n,+}^{\infty} \otimes \ell_{k,+}^{\infty}\right\}
\end{aligned}
$$

and the archimedian order unit $1 \otimes 1+\mathcal{Q}$. Here, $M_{n} \otimes \ell_{n,+}^{\infty} \otimes \ell_{k,+}^{\infty}$ denotes the unique tensor product cone of the tensor product of the $*$-AOU spaces $M_{n}$ and $\ell_{n}^{\infty} \otimes \ell_{k}^{\infty}$.

Remark 4.1.1. This operator system was first constructed, albeit in a different way, in [46] and later in [45]. There, it was called the dual non-signaling operator system and denoted by $\operatorname{NSG}^{*}(N, K)$, for $N$ measurements with $K$ outcomes each. This first construction was inspired by operator space techniques, which lead to the same results. However, we think that the modeling of physical situations with $*$-AOU spaces is more intuitive, and therefore have chosen a presentation along these lines. Note that $\mathcal{L M}_{n, k}$ can be also constructed as the amalgamated direct sum of $n$ copies of the operator system $\ell_{k}^{\infty}$, also called the coproduct of $n$ copies of $\ell_{k}^{\infty}$. See the papers by Kavruk [50], section 8, and by Fritz [29], for these constructions for arbitrary operator systems.

Any physical situation where we want to model $n$ measurements with $k$ outcomes each is given by a completely positive unital $\phi$ map from $\ell_{n}^{\infty} \otimes \ell_{k}^{\infty}$ into the bounded operators on some Hilbert space $\mathcal{H}$, with the additional requirement that $\mathcal{Q} \subset \operatorname{ker} \phi$. This implies that there is a corresponding completely positive and unital map $\tilde{\phi}: \mathcal{L} \mathcal{M}_{n, k} \rightarrow \mathcal{B}(\mathcal{H})$, such that $\phi=\tilde{\phi} \circ q$, where $q: \ell_{n}^{\infty} \otimes \ell_{k}^{\infty} \rightarrow \mathcal{L} \mathcal{M}_{n, k}$ is the quotient map. Conversely, every completely positive and unital map $\phi: \mathcal{L M}_{n, k} \rightarrow \mathcal{B}(\mathcal{H})$ is a quantum representation of the situation just described. 
Free products of groups and $\mathbf{C}^{*}$-algebras. Another possibility to construct the operator system structure for $\ell_{n}^{\infty} \otimes \ell_{k}^{\infty} / \mathcal{Q}$ would be to find an operator system $S$ and a completely positive map $\phi$ from $\ell_{n}^{\infty} \otimes \ell_{k}^{\infty}$ (which due to its nuclearity is an operator system) into $S$, such that $\mathcal{Q}=\operatorname{ker} \phi$. As explained in the last chapter, this would allow us to construct matricial tensor product cones. In order to find such a pair of operator system and a corresponding completely positive unital map, we need to introduce the notion of free products of $\mathrm{C}^{*}$ algebras and their representation. This will also allows us to study the equivalence between Tsirelson's problem and the QWEP conjecture.

Given $n$ groups $G_{i}$, their free product $*_{n} G_{i}$ can be defined by the following universality property.

Definition 4.1.2. Let $H$ be any other group, and let $\phi_{i}: G_{i} \rightarrow H$ be a group homomorphism. The free product ${ }_{{ }_{n}} G_{i}$ of groups $G_{i}$ is the unique group containing the $G_{i}$ 's as subgroups, such that there is a unique homomorphism $\phi:{ }_{n} G_{i} \rightarrow H$, which reduces to $\phi_{i}$ if restricted to the subgroup $G_{i}$.

See any book on algebra for a proof of the existence of the free product and its uniqueness. A possible visualization of the free product is the set of all words with elements in one of the groups $G_{i}$, with no further relations than the identification of identity elements. Hence, unless one of the groups is trivial, the free product of two groups is always infinite. We note that if $\mathbb{F}_{n}$ is the free group of $n$ generators, than since there are no more relations in a free group, we have that $\mathbb{F}_{n} * \mathbb{F}_{m} \simeq \mathbb{F}_{n+m}$.

We turn to the analogous construction for $\mathrm{C}^{*}$-algebras. Instead of $n$ groups $G_{i}$, we now consider $n \mathrm{C}^{*}$-algebras $\mathcal{A}_{i}$. Their free product is defined exactly as in the case of groups, except that the morphism are now $*$-representations.

Definition 4.1.3. Let $\mathcal{A}_{i}$ be a collection of $n$ (unital) $\mathrm{C}^{*}$-algebras. Their free product ${ }^{*}{ }_{n} \mathcal{A}_{i}$ is the $\mathrm{C}^{*}$-algebra containing the $\mathcal{A}_{i}$ 's as subalgebras such that for every collection of $*$-homomorphisms $\phi_{i}: \mathcal{A}_{i} \rightarrow \mathcal{B}$ into any other $\mathrm{C}^{*}$-algebra $\mathcal{B}$, there exists a unique *-homomorphism $\phi:{ }_{n} \mathcal{A}_{i} \rightarrow \mathcal{B}$ extending the $\phi$ 's, that is $\left.\phi\right|_{\mathcal{A}_{i}}=\phi_{i}$.

The existence of the free product in the category of $\mathrm{C}^{*}$-algebras can be proven starting from the free products of algebras, which is defined along the lines of groups. The involution is defined canonically entry-wise, and the norm is taken as the supremum over all *-representations into the bounded operator on some Hilbert space. One readily sees that this defines a $\mathrm{C}^{*}$-algebra with the desired uniqueness property. In the case of universal group algebras, the existence can be also proven by using the free group product. That is, if $G_{i}$ is a collection of $n$ discrete groups, and $*_{n} G_{i}$ denotes their free product, then we consider the set of homomorphisms

$$
\phi_{i}: G_{i} \rightarrow \mathcal{U}(\mathcal{H})
$$

into the group of unitary operators on some Hilbert space $\mathcal{H}$. By the universality property of the free product, there exists a homomorphism

$$
\phi: *_{n} G_{i} \rightarrow \mathcal{U}(\mathcal{H})
$$

extending the $\phi_{i}$ 's. Let $\Gamma\left(*_{n} G_{i}\right)$ be the group ring of the free product, that is the $*$-algebra of elements of the form $\sum_{k} c_{k} w_{k}$ with $c_{k} \in \mathbb{C}, g_{\alpha_{i}} \in G_{i}$ and where $w_{k}$ is a general word 
in the free product, and $k$ ranges over a finite set. Then $\phi$ has an obvious extension to $\Gamma\left(*_{n} G_{i}\right)$, mapping its elements into bounded operators on $\mathcal{H}$. Norming the group ring by taken the supremum over all such maps gives rise to the universal group $\mathrm{C}^{*}$-algebra of the free product, $C^{*}\left(*_{n} G_{i}\right)$. Using the universality properties it is easy to see that we have an isomorphic identification

$$
C^{*}\left(*_{n} G_{i}\right) \simeq *_{n} C^{*}\left(G_{i}\right) .
$$

We apply this procedure to the $n$ copies of the group $\mathbb{Z}_{k}$, the group of integers with additional relation $a^{k}=e$. Using the discrete Fourier-transform, its universal group $\mathrm{C}^{*}$-algebra can be identified with the commutative $\mathrm{C}^{*}$-algebra $\ell_{k}^{\infty}$. Hence we obtain the identification

$$
C^{*}\left(*_{n} \mathbb{Z}_{k}\right) \simeq *_{n} C^{*}\left(\mathbb{Z}_{k}\right) \simeq *_{n} \ell_{k}^{\infty}
$$

the later being the free product of $n$ copies of the commutative and finite-dimensional $\mathrm{C}^{*}$-algebra $\ell_{k}^{\infty}$. We note that the free product obviously fails to be either commutative or finite-dimensional.

Embedding $\mathcal{L} \mathcal{M}_{n, k}$ into the free product. After the excursus introducing the notion of free products of groups and $\mathrm{C}^{*}$-algebras, we now prove the connection to the operator system of local measurements. We summarize the results in the following proposition.

Proposition 4.1.2. Let $\mathcal{L M}_{n, k}$ be the operator system of $n$ local measurements with $k$ outcomes each and let $*_{n} \ell_{k}^{\infty}$ be the free product of $n$ copies of the commutative $C^{*}$-algebra $\ell_{k}^{\infty}$. If $\Phi: \ell_{n}^{\infty} \otimes \ell_{k}^{\infty} \rightarrow \mathcal{B}(\mathcal{H})$ is the linear extension of the mapping

$$
\Phi: e_{i} \otimes e_{\alpha} \mapsto \pi_{i}\left(e_{\alpha}\right)
$$

where $\pi_{i}$ is the inclusion mapping of the $i$-th copy of $\ell_{k}^{\infty}$ into $*_{n} \ell_{k}^{\infty}$, then we have the following.

(i) The map $\Phi$ is completely positive and its kernel is equal to $\mathcal{Q}$.

(ii) The operator system $\mathcal{L M}_{n, k} \simeq \ell_{n}^{\infty} \otimes \ell_{k}^{\infty} / \operatorname{ker} \Phi$ is completely order isomorphic to the operator system generated by the elements $\pi_{i}\left(e_{\alpha}\right)$ inside the free product $*_{n} \ell_{k}^{\infty}$.

(iii) The unitary elements of the operator system $\mathcal{L} \mathcal{M}_{n, k}$ generate $*_{n} \ell_{k}^{\infty}$ as a $C^{*}$-algebra.

(iv) The operator system $\mathcal{L M}_{n, k}$ has the OSLLP.

Proof. ad (i).

The complete positivity follows from the fact that the map $\Phi$ is easily seen to be positive, and from the nuclearity of $\ell_{n}^{\infty} \otimes \ell_{k}^{\infty}$ in the category of $*$-AOU spaces. We obviously have that $\mathcal{Q} \subset \operatorname{ker} \Phi$, since for $e_{i}, e_{j} \in \ell_{n}^{\infty}$

$$
\Phi\left(\left(e_{i}-e_{j}\right) \otimes \sum_{\alpha} e_{\alpha}\right)=\Phi\left(e_{i} \otimes \sum_{\alpha} e_{\alpha}\right)-\Phi\left(e_{j} \otimes \sum_{\alpha} e_{\alpha}\right)=\pi_{i}(\mathbb{I})-\pi_{j}(\mathbb{I})=0,
$$

since the inclusions $\pi_{i}$ are unital. Conversely, assume that $a=\sum_{i, \alpha} a_{i, \alpha} e_{i} \otimes e_{\alpha} \in \operatorname{ker} \Phi$. Then we have

$$
0=\Phi(a)=\sum_{i, \alpha} a_{i, \alpha} \pi_{i}\left(e_{\alpha}\right)=\sum_{\alpha} \sum_{i} a_{i, \alpha} \pi_{i}\left(e_{\alpha}\right)
$$


and since the elements $\pi_{i}\left(e_{\alpha}\right)$ fulfill no further relation, the inner sum has to be independent of $\alpha, a_{i, \alpha}=a_{i}$. But this implies that $\sum_{i} a_{i}=0$, and hence $\operatorname{ker} \Phi \subset \mathcal{Q}$.

ad (ii).

Due to (i) and the first isomorphism theorem for completely positive maps, there exists a unique completely positive unital map $\tilde{\Phi}: \mathcal{L M}_{n, k} \rightarrow{ }_{n} \ell_{k}^{\infty}$ such that $\Phi=\tilde{\Phi} \circ q$, where $q: \ell_{n}^{\infty} \otimes \ell_{k}^{\infty} \rightarrow \ell_{n}^{\infty} \otimes \ell_{k}^{\infty} / \operatorname{ker} \Phi$ is the quotient map. We have to prove that $\tilde{\Phi}$ is a complete order embedding. Let $x \in M_{n} \otimes \mathcal{L} \mathcal{M}_{n, k}$ with $x \nsupseteq 0$, our goal is to prove that $\Phi(x) \nsupseteq 0$ as an element of $M_{n} \otimes *_{n} \ell_{k}^{\infty}$. By duality, this amounts to the fact that any completely positive map from $\mathcal{L M}_{n, k}$ to a finite-dimensional matrix algebra $M_{n}$ extends to a completely positive map from $*_{n} \ell_{k}^{\infty}$ into $M_{n}$. Note that we only have to consider finite-dimensional matrix algebras, as explained in the last chapter. So let $\phi: \mathcal{L} \mathcal{M}_{n, k} \rightarrow M_{n}$ be a completely positive map. By definition of the operator system $\mathcal{L M}_{n, k}$, this is nothing else but a collection of $n$ POVM's, that is, $n k$-tuples of operators $A_{i, \alpha} \in M_{n}$ such that

$$
A_{i, \alpha} \geq 0, \quad \sum_{\alpha} A_{i, \alpha}=\mathbb{I}_{n} \forall i \in\{1, \ldots, n\}
$$

It follows that for each $i$, the linear extension of the mapping

$$
\ell_{k}^{\infty} \ni e_{\alpha} \mapsto A_{i, \alpha}
$$

is completely positive and unital. Hence there exists a Hilbert space $\mathcal{K}$, a representation $\rho_{i}: \ell_{k}^{\infty} \rightarrow \mathcal{B}(\mathcal{K})$, and a isometry $v_{i}: \mathcal{H} \rightarrow \mathcal{K}$ such that

$$
A_{i, \alpha}=v_{i}^{*} \rho_{i}\left(e_{\alpha}\right) v_{i}
$$

which is of course nothing else but the Naimark dilation of the POVM $\left\{A_{i, \alpha}\right\}_{\alpha}$. It is easy to see that the Hilbert space $\mathcal{K}$ can be chosen as $\mathbb{C}^{n} \otimes \mathbb{C}^{k}$, independent of $i$. According to the discussion above, we have to find an isometry $V$ and a $*$-representation $\rho$ of the free product of the single copies of $\ell_{k}^{\infty}$ such that

$$
A_{i, \alpha}=v_{i}^{*} \rho_{i}\left(e_{\alpha}\right) v_{i}=V^{*} \rho\left(1 \cdot 1 \cdots e_{\alpha}^{i} \cdots 1\right) V
$$

where $1 \cdot 1 \cdots e_{\alpha}^{i} \cdots 1$ denotes the element of $*_{n} \ell_{k}^{\infty}$ which is the image of $e_{\alpha}$ under the inclusion $\pi_{i}$ of the $i$-th copy of $\ell_{k}^{\infty}$. Since for each $i, v_{i}$ is an isometry, its Halmos' dilation

$$
u_{i}=\left(\begin{array}{cc}
v_{i} & \left(1-v_{i} v_{i}^{*}\right)^{\frac{1}{2}} \\
0 & -v_{i}^{*}
\end{array}\right)
$$

is a unitary operator. We let $p$ be the projection onto the upper left corner of some element in $M_{2} \otimes M_{n}$, and define the $*$-homomorphism

$$
\tilde{\rho}_{i}: \ell_{k}^{\infty} \ni e_{\alpha} \mapsto u_{i}^{*} \rho_{i}\left(e_{\alpha}\right) \otimes \mathbb{I}_{2} u_{i} .
$$

Due to the universal property of the free product, there exists a $*$-representation $\rho: *_{n} \ell_{k}^{\infty} \rightarrow$ $M_{2} \otimes M_{n}$ such that $\rho$ reduces to $\tilde{\rho}_{i}$ if restricted to the $i$-th copy of $\ell_{k}^{\infty} \subset *_{n} \ell_{k}^{\infty}$. Hence we have that

$$
A_{i, \alpha}=p \rho\left(\pi_{i}\left(e_{\alpha}\right)\right) p^{*}
$$

and the assertion follows. 
ad (iii).

Due to (ii), the map $\Phi$ is a complete order embedding. Since the inclusions $\pi_{i}: \ell_{k}^{\infty} \rightarrow *_{n} \ell_{k}^{\infty}$ clearly map unitaries onto unitaries, the map $\Phi$ does the same. Now since each copy of $\ell_{k}^{\infty}$ is generated by a single unitary, the image of $\Phi$ generates each copy of $\ell_{k}^{\infty}$ in the free product and hence $*_{n} \ell_{k}^{\infty}$ itself.

ad (iv).

This follows from the work of Kavruk, [50], Theorem 6.8 together with the facts that $\mathcal{Q}$ contains 0 as the only possible positive element and that $\ell_{n}^{\infty} \otimes \ell_{k}^{\infty}$, being a commutative finite-dimensional $\mathrm{C}^{*}$-algebra, has the OSLLP. For the convenience of the reader, we also give a self-contained proof. So let $\mathcal{A}$ be a $\mathrm{C}^{*}$-algebra, $J \subset \mathcal{A}$ an ideal in $\mathcal{A}$, and $\phi$ : $\mathcal{L} \mathcal{M}_{n, k} \rightarrow \mathcal{A} / J$ be a completely positive map, given by the operators

$$
\mathcal{A} / J \ni A_{i, \alpha}=\phi\left(e_{i} \otimes e_{\alpha}+\mathcal{Q}\right) .
$$

Since an ideal in some $\mathrm{C}^{*}$-algebra is completely order proximinal in the operator sense, for each pair $(i, \alpha)$ there exists a "lift" of $A_{i, \alpha}$, a positive operator $B_{i, \alpha} \in \mathcal{A}$ such that $A_{i, \alpha}=q\left(B_{i, \alpha}\right)$, where $q: \mathcal{A} \rightarrow \mathcal{A} / J$ is the quotient map. It follows that

$$
\mathbb{I}=\sum_{\alpha} A_{i, \alpha}=q\left(\sum_{\alpha} B_{i, \alpha}\right),
$$

and for all $i$ there exists $h_{i} \in \mathcal{J}$ such that $\sum_{\alpha} B_{i, \alpha}=\mathbb{I}+h_{i}$. We now apply a trick introduced in [52], and let $k_{i} \in J$ such that $\mathbb{I}+h_{i}+k_{i}$ is positive and invertible. Then we define operators

$$
\tilde{B}_{i, \alpha}=\left(\mathbb{I}+h_{i}+k_{i}\right)^{-\frac{1}{2}}\left(B_{i, \alpha}+k_{i}\right)\left(\mathbb{I}+h_{i}+k_{i}\right)^{-\frac{1}{2}}
$$

which are positive for all $i, \alpha$ and furthermore satisfy $\sum_{\alpha} \tilde{B}_{i, \alpha}=\mathbb{I}$ for all $i$. Hence there is a completely positive map $\tilde{\phi}: \mathcal{L} \mathcal{M}_{n, k} \rightarrow \mathcal{A}$, such that $\tilde{\phi}\left(e_{i} \otimes e_{\alpha}+\mathcal{Q}\right)=\tilde{B}_{i, \alpha}$. Clearly, $\tilde{\phi}$ lifts $\phi$.

Tsirelson's problem in operator system language. Up to know, we only formalized the situation for one observer. Coming back to the Tsirelson setting, we now associate one copy of $\mathcal{L} \mathcal{M}_{n, k}$ to both Alice and Bob. That is, we consider two separated labs, in which Alice and Bob each make a choice of observables. Each party is again modeled by the operator system $\mathcal{L} \mathcal{M}_{n, k}$, more precisely by some completely positive unital map from $\mathcal{L M}_{n, k}$ into some Hilbert space $\mathcal{B}(\mathcal{H})$. They are operating on separate subsystems, which has the consequence that any choice Alice might make is compatible with any choice of Bob. Hence, if Alice chooses the observable $i$ with and observes outcome $\alpha$ and Bob chooses the observable $j$ and observes outcome $\beta$ their joint action corresponds to the elementary tensor

$$
\left(e_{i} \otimes e_{\alpha}+\mathcal{Q}\right) \otimes\left(e_{j} \otimes e_{\beta}+\mathcal{Q}\right) .
$$

For the motivation to model the situation by a tensor product, see the last chapter. The algebraic tensor product $\mathcal{L M}_{n, k} \otimes \mathcal{L} \mathcal{M}_{n, k}$ is not a priori an $*$-AOU space, even less an operator system, because we did not define what we mean by positive elements. Since we want to discuss possible quantum correlations between Alice and Bob, the stability under tensoring with a finite-dimensional quantum system is a valid assumption. This motivates the requirement that we are looking for possible operator system structures on the algebraic tensor product

$$
\mathcal{L} \mathcal{M}_{n, k} \otimes \mathcal{L} \mathcal{M}_{n, k}
$$


We introduced several choices for that in the foregoing chapter, the minimal, the commuting and the maximal tensor product. We recall that the minimal tensor product could be defined using the embedding

$$
\mathcal{L} \mathcal{M}_{n, k} \otimes_{\min } \mathcal{L M}_{n, k} \subset \mathcal{B}(\mathcal{H} \otimes \mathcal{H})
$$

if $\iota: \mathcal{L} \mathcal{M}_{n, k} \rightarrow \mathcal{B}(\mathcal{H})$ is a complete order embedding. Thus, any completely positive map $\phi: \mathcal{L M}_{n, k} \otimes_{\min } \mathcal{L} \mathcal{M}_{n, k} \rightarrow \mathcal{B}(\mathcal{K})$ into the bounded operators on some Hilbert space $\mathcal{K}$ can always be extended to $\mathcal{B}(\mathcal{H} \otimes \mathcal{H})$, since $\mathcal{B}(\mathcal{K})$ is injective. Using Stinespring's dilation, we obtain an isometry $V: \mathcal{K} \rightarrow \mathcal{H} \otimes \mathcal{H}$ such that

$$
\phi(a \otimes b)=V^{*} \iota(a) \otimes \iota(b) V .
$$

If now $\omega$ is some state of the quantum system modeled by the Hilbert space $\mathcal{K}^{3}$, then setting $\tilde{\omega}=\omega\left(V^{*} . V\right)$ gives rise to state on $\mathcal{B}(\mathcal{H} \otimes \mathcal{H})$. Hence any quantum representation of $\mathcal{L} \mathcal{M}_{n, k} \otimes_{\text {min }} \mathcal{L M}_{n, k}$ can be brought into tensor product form.

Now consider the commuting tensor product, $\mathcal{L M}_{n, k} \otimes_{c} \mathcal{L M}_{n, k}$. By definition, any completely positive map $\phi: \mathcal{L M}_{n, k} \otimes_{c} \mathcal{L} \mathcal{M}_{n, k} \rightarrow \mathcal{B}(\mathcal{K})$ for some Hilbert space $\mathcal{K}$, is of the form

$$
\phi(a \otimes b)=\phi_{A}(a) \cdot \phi_{B}(b)
$$

where $\phi_{A, B}$ are completely positive maps from a single copy of $\mathcal{L} \mathcal{M}_{n, k}$ into $\mathcal{B}(\mathcal{K})$ such that their ranges commute. It follows that any quantum representation of $\mathcal{L M}_{n, k} \otimes_{c} \mathcal{L M}_{n, k}$ is of commuting form.

Hence we conclude that if both operator system structures, the minimal and the commuting, coincide,

$$
\mathcal{L} \mathcal{M}_{n, k} \otimes_{\min } \mathcal{L} \mathcal{M}_{n, k} \simeq \mathcal{L} \mathcal{M}_{n, k} \otimes_{c} \mathcal{L} \mathcal{M}_{n, k}
$$

then Tsirelson's problem has a positive answer. For the converse, note that a quantum representation of either side only concerns elements of the first matrix level, but the upper equality is understood as in the operator system sense, that is we have

$$
M_{n} \otimes \mathcal{L} \mathcal{M}_{n, k} \otimes_{\min } \mathcal{L M}_{n, k} \simeq M_{n} \otimes \mathcal{L} \mathcal{M}_{n, k} \otimes_{c} \mathcal{L} \mathcal{M}_{n, k}
$$

for all $n \in \mathbb{N}$. This asks for the stability of Tsirelson's problem under tensoring with a third, albeit finite-dimensional, matrix system. See [30] for possible physical meanings in the operational sense. Thus, Tsirelson's problem is more a question on tensor product cones of $*$-AOU spaces, rather than about operator systems. It is however unclear whether there is really a difference in both setups, that is, we do not know whether Tsirelson's problem can be true on the first matrix level, but simultaneously being false on higher matrix levels.

\subsection{Connecting Tsirelson's problem with Kirchberg's conjecture}

In this section, we will combine the insights from the previous sections and show that, in some sense, both problems, Tsirelson's and Connes', are indeed equivalent. More specifically, we will prove the following theorem:

\footnotetext{
${ }^{3}$ Which we might have obtained by the GNS construction applied to the system $\mathrm{C}^{*}$-algebra with respect to the physical state $\omega$.
} 
Theorem 4.2.1. Let $C^{*}\left(\mathbb{F}_{n}\right)$ be the universal $C^{*}$-algebra of the free group of order $n$, and let $\mathcal{L M}_{n, k}$ be the operator system of $n$ local measurements with $k$ outcomes each, for $n, k \in \mathbb{N}$. Then the following are equivalent.

(i) For all natural numbers $n \in \mathbb{N}$ we have the following $*$-isomorphism,

$$
C^{*}\left(\mathbb{F}_{n}\right) \otimes_{\min } C^{*}\left(\mathbb{F}_{n}\right)=C^{*}\left(\mathbb{F}_{n}\right) \otimes_{\max } C^{*}\left(\mathbb{F}_{n}\right) .
$$

(ii) For all pairs of natural numbers $n, k \in \mathbb{N}$ we have the following complete order isomorphism,

$$
\mathcal{L M}_{n, k} \otimes_{\min } \mathcal{L} \mathcal{M}_{n, k} \simeq \mathcal{L} \mathcal{M}_{n, k} \otimes_{c} \mathcal{L} \mathcal{M}_{n, k}
$$

Thus, according to the discussion in the last section, Tsirelson's problem has a positive answer if the Connes' embedding problem holds, or equivalently, if Kirchberg's QWEP conjecture is true. As noted above, the converse is not true if we only consider the first matrix level. In order to prove the equivalence stated in Theorem 4.2.1, we start by reformulating equation 4.3 in terms of the free product $*_{n} \ell_{k}^{\infty}$, since the operator system $\mathcal{L M}_{n, k}$ embeds completely isomorphic into the free product. The result is certainly known to experts, but we prove it for the sake of completeness.

Lemma 4.2.2. Let $n, k \in \mathbb{N}, C^{*}\left(\mathbb{F}_{n}\right)$ be the universal $C^{*}$-algebra of the free group of order $n$, and let $*_{n} \ell_{k}^{\infty}$ be the free product of $n$ copies of the commutative $C^{*}$-algebra $\ell_{k}^{\infty}$. Then the following are equivalent.

(i) The minimal and the maximal tensor product of $C^{*}\left(\mathbb{F}_{n}\right)$ with itself are $*$-isomorphic,

$$
C^{*}\left(\mathbb{F}_{n}\right) \otimes_{\min } C^{*}\left(\mathbb{F}_{n}\right)=C^{*}\left(\mathbb{F}_{n}\right) \otimes_{\max } C^{*}\left(\mathbb{F}_{n}\right) .
$$

(ii) The same holds for the tensor product of $*_{n} \ell_{k}^{\infty}$ with itself,

$$
*_{n} \ell_{k}^{\infty} \otimes_{\min } *_{n} \ell_{k}^{\infty}=*_{n} \ell_{k}^{\infty} \otimes_{\max } *_{n} \ell_{k}^{\infty},
$$

for all natural numbers $k \in \mathbb{N}$.

Proof. ad (i) $\Rightarrow$ (ii).

We note that the commutative $\mathrm{C}^{*}$-algebra $\ell_{k}^{\infty}$, considered as an operator system, has the OSLLP, which for $\mathrm{C}^{*}$-algebras is equivalent to the LLP. By [73], taking free products preserves the LLP, which implies that $*_{n} \ell_{k}^{\infty}$ has the LLP. It follows from Proposition 3.19 (vi) in [69] that, assuming that Kirchberg's conjecture holds, a $\mathrm{C}^{*}$-algebra with the LLP has also the WEP, which implies that as an operator system it has the DCEP. Hence (i) follows from Lemma 3.2.8.

ad (ii) $\Rightarrow$ (i).

We first observe that $\mathbb{F}_{n} \simeq *_{n} \mathbb{Z}$, which follows easily from comparing the relation of generators. By Fourier-transform, the group $\mathrm{C}^{*}$-algebra of $\mathbb{Z}$ is equal to the complex-valued continuous functions on the unit circle, $C^{*}(\mathbb{Z})=C(\mathbb{T})$. According to the discussion in the last section on group $\mathrm{C}^{*}$-algebras of free products, we have then

$$
C^{*}\left(\mathbb{F}_{N}\right)=C^{*}\left(*_{n} \mathbb{Z}\right)=*_{n} C^{*}(\mathbb{Z})=*_{n} C(\mathbb{T}) .
$$

Since $C(\mathbb{T})$ is a commutative $\mathrm{C}^{*}$-algebra, it is a nuclear $*$-AOU space, and as discussed in the chapter of $*$-AOU spaces, this implies that it admits a net $\Psi_{\lambda}$ of completely positive 
unital maps converging point-wise to the identity, each of them admitting a factorization $\Psi_{\lambda}=v_{\lambda} u_{\lambda}$. Here $u_{\lambda}: C(\mathbb{T}) \rightarrow \ell_{\infty}^{m_{\lambda}}$ and $v_{\lambda}: \ell_{\infty}^{m_{\lambda}} \rightarrow C(\mathbb{T})$ are both completely positive and unital maps. Now according to a result by Boca, a collection of unital completely positive maps on each copy extends to the free product, see $[11,10]$. Therefore we deduce from our assumption that for every $z \in C^{*}\left(\mathbb{F}_{n}\right) \otimes C^{*}\left(\mathbb{F}_{n}\right)$ and $\lambda$ we have

$$
\begin{aligned}
& \left\|\left(*_{N} \Psi_{\lambda} \otimes *_{N} \Psi_{\lambda}\right)(z)\right\|_{C^{*}\left(\mathbb{F}_{n}\right) \otimes_{\max } C^{*}\left(\mathbb{F}_{n}\right)} \\
& \leq\left\|\left(\left(*_{n} u_{\lambda}\right) \otimes\left(*_{n} u_{\lambda}\right)\right)(z)\right\|_{*_{n} \ell_{k}^{\infty} \otimes_{\max } *_{n} \ell_{k}^{\infty}} \\
& =\left\|\left(\left(*_{n} u_{\lambda}\right) \otimes\left(*_{n} u_{\lambda}\right)\right)(z)\right\|_{*_{n}} \ell_{k}^{\infty} \otimes_{\min *_{n}} \ell_{k}^{\infty} \\
& \leq\|z\|_{C^{*}\left(\mathbb{F}_{n}\right) \otimes_{\min } C^{*}\left(\mathbb{F}_{n}\right) .}
\end{aligned}
$$

It is easy to see that for tensors $z=\sum_{k} a_{k} \otimes b_{k}$ we have norm convergence along the net. By density we obtain the assertion.

Due to Proposition 4.1.2, we already know that the operator system $\mathcal{L} \mathcal{M}_{n, k}$ embeds completely isomorphically into the free product $*_{n} \ell_{k}^{\infty}$. It follows then from the injectivity of the minimal tensor product, see the last chapter, that the canonical embedding $\mathcal{L M}_{n, k} \otimes$ $\mathcal{L} \mathcal{M}_{n, k} \rightarrow *_{n} \ell_{k}^{\infty} \otimes_{\min } *_{n} \ell_{k}^{\infty}$ extends to a complete order embedding,

$$
\mathcal{L} \mathcal{M}_{n, k} \otimes_{\min } \mathcal{L} \mathcal{M}_{n, k} \subset *_{n} \ell_{k}^{\infty} \otimes_{\min } *_{n} \ell_{k}^{\infty} .
$$

Assuming the same for the commutative tensor product between $\mathcal{L} \mathcal{M}_{n, k}$ with itself, with the minimal $\mathrm{C}^{*}$-tensor product on the right hand side replaced by the maximal $\mathrm{C}^{*}$-tensor product, and the validity of the QWEP conjecture, we obtain the chain of inclusions

$$
\mathcal{L} \mathcal{M}_{n, k} \otimes_{\min } \mathcal{L} \mathcal{M}_{n, k} \subset *_{n} \ell_{k}^{\infty} \otimes_{\min } *_{n} \ell_{k}^{\infty}=*_{n} \ell_{k}^{\infty} \otimes_{\max } *_{n} \ell_{k}^{\infty} \supset \mathcal{L} \mathcal{M}_{n, k} \otimes_{c} \mathcal{L} \mathcal{M}_{n, k},
$$

which implies that

$$
\mathcal{L} \mathcal{M}_{n, k} \otimes_{\text {min }} \mathcal{L M}_{n, k} \simeq \mathcal{L} \mathcal{M}_{n, k} \otimes_{c} \mathcal{L} \mathcal{M}_{n, k}
$$

Hence, Proposition 4.2.3 below asserts that Tsirelson's problem has a positive solution if all $\mathrm{C}^{*}$-algebras have QWEP.

Proposition 4.2.3. Let $\mathcal{L M}_{n, k}$ be the operator system of $n$ local measurements with $k$ outcomes each, for $n, k \in \mathbb{N}$ and let $*_{n} \ell_{k}^{\infty}$ be the free product of $n$ copies of the commutative $C^{*}$-algebra $\ell_{k}^{\infty}$. Then the canonical inclusion $\mathcal{L M}_{n, k} \otimes \mathcal{L} \mathcal{M}_{n, k} \rightarrow *_{n} \ell_{k}^{\infty} \otimes_{\max } *_{n} \ell_{k}^{\infty}$ extends to a complete order embedding,

$$
\mathcal{L} \mathcal{M}_{n, k} \otimes_{c} \mathcal{L} \mathcal{M}_{n, k} \subset *_{n} \ell_{k}^{\infty} \otimes_{\max } *_{n} \ell_{k}^{\infty}
$$

Proof. The proof idea is again based on Halmos' dilation, combined with Arveson's commutant lifting theorem. We let $*_{n} \ell_{k}^{\infty} \otimes_{\max } *_{n} \ell_{k}^{\infty}$ be faithfully represented on some Hilbert space $\mathcal{H}$. By the definition of the maximal tensor product, the representation is given by a pair of commuting $*$-representations $\pi_{1,2}: *_{n} \ell_{k}^{\infty} \rightarrow \mathcal{B}(\mathcal{H})$. If $\iota: \mathcal{L} \mathcal{M}_{n, k} \rightarrow *_{n} \ell_{k}^{\infty}$ denotes the inclusion, then it follows that $\left(\pi_{1} \circ \iota, \pi_{2} \circ \iota\right)$ is a pair of completely positive unital maps with commuting ranges, and

$$
\mathcal{L M}_{n, k} \otimes_{c} \mathcal{L} \mathcal{M}_{n, k} \ni a \otimes b \mapsto \pi_{1} \circ \iota(a) \cdot \pi_{2} \circ \iota(b)
$$


is completely positive and unital. As in the proof of Proposition 4.1.2, we have to show that each such map can be extended to a completely positive map of $*_{n} \ell_{k}^{\infty} \otimes_{\max } *_{n} \ell_{k}^{\infty}$ into $\mathcal{B}(\mathcal{H})$. Let $A_{i, \alpha}, B_{j, \beta}$ be the images of the elementary generators of $\mathcal{L} \mathcal{M}_{n, k}$, i.e.

$$
A_{i, \alpha}=\pi_{1} \circ \iota\left(e_{i} \otimes e_{\alpha}+\mathcal{Q}\right), \quad B_{j, \beta}=\pi_{1} \circ \iota\left(e_{j} \otimes e_{\beta}+\mathcal{Q}\right) .
$$

We then apply the trick from the proof of Proposition 4.1.2, ad (ii), first to the operators $A_{i, \alpha}$. That is, since for each $i$, they are the images of completely positive maps from $\ell_{k}^{\infty}$ into $\mathcal{B}(\mathcal{H})$, there exists a Hilbert space $\mathcal{K}$, an isometry $v_{i}: \mathcal{H} \rightarrow \mathcal{K}$, a $*$-representation $\rho_{i}: \ell_{k}^{\infty} \rightarrow \mathcal{B}(\mathcal{K})$ such that

$$
A_{i, \alpha}=v_{i}^{*} \rho_{i}\left(e_{\alpha}\right) v_{i} .
$$

Now, since for each $i, v_{i}$ is an isometry, we again apply Halmos' dilation

$$
u_{i}=\left(\begin{array}{cc}
v_{i} & \left(1-v_{i} v_{i}^{*}\right)^{\frac{1}{2}} \\
0 & -v_{i}^{*}
\end{array}\right)
$$

resulting in a unitary operator. We let $p_{1}$ be the projection onto the upper left corner of some element in $M_{2} \otimes \mathcal{B}(\mathcal{H})=\mathcal{B}\left(\mathbb{C}^{2} \otimes \mathcal{H}\right)$, and define the $*$-homomorphism

$$
\tilde{\rho}_{i}: \ell_{k}^{\infty} \ni e_{\alpha} \mapsto u_{i}^{*} \rho_{i}\left(e_{\alpha}\right) \otimes \mathbb{I}_{2} u_{i} .
$$

It now follows again due to the universal property of the free product that there is a $*$ representation $\rho: *_{n} \ell_{k}^{\infty} \rightarrow \mathcal{B}\left(\mathbb{C}^{2} \otimes \mathcal{H}\right)$ such that $\rho$ reduces to $\tilde{\rho}_{i}$ if restricted to the $i$-th copy of $\ell_{k}^{\infty} \subset *_{n} \ell_{k}^{\infty}$, resulting in

$$
A_{i, \alpha}=p_{1} \rho\left(\pi_{i}\left(e_{\alpha}\right)\right) p_{1}^{*} .
$$

We note that the operators on Bob's side, $B_{j, \beta}$, lie in the commutant of the completely positive unital map $p_{1} \rho(.) p_{1}^{*}$. By Arveson's commutant lifting theorem, there is a corresponding $*$-representation $\delta$ of this commutant to $\mathcal{B}\left(\mathbb{C}^{2} \otimes \mathcal{H}\right)$ such that

$$
\delta:\left(p_{1} \rho\left(*_{n} \ell_{k}^{\infty}\right) p_{1}^{*}\right)^{\prime} \rightarrow\left(\rho\left(*_{n} \ell_{k}^{\infty}\right)\right)^{\prime} .
$$

Hence, if we let $\tilde{B}_{j, \beta}$ be the images of the generators $e_{j} \otimes e_{\beta}+\mathcal{Q}$ under the composition $\delta \circ \pi_{2} \circ \iota$, we obtain a quantum representation of $\mathcal{L} \mathcal{M}_{n, k}$ on the commutant of $\rho\left(*_{n} \ell_{k}^{\infty}\right)$. We then apply the same procedure as above, i.e. using first Naimark's dilation of the POVM's $\tilde{B}_{j, \beta} \in \mathcal{B}\left(\mathbb{C}^{2} \otimes \mathcal{H}\right)$ and then constructing Halmos' dilation of the corresponding isometries. Note that if we choose a "second" copy of the dilating space $\mathbb{C}^{2}$, then we obtain, again from the universal property of the free product, a *-representation $\sigma: *_{n} \ell_{k}^{\infty} \rightarrow \mathcal{B}\left(\mathbb{C}^{4} \otimes \mathcal{H}\right)$, commuting with $\rho \otimes \mathbb{I}_{2}: *_{n} \ell_{k}^{\infty} \rightarrow \mathcal{B}\left(\mathbb{C}^{4} \otimes \mathcal{H}\right)$. We then let $p_{2}$ be the projection onto the first coordinate of the second copy of the dilating space $\mathbb{C}^{2}$, obtaining

$$
A_{i, \alpha} \cdot B_{j, \beta}=p_{1} \otimes p_{2}\left[\rho\left(\pi_{i}\left(e_{\alpha}\right)\right) \cdot \sigma\left(\pi_{i}\left(e_{\alpha}\right)\right)\right] p_{1}^{*} \otimes p_{2}^{*}
$$

where as before, $\pi_{i}$ is the inclusion mapping of the $i$-th copy of $\ell_{k}^{\infty}$ into $*_{n} \ell_{k}^{\infty}$. The right hand side is easily seen to be the restriction of some completely positive map of $*_{n} \ell_{k}^{\infty} \otimes_{\max } *_{n} \ell_{k}^{\infty}$ into $\mathcal{B}(\mathcal{H})$ on the subspace generated by the elements $\iota\left(e_{i} \otimes e_{\alpha}+\mathcal{Q}\right) \otimes \iota\left(e_{j} \otimes e_{\beta}+\mathcal{Q}\right)$, which proves the assertion. 
We finish the proof of Theorem 4.2.1 by showing that the isomorphism between the minimal and the commuting tensor product structure on two copies of the operator system $\mathcal{L} \mathcal{M}_{n, k}$ implies (ii), and hence (i) in the just mentioned theorem. The proof is identical to a nice and short argument of Kavruk et.al. [52], where it was first applied for operator systems. The essential argument is due to Choi's theory of multiplicative domains, and was applied in a similar way in the context of operator spaces by Pisier [73].

Lemma 4.2.4. Let $\mathcal{L M}_{n, k}$ be the operator system of $n$ local measurements with $k$ outcomes each, for $n, k \in \mathbb{N}$ and let $*_{n} \ell_{k}^{\infty}$ be the free product of $n$ copies of the commutative $C^{*}$-algebra $\ell_{k}^{\infty}$. Then the complete order isomorphism

$$
\mathcal{L M}_{n, k} \otimes_{\min } \mathcal{L} \mathcal{M}_{n, k} \simeq \mathcal{L} \mathcal{M}_{n, k} \otimes_{c} \mathcal{L} \mathcal{M}_{n, k}
$$

implies the $C^{*}$-isomorphism

$$
{ }_{n} \ell_{k}^{\infty} \otimes_{\min } *_{n} \ell_{k}^{\infty}=*_{n} \ell_{k}^{\infty} \otimes_{\max } *_{n} \ell_{k}^{\infty}
$$

Proof. We know from Proposition 4.1.2 (iii) that the unitary elements of $\mathcal{L M}_{n, k}$ generated the free product $*_{n} \ell_{k}^{\infty}$. Is is easy to see that this also holds for the tensor product, i.e. we have that the unitary elements of $\mathcal{L} \mathcal{M}_{n, k} \otimes_{\min } \mathcal{L} \mathcal{M}_{n, k}$ generate $*_{n} \ell_{k}^{\infty} \otimes_{\min } *_{n} \ell_{k}^{\infty}$, and the unitary elements of $\mathcal{L} \mathcal{M}_{n, k} \otimes_{c} \mathcal{L} \mathcal{M}_{n, k}$ generate $*_{n} \ell_{k}^{\infty} \otimes_{\max } *_{n} \ell_{k}^{\infty}$, respectively. Since moreover, the inclusion $\iota: \mathcal{L} \mathcal{M}_{n, k} \rightarrow{ }_{n} \ell_{k}^{\infty}$ maps unitaries onto unitaries, the tensor product maps $\iota \otimes_{\min } \iota$, resp. $\iota \otimes_{c} \iota$ do the same, and we have for each pair of unitaries $V, U \in \mathcal{L} \mathcal{M}_{n, k}$

$$
\mathbb{I}=\iota \otimes_{\min } \iota\left(U^{*} U \otimes V^{*} V\right)=\iota \otimes_{c} \iota\left(U^{*} \otimes V^{*}\right) \cdot \iota \otimes_{c} \iota(U \otimes V)
$$

which implies that the unitary generators are in the multiplicative domain of the complete order isomorphism between $\mathcal{L} \mathcal{M}_{n, k} \otimes_{\min } \mathcal{L} \mathcal{M}_{n, k}$ and $\mathcal{L} \mathcal{M}_{n, k} \otimes_{c} \mathcal{L} \mathcal{M}_{n, k}$. By Choi's theory of multiplicative domains, see e.g. the book of Paulsen, [70], the identification map extends to a $\mathrm{C}^{*}$-isomorphism between the generated algebras.

Approximation by finite-dimensional representations Since Kirchberg's QWEP conjecture is still open, it is naturally to ask for $\mathrm{C}^{*}$-algebras $\mathcal{A}$ such that Tsirelson's problem, applied to these specific examples, has a positive answer. That is, assume that the physical system on Alice' side is modeled by a $\mathrm{C}^{*}$-algebra $\mathcal{A}$, and the system is in the state $\omega$. After applying the GNS construction, we obtain a Hilbert space $\mathcal{H}$, and a representation $\pi$ of $\mathcal{A}$ on $\mathcal{H}$. If we denote by $\pi(\mathcal{A})^{\prime}$ the commutant of $\pi(\mathcal{A}) \subset \mathcal{B}(\mathcal{H})$, then Bob's algebra is contained in $\pi(\mathcal{A})^{\prime}$. As discussed above, the situation that both of them perform a finite number of measurements with a finite number of outcomes each, is modeled by a pair of completely positive and unital maps $\left(\phi, \phi^{\prime}\right)$ from $\mathcal{L} \mathcal{M}_{n, k}$ (for suitably chosen $n, k$ ) into the respective algebras. Hence we may ask the question, if $\phi: \mathcal{L} \mathcal{M}_{n, k} \rightarrow \pi(\mathcal{A})$ and $\phi^{\prime}: \mathcal{L} \mathcal{M}_{n, k} \rightarrow \pi(\mathcal{A})^{\prime}$ are completely positive and unital maps giving rise to correlations tables of commuting form, under which conditions on $\mathcal{A}$ does there also exists a quantum representation of tensor product form?

Under the assumption that the underlying Hilbert space $\mathcal{H}$ is finite-dimensional it was shown by Tsirelson that there is no difference between the two models. Without modifications, the proof carries over to the slightly more general case that the images of $\phi$ resp. $\phi^{\prime}$ in $\mathcal{B}(\mathcal{H})$ generate a finite-dimensional von-Neumann algebra. That is, the set of 
all linear combinations and products of elements forms a finite-dimensional vector space. Obviously, this requirement is in particular fulfilled if the underlying Hilbert space $\mathcal{H}$ is of finite dimension.

Proposition 4.2.5. Let $\left\{A_{i, \alpha}\right\} \subset \mathcal{B}(\mathcal{H})$, and $\left\{B_{j, \beta}\right\} \subset \mathcal{B}(\mathcal{H})$ be finite, commuting sets of positive operators, each generating a finite-dimensional von-Neumann algebra.

Then there exists a finite-dimensional Hilbert space $\overline{\mathcal{H}}$, such that $\mathcal{H} \subset \overline{\mathcal{H}}$, which can be decomposed as $\overline{\mathcal{H}}=\mathcal{H}_{A} \otimes \mathcal{H}_{B}$ such that $\left\{A_{i, \alpha}\right\}$ can be mapped isomorphically into $\mathcal{B}\left(\mathcal{H}_{A}\right)$ and correspondingly $\{B\}_{j, \beta}$ into $\mathcal{B}\left(\mathcal{H}_{B}\right)$.

Proof. The proof technique, which we call "doubling the center", was already described by Tsirelson [87], but we include it here for the convenience of the reader. Let $\mathcal{A}_{X}$ resp. $\mathcal{A}_{Y}$ be the algebra generated by the sets $\left\{A_{i, \alpha}\right\}$ resp. $\left\{B_{j, \beta}\right\}$. Obviously, $\mathcal{A}_{Y}$ lies in the commutant of $\mathcal{A}_{X}, \mathcal{A}_{X}^{\prime}$. Since $\mathcal{A}_{X}$ as well as $\mathcal{A}_{X}^{\prime}$ are finite-dimensional von-Neumann algebras, they can be decomposed into a direct sum of type I factors, $\mathcal{A}_{X}=\oplus_{k} \mathcal{A}_{k}$ resp. $\mathcal{A}_{X}^{\prime}=\oplus_{k} \mathcal{A}_{k}^{\prime}$. Correspondingly, the Hilbert space $\mathcal{H}$ can be decomposed into a direct sum $\mathcal{H}=\oplus_{k} \mathcal{H}_{k}$. Now, using the fact that $\mathcal{A}_{k}$ is of type I for each $k$, we can further decompose each Hilbert space $\mathcal{H}_{k}$ into a tensor product, $\mathcal{H}_{k}=\mathcal{H}_{k}^{1} \otimes \mathcal{H}_{k}^{2}$ such that $\mathcal{A}_{k}$ (resp. $\mathcal{A}_{k}^{\prime}$ ) acts non-trivially only on $\mathcal{H}_{k}^{1}$ (resp. $\mathcal{H}_{k}^{2}$ ). An elementary proof of this, as well as some physical examples, can also be found in [91]. What remains is to embed the Hilbert space $\mathcal{H}$ into $\left(\oplus_{k} \mathcal{H}_{k}^{1}\right) \otimes\left(\oplus_{l} \mathcal{H}_{l}^{2}\right)$. Thus, $\mathcal{A}_{X}$ (resp. $\left.\mathcal{A}_{X}^{\prime}\right)$ can be mapped isomorphically into $\mathcal{B}\left(\oplus_{k} \mathcal{H}_{k}^{1}\right)\left(\right.$ resp. $\mathcal{B}\left(\oplus_{k} \mathcal{H}_{k}^{2}\right)$ ).

Hence, in finite dimension, every quantum correlation function derived from commuting observables can also be represented by observables having tensor product form. In abstract words, this is a consequence of the fact that all von-Neumann algebras represented on a finite-dimensional Hilbert space are of type I. Indeed, as mentioned in the proof, the existence of a tensor decomposition is characteristic for type I von-Neumann algebras. Thus, one might be tempted to conclude that the problem is connected to the possible types of von-Neumann algebras occurring in the description of physical systems. However, the next proposition asserts that the class of algebras for which both models agree is much larger.

Proposition 4.2.6. Let $\mathcal{L M}_{n, k}$ be the operator system of $n$ local measurements with $k$ outcomes each, for $n, k \in \mathbb{N}$ and let $\mathcal{N} \subset \mathcal{B}(\mathcal{H})$ be a von Neumann algebra which is $Q W E P$. Furthermore, let $\phi: \mathcal{L M}_{n, k} \rightarrow \mathcal{N}$ and $\phi^{\prime}: \mathcal{L M}_{n, k} \rightarrow \mathcal{N}^{\prime}$ be a pair of completely positive and unital maps. Then the mapping

$$
\mathcal{L M}_{n, k} \otimes \mathcal{L} \mathcal{M}_{n, k} \ni \sum_{i} a_{i} \otimes b_{i} \mapsto \sum_{i} \phi\left(a_{i}\right) \phi^{\prime}\left(b_{i}\right)
$$

extends to a completely positive and unital mapping from $\mathcal{L} \mathcal{M}_{n, k} \otimes_{\text {min }} \mathcal{L} \mathcal{M}_{n, k}$ into $\mathcal{B}(\mathcal{H})$.

Proof. Let $\mathcal{B}$ be a $\mathrm{C}^{*}$-algebra with the WEP, $J \subset \mathcal{B}$ an ideal in $\mathcal{B}$ such that $\mathcal{N} \simeq \mathcal{B} / J$. Since by Proposition 4.1.2 (iv), the operator system $\mathcal{L M}_{n, k}$ has the OSLLP, there is a completely positive and unital lifting $\tilde{\phi}: \mathcal{L} \mathcal{M}_{n, k} \rightarrow \mathcal{B}$ of the map $\phi: \mathcal{L} \mathcal{M}_{n, k} \rightarrow \mathcal{N}$. If we let $q: \mathcal{B} \rightarrow \mathcal{N}$ be the quotient map, then we have a completely positive and unital map

$$
q \otimes \phi^{\prime}: \mathcal{B} \otimes_{c} \mathcal{L M}_{n, k} \rightarrow \mathcal{B}(\mathcal{H}) .
$$

Since any $\mathrm{C}^{*}$-algebra having the WEP also has, if considered as an operator system, the DCEP, we can apply Lemma 3.2.8 and the map extends to a completely positive and unital 
map $\psi: \mathcal{B} \otimes_{\text {min }} \mathcal{L M}_{n, k} \rightarrow \mathcal{B}(\mathcal{H})$. Hence the composition $\psi \circ\left(\tilde{\phi} \otimes \operatorname{id}_{\mathcal{L} \mathcal{M}_{n, k}}\right)$ is the desired mapping.

Coming back to the physical example discussed before, assume that the von Neumann algebra $\pi(\mathcal{A})^{\prime \prime}$ generated by $\pi(\mathcal{A})$ is QWEP. Slightly changing the notation, we let $\omega$ be the state on $\mathcal{B}(\mathcal{H})$, which if restricted to $\pi(\mathcal{A})^{\prime \prime}$ and its commutant $\pi(\mathcal{A})^{\prime}$, gives rise to some correlation table of commuting form. Then we may apply the foregoing proposition to $\pi(\mathcal{A})^{\prime \prime}$ and obtain a completely positive unital extension $\psi: \mathcal{L} \mathcal{M}_{n, k} \otimes_{\min } \mathcal{L M}_{n, k} \rightarrow \mathcal{B}(\mathcal{H})$ of the pair $\left(\phi, \phi^{\prime}\right)$,

$$
\left.\psi\right|_{\mathcal{L M}_{n, k} \otimes \mathbb{I}}=\left.\phi \quad \psi\right|_{\mathbb{I} \otimes \mathcal{L} \mathcal{M}_{n, k}}=\phi^{\prime} .
$$

Let $\mathcal{K}$ be a Hilbert space such that $\mathcal{L} \mathcal{M}_{n, k} \subset \mathcal{B}(\mathcal{K})$, then we have a complete order embed$\operatorname{ding} \mathcal{L M}_{n, k} \otimes_{\min } \mathcal{L} \mathcal{M}_{n, k} \subset \mathcal{B}(\mathcal{K} \otimes \mathcal{K})$, such that the map $\psi$ is extendable to $\mathcal{B}(\mathcal{K} \otimes \mathcal{K})$. It follows that $\omega \circ \psi$ is a state on $\mathcal{B}(\mathcal{K} \otimes \mathcal{K})$, giving rise to the same correlation table as the state $\omega$ on $\mathcal{B}(\mathcal{H})$. Hence Proposition 4.2.6 provides us with a second proof that Kirchberg's QWEP conjecture implies a positive solution to Tsirelson's problem, since according to the conjecture, all $\mathrm{C}^{*}$-algebras are quotients of algebras having the weak expectation property.

But even if Kirchberg's conjecture fails to be true, there are many $\mathrm{C}^{*}$-algebras occurring in physics which are know to be QWEP. This class includes any fermionic system, quantum spin systems, the CHSH case and usual models from quantum field theory. In particular, nuclear $\mathrm{C}^{*}$-algebras and hyperfinite von Neumann algebras are QWEP, and even have the WEP.

We recall that a $\mathrm{C}^{*}$-algebra $\mathcal{A}$ is called nuclear, if there is a unique tensor product norm on $\mathcal{A} \otimes \mathcal{B}$ for any other $\mathrm{C}^{*}$-algebra $\mathcal{B}$. The class of nuclear $\mathrm{C}^{*}$-algebras contains many physical examples, and the following listing just mentions a few of them.

(i) All $\mathrm{C}^{*}$-algebras describing fermionic systems, i.e. $\mathrm{C}^{*}$-algebras generated by elements fulfilling the canonical anti-commutation relations.

(ii) The $\mathrm{C}^{*}$-algebra generated by two projections, corresponding to the case that Alice has two measurement devices with two outcomes each [76].

(iii) Uniformly hyperfinite algebras describing infinite spin systems [53].

For more information about nuclear $\mathrm{C}^{*}$-algebras we recommend the survey by Rordam, [78]. We now consider the case of hyperfinite von Neumann algebras. Recall that a von Neumann algebra $\mathcal{N}$ is called hyperfinite, if there exists an increasing net of finite dimensional matrix algebras such that the union is weak-*-dense in $\mathcal{N}$. There exists many examples of hyperfinite algebras in physics, we name a few.

(i) The unique hyperfinite type $I I_{1}$ factor describing the local algebras of an infinite chain of maximally entangled two-qubit states [54].

(ii) The factors occurring in the construction of algebraic quantum field theory are usually isomorphic to the unique type $I I I_{1}$ factor [13].

Since there exist hyperfinite von-Neumann algebras of any type [3], this shows in particular that the problem is not connected to types of von-Neumann algebras. Rather, Tsirelson's problem has a positive answer for all von Neumann algebras being the quotient 
of a $\mathrm{C}^{*}$-algebra with the weak expectation property. Since we know by the work of Kirchberg that a finite von Neumann algebra can be embedded into an ultraproduct of matrix algebra if and only it has the $\mathrm{QWEP}^{4}$ it is naturally to study the connection between Tsirelons's problem and the existence of finite-dimensional approximations.

Let $\mathcal{H}$ be a Hilbert space such that $\mathcal{L} \mathcal{M}_{n, k} \subset \mathcal{B}(\mathcal{H})$, and let $a \in \mathcal{L} \mathcal{M}_{n, k}$. If $p_{n}$ is a sequence of projections onto finite-dimensional subspaces of $\mathcal{H}$, then we have that the sequence $p_{n} a p_{n}$ converges weakly to $a$. Now consider the minimal tensor product of $\mathcal{L} \mathcal{M}_{n, k}$ with itself, and note that $\mathcal{L} \mathcal{M}_{n, k} \otimes_{\text {min }} \mathcal{L} \mathcal{M}_{n, k} \subset \mathcal{B}(\mathcal{H} \otimes \mathcal{H})$. The sequence $p_{n} a p_{n} \otimes \mathbb{I}_{\mathcal{H}}$ commutes still with all elements of the form $\mathbb{I}_{\mathcal{H}} \otimes p_{n} b p_{n}, a, b \in \mathcal{L} \mathcal{M}_{n, k}$. Now let $\omega$ be a state on $\mathcal{B}(\mathcal{H} \otimes \mathcal{H})$. Its restriction $\omega\left(p_{n} \otimes p_{n} . p_{n} \otimes p_{n}\right)$ onto the finite-dimensional $\mathrm{C}^{*}$-algebra $\mathcal{B}\left(p_{n} \mathcal{H} \otimes p_{n} \mathcal{H}\right)$ is again a state. Hence we have that for any pair of quantum representations of $\mathcal{L} \mathcal{M}_{n, k}$ in tensor product form given by a pair of completely positive unital maps $\phi, \phi^{\prime}$ and any $\varepsilon>0$, there is a natural number $n$, a density matrix $\rho$ on $\mathbb{C}^{n} \otimes \mathbb{C}^{n}$ and a pair of completely positive and unital maps $\phi_{n}, \phi_{n}^{\prime}$ mapping $\mathcal{L} \mathcal{M}_{n, k}$ into $\mathcal{B}\left(\mathbb{C}^{n}\right)$ such that

$$
\left|\omega\left(\phi(a) \otimes \phi^{\prime}(b)\right)-\operatorname{Tr} \rho \phi_{n}(a) \otimes \phi_{n}^{\prime}(b)\right| \leq \varepsilon
$$

for all $a, b \in \mathcal{L M}_{n, k}$ with norm bounded by one. Assuming that Kirchberg's QWEP conjecture has a positive answer we have

$$
\mathcal{L M}_{n, k} \otimes_{\min } \mathcal{L M}_{n, k} \simeq \mathcal{L} \mathcal{M}_{n, k} \otimes_{c} \mathcal{L} \mathcal{M}_{n, k}
$$

and obtain that all bipartite correlation tables can be approximately written as correlation table originating from finite-dimensional quantum systems. For the converse, note that the minimal tensor product cone is defined to be the set of elements $z \in M_{n} \otimes \mathcal{L} \mathcal{M}_{n, k} \otimes \mathcal{L} \mathcal{M}_{n, k}$ such that the image under all maps $\phi \otimes \phi^{\prime}$, where $\phi$ and $\phi^{\prime}$ map $\mathcal{L} \mathcal{M}_{n, k}$ into $M_{n}$ completely positive and unital, is positive. If now every element of $\left(M_{n} \otimes \mathcal{L} \mathcal{M}_{n, k} \otimes_{c} \mathcal{L M}_{n, k}\right)_{+}$ admits an approximation of the form (4.5), then it follows that $\mathcal{L} \mathcal{M}_{n, k} \otimes_{c} \mathcal{L M}_{n, k} \simeq$ $\mathcal{L M}_{n, k} \otimes_{\text {min }} \mathcal{L M}_{n, k}$.

The operator system $\mathcal{L M}_{n, k}$ models the case of measurements on some arbitrary quantum system. However, from physical considerations we might have some prior information on the operator system modeling the quantum system which we want to study. It is hence natural to ask for finite-dimensional approximations for a specific operator system and the conditions for their existence. This is the topic of the next chapter.

\footnotetext{
${ }^{4}$ Note that this result can be improved. Brown has shown that a von Neumann algebra of type $I I_{1}$ is embeddable into the ultrapower of the hyperfinite type $I I_{1}$ factor if and only if it has the WEP with respect to some dense subalgebra, [12].
} 


\section{Tensor products and finite-dimensional approximations}

\subsection{An operator system perspective on QWEP}

Introduction. Let $\left\{x_{1}, \ldots, x_{k}\right\}$ be some set of defining observables of a physical systems, acting on some Hilbert space $\mathcal{H}$, where $k$ can be finite as well as infinite. These could be POVM elements, or some function of non-self-adjoint operators like in the case of bosonic or fermionic systems. Let $S$ be the operator system generated by these observables. In general, this object is much "smaller" than the algebra generated by the same set, and hence might be easier to analyze. If we want to understand the notion of joint systems where one constituent is the physical system described by the set of observables $\left\{x_{1}, \ldots, x_{k}\right\}$, a natural object to study is $S \otimes_{c} S^{\prime}$, where $S^{\prime}$ is the set of operators on $\mathcal{H}$ commuting with all our observables. This set is a $\mathrm{C}^{*}$-algebra (even a von Neumann algebra), and hence we have $S \otimes_{c} S^{\prime} \cong S \otimes_{\max } S^{\prime}$. The consideration of this section are based on the study of the joint system $S \otimes_{\max } S^{\prime}$. We note that the inclusion of $S$ into the von Neumann algebra $S^{\prime \prime}$, the double commutant of $S$, yields a complete order embedding.

From the definition of the double commutant expectation property, which is the equivalent of the weak expectation property for operator systems, we would expect that the embedding of $S$ into some QWEP von Neumann algebra preserves "locality constraints", that is it is stable under the commuting tensor product for operator systems. This motivates the following definition.

Definition 5.1.1. Let $S$ be an operator system. We say that $S$ is completely positive complemented by a $Q W E P$ von Neumann algebra, abbreviated by $Q W E P C P$, if there exists a complete order embedding $S \subset \mathcal{N}$ into a von Neumann algebra which is the $\mathrm{C}^{*}$-quotient of a $\mathrm{C}^{*}$-algebra with the weak expectation property, such that for any operator system $V$ we have a complete order embedding

$$
S \otimes_{c} V \subset \mathcal{N} \otimes_{c} V
$$


Since $\mathcal{B}(\mathcal{H})$ for any Hilbert space $\mathcal{H}$ is QWEP, we see that any operator system with the DCEP is also QWEPCP. In analyzing the class of QWEPCP operator systems, it is useful to recall a result of Junge, nicely characterizing the class of von Neumann algebras which are QWEP. As already explained in the introductory section on the QWEP conjecture, a $\mathrm{C}^{*}$-algebra $\mathcal{A}$ which is the quotient of a $\mathrm{C}^{*}$-algebra with the weak expectation property admits an inclusion $\mathcal{A} \subset \mathcal{A}^{* *} \subset \mathcal{B}(\mathcal{H})^{* *}$ (not necessarily unital) such that there is a normal conditional expectation $E: \mathcal{B}(\mathcal{H})^{* *} \rightarrow \mathcal{A}^{* *}$. If $\mathcal{A}$ is a von Neumann algebra then the conditional expectation can be taken to project onto $\mathcal{A}$ itself. This leads to a little lemma characterizing those operator systems which embed into a QWEP von Neumann algebra in a "tensor" stable way, i.e. are QWEPCP.

Lemma 5.1.1. The following properties of an operator system $S$ are equivalent.

(i) $S$ is completely positive complemented by a QWEP von Neumann algebra.

(ii) There exists a complete order embedding $S \subset \mathcal{N}$ into a von Neumann algebra which is $Q W E P$ such that for all $C^{*}$-algebras $\mathcal{A}$ it follows that

$$
S \otimes_{\max } \mathcal{A} \subset \mathcal{N} \otimes_{\max } \mathcal{A}
$$

(iii) There exists an complete order embedding $S \subset \mathcal{B}(\mathcal{H})^{* *}$ such that for all $C^{*}$-algebras $\mathcal{A}$ it follows that

$$
S \otimes_{\max } \mathcal{A} \subset \mathcal{B}(\mathcal{H})^{* *} \otimes_{\max } \mathcal{A}
$$

(iv) There exists an complete order embedding $S \subset \mathcal{B}(\mathcal{H})^{* *}$ such that for all operator systems $V$ it follows that

$$
S \otimes_{c} V \subset \mathcal{B}(\mathcal{H})^{* *} \otimes_{c} V
$$

Proof. ad (i) $\Rightarrow$ (ii) follows since the maximal and the commuting tensor product coincide if one of the factors is a $\mathrm{C}^{*}$-algebra.

ad (ii) $\Rightarrow$ (iii) [similar to the implication (5) to (4) in Thm. 7.1 in [52]].

According to Lemma 2.1 in [44] there exists an inclusion $\mathcal{N} \subset \mathcal{B}(\mathcal{H})^{* *}$ as a subalgebra such that $\mathcal{N}$ is equal to the range of a normal conditional expectation $E: \mathcal{B}(\mathcal{H})^{* *} \rightarrow \mathcal{N}$. However, the $*$-homomorphism $i: \mathcal{N} \rightarrow \mathcal{B}(\mathcal{H})^{* *}$ does not have to be unital. In that case, define $p$ to be the projection $i\left(\mathbb{I}_{\mathcal{N}}\right)$ and let $j: S \rightarrow \mathcal{N}$ be the complete order embedding. Then the composition $i \circ j: S \rightarrow \mathcal{B}(\mathcal{H})^{* *}$ is completely positive. Since $\mathcal{B}(\mathcal{H})^{* *}$ is a von Neumann algebra, we may apply the unitization lemma of Choi and Effros and find a completely positive unital and in this case injective map $\varphi: S \rightarrow \mathcal{B}(\mathcal{H})^{* *}$ with

$$
i \circ j(s)=p \varphi(s) p
$$

Now consider the completely positive map $\varphi \otimes$ id $: S \otimes_{\max } \mathcal{A} \rightarrow \mathcal{B}(\mathcal{H})^{* *} \otimes_{\max } \mathcal{A}$, and let $s \in S \otimes_{\max } \mathcal{A}$ be such $\varphi \otimes \operatorname{id}(s) \in\left(\mathcal{B}(\mathcal{H})^{* *} \otimes_{\max } \mathcal{A}\right)_{+} \cap \varphi(S) \otimes \mathcal{A}$. We have to show that $s \in\left(S \otimes_{\max } \mathcal{A}\right)_{+}$. But $E(p \varphi(s) p)=s$ and hence

$$
s=(E \otimes \mathrm{id})(p \varphi(s) p) \in\left(\mathcal{N} \otimes_{\max } \mathcal{A}\right)_{+} \cap S \otimes \mathcal{A}=\left(S \otimes_{\max } \mathcal{A}\right)_{+}
$$

from which the implication follows. 
ad (iii) $\Rightarrow$ (iv).

We have that $S \otimes_{c} V \subset S \otimes_{\max } C^{*}(V)$ and $\mathcal{B}(\mathcal{H})^{* *} \otimes_{c} V \subset \mathcal{B}(\mathcal{H})^{* *} \otimes_{\max } C^{*}(V)$. Hence we have the following chain of inclusions,

$$
S \otimes_{c} V \subset S \otimes_{\max } C^{*}(V) \subset \mathcal{B}(\mathcal{H})^{* *} \otimes_{\max } C^{*}(V) \supset \mathcal{B}(\mathcal{H})^{* *} \otimes_{c} V,
$$

which together imply $S \otimes_{c} V \subset \mathcal{B}(\mathcal{H})^{* *} \otimes_{c} V$.

ad (iv) $\Rightarrow$ (i) follows from the fact that since $\mathcal{B}(\mathcal{H})$ is injective and therefore has the WEP it follows that $\mathcal{B}(\mathcal{H})^{* *}$ is QWEP.

An operator system perspective on QWEP. The QWEPCP property can be understood in terms of the following result by studying the von Neumann algebras generated from it. We let $S$ be a general operator system and $\mathcal{A}$ a general $\mathrm{C}^{*}$-algebra. If $\mathcal{B}$ is a von Neumann algebra, and $\phi: S \rightarrow \mathcal{B}$ is a complete order embedding, we denote by $\phi(S)^{\prime \prime}$ the von Neumann subalgebra of $\mathcal{B}$ generated by the range of $\phi(S)$. Equivalently, it is the double commutant of the set $\phi(S) \subset \mathcal{B}$.

Theorem 5.1.2. Let $S$ be an operator system. Then the following are equivalent.

(i) $S$ is completely positive complemented by a QWEP von Neumann algebra.

(ii) There exist a von Neumann algebra $\mathcal{N}$ which is $Q W E P$ and a complete order embedding of $S$ into $\mathcal{N}$, such that for any $C^{*}$-algebra $\mathcal{A}$,

$$
S \otimes_{\max } \mathcal{A} \subset \mathcal{N} \otimes_{\max } \mathcal{A}
$$

is a complete order embedding.

(iii) There exists an inclusion $S \subset \mathcal{N}$ into a von Neumann algebra which is $Q W E P$, such that for every complete order embedding $\phi: S \rightarrow \mathcal{B}(\mathcal{H})$, there exists a completely positive map $\varphi: \mathcal{N} \rightarrow \phi(S)^{\prime \prime}$ with $\varphi(s)=s$ for all $s \in S$.

(iv) There exists an inclusion $S \subset \mathcal{B}$ into a $C^{*}$-algebra which is $Q W E P$, such that for every complete order embedding $\phi: S \rightarrow \mathcal{B}(\mathcal{H})$, there exists a completely positive map $\varphi: \mathcal{B} \rightarrow \phi(S)^{\prime \prime}$ with $\varphi(s)=s$ for all $s \in S$.

(v) There exist a $C^{*}$-algebra $\mathcal{A}$ which is $Q W E P$ and a closed two-sided ideal $J \subset \mathcal{A}$ such that the operator system $S \oplus J \subset \mathcal{A}$ has the double commutant expectation property (DCEP).

Proof. We achieve the above theorem by proving the implications

$$
\text { (ii) } \Rightarrow \text { (iii) } \Rightarrow \text { (iv) } \Rightarrow \text { (v) } \Rightarrow \text { (ii) . }
$$

The equivalence of (i) and (ii) follows directly from lemma 5.1.1, or equivalently, from the definition of QWEPCP itself.

ad (ii) $\Rightarrow$ (iii) [The proof is analogues to the proof of Theorem $7.3(5) \Rightarrow(4)$ in [52].]

Let $\phi: S \rightarrow \mathcal{B}(\mathcal{H})$ be a complete order embedding, and denote by $\phi(S)^{\prime}$ the commutant of the range of $\phi$. It is clearly a $\mathrm{C}^{*}$-algebra, and hence we have

$$
S \otimes_{\max } \phi(S)^{\prime} \subset \mathcal{N} \otimes_{\max } \phi(S)^{\prime} .
$$


We consider the completely positive map $\varphi: S \otimes_{\max } \phi(S)^{\prime} \rightarrow \mathcal{B}(\mathcal{H})$ given by $\varphi(s \otimes x)=s x$. Since by the well-known theorem of Arveson, see also [70], $\mathcal{B}(\mathcal{H})$ is injective, we obtain the following commuting diagram,

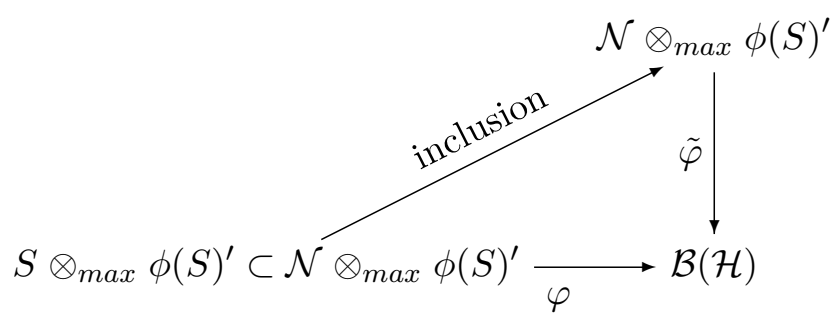

and $\tilde{\varphi}: \mathcal{N} \otimes_{\max } \phi(S)^{\prime} \rightarrow \mathcal{B}(\mathcal{H})$ is a completely positive extension of $\varphi$. In particular, its restriction $\left.\tilde{\varphi}\right|_{\phi(S)^{\prime}}$ is a $*$-homomorphism. By Choi's theorem on multiplicative domains, [70], $\tilde{\varphi}$ is a $\phi(S)^{\prime}$-bimodule map. We define $E: \mathcal{N} \rightarrow \mathcal{B}(\mathcal{H})$ as $E(a)=\tilde{\varphi}\left(a \otimes \mathbb{I}_{\mathcal{H}}\right)$. By the bimodule property, we have

$$
E(a) x=\tilde{\varphi}\left(a \otimes \mathbb{I}_{\mathcal{H}}\right) x=\tilde{\varphi}(a \otimes x)=x \tilde{\varphi}\left(a \otimes \mathbb{I}_{\mathcal{H}}\right)=x E(a)
$$

and hence $E(a) \in \phi(S)^{\prime \prime}$ for every $a \in \mathcal{N}$. Moreover, $E(s)=s$ for every $s \in S$.

ad (iii) $\Rightarrow$ (iv) is trivial.

$\operatorname{ad}(\mathrm{iv}) \Rightarrow(\mathrm{v})$

Let $\mathcal{A}$ be a $\mathrm{C}^{*}$-algebra with WEP, $J \subset \mathcal{A}$ an ideal such that $\mathcal{B} \simeq \mathcal{A} / J$. We claim that $S \oplus J$ is an operator system satisfying the DCEP property. Let $\mathcal{K}$ be a Hilbert space such that $\mathcal{A} \subset \mathcal{A}^{* *} \subset \mathcal{B}(\mathcal{K})$. Since $\mathcal{A}^{* *}$ is a von Neumann algebra, there exist a central projection $e \in \mathcal{A}^{* *}$ such that

$$
\mathcal{A}^{* *} \simeq \mathcal{A}^{* *} e \oplus \mathcal{A}^{* *}(1-e) \simeq \mathcal{B}^{* *} \oplus J^{* *} \subset \mathcal{B}(\mathcal{K})
$$

Hence, we have a complete order embedding

$$
i: S \oplus J \subset \mathcal{B} \oplus J \subset \mathcal{B}^{* *} \oplus J^{* *}
$$

Since $\mathcal{A}$ has the WEP, its bidual $\mathcal{A}^{* *}$ is QWEP, and the inclusion $i$ extends to a completely positive map $\hat{i}: \mathcal{B}(\mathcal{K})^{* *} \rightarrow \mathcal{B}^{* *} \oplus J^{* *}$, such that $\hat{i}(s \oplus k)=s \oplus k$, for $s \in S$ and $k \in J,[69]$. Moreover, if $I(S \oplus J)$ is the injective envelope of the operator system $S \oplus J$ (see the book by Paulsen [70], chapter 15), then the inclusion map $i$ also extends to a completely positive map $\tilde{i}: I(S \oplus J) \rightarrow \mathcal{B}(\mathcal{K}) \subset \mathcal{B}(\mathcal{K})^{* *}$. Let $\mathcal{H}$ be another Hilbert space, and $j: S \oplus J \rightarrow \mathcal{B}(\mathcal{H})$ be an inclusion. By assumption, there exists a completely positive map $\varphi: \mathcal{B} \rightarrow j(S)^{\prime \prime}$ which fixes $S$. Its double dual $\varphi^{* *}: \mathcal{B}^{* *} \rightarrow\left(j(S)^{\prime \prime}\right)^{* *}$ clearly does the same. Since $j(S)^{\prime \prime}$ is a von Neumann algebra, there exists a normal conditional expectation $E:\left(j(S)^{\prime \prime}\right)^{* *} \rightarrow j(S)^{\prime \prime}$ 1. By composition, we obtain a completely positive map $E \circ \varphi^{* *}: \mathcal{B}^{* *} \rightarrow j(S)^{\prime \prime}$ fixing $S$. Consider now the restriction of $j$ to the algebra $J$, and let $j(k)=v^{*} \pi(k) v$ be its Stinespring dilation, with a Hilbert space $\tilde{\mathcal{H}}$, a representation $\pi: J \rightarrow \mathcal{B}(\tilde{\mathcal{H}})$, and an isometry $v: \mathcal{H} \rightarrow \tilde{\mathcal{H}}$. Since $J$ is a $C^{*}$-algebra and $J^{* *}$ is its universal von Neumann algebra, it follows that $\pi$ can be extended to $J^{* *}$ and $\pi\left(J^{* *}\right) \subset \pi(J)^{\prime \prime}$. By the commutant lifting

\footnotetext{
${ }^{1}$ Take for example the dual map of the canonical inclusion of the predual $\left(j(S)^{\prime \prime}\right)_{*}$ into the dual $\left(j(S)^{\prime \prime}\right)^{*}$.
} 
theorem of Arveson, there exists a $*$-homomorphism $\delta: j(J)^{\prime} \rightarrow \pi(J)^{\prime}$ with $v z=\delta(z) v$. Hence, we have that

$$
v^{*} \pi(\hat{k}) v z=v^{*} \pi(\hat{k}) \delta(z) v=v^{*} \delta(z) \pi(\hat{k}) v=z v^{*} \pi(\hat{k}) v
$$

for $\hat{k} \in J^{* *}$ from which it follows that $v^{*} \pi\left(J^{* *}\right) v \subset j(J)^{\prime \prime}$. By construction, the restriction of the map $\hat{j}: J^{* *} \rightarrow v^{*} \pi\left(J^{* *}\right) v$ onto $J$ is equal to $j$ and still fixes $J$. Combining these maps, we obtain the chain

$$
I(S \oplus J) \stackrel{\tilde{i}}{\rightarrow} \mathcal{B}(\mathcal{K}) \stackrel{\hat{i}}{\rightarrow} \mathcal{B}^{* *} \oplus J^{* *} \stackrel{E \circ \varphi^{* *} \oplus \hat{j}}{\longrightarrow} j(S)^{\prime \prime} \oplus j(J)^{\prime \prime}
$$

which fixes the operator system $S \oplus J$ since every member of the chain does so. By Theorem $7.3,(2)$ in [52], the operator system $S \oplus J$ has the DCEP property.

ad $(\mathrm{v}) \Rightarrow$ (ii)

For every $\mathrm{C}^{*}$-algebra $\mathcal{B}$, we have the following chain of inclusions

$$
S \otimes_{\max } \mathcal{B} \simeq(S \oplus J / J) \otimes_{\max } \mathcal{B} \simeq \frac{(S \oplus J) \otimes_{\max } \mathcal{B}}{J \otimes_{\max } \mathcal{B}} \subset \frac{\mathcal{A} \otimes_{\max } \mathcal{B}}{J \otimes_{\max } \mathcal{B}} \simeq \mathcal{A} / J \otimes_{\max } \mathcal{B}
$$

The third isomorphism follows from [39], Theorem 3.4 and the fact that the commuting and the maximal tensor product in the operator system category agree if one of the factors is a $\mathrm{C}^{*}$-algebra. The inclusion is due to the fact that $S \oplus J$ is an operator system with the DCEP. Since $\mathcal{A}$ is QWEP, $\mathcal{A} / J$ and its bidual $(\mathcal{A} / J)^{* *}$ also are QWEP, and we have the inclusion

$$
S \otimes_{\max } \mathcal{B} \subset \mathcal{A} / J \otimes_{\max } \mathcal{B} \subset(\mathcal{A} / J)^{* *} \otimes_{\max } \mathcal{B} .
$$

Hence, $(\mathcal{A} / J)^{* *}$ is the desired von Neumann algebra with QWEP.

The last theorem indicates a strong connection between the QWEPCP and QWEP algebras. The next theorem further strengthens this view. In the algebraic case, it is known (see [69]) that a QWEP C*-algebra having the LLP satisfies the weak expectation property. If we replace $\mathrm{C}^{*}$-algebras by operator systems, and the LLP and QWEP with the corresponding notions of OSLLP and QWEPCP, we arrive at the following.

Theorem 5.1.3. Let $S$ be an operator system with the OSLLP. Then the following are equivalent.

(i) $S$ is completely positive complemented by a QWEP von Neumann algebra.

(ii) $S$ has the double commutant expectation property (DCEP).

(iii) For every complete order embedding $\iota: S \rightarrow \mathcal{B}(\mathcal{H})$, there exist a $C^{*}$-algebra $\mathcal{A}$ and a representation $\pi: \mathcal{A} \rightarrow \iota(S)^{\prime}$ with dense range such that

$$
\sum_{i} s_{i} \otimes a_{i} \rightarrow \sum_{i} \iota\left(s_{i}\right) \pi\left(a_{i}\right)
$$

extends to a completely positive map on $S \otimes_{\min } \mathcal{A}$.

Remark 5.1.4. The assumption that $S$ satisfies the OSLLP is only used in the proof of the implication (i) $\Rightarrow$ (ii). Hence, if $S$ is some arbitrary operator system, we still have the implications

$$
(\text { ii }) \Rightarrow \text { (iii) and }(i i) \Rightarrow(i) \text {, }
$$

where the second one follows from the definition of $Q W E P C P$. 
Proof. ad (i) $\Rightarrow$ (ii).

We use the characterization (iv) in Theorem 5.1.2. Let $T \subset S$ be a finite-dimensional suboperator system of $S$. Let $\mathcal{A}$ be a $\mathrm{C}^{*}$-algebra with the WEP, $J$ an ideal such that $\mathcal{B} \simeq \mathcal{A} / J$, and $\varphi: T \rightarrow \mathcal{A}$ be the lifting of the inclusion $i: T \subset S \rightarrow \mathcal{B}$. Take a Hilbert space $\mathcal{K}$ such that $\mathcal{A} \subset \mathcal{A}^{* *} \subset \mathcal{B}(\mathcal{K})$. Since $\mathcal{A}$ has the WEP, $\varphi$ extends to a completely positive map $\tilde{\varphi}_{T}: \mathcal{B}(\mathcal{K}) \rightarrow \mathcal{A}^{* *}$, fixing $T$. Composing this map with the double dual of the quotient map $\pi^{* *}: \mathcal{A}^{* *} \rightarrow \mathcal{B}^{* *}$ yields again a completely positive map fixing $T$. Let now $\phi: S \rightarrow \mathcal{B}(\mathcal{H})$ be an inclusion. By assumption, there exists a completely positive map $\theta: \mathcal{B} \rightarrow \phi(S)^{\prime \prime}$ fixing $S$. Its double dual does the same, and as in the proof of Theorem 5.1.2, we can compose it with the canonical normal conditional expectation $E$ onto $\phi(S)^{\prime \prime}{ }^{2}$. Both $\mathcal{B}(\mathcal{K})$ as well as $\phi(S)^{\prime \prime}$ are von Neumann algebras, and hence weak-*-closed. Thus, any cluster-point of the net of maps $E \circ \theta \circ \pi^{* *} \circ \tilde{\varphi}_{T}{ }^{3}$ is a completely positive map $\gamma: \mathcal{B}(\mathcal{K}) \rightarrow \phi(S)^{\prime \prime}$, fixing $S$. Moreover, the inclusion $\hat{\varphi}: S \rightarrow \mathcal{B}(\mathcal{K})$ extends to a map from the injective envelope of $S, I(S)$ to $\mathcal{B}(\mathcal{K})$. By composition, we obtain a completely positive map $\tilde{\gamma}: I(S) \rightarrow \phi(S)^{\prime \prime}$, fixing $S$. Hence $S$ has the DCEP.

ad (ii) $\Rightarrow$ (iii).

Let $\mathcal{A}$ be a $\mathrm{C}^{*}$-algebra having the LLP, $\pi: \mathcal{A} \rightarrow \iota(S)^{\prime}$ a map with dense range. For example, one could take $\mathcal{A}=C^{*}\left(\mathbb{F}_{\infty}\right)$ and $\pi$ the quotient map ${ }^{4}$. It follows that the completely positive maps $\iota$ and $\pi$ have commuting ranges and hence

$$
\iota \otimes \pi: \sum_{i} s_{i} \otimes a_{i} \mapsto \sum_{i} \iota\left(s_{i}\right) \cdot \pi\left(a_{i}\right)
$$

extends to a map from $S \otimes_{c} \mathcal{A}$ to $\mathcal{B}(\mathcal{H})$. But since $S$ has the DCEP, and $\mathcal{A}$, by assumption, has LLP, which implies the OSLLP, we have due to Lemma 3.2.8 that

$$
S \otimes_{\min } \mathcal{A}=S \otimes_{c} \mathcal{A},
$$

and the map $\iota \otimes \pi$ extends to a map $\varphi$ from $S \otimes_{\min } \mathcal{A}$ to $\mathcal{B}(\mathcal{H})$ such that $\varphi\left(S \otimes \operatorname{id}_{\mathcal{A}}\right)=\iota(S)$, and $\varphi\left(\operatorname{id}_{S} \otimes \mathcal{A}\right)=\pi(\mathcal{A})$.

ad (iii) $\Rightarrow$ (ii).

Let $\mathcal{K}$ be a Hilbert space such that $S \subset \mathcal{B}(\mathcal{K})$. Since $\mathcal{B}(\mathcal{H})$ and the minimal tensor product are both injective in their respective categories, the map

$$
\varphi: S \otimes_{\min } \mathcal{A} \rightarrow \mathcal{B}(\mathcal{H})
$$

can be extended to

$$
\tilde{\varphi}: \mathcal{B}(\mathcal{K}) \otimes_{\min } \mathcal{A} \rightarrow \mathcal{B}(\mathcal{H}) .
$$

The restriction of $\tilde{\varphi}$ to the $\mathrm{C}^{*}$-algebra $\mathcal{A}$ is a $*$-homomorphism, which implies as in the proof of theorem 5.1.2, implication ad (ii) $\Rightarrow$ (iii) that the range of the map

$$
\mathcal{B}(\mathcal{K}) \ni x \rightarrow \tilde{\varphi}\left(x \otimes \mathbb{I}_{\mathcal{A}}\right)
$$

lies inside $\pi(\mathcal{A})^{\prime}=\iota(S)^{\prime \prime}$ and fixes $\iota(S)$. Hence $S$ is DCEP.

\footnotetext{
${ }^{2}$ In short, $\phi(S)^{\prime \prime}$ is a von Neumann algebra, and hence the existence follows from the inclusion of the predual into the dual.

${ }^{3}$ The net is understood with respect to the net of finite-dimensional sub-operator systems $T$ of $S$.

${ }^{4}$ We note that $\iota(S)^{\prime}$ is a $\mathrm{C}^{*}$-algebra, and hence a quotient of $C^{*}\left(\mathbb{F}_{\infty}\right)$.
} 
Application to the operator system of local measurements. We now apply our findings to the operator system $\mathcal{L M}_{n, k}$ of $n$ local measurements with $k$ outcomes each, introduced in chapter 4 . We recall that any physical situation where some quantum system is measured, using a finite number of possible measurements with a finite number of outcomes each, can be modeled as a completely positive and unital map from $\mathcal{L} \mathcal{M}_{n, k}$ into the bounded operators $\mathcal{B}(\mathcal{K})$ on some Hilbert space $\mathcal{K}$. Due to Proposition 4.1.2 (iv) we also know that the operator system $\mathcal{L} \mathcal{M}_{n, k}$ has the OSLLP. Hence, we may apply Theorem 5.1.3 to it and obtain the following corollary.

Corollary 5.1.5. Let $\mathcal{L M}_{n, k}$ be the operator system of $n$ local measurements with $k$ outcomes each, for $n, k \in \mathbb{N}$. Then the following are equivalent.

(i) $\mathcal{L M}_{n, k}$ is completely positive complemented by a $Q W E P$ von Neumann algebra.

(ii) The minimal and the commuting tensor product on the algebraic tensor product of $\mathcal{L M}_{n, k}$ with itself coincide,

$$
\mathcal{L M}_{n, k} \otimes_{\text {min }} \mathcal{L M}_{n, k} \simeq \mathcal{L} \mathcal{M}_{n, k} \otimes_{c} \mathcal{L} \mathcal{M}_{n, k}
$$

for all $n, k \in \mathbb{N}$. Consequently, Tsirelson's problem has a positive answer.

(iii) Kirchberg's QWEP conjecture has a positive answer.

Proof. ad (i) $\Rightarrow$ (ii) follows from the fact that since $\mathcal{L} \mathcal{M}_{n, k}$ has the OSLLP, Theorem 5.1.3 implies that $\mathcal{L M}_{n, k}$ also has the DCEP. Hence the assertion follows from Lemma 3.2.8. ad (ii) $\Rightarrow$ (iii) follows from Theorem 4.2.1. Now, ad (iii) $\Rightarrow$ (i) follows from the fact that Kirchberg's conjecture implies that all $\mathrm{C}^{*}$-algebras have QWEP. Indeed, let $\mathcal{I}$ be the index set

$$
\mathcal{I} \equiv\{\phi: S \rightarrow \mathcal{B}(\mathcal{H}) \mid \phi \text { complete order embedding }\}
$$

and consider the $\mathrm{C}^{*}$-algebra

$$
\Phi(\mathcal{I}) \equiv \prod_{\phi \in \mathcal{I}} \phi(S)^{\prime \prime},
$$

which by assumption is QWEP. The projection onto the element indexed by $\phi \in \mathcal{I}$ is a completely positive map $\varphi: \Phi(\mathcal{I}) \rightarrow \phi(S)^{\prime \prime}$ fixing $S$ and thus proving (iv) in Theorem 5.1 .2 .

These results motivates the study of operator systems which are QWEPCP. In particular, it is interesting to ask for physical conditions such that the operator systems in question do satisfy any of the requirements of Theorems 5.1.2 or 5.1.3. This is the subject of the next section.

\subsection{Tsirelson's problem revisited}

Operator systems modeling physical systems. As already outlined in the introduction, a quantum system is often defined by some abstract algebraic relations between generators. These generators can be either identified with annihilation and creation operators, as in the case of bosonic or fermionic systems, or with some set of POVM elements corresponding to physical measurements. The second case is often assumed in quantum statistical 
mechanics, were the standing assumption is that not all quantities of a quantum system can be observed, but only a finite set of macroscopic quantities. Turning to quantum field theory, the basic objects are so-called Wightman fields. These are operator-valued distributions admitting an action of the Lorentz group, which relates to an action of some automorphism on the algebra of observables. It is also usually assumed that the action of these automorphisms are unitarily implemented. In all cases, the ${ }^{*}$-algebra of observables is then defined by taking arbitrary products and linear combinations, as well as adjoints, of the basic set of generators. A concrete representation is then a $*$-homomorphism into the set of bounded (or also possibly unbounded) operators on some Hilbert space. For precise formulations we refer the reader to the books of Haag [35] and Bratelli and Robinson, [68]. It is only important that by a quantum system we mean some basic set of generators, obeying certain algebraic relations and ultimately being represented as (bounded) operators on some Hilbert space.

For any of these quantum systems, let us abbreviate the linear hull of its generators by $S$. Since in any case, the basic physical observables are given by elements in $S$, we conclude that $S$ admits a meaningful notion of positivity and a unit, and hence we can define physical states as linear positive and normalized functionals on $S$. The assumptions that the cone is closed and the stability under tensoring with quantum systems with a finite number of degrees of freedom, already outlined in chapter 3, leads to the statement that $S$ is in fact an operator system. A representation of these basic physical quantities is then a completely positive and unital map into the algebra of bounded operators $\mathcal{B}(\mathcal{H})$ on some Hilbert space. Moreover, we demand that any abstract physical state, i.e. a completely positive map $\psi$ of $S$ into a matrix algebra, is also a state in the Hilbert space sense, i.e. we demand that $\psi$ admits an extension to all of $\mathcal{B}(\mathcal{H})$. Hence, for us, a "concrete quantum system" is modeled by a complete order embedding $\phi$ of the operator system of generators $S$ into $\mathcal{B}(\mathcal{H})$. The von Neumann algebra of observables is then given by the double commutant $\phi(S)^{\prime \prime}$, or equivalently by the weak closure of the algebra generated by $\phi(S)$ on $\mathcal{H}$.

Our goal is to find "nice" finite-dimensional approximations of physical quantities. That is, we would like to approximately express all physical "accessible quantities" by models only involving quantum systems with a finite number of degrees of freedom. Equivalently, we want to conclude that up to an arbitrary small error $\varepsilon$, we cannot distinguish between physical "observations" performed on $\phi(S)$ and those being described by actions on finitedimensional Hilbert spaces. Of course, we can in general not hope to exactly represent $S$ on some finite-dimensional Hilbert space, but an exact embedding is also not necessary, as physically, there is no difference between an exact embedding and an "embedding up to" some error parameter $\varepsilon$. However, this approximate embedding should still respect the "locality constraint", that is operators commuting with $\phi(S)$ should, under the image of the approximate embedding, still commute with $\phi(S)$. This motivated the definition of QWEPCP. This section studies the physical implications of this property as well as of the double commutant expectation property.

In particular, we are interested in the bipartite scenario, meaning that we consider correlation between $\phi(S)$ and some other von Neumann algebra of observables $\mathcal{A}$. This is a well-studied setup in axiomatic quantum field theory.

The nuclearity property of Buchholz and Wichmann. We need to introduce some of the basic concepts. We use the term local algebras for the net of von Neumann algebras asso- 
ciated to bounded space time regions. Here, $\mathcal{A}(\mathcal{O})$ denotes the von Neumann algebra of observables corresponding to actions in the open and bounded space time region $\mathcal{O}$. These algebras are supposed to be represented on some common Hilbert space $\mathcal{H}$. There is a special operator on $\mathcal{H}$, called the Hamiltonian $H$ of the quantum system in question, which is the generator of time translations. Moreover, there exists a unique vector $|\Omega\rangle \in \mathcal{H}$, called the vacuum state, which is the eigenstate of $H$ corresponding to the eigenvalue 0 , and which is cyclic and separating for all local algebras. For the precise mathematical requirements on the net of local algebras and the Hamiltonian $H$, see for example [35].

Also in this context, we are interested in the correlations between two independent parties Alice and Bob. This situation is modeled by considering observables $A \in \mathcal{A}\left(\mathcal{O}_{1}\right)$ on Alice' side and observables $B \in \mathcal{A}\left(\mathcal{O}_{2}\right)^{\prime}$, where the regions $\mathcal{O}_{1}$ and $\mathcal{O}_{2}$ are chosen such that $\mathcal{O}_{1}$ plus its boundary lies in the interior of $\mathcal{O}_{2}$. It follows from the axioms of algebraic quantum field theory that $A$ and $B$ commute. We are interested in the correlations with respect to the vacuum state,

$$
\langle\Omega \mid A B \Omega\rangle \text {. }
$$

Using some facts about analytic functions, Buchholz and co-workers in [13] showed that there is a continuous function $f: \mathbb{R} \rightarrow \mathbb{R}$ such that these bipartite correlations can be written as (for precise constraints see [13])

$$
\langle\Omega \mid A B \Omega\rangle=\langle\Omega \mid A f(H) B \Omega\rangle+\langle\Omega \mid B f(H) A \Omega\rangle .
$$

We note that is crucial that $|\Omega\rangle$ is invariant with respect to time translations. Buchholz and Wichmann introduced the nuclearity condition as a notion of restricting the possible number degrees of freedom in some bounded region of space and time. We use a formulation due to Longo, see [13]. Consider a fixed bounded region $\mathcal{O}$ in space and time. Then we demand that for each $\beta>0$ each member of the family of maps

$$
T: \mathcal{A}(\mathcal{O}) \rightarrow \mathcal{H}, \quad T(a)=e^{-\beta H} a|\Omega\rangle
$$

is nuclear. That is, there are sequences of vectors $\left|\xi_{i}\right\rangle \in \mathcal{H}$ and linear functionals $\tau_{i}$ on $\mathcal{A}(\mathcal{O})$ such that

$$
T(a)=\sum_{i} \tau_{i}(a)\left|\xi_{i}\right\rangle
$$

and moreover,

$$
\sum_{i}\left\|\tau_{i}\right\|\left\|\xi_{i}\right\|<\infty
$$

In [13] it was then shown that the maps $T$ with the operator $e^{-\beta H}$ replaced by $f(H)$ are still nuclear. Using equation (5.1), it can be inferred that the mapping $\mathcal{A}\left(\mathcal{O}_{1}\right) \otimes_{\min } \mathcal{A}\left(\mathcal{O}_{2}\right)^{\prime} \rightarrow$ $\mathcal{B}(\mathcal{H})$ is continuous. Using ideas similar to the proof of theorem 5.1.3, this then leads to the statement that all local algebras are hyperfinite.

Existence of finite-dimensional approximations. If we try to apply similar considerations to some operator system of generators, we note that the product $f(H) \cdot s$ is not defined in the category of operator systems. Instead, we might consider the action of some completely positive map $\psi$ on $S$. This leads to the study of maps of the form

$$
T: S \rightarrow \mathcal{A}^{*}, \quad T(s)(a)=\omega(\psi(s) \otimes a) .
$$


The natural analog of nuclearity as defined in equation (5.2) then is to demand that these maps factor completely positive through matrix algebras. This motivates the following definition.

Definition 5.2.1. Let $S$ be an operator system. We say that $S$ admits sufficiently many finite-dimensional approximations, if for all $\mathrm{C}^{*}$-algebras $\mathcal{A}$ with the LLP, $n \in \mathbb{N}$ and any state $\omega$ on $M_{n}(S) \otimes_{\max } M_{n}(\mathcal{A})$, there exist for any $\varepsilon>0$ two completely positive maps $\psi: S \rightarrow M_{m(\varepsilon)}, \varphi: \mathcal{A} \rightarrow M_{m(\varepsilon)}$ and a unit vector $|\Omega\rangle$ in $\mathbb{C}^{n m(\varepsilon)} \otimes \mathbb{C}^{n m(\varepsilon)}$ such that

$$
\left|\omega\left(\sum_{i} s_{i} \otimes a_{i}\right)-\left\langle\Omega \mid \sum_{i} \psi\left(s_{i}\right) \otimes \varphi\left(a_{i}\right) \Omega\right\rangle\right| \leq \varepsilon,
$$

for all elements $s_{i} \in M_{n} \otimes S$ and $a_{i} \in M_{n} \otimes \mathcal{A}$ with unit norm.

We remark that by density if suffices to check the validity of the above statement for sums involving only finitely many elements. Physically, we demand that the evaluation of any bipartite state can be approximately written as physical operations preparing states on finite-dimensional quantum systems followed by the action of some maximally entangled state. Being completely positive maps into matrix algebras, these operations can be understood as the interaction with some finite-dimensional quantum system.

Proposition 5.2.1. Let $S$ be an operator system. Then the following are equivalent.

(i) The operator system has the double commutant expectation property.

(ii) The operator system $S$ admits sufficiently many finite-dimensional approximations.

Proof. ad (i) $\Rightarrow$ (ii).

If $\mathcal{A}$ is some $\mathrm{C}^{*}$-algebra which has the LLP, it also has the OSLLP, and by lemma 3.2.8 we have

$$
S \otimes_{\min } \mathcal{A} \simeq S \otimes_{\max } \mathcal{A} .
$$

Consider some faithful (complete order) embedding $S \subset \mathcal{B}(\mathcal{H}), \mathcal{A} \subset \mathcal{B}(\mathcal{K})$. Since $S \otimes_{\text {min }} \mathcal{A} \subset$ $\mathcal{B}(\mathcal{H} \otimes \mathcal{K})$, any completely positive map $\tilde{\omega}: S \otimes_{\max } \mathcal{A} \rightarrow M_{n}$ extends to a completely positive mapping of $\mathcal{B}(\mathcal{H} \otimes \mathcal{K})$ into $M_{m}$. Let $\omega$ be the corresponding state on $\mathcal{B}\left(\mathbb{C}^{n} \otimes \mathcal{H} \otimes \mathcal{K}\right)$. For any $\varepsilon>0$ there exist compressions $p_{m}, q_{m}$ onto finite-dimensional subspaces of dimension equal to $m$ such that

$$
\left|\omega(s \otimes a)-\omega\left(\left(\mathbb{I}_{n} \otimes p_{m}\right) s\left(\mathbb{I}_{n} \otimes p_{m}\right) \otimes\left(\mathbb{I}_{n} \otimes q_{m}\right) a\left(\mathbb{I}_{n} \otimes q_{m}\right)\right)\right| \leq \varepsilon,
$$

for elements $s \in M_{n} \otimes \mathcal{B}(\mathcal{H})$ and $a \in \mathcal{M}_{n} \otimes \mathcal{B}(\mathcal{K})$. The state $\omega$ restricted to these finitedimensional subspaces, is apart from some rescaling, given by a density matrix $\rho \in M_{n^{2} m^{2}}$. But any such state on a joint finite-dimensional quantum system can be written as a pure state on some tensor product Hilbert space of larger dimension, say $\mathbb{C}^{n m^{\prime}} \otimes \mathbb{C}^{n m^{\prime}}$, followed by the actions of local quantum channels. The compositions of the compressions $p_{m}, q_{m}$ with these quantum channels give the completely positive maps $\psi$ and $\varphi$.

ad (ii) $\Rightarrow$ (i).

Due to the work of Kavruk et. al. [52], this amounts to prove

$$
S \otimes_{\min } C^{*}\left(\mathbb{F}_{\infty}\right)=S \otimes_{\max } C^{*}\left(\mathbb{F}_{\infty}\right) .
$$


Since the identity map from $S \otimes_{\max } C^{*}\left(\mathbb{F}_{\infty}\right)$ to $S \otimes_{\min } C^{*}\left(\mathbb{F}_{\infty}\right)$ is automatically completely positive, it suffices to show that any completely positive unital map $\tilde{\omega}: S \otimes_{\max } C^{*}\left(\mathbb{F}_{\infty}\right) \rightarrow$ $M_{n}$ can be approximated arbitrarily well by some completely positive map on $S \otimes_{\min } C^{*}\left(\mathbb{F}_{\infty}\right)$. Since then, if we would assume $x \in\left(M_{n}(S) \otimes_{\min } C^{*}\left(\mathbb{F}_{\infty}\right)\right)_{+}$but not in $\left(M_{n}(S) \otimes_{\max } C^{*}\left(\mathbb{F}_{\infty}\right)\right)_{+}$, the completely positive map separating $x$ from the cone $\left(M_{n}(S) \otimes_{\max } C^{*}\left(\mathbb{F}_{\infty}\right)\right)_{+}$would lead to a completely positive map on $S \otimes_{\min } C^{*}\left(\mathbb{F}_{\infty}\right)$, also separating $x$ from the cone $\left(M_{n}(S) \otimes_{\min } C^{*}\left(\mathbb{F}_{\infty}\right)\right)_{+}$, which gives a contradiction. Let $\omega$ be the state on $S \otimes_{\max } C^{*}\left(\mathbb{F}_{\infty}\right)$ such that $\omega(x)<0$. Choosing $\varepsilon$ sufficiently small, we find approximations $\psi: S \rightarrow M_{m}$, $\varphi: C^{*}\left(\mathbb{F}_{\infty}\right) \rightarrow M_{m}$, and some vector $|\Omega\rangle$ with

$$
\langle\Omega \mid \psi \otimes \varphi(x) \Omega\rangle<0 .
$$

The assertion now follows from the fact that the tensor product of completely positive maps into matrix algebras is a completely positive map on $S \otimes_{\min } C^{*}\left(\mathbb{F}_{\infty}\right)$.

We now turn to the study of operator systems $S$ which are QWEPCP. Theorem 5.1.2 implies that there exists a $\mathrm{C}^{*}$-ideal $J$ such that $S \oplus J$ has the DCEP. We denote by $q$ : $S \oplus J \rightarrow S$ the canonical quotient map, and let $\chi: C^{*}\left(\mathbb{F}_{\infty}\right) \rightarrow \phi(S)^{\prime}$ be a completely positive map with dense range, where $\phi: S \rightarrow \mathcal{B}(\mathcal{H})$ is some complete order embedding. Since the range of $\phi \circ q$ commutes with the range of $\chi$, we have that $\phi \circ q \otimes \chi: S \oplus J \otimes_{c} C^{*}\left(\mathbb{F}_{\infty}\right) \rightarrow \mathcal{B}(\mathcal{H})$ is completely positive. Since $S \oplus J$ has the DCEP, by lemma 3.2.8, we have

$$
S \oplus J \otimes_{\min } C^{*}\left(\mathbb{F}_{\infty}\right) \simeq S \oplus J \otimes_{c} C^{*}\left(\mathbb{F}_{\infty}\right),
$$

since $C^{*}\left(\mathbb{F}_{\infty}\right)$ has the LLP. Hence, there exists a completely positive map

$$
\varphi: S \oplus J \otimes_{\min } C^{*}\left(\mathbb{F}_{\infty}\right) \rightarrow \mathcal{B}(\mathcal{H})
$$

such that for every (finite) collection of elements $s_{i} \in S, a_{i} \in \mathcal{A} \subset \phi(S)^{\prime}$ (where $\mathcal{A}=$ $\left.\chi\left(C^{*}\left(\mathbb{F}_{\infty}\right)\right)\right)$ there exists elements $t_{i} \in S \oplus J, b_{i} \in C^{*}\left(\mathbb{F}_{\infty}\right)$ such that

$$
\varphi\left(\sum_{i} t_{i} \otimes b_{i}\right)=\sum_{i} \phi\left(s_{i}\right) \cdot a_{i} .
$$

However, this assignment is in general not invertible. After embedding $S \oplus J \otimes_{\min } C^{*}\left(\mathbb{F}_{\infty}\right)$ into $\mathcal{B}\left(\mathcal{K}_{1} \otimes \mathcal{K}_{2}\right)$, for suitable Hilbert spaces $\mathcal{K}_{1,2}$, we may extend the map $\varphi$ also to $\mathcal{B}\left(\mathcal{K}_{1} \otimes\right.$ $\left.\mathcal{K}_{2}\right)$. Hence any state $\omega$ on $\mathcal{B}(\mathcal{H})$ may be also extended to a state on $\mathcal{B}\left(\mathcal{K}_{1} \otimes \mathcal{K}_{2}\right)$, by defining $\tilde{\omega}=\omega \circ \varphi$ where $\varphi$ denotes also the extension of itself to $\mathcal{B}\left(\mathcal{K}_{1} \otimes \mathcal{K}_{2}\right)$. Applying the same reasoning as in the proof of implication (i) to (ii) of the foregoing proposition, we then have the following corollary.

Corollary 5.2.2. Suppose that an operator system $S$ is completely positive complemented by a $Q W E P$ von Neumann algebra. Then for any complete order embedding $\phi: S \rightarrow \mathcal{B}(\mathcal{H})$, any state on $\mathcal{B}(\mathcal{H})$, and any finite collections $s_{i} \in S$ and elements $a_{i}$ in a dense subalgebra of $\phi(S)^{\prime}$, there are for for any $\varepsilon>0$ finite-dimensional Hilbert spaces $\mathcal{K}_{1}, \mathcal{K}_{2}$, a pure state $|\Omega\rangle \in \mathcal{K}_{1} \otimes \mathcal{K}_{2}$, and elements $t_{i} \in \mathcal{B}\left(\mathcal{K}_{1}\right), b_{i} \in \mathcal{B}\left(\mathcal{K}_{2}\right)$ such that

$$
\left|\omega\left(\sum_{i} \phi\left(s_{i}\right) \otimes a_{i}\right)-\left\langle\Omega \mid \sum_{i} t_{i} \otimes b_{i} \Omega\right\rangle\right| \leq \varepsilon .
$$

Moreover, this property is tensor stable, that is it also holds after replacing $S$ with $M_{n}(S)$ and the state $\omega$ with some state on $\mathcal{B}\left(\mathbb{C}^{n} \otimes \mathcal{H}\right)$. 
By theorem 5.1.2, the validness of the QWEP conjecture implies that any operator system $S$ is QWEPCP. The above proposition then asserts that in this case, there is no physical difference between a finite or infinite number of degrees of freedom if we look at bipartite scenarios. 


\section{Ultraproducts of operator systems and finite-dimensional approximations}

Ultraproducts of Banach spaces. As a startup, we recall the basic construction for ultraproducts of Banach spaces. The theory of ultraproducts of Banach spaces was developed in the seventies of the last century, mainly initiated by Dacunha-Castelle and Krivine [18]. It now became a standard tool in the local theory of Banach spaces, that is the study of properties of their finite-dimensional subspaces. As a basic "meta-rule", if every finite-dimensional subspace has some approximation property, then the ultraproduct of these subspaces possesses the corresponding exact property and the starting Banach space embeds as a subspace into this construction. For more information see the excellent monograph by Heinrich, [42].

If $X_{\alpha}, \alpha \in \mathcal{I}$ is a sequence of Banach spaces, and $\mathcal{U}$ some ultrafilter on the index set $\mathcal{I}$, then we denote by $\left(X_{\alpha}\right)_{\mathcal{U}}$ the quotient of $\prod_{\alpha} X_{\alpha}$ by the subspace

$$
\mathcal{K}=\left\{\left(x_{\alpha}\right) \in \prod_{\alpha} X_{\alpha}: \lim _{\mathcal{U}}\left\|x_{\alpha}\right\|=0\right\} .
$$

We now consider dual spaces. If $X_{\alpha}^{*}$ denotes the dual space of $X_{\alpha}$, that is the space of all bounded $(\mathbb{C})$-linear functionals on $X_{\alpha}$, then we can define a map

$$
\mathcal{J}:\left(X_{\alpha}^{*}\right)_{\mathcal{U}} \rightarrow\left(X_{\alpha}\right)_{\mathcal{U}}^{*}, \quad \mathcal{J}\left(x_{\alpha}\right)\left(f_{\alpha}\right)=\lim _{\mathcal{U}} x_{\alpha}\left(f_{\alpha}\right)
$$

where $\left(f_{\alpha}\right)$ is any representing sequence of some element $f \in\left(X_{\alpha}^{*}\right)_{\mathcal{U}}$. We have that the image of $\mathcal{J}$ is weak-* dense, and moreover the image of the unit ball of $\left(X_{\alpha}^{*}\right)_{\mathcal{U}}$ is weak-* dense in the unit ball of $\left(X_{\alpha}\right)_{\mathcal{U}}^{*}$.

As already stated, the notion of ultraproducts is useful in the study of approximate properties of finite-dimensional subspaces of some Banach space. The following is an illustration of this technique. It is taken directly from [42]. We first define the notion of E-isomorphisms. Let $E, F$ be two Banach spaces, and let $T: F \rightarrow E$ be a linear and 
invertible map. Then $T$ is called an $\varepsilon$-isomorphism, if $\|T\| \leq 1+\varepsilon$ and $\left\|T^{-1}\right\| \leq 1+\varepsilon$, or equivalently, if we have for all $x \in F$

$$
(1+\varepsilon)^{-1}\|x\| \leq\|T x\| \leq(1+\varepsilon)\|x\| .
$$

If such a map exists, we say that $E$ and $F$ are $\varepsilon$-isomorphic. The structure analysis of Banach spaces partly goes along the lines of finding a collection of "nice model spaces" $\left(E_{i}\right)$ such that for each finite-dimensional subspace $M \subset F$ we have an $\varepsilon$-isomorphism into one of the $E_{i}$ 's. Ultraproducts are useful in making this notion precise. We briefly recall that $F$ embeds in $E$ if $F$ is isometrically isomorphic to a subspace of $E$.

Theorem 6.0.3 (see S. Heinrich, [42]). Let $F$ be a Banach space, and $\mathcal{X}=\left(E_{i}\right)$ be a collection of Banach spaces. Then we have the following.

(i) Suppose that for any finite-dimensional subspace $M \subset F$ and any $\varepsilon>0$ there is an element $E_{i}$ of $\mathcal{X}$ such that $M$ and $F$ are $\varepsilon$-isomorphic. Then there is an index set $\mathcal{I}$, an ultrafilter $\mathcal{U}$ on $\mathcal{I}$, an assignment $\mathcal{I} \rightarrow \mathcal{X}$ such that for $\alpha \in \mathcal{I}$ we have $E_{\alpha} \in \mathcal{X}$ and an embedding of $F$ into the ultraproduct $\left(E_{\alpha}\right)_{\mathcal{U}}$.

(ii) Let $\mathcal{U}$ be an ultrafilter on some set indexing $\mathcal{X}$ and $\varepsilon>0$, and let $M \subset\left(E_{\alpha}\right)_{\mathcal{U}}$ be a finite-dimensional subspace of the ultraproduct of $\mathcal{X}$ along $\mathcal{U}$. Then there is a set $\mathcal{I}_{0} \in \mathcal{U}$ such that for each $\alpha \in \mathcal{I}_{0}$ there are subspaces $M_{\alpha} \subset E_{\alpha}$ such that $M$ is $\varepsilon$-isomorphic to $M_{\alpha}$.

Our goal is to apply similar techniques to operator systems. Since operator systems are $*$-AOU spaces on every matrix level, we start by considering ultraproducts of $*$-AOU spaces.

\subsection{Ultraproducts of Archimedian order unit vector spaces}

Definition. Consider a sequence of $*$-AOU spaces $\left(V_{\alpha}, V_{+_{\alpha}}, e_{\alpha}\right)$, with $\alpha \in \mathcal{I}, \mathcal{I}$ a countable index set. We define $\prod_{\alpha} V_{\alpha}$ to be the vector space of all bounded sequences indexed by $\mathcal{I}$, where the element at position $\alpha$ is chosen from $V_{\alpha}$. Equivalently, $\prod_{\alpha} V_{\alpha}$ is the direct product of $V_{\alpha}$ 's. We equip this vector space with the canonical order, i.e. we define the positive cone to be $\prod_{\alpha} V_{+\alpha}$. This ordered vector space can also be equipped with the canonical involution, setting $\left(x_{\alpha}\right)^{*}=\left(x_{\alpha}^{*}\right)$, and with an order unit.

Lemma 6.1.1. The element $e=\left(e_{\alpha}\right)_{\alpha \in \mathcal{I}}$ is an archimedian order unit for the $*$-ordered vector space $\left(\prod_{\alpha} V_{\alpha}, \prod_{\alpha} V_{+\alpha}\right)$.

Proof. The hermitian elements of $\prod_{\alpha} V_{\alpha}$ with respect to the canonical involution are the bounded sequences with $\left(x_{\alpha}\right)^{*}=\left(x_{\alpha}\right)$. Since $r=\sup _{\alpha \in \mathcal{I}}\left\|x_{\alpha}\right\|<\infty$, it follows that $r e_{\alpha} \geq x_{\alpha}$ for all $\alpha \in \mathcal{I}$. Hence $r\left(e_{\alpha}\right)-\left(x_{\alpha}\right) \in \prod_{\alpha} V_{+_{\alpha}}$, which asserts that $\left(e_{\alpha}\right)$ is an order unit. Now assume that $\left(v_{\alpha}\right)$ is a sequence such that $r\left(e_{\alpha}\right)+\left(v_{\alpha}\right) \in \prod_{\alpha} V_{+\alpha}$ for all $r>0$. Hence, we have that $r e_{\alpha}+v_{\alpha} \in V_{+_{\alpha}}$ for all $r>0$ and all $\alpha \in \mathcal{I}$. Since by assumption every $e_{\alpha}$ is an archimedian order unit, it follows $v_{\alpha} \in V_{+_{\alpha}}$ and hence, also $\left(v_{\alpha}\right) \in \prod_{\alpha} V_{+_{\alpha}}$.

We denote the resulting $*$-AOU space by $\left(\prod_{\alpha} V_{\alpha}, \prod_{\alpha} V_{+\alpha},\left(e_{\alpha}\right)\right)$, and call it the canonical direct product of the $*$-AOU spaces $\left(V_{\alpha}, V_{+\alpha}, e_{\alpha}\right)$. 
We now construct the quotient of $\left(\prod_{\alpha} V_{\alpha}, \prod_{\alpha} V_{+_{\alpha}},\left(e_{\alpha}\right)\right)$ by an ultrafilter $\mathcal{U}$. For that, let $\mathcal{U}$ be a free ultrafilter on the index set $\mathcal{I}$, and consider a sequence of states $\left(s_{\alpha}\right)$, with $s_{\alpha} \in S\left(V_{\alpha}\right)$. These sequences define functionals on $\prod_{\alpha} V_{\alpha}$ by setting

$$
\tilde{\mathcal{J}}: \prod_{\alpha} V_{\alpha}^{*} \rightarrow\left(\prod_{\alpha} V_{\alpha}\right)^{*} \quad \tilde{\mathcal{J}}\left[\left(s_{\alpha}\right)\right]\left[\left(v_{\alpha}\right)\right]=\lim _{\mathcal{U}} s_{\alpha}\left(v_{\alpha}\right) .
$$

The properties of an ultrafilter assures us that the limit is well defined, positive if $\left(v_{\alpha}\right) \in$ $\prod_{\alpha} V_{+\alpha}$ and that $\tilde{\mathcal{J}}\left[\left(s_{\alpha}\right)\right]\left[\left(e_{\alpha}\right)\right]=1$. Hence it defines a state on the direct product of the spaces $V_{\alpha}$. We denote the set of such states on $\prod_{\alpha} V_{\alpha}$ by $\left(S(V)_{\alpha}\right)_{\mathcal{U}}$.

Given a subset $\mathcal{G}$ of $\left(S(V)_{\alpha}\right)_{\mathcal{U}}$, we would like to identify the possible limit points of the expression (6.1). For that, we first have to consider the sequences $\left(v_{\alpha}\right)$ which converge to zero under the action of $\left(s_{\alpha}\right)$. We define the subspace $\mathcal{K}_{\mathcal{U}}(\mathcal{G})$ of $\prod_{\alpha} V_{\alpha}$ as the intersection of kernels of elements of $\mathcal{G}$,

$$
\mathcal{K}_{\mathcal{U}}(\mathcal{G})=\bigcap_{f \in \mathcal{G} \subset\left(S(V)_{\alpha}\right)_{\mathcal{U}}} \operatorname{ker} f
$$

In the case $\mathcal{G}=\left(S(V)_{\alpha}\right)_{\mathcal{U}}$, we simply write $\mathcal{K}_{\mathcal{U}}$. Any $\mathcal{G}$ is easily seen to be an order ideal of $\prod_{\alpha} V_{\alpha}$. That is, we have $a \in \mathcal{K}_{\mathcal{U}}(\mathcal{G})$ and $a \leq b$ for $b \in \prod_{\alpha} V_{\alpha}$ implies $b \in \mathcal{K}_{\mathcal{U}}(\mathcal{G})$. The possible limit points of (6.1) can now be identified with elements of the algebraic quotient $\prod_{\alpha} V_{\alpha} / \mathcal{K}_{\mathcal{U}}(\mathcal{G})$. The particular form of $\mathcal{K}_{\mathcal{U}}(\mathcal{G})$ gives us that this quotient is itself a $*$-AOU space.

Lemma 6.1.2. Let $\left(V_{\alpha}, V_{+\alpha}, e_{\alpha}\right)_{\alpha \in \mathcal{I}}$ be a set of $*-A O U$ spaces, and let $\mathcal{U}$ be a free ultrafilter on the index set $\mathcal{I}$. Then we have that the algebraic quotient $\prod_{\alpha} V_{\alpha} / \mathcal{K}_{\mathcal{U}}(\mathcal{G})$ with the cone

$$
\left(V_{+\alpha}\right)_{\mathcal{U}}(\mathcal{G})=\left\{\left(v_{\alpha}\right)+\mathcal{K}_{\mathcal{U}}(\mathcal{G}): \forall r>0 \exists k \in \mathcal{K}_{\mathcal{U}}(\mathcal{G}): r\left(e_{\alpha}\right)+k+\left(v_{\alpha}\right) \in \prod_{\alpha} V_{+_{\alpha}}\right\}
$$

and the order unit $\left(e_{\alpha}\right)+\mathcal{K}_{\mathcal{U}}(\mathcal{G})$ forms a $*$-AOU space.

Proof. There exist a $*$-AOU space $W$ and a non-zero positive unital map $\phi: \prod_{\alpha} V_{\alpha} \rightarrow W$ such that $\operatorname{ker} \phi=\mathcal{K}_{\mathcal{U}}(\mathcal{G})$. Hence the assertion follows from Theorem 3.22 in [72].

Definition 6.1.1. Let $\left(V_{\alpha}, V_{+\alpha}, e_{\alpha}\right)_{\alpha \in \mathcal{I}}$ be a set of $*$-AOU spaces, and let $\mathcal{U}$ be a free ultrafilter on the index set $\mathcal{I}$. Then the $*$-AOU space defined by lemma 6.1 .2 is called the ultraproduct of $\left(V_{\alpha}, V_{+\alpha}, e_{\alpha}\right)_{\alpha \in \mathcal{I}}$ along $\mathcal{U}$ and denoted by $\left(V_{\alpha}\right)_{\mathcal{U}}(\mathcal{G})$. If $\mathcal{G}=\left(S(V)_{\alpha}\right)_{\mathcal{U}}$, we simply write $\left(V_{\alpha}\right)_{\mathcal{U}}$.

Assuming that every $*$-AOU space $V_{\alpha}$ is equipped with an order norm, denoted by $\|.\|_{\alpha}$, we can also form the ultrafilter in the Banach space sense. This amounts to define the seminorm $\left\|\left(x_{\alpha}\right)\right\|_{\mathcal{U}}=\lim _{\mathcal{U}}\left\|x_{\alpha}\right\|_{\alpha}$ and then forming the quotient with respect to the subspace $\left\{\left(x_{\alpha}\right) \in \prod_{\alpha} V_{\alpha}:\left\|\left(x_{\alpha}\right)\right\|_{\mathcal{U}}=0\right\}$.

Lemma 6.1.3. Let $\left(V_{\alpha}, V_{+\alpha}, e_{\alpha}\right)_{\alpha \in \mathcal{I}}$ be a set of $*$-AOU spaces, each equipped with an order norm, and let $\mathcal{U}$ be a free ultrafilter on the index set $\mathcal{I}$. Then there is for every sequence of hermitian elements $\left(x_{\alpha}\right) \in \prod_{\alpha} V_{\alpha, h}$ a sequence of states $\left(\omega_{\alpha}\right), \omega_{\alpha} \in S\left(V_{\alpha}\right)$, such that

$$
\lim _{\mathcal{U}}\left\|x_{\alpha}\right\|_{\alpha}=\lim _{\mathcal{U}} \omega_{\alpha}\left(x_{\alpha}\right)
$$


Moreover, we have that

$$
\mathcal{K}_{\mathcal{U}}=\left\{\left(x_{\alpha}\right) \in \prod_{\alpha} V_{\alpha}:\left\|\left(x_{\alpha}\right)\right\|_{\mathcal{U}}=\lim _{\mathcal{U}}\left\|x_{\alpha}\right\|_{\alpha}=0\right\}
$$

Proof. Let $\left(x_{\alpha}\right) \in \prod_{\alpha} V_{\alpha, h}$ be a sequence of hermitian elements. Then it follows from eq. (3.1) that there exists a sequence of states $\left(\omega_{\alpha}\right), \omega_{\alpha} \in S\left(V_{\alpha}\right)$, and a real sequence $\varepsilon_{\alpha} \rightarrow 0$ such that

$$
\left\|x_{\alpha}\right\|_{\alpha}=\omega_{\alpha}\left(x_{\alpha}\right)-\varepsilon_{\alpha},
$$

and hence $\lim _{\mathcal{U}}\left\|x_{\alpha}\right\|=\lim _{\mathcal{U}} \omega_{\alpha}\left(x_{\alpha}\right)$. Every element $x_{\alpha}$ can be decomposed into a real $\Re e\left(x_{\alpha}\right) \in V_{\alpha, h}$ and imaginary part $\Im m\left(x_{\alpha}\right) \in V_{\alpha, h}$ such that $x_{\alpha}=\Re e\left(x_{\alpha}\right)+i \Im m\left(x_{\alpha}\right)$. Suppose now that $\left(x_{\alpha}\right) \in \mathcal{K}_{\mathcal{U}}$, it then follows that

$$
0=\lim _{\mathcal{U}} \omega_{\alpha}\left(x_{\alpha}\right)=\lim _{\mathcal{U}} \omega_{\alpha}\left(\Re e\left(x_{\alpha}\right)\right)+i \lim _{\mathcal{U}} \omega_{\alpha}\left(\Im m\left(x_{\alpha}\right)\right)
$$

and hence $\left(\Re e\left(x_{\alpha}\right)\right),\left(\Im m\left(x_{\alpha}\right)\right) \in \mathcal{K}_{\mathcal{U}}$. Since $\lim _{\mathcal{U}}\left\|x_{\alpha}\right\|_{\alpha} \leq \lim _{\mathcal{U}}\left\|\Re e\left(x_{\alpha}\right)\right\|_{\alpha}+\lim _{\mathcal{U}}\left\|\Im m\left(x_{\alpha}\right)\right\|_{\alpha}$, the inclusion $\mathcal{K}_{\mathcal{U}} \subset\left\{\left(x_{\alpha}\right) \in \prod_{\alpha} V_{\alpha}:\left\|\left(x_{\alpha}\right)\right\|_{\mathcal{U}}=0\right\}$ is proven. The reverse direction follows from [72], Lemma 4.2, which can be translated to $\omega_{\alpha}\left(x_{\alpha}\right) \leq\left\|x_{\alpha}\right\|_{\alpha}$ and hence $\lim _{\mathcal{U}} \omega_{\alpha}\left(x_{\alpha}\right) \leq \lim _{\mathcal{U}}\left\|x_{\alpha}\right\|_{\alpha}$.

Positive functionals on ultraproducts. We now examine the map $\tilde{\mathcal{J}}$, defined in equation (6.1). Obviously, $\tilde{\mathcal{J}}$ preserves positivity, since positive sequences give rise to positive functionals in the limit. Let ker $\tilde{\mathcal{J}}$ be the kernel of $\tilde{\mathcal{J}}$, i.e. the subspace of all sequences $\left(z_{\alpha}\right) \in \prod_{\alpha} V_{\alpha}^{*}$ such that

$$
\lim _{\mathcal{U}} z_{\alpha}\left(x_{\alpha}\right)=0
$$

for all sequences $\left(x_{\alpha}\right) \in \prod_{\alpha} V_{\alpha}$. We denote by $q_{\mathcal{J}}$ the algebraic quotient map $\prod_{\alpha} V_{\alpha}^{*} \rightarrow$ $\prod_{\alpha} V_{\alpha}^{*} / \operatorname{ker} \tilde{\mathcal{J}}$. Since $\tilde{\mathcal{J}}$ is positive, its kernel is an order ideal. Hence the image of $q_{\mathcal{J}}$ is an ordered vector space which we denote by $\left(V_{\alpha}^{*}\right)_{\mathcal{U}}$. Note that the cone is given as

$$
\left\{z \in\left(V_{\alpha}^{*}\right)_{\mathcal{U}}: \exists\left(\omega_{\alpha}\right) \in\left(V_{\alpha}^{*}\right)_{\mathcal{U}}+\operatorname{ker} \tilde{\mathcal{J}}, z=q\left(\omega_{\alpha}\right), \quad \omega_{\alpha} \in V_{+\alpha}^{\prime}, \quad \forall \alpha \in \mathcal{I}\right\} .
$$

Hence, if we set $\mathcal{J}=q_{\mathcal{J}} \circ \tilde{\mathcal{J}}$, we obtain a positive embedding $\mathcal{J}:\left(V_{\alpha}^{*}\right)_{\mathcal{U}} \rightarrow\left(V_{\alpha}\right)_{\mathcal{U}}^{*}$. The inverse direction can be formulated as follows.

Lemma 6.1.4. Let $F$ be a reflexive subspace of the ultraproduct $\left(V_{\alpha}\right)_{\mathcal{U}}$, containing the identity $\left(e_{\alpha}\right)$ and let $z$ be a positive functional on $F$. Then there exist a sequence $\left(\omega_{\alpha}\right)$ of positive functionals $\omega_{\alpha} \in V_{+}^{*}$ such that

$$
z(f)=\lim _{\mathcal{U}} \omega_{\alpha}\left(f_{\alpha}\right)
$$

for every representing sequence $\left(f_{\alpha}\right)$ of some element $f$ of the subspace $F$.

Proof. Since $F$ is closed, the triple $\left(F_{h},\left(V_{\alpha}\right)_{\mathcal{U}} \cap F_{h}, e\right)$ is an archimedian real ordered, reflexive vector space (where $F_{h}$ denotes the hermitian subspace of $F$ ). By Proposition 3.11 in $[72]$ we know that there exist a positive ( $\mathbb{R}$-linear) functional $\tilde{z}$ on $F_{h}$ such that

$$
z(f)=\tilde{z}(\Re e f)+i \tilde{z}(\Im m f) .
$$


We proceed by first finding a sequence of functionals $\left(z_{\alpha}\right)$ implementing $\tilde{z}$ and then show that we can choose them to be positive functionals on $V_{\alpha}$. For that, first note that the map $\mathcal{J}$ is also hermitian. Hence, if we denote by $F_{h}^{\prime}$ the dual space of $F_{h}$, i.e. the space of all continuous $\mathbb{R}$-linear functionals on $F_{h}$, and let $\mathrm{e}_{F_{h}}^{d}:\left(V_{\alpha}\right)_{\mathcal{U}}^{\prime} \rightarrow F_{h}^{\prime}$ be the restriction map, then we obtain a positive map

$$
\mathrm{e}_{F_{h}}^{d} \circ \mathcal{J}:\left(V_{\alpha}^{*}\right)_{\mathcal{U}} \rightarrow F_{h}^{*}, \quad\left(\omega_{\alpha}\right)+\operatorname{ker} \mathcal{J} \rightarrow \phi\left(\left(\omega_{\alpha}\right)\right) .
$$

Clearly, ker $\mathcal{J} \subset \operatorname{ker} \mathrm{e}_{F_{h}}^{d} \circ \mathcal{J}$. Hence it suffices to show that for every positive functional $\tilde{z}$ on $F_{h}$, there exist $\left(\omega_{\alpha}\right)+\operatorname{ker} \mathcal{J} \in \prod V_{+\alpha}^{*}$ such that $\tilde{z}=\mathrm{e}_{F_{h}}^{d} \circ \mathcal{J}\left(\left(\omega_{\alpha}\right)\right)$. For that, consider the adjoint map $\left(\mathrm{e}_{F_{h}}^{d} \circ \mathcal{J}\right)^{*}: F_{h}^{* *} \simeq F_{h} \rightarrow\left(\prod V_{\alpha, h}^{*}\right)^{*}$ and note that it is a positive and injective map. This follows from

$$
\left(\mathrm{e}_{F_{h}}^{d} \circ \mathcal{J}\right)^{*}=\mathcal{J}_{d} \circ \mathrm{e}_{F_{h}}
$$

where $\mathrm{e}_{F_{h}}: F_{h} \rightarrow \prod_{\alpha} V_{\alpha, h} / \mathcal{U}$ is the canonical embedding of $F$, and $\mathcal{J}_{d}: \prod_{\alpha} V_{\alpha, h} / \mathcal{U} \rightarrow$ $\left(\prod V_{\alpha, h}^{*}\right)^{*}$ is the canonical identification, i.e.

$$
\mathcal{J}_{d}\left[\left(f_{\alpha}\right)\right]\left[\left(\omega_{\alpha}\right)\right]=\lim _{\mathcal{U}} \omega_{\alpha}\left(f_{\alpha}\right) .
$$

Since $\left(\mathrm{e}_{F_{h}}^{d} \circ \mathcal{J}\right)^{*}$ is injective, the range of $\mathrm{e}_{F_{h}}^{d} \circ \mathcal{J}$ has to be all of $F_{h}^{*}$. Hence there exists for every element $\tilde{z} \in F_{h}^{*}$ an element $\left(z_{\alpha}\right) \in \prod V_{\alpha, h}^{*}$ such that $\tilde{z}=\mathrm{e}_{F_{h}}^{d} \circ \mathcal{J}\left(\left(z_{\alpha}\right)\right)$.

We are left to show that the sequence $\left(z_{\alpha}\right)$ can be chosen to consist only of positive functionals. For that, note that we can find a decomposition of $\tilde{z}$ into

$$
\left(z_{\alpha}\right)=\left(z_{\alpha}^{1}\right)-\left(z_{\alpha}^{2}\right),
$$

with $\left(z_{\alpha}^{1,2}\right) \in \prod_{\alpha} V_{+\alpha}$. This follows since for every $\alpha, V_{\alpha, h}$ is an archimedian real ordered vector space and hence we can find such a decomposition element wise. Moreover, we have due to Lemma 3.1.2 and the discussion afterwards

$$
\left\|\left(z_{\alpha}\right)\right\|=\left\|\left(z_{\alpha}^{1}\right)\right\|+\left\|\left(z_{\alpha}^{2}\right)\right\|=\lim _{\mathcal{U}}\left[z_{\alpha}^{1}\left(e_{\alpha}\right)+z_{\alpha}^{2}\left(e_{\alpha}\right)\right] .
$$

If now $\left(z_{\alpha}^{1}\right) \notin \operatorname{ker} \phi$ and $\left(z_{\alpha}^{2}\right) \in \operatorname{ker} \phi$ then the result follows due to the discussion above. The case $\left(z_{\alpha}^{1}\right) \in \operatorname{ker} \phi$ and $\left(z_{\alpha}^{2}\right) \notin \operatorname{ker} \phi$ clearly contradicts the positivity of $\tilde{z}$. Hence we are left with the case $\left(z_{\alpha}^{1,2}\right) \notin \operatorname{ker} \phi$. But then it follows that

$$
\left\|\left(z_{\alpha}\right)\right\|=\lim _{\mathcal{U}} z_{\alpha}^{1}\left(e_{\alpha}\right)+\lim _{\mathcal{U}} z_{\alpha}^{2}\left(e_{\alpha}\right)>\lim _{\mathcal{U}} z_{\alpha}^{1}\left(e_{\alpha}\right)-\lim _{\mathcal{U}} z_{\alpha}^{2}\left(e_{\alpha}\right)=\lim _{\mathcal{U}} z_{\alpha}\left(e_{\alpha}\right),
$$

which also contradicts the positivity of $\tilde{z}$, due to Lemma 3.1.2.

Remark 6.1.5. Note the proof just gives us the existence of a positive sequence implementing $z$, but this positive sequence is not obtained in a linear way from $z$. Equivalently, the map $\mathrm{e}_{F_{h}}^{d} \circ \mathcal{J}$ cannot be inverted in a positive way.

We now examine in more detail the structure of finite-dimensional subspaces of an ultraproduct of $*$-AOU spaces. As before, $\mathcal{I}$ denotes some general index set.

Proposition 6.1.6. Let $V$ be a finite-dimensional $*$-AOU space spanned by elements $\left\{x_{1}, \ldots, x_{n}\right\}$, and let $\left(W_{\alpha}\right)_{\alpha \in \mathcal{I}}$ be a sequence of $*-A O U$ spaces and $\mathcal{U}$ an ultrafilter on $\mathcal{I}$. Then there exists a positive embedding of $V=\operatorname{span}\left\{x_{1}, \ldots, x_{n}\right\}$ into $\left(W_{\alpha}\right)_{\mathcal{U}}$ if and only if there exist elements $y_{1}, \ldots y_{n}$ of $\left(W_{\alpha}\right)_{\mathcal{U}}$ such that the following holds. 
(i) If $a_{1}, \ldots a_{n}$ are complex numbers, and $\sum_{i} a_{i} \cdot x_{i} \geq 0$ then there are representing sequences $\tilde{y}_{i, \alpha}$ of $y_{i}$ with

$$
\sum_{i} a_{i} \cdot \tilde{y}_{i, \alpha} \geq 0
$$

for any $\alpha \in \mathcal{I}$.

(ii) If $a_{1}, \ldots a_{n}$ are complex numbers, and $\sum_{i} a_{i} \otimes x_{i}=e_{V}$ then there are representing sequences $\tilde{y}_{i, \alpha}$ of $y_{i}$ with

$$
\sum_{i} a_{i} \cdot \tilde{y}_{i, \alpha}=e_{\alpha}
$$

for any $\alpha \in \mathcal{I}$.

(iii) For every positive functional $\psi: V \rightarrow \mathbb{C}$, there exist a sequence $\left(\psi_{\alpha}\right)$ of positive functionals $\psi_{\alpha}: W_{\alpha} \rightarrow \mathbb{C}$ with

$$
\psi\left(x_{i}\right)=\lim _{\mathcal{U}} \psi_{\alpha}\left(\tilde{y}_{i, \alpha}\right)
$$

where $\tilde{y}_{i, \alpha}$ denotes any representing sequence of $y_{i}$.

Proof. We first prove that the properties (i)-(iii) imply that $V$ embeds into a subspace of $\left(W_{\alpha}\right)_{\mathcal{U}}$. Let $\phi: V \rightarrow\left(W_{\alpha}\right)_{\mathcal{U}}$ be the linear map defined by

$$
\phi\left(\sum_{i} a_{i} \cdot x_{i}\right)=\sum_{i} a_{i} \cdot y_{i}
$$

which by (i) is positive. By (ii), we have that $\phi$ is unital, since $\left(e_{\alpha}\right)+\mathcal{K}_{\mathcal{U}}$ is the archimedian order unit of $\left(W_{\alpha}\right)_{\mathcal{U}}$. We are left to prove that $\phi$ is an order isomorphism onto its range, that is the image of the positive cone is equal to the intersection of its range with the positive cone in $\left(W_{\alpha}\right)_{\mathcal{U}}$. Suppose not, then there exists a non-positive element $z \in V$ such that $\phi(z) \geq 0$. We may decompose $z$ as $z=\sum_{i} z_{i} x_{i}$, with complex numbers $z_{i}$. Since the cone $V_{+}$is closed, there exists due to the Krein-Milman theorem a map $\omega: V \rightarrow \mathbb{C}$ such that $\omega\left(V_{+}\right) \geq 0$ and $\omega(z)<0$. Hence $\omega$ is a positive functional on $V$. By property (iii) there exist a sequence $\left(\psi_{\alpha}\right)$ of positive maps such that

$$
0>\omega(z)=\sum_{i} \omega\left(z_{i} x_{i}\right)=\lim _{\mathcal{U}} \sum_{i} z_{i} \psi_{\alpha}\left(\tilde{y}_{i, \alpha}\right)=\lim _{\mathcal{U}} \sum_{i} z_{i} \psi_{\alpha}\left(\widetilde{\phi\left(x_{i}\right)_{\alpha}}\right)
$$

where $\widetilde{\phi\left(x_{i}\right)_{\alpha}}$ is any representing sequence of $\phi\left(x_{i}\right)$. Since the sequence $\left(\psi_{\alpha}\right)$ corresponds to a positive map from $\prod_{\alpha} W_{\alpha}$ to $\mathbb{C}$, this implies that there exist no element $k \in \mathcal{K}_{\mathcal{U}}$ such that $k+\phi(z) \in \prod_{\alpha} V_{+\alpha}$. Hence we have that $\phi(z) \nsupseteq 0$, contradicting the assumption.

For the converse direction, observe that the properties (i) and (ii) follow easily by the very definition of ultraproducts. The third property follows from Lemma 6.1.4 and the fact that $V$ is finite-dimensional and hence reflexive.

\subsection{Ultraproducts of operator systems}

Definitions. We now turn to the construction of ultraproducts of operator systems. We will do this along the lines of the previous section on ultraproducts of archimedian ordered 
vector spaces, as operator systems are $*$-AOU spaces on every matrix level. Let $\mathcal{I}$ be an index set and let $\left(V_{\alpha},\left\{C_{n}\right\}_{\alpha}, e_{\alpha}\right)_{\alpha \in \mathcal{I}}$ a sequence of operator systems. The direct product $\prod_{\alpha} V_{\alpha}$ is defined as the set of all bounded sequences, where the entry at position $\alpha$ is an element of $V_{\alpha}$. It can be equipped with a canonical matrix ordering by setting

$$
\left(x_{\alpha}\right) \in M_{n} \otimes \prod_{\alpha} V_{\alpha}:\left(x_{\alpha}\right) \geq 0 \Leftrightarrow x_{\alpha} \in C_{n, \alpha} \forall \alpha \in \mathcal{I},
$$

as every element $\sum_{i j}\left(x_{\alpha}\right)_{i j} \otimes\left|\xi_{i}\right\rangle\left\langle\xi_{j}\right|$ can be identified with the sequence $\left(\sum_{i j} x_{\alpha, i j} \otimes\left|\xi_{i}\right\rangle\left\langle\xi_{j}\right|\right)$ and vice versa. Colloquially speaking, a matrix of sequences with elements in $V_{\alpha}$ is just a sequence of matrices with entries in $V_{\alpha}$. We denote this family of matrix cones by $\left\{\prod_{\alpha} C_{n, \alpha}\right\}$. It follows as in the case of $*$-AOU spaces that $\mathbb{I}_{n} \otimes e=\left(\mathbb{1}_{n} \otimes e_{\alpha}\right)$ is an archimedian order unit for every cone $\prod_{\alpha} C_{n, \alpha}$ and hence $\left(\prod_{\alpha} V_{\alpha},\left\{\prod_{\alpha} C_{n, \alpha}\right\},\left(e_{\alpha}\right)\right)$ is an operator system, which we call the direct product operator system of the set $V_{\alpha}$.

If $\mathcal{U}$ is a free ultrafilter on the index set $\mathcal{I}$, and $T_{\alpha}: V_{\alpha} \rightarrow M_{k}$ is a set of completely positive maps, then we can define the associated map on the direct product $\left(\prod_{\alpha} V_{\alpha},\left\{\prod_{\alpha} C_{n, \alpha}\right\},\left(e_{\alpha}\right)\right)$ by setting

$$
\left(T_{\alpha}\right): \prod_{\alpha} V_{\alpha} \rightarrow M_{n}, \quad\left(T_{\alpha}\right)\left[\left(x_{\alpha}\right)\right]=\lim _{\mathcal{U}} T_{\alpha}\left(x_{\alpha}\right),
$$

where the convergence is taken within any norm on $M_{k}$. We call such maps ultrafilter $c p$. maps. If $T_{\alpha} \in C P\left(V_{\alpha}, M_{k}\right)$ for all $\alpha \in \mathcal{I}$ then the ultrafilter map $\left(T_{\alpha}\right)$ is completely positive since

$$
\sum_{i j}\left(x_{\alpha}\right)_{i j} \otimes\left|\xi_{i}\right\rangle\left\langle\xi_{j}\right| \mapsto \sum_{i j} \lim _{\mathcal{U}}\left\langle\xi_{i} \mid T_{\alpha}\left(x_{\alpha, i j}\right) \xi_{j}\right\rangle
$$

defines a positive functional on $M_{n} \otimes \prod_{\alpha} V_{\alpha}$.

Let now $\mathcal{P} \subset C P\left(\prod_{\alpha} V_{\alpha}, M_{k}\right)$, be a set of completely positive ultrafilter maps. Considering the intersection of the kernels of elements of $\mathcal{P}$,

$$
\mathcal{K}_{\mathcal{U}}(\mathcal{P})=\bigcap_{\left(T_{\alpha}\right) \in \mathcal{P}} \operatorname{ker}\left(T_{\alpha}\right)
$$

it follows that $\mathcal{K}_{\mathcal{U}}(\mathcal{P}) \subset \prod_{\alpha} V_{\alpha}$ fulfills the requirement to be called a kernel in the terminology of [52], Definition 3.2. Hence we have by Proposition 3.4 in the same paper that the algebraic quotient $\prod_{\alpha} V_{\alpha} / \mathcal{K}_{\mathcal{U}}(\mathcal{P})$ together with the set of cones

$$
\begin{aligned}
C_{n}\left(\left(V_{\alpha}\right)_{\mathcal{U}}(\mathcal{P})\right) & =\left\{\left(x_{\alpha}\right)+\mathcal{K}_{\mathcal{U}}(\mathcal{P}) \in M_{n} \otimes\left(\prod_{\alpha} V_{\alpha}\right):\right. \\
& \left.\left.\forall r>0 \exists k \in \mathcal{K}_{\mathcal{U}}(\mathcal{P})\right): r\left(e_{\alpha}\right)+k+\left(x_{\alpha}\right) \in \prod_{\alpha} C_{n, \alpha}\right\}
\end{aligned}
$$

is a quotient operator system. The archimedian order unit of $C_{n}\left(\left(\left(V_{\alpha}\right)_{\mathcal{U}}\right)(\mathcal{P})\right)$ is $\mathbb{1}_{n} \otimes\left(e_{\alpha}\right)+$ $\mathcal{K}_{\mathcal{U}}(\mathcal{P})$, where $e_{\alpha}$ is the Archimedian order unit for $V_{\alpha}$. In the following, we denote it simply by $\left(e_{\alpha}\right)_{\mathcal{U}}$.

Definition 6.2.1. Let $\mathcal{I}$ be an index set and let $\left(V_{\alpha}\right), \alpha \in \mathcal{I}$ be a sequence of operator systems, $\mathcal{U}$ an ultrafilter on $\mathcal{I}$, and $\mathcal{P}$ a set of ultrafilter cp maps. The operator system defined as being the algebraic quotient of $\prod_{\alpha} V_{\alpha}$ together with the set of matrix cones 
$C_{n}\left(\left(V_{\alpha}\right)_{\mathcal{U}}(\mathcal{P})\right.$ defined by equation (6.2) and the archimedian order unit $\left(e_{\alpha}\right)_{\mathcal{U}}$ is called the ultraproduct operator system of the sequence $\left(V_{\alpha}\right)$ along $\mathcal{U}$ with respect to $\mathcal{P}$, and denoted by $\left(V_{\alpha}\right)_{\mathcal{U}}(\mathcal{P})$. If $\mathcal{P}$ is the set of all ultrafilter cp maps, then we simply write $\left(V_{\alpha}\right)_{\mathcal{U}}$.

Note that we have chosen the same notation as in the case of $*$-AOU spaces, since from now on we only consider operator systems. We go on by listing some useful properties of ultraproduct operator systems. The following is a nice property of its matrix cones.

Proposition 6.2.1. Every ultrafilter quotient operator system $\left(V_{\alpha}\right)_{\mathcal{U}}(\mathcal{P})$ is completely order proximinial, that is

$$
\begin{array}{r}
C_{n}\left(\left(V_{\alpha}\right)_{\mathcal{U}}(\mathcal{P})\right)=\left\{\left(x_{\alpha}\right)+\mathcal{K}_{\mathcal{U}}(\mathcal{P}) \in M_{n} \otimes\left(\prod_{\alpha} V_{\alpha}\right):\right. \\
\left.\left.\exists k \in \mathcal{K}_{\mathcal{U}}(\mathcal{P})\right): k+\left(x_{\alpha}\right) \in \prod_{\alpha} C_{n, \alpha}\right\}
\end{array}
$$

Proof. Let $\left(x_{\alpha}\right)$ be an element of $C_{n}\left(\left(V_{\alpha}\right)_{\mathcal{U}}(\mathcal{P})\right)$ and let $\left(r_{\alpha}\right)$ be a sequence of positive numbers converging to zero. We have that for every $\alpha$ there exist $k_{\alpha} \in M_{n} \otimes V_{\alpha}$ such that $\left(k_{\alpha}\right) \in M_{n} \otimes \mathcal{K}_{\mathcal{U}}$ and

$$
r_{\alpha} \mathbb{1}_{n} \otimes e_{\alpha}+k_{\alpha}+x_{\alpha} \in C_{n, \alpha} .
$$

But since $r_{\alpha}$ converges to zero, the sequence $\left(r_{\alpha} \mathbb{I}_{n} \otimes e_{\alpha}\right)+\left(k_{\alpha}\right)$ is itself an element of $\mathcal{K}_{\mathcal{U}}$ and the assertion is proven.

In the following, we mainly investigate the ultraproduct $\left(V_{\alpha}\right)_{\mathcal{U}}$, although some statements carry over to the more general case.

Properties of ultraproducts of operator systems. We now investigate the mapping $\mathcal{J}$ : $\left(V_{\alpha}^{*}\right)_{\mathcal{U}} \rightarrow\left(V_{\alpha}\right)_{\mathcal{U}}^{*}$, introduced in the last section for $*$-AOU spaces, in the operator system category. As completely positive maps correspond to positive functionals on the matrix levels, the analysis is very similar. In particular, we have that every ultrafilter map $\left(T_{\alpha}\right)$, $T_{\alpha} \in C P\left(V_{\alpha}, M_{k}\right)$ can then be identified with a completely positive map from $\left(V_{\alpha}\right)_{\mathcal{U}}$ to $M_{k}$ and the canonical identification map is given by

$$
\mathcal{J}: \prod_{\alpha} C P\left(V_{\alpha}, M_{k}\right) \rightarrow C P\left(\left(V_{\alpha}\right)_{\mathcal{U}}(\mathcal{J}), M_{k}\right), \quad \mathcal{J}\left(T_{\alpha}\right)(x)=\lim _{\mathcal{U}} T_{\alpha}\left(x_{\alpha}\right)
$$

where $\left(x_{\alpha}\right)$ is any representing sequence for $x \in\left(V_{\alpha}\right)_{\mathcal{U}}$. The converse direction can be formulated in analogy to Lemma 6.1.4.

Corollary 6.2.2. Let $V$ be a reflexive subspace of an ultraproduct operator system $\left(V_{\alpha}\right)_{\mathcal{U}}$ and let $\psi: V \rightarrow M_{n}$ be a completely positive map. Then there exists a sequence $\left(\psi_{\alpha}\right)$ of completely positive maps $\psi_{\alpha}: V_{\alpha} \rightarrow M_{n}$ such that

$$
\psi(x)=\lim _{\mathcal{U}} \psi_{\alpha}\left(\tilde{x}_{\alpha}\right)
$$

for any representing sequence $\left(\tilde{x}_{\alpha}\right)$ of $x \in M_{k} \otimes V, k \in \mathbb{N}$. Moreover, if $\psi$ is also unital, then the maps $\psi_{\alpha}$ can chosen to be unital. 
Proof. Clearly it is enough to restrict ourselves to the case $k=n$. Note that an operator system is a $*$-AOU space on every matrix level. Thus, we can apply Lemma 6.1.4 to the functional $\tilde{\psi}$ on $M_{n} \otimes V \subset M_{n} \otimes\left(V_{\alpha}\right)_{\mathcal{U}}$ identified with $\psi \in C P\left(V, M_{n}\right)$ and get a representing sequence $\tilde{\psi}_{\alpha}$ of positive functionals on $M_{n} \otimes V_{\alpha}$ such that for every $x=\left(x_{i j}\right) \in M_{n} \otimes V$

$$
\sum_{i j} \psi\left(x_{i j}\right)_{i j}=\sum_{i j} \lim _{\mathcal{U}} \psi_{\alpha}\left(\tilde{x}_{i j, \alpha}\right)_{i j}
$$

for any representing sequence $\left(\tilde{x}_{\alpha}\right)$ of $x \in M_{n} \otimes V$. We have that every $\tilde{\psi}_{\alpha}$ can be identified with a completely positive map $\psi$ from $W_{\alpha}$ to $M_{n}$, and since the sets of completely positive maps from $V$ to $M_{n}$ and positive functionals on $M_{n} \otimes V$ are isomorphic to each other under the above identification, the first assertion is proven. Now assume that $\psi$ is unital. Then we have that

$$
\lim _{\mathcal{U}} \psi_{\alpha}\left(e_{\alpha}\right)=\psi\left(e_{V}\right)=\mathbb{1}_{n}
$$

We now apply a trick of Paulsen and define a new sequence of unital maps

$$
\phi_{\alpha}(x)=\frac{1}{\left\|\psi_{\alpha}\right\|} \psi_{\alpha}(x)+\omega_{\alpha}(x)\left(\mathbb{1}_{n}-\frac{1}{\left\|\psi_{\alpha}\right\|} \psi_{\alpha}\left(e_{\alpha}\right)\right)
$$

where $\omega_{\alpha}$ is some state on $V$. The factor $\frac{1}{\left\|\psi_{\alpha}\right\|}$ implies that the expression in brackets is positive, and hence $\phi_{\alpha}$ is a completely positive and unital map such that

$$
\psi(x)=\lim _{\mathcal{U}} \phi_{\alpha}\left(\tilde{x}_{\alpha}\right)
$$

for any representing sequence $\left(\tilde{x}_{\alpha}\right)$ of $x \in M_{n} \otimes V_{\alpha}$.

We note that in analogy to the case of $*$-AOU spaces, the representing sequence $\psi_{\alpha}$ is not obtained in a linear way, and certainly not by a completely positive map. We go on by studying ultraproducts of operator systems, and especially show the existence of representing sequences which have nice "local" properties, in the sense that every element in the sequence has the corresponding "global" property. For example, positive elements can be represented by positive sequences. First, let us comment on the structure of finitedimensional subspaces of ultraproducts.

Proposition 6.2.3. Let $V$ be a finite-dimensional operator system spanned by elements $\left\{x_{1}, \ldots, x_{n}\right\}$ and let $\left(W_{\alpha}\right)_{\alpha \in \mathcal{I}}$ be a sequence of operator systems (I $\mathcal{I}$ some index set) and $\mathcal{U}$ an ultrafilter on $\mathcal{I}$. Then there exists a completely order embedding of $V=\operatorname{span}\left\{x_{1}, \ldots, x_{n}\right\}$ into the ultraproduct operator system $(W)_{\mathcal{U}}$ if and only if there exist elements $y_{1}, \ldots y_{n}$ of $\prod_{\alpha} W_{\alpha} / \mathcal{U}$ such that

(i) If $a_{1}, \ldots a_{n}$ are matrices in $M_{k}$, and $\sum_{i} a_{i} \otimes x_{i} \geq 0$, then there are representing sequences $\tilde{y}_{i, \alpha}$ of $y_{i}$ with

$$
\sum_{i} a_{i} \otimes \tilde{y}_{i, \alpha} \geq 0
$$

for any $\alpha \in \mathcal{I}$. 
(ii) If $a_{1}, \ldots a_{n}$ are matrices in $M_{k}$, and $\sum_{k}\left(\sum_{i} a_{i} \otimes x_{i}\right)_{k k}=e_{V}$ (the sum of diagonal elements equals the unit element) then there are representing sequences $\tilde{y}_{i, \alpha}$ of $y_{i}$ with

$$
\sum_{k}\left(\sum_{i} a_{i} \otimes \tilde{y}_{i, \alpha}\right)_{k k}=e_{\alpha}
$$

for any $\alpha \in \mathcal{I}$.

(iii) for every completely positive map $\psi: V \rightarrow M_{m}$, and every $m \in \mathbb{N}$ there exists a sequence $\left(\psi_{\alpha}\right)$ of completely positive maps $\psi_{\alpha}: W_{\alpha} \rightarrow M_{m}$ with

$$
\psi\left(\sum_{i} a_{i} \otimes x_{i}\right)=\lim _{\mathcal{U}} \psi_{\alpha}\left(\sum_{i} a_{i} \otimes \tilde{y}_{i, \alpha}\right)
$$

for all matrices $a_{i} \in M_{k}$, and all $k \in \mathbb{N}$, where $\tilde{y}_{i, \alpha}$ denotes any representing sequence of $y_{i}$.

Proof. We first prove that the properties (i)-(iii) that $V$ embeds into a subspace of $\prod_{\alpha} W_{\alpha} / \mathcal{U}$. Let $\phi: V \rightarrow \prod_{\alpha} W_{\alpha} / \mathcal{U}$ be the linear map defined by

$$
\phi\left(\sum_{i} a_{i} \otimes x_{i}\right)=\sum_{i} a_{i} \otimes y_{i} .
$$

It follows from (i) and the definition of positive matrix cones on quotient operator systems that $\phi$ is completely positive. By (ii), we have that $\phi$ is unital, since $\left(e_{\alpha}\right)+\mathcal{K}_{\mathcal{U}}$ is the archimedian order unit of $\prod_{\alpha} W_{\alpha} / \mathcal{U}$. We are left to prove that $\phi$ is an order isomorphism onto its range, that is the image of the positive matrix cone is equal to the intersection of its range with the positive cone in $\prod_{\alpha} W_{\alpha} / \mathcal{U}$. Suppose not, then there exists a non-positive element $z=\left(z_{i j}\right) \in M_{m} \otimes V$ such that $\phi(z) \geq 0$. Since the cone $C_{m} \subset M_{m} \otimes V$ is closed, there exist due to the Krein-Milman theorem a map $\omega: M_{m} \otimes V \rightarrow \mathbb{C}$ such that $\omega\left(C_{m}\right) \geq 0$ and $\omega(z)<0$. Hence $\omega$ is a positive functional on $M_{m} \otimes V$ and we can identify it with a completely positive map $\hat{\omega}: V \rightarrow M_{m}$. By property (iii) there exist a sequence $\left(\psi_{\alpha}\right)$ of completely positive maps such that

$$
0>\omega(z)=\sum_{i j} \hat{\omega}\left(z_{i j}\right)_{i j}=\lim _{\mathcal{U}} \sum_{i j} \psi_{\alpha}\left(\widetilde{\phi\left(z_{i j}\right)_{\alpha}}\right)_{i j}
$$

where $\widetilde{\phi\left(z_{i j}\right)_{\alpha}}$ is any representing sequence of $\phi(z)$. Since the sequence $\left(\psi_{\alpha}\right)$ corresponds to a completely positive map from $\prod_{\alpha} W_{\alpha}$ to $M_{m}$, this implies that there exists no element $k \in M_{m} \otimes \mathcal{K}_{\mathcal{U}}$ such that $k+\phi(z) \in \prod_{\alpha} C_{m, \alpha}$ (where $C_{m, \alpha}$ denotes the cone of positive elements in $M_{m} \otimes W_{\alpha}$ ). Since every ultraproduct operator system is completely order proximinal, see Proposition 6.2.1, we have that $\phi(z) \nsupseteq 0$, contradicting the assumption.

For the converse direction, observe that the properties (i) and (ii) follow easily by the very definition of ultrafilter quotients and again from Proposition 6.2.1. The third property follows from Corollary 6.2.2 and the fact that $V$ is finite-dimensional and hence reflexive.

The last proposition leads to the following useful corollary. Here and in the following, if $V$ is an operator system and $y=\sum_{i j} y_{i j} \otimes\left|\xi_{i}\right\rangle\left\langle\xi_{j}\right| \in M_{n} \otimes V$, we abbreviate the sum $\sum_{k} y_{k k} \in V$ of its diagonal elements by $\operatorname{Tr}(y)$. 
Corollary 6.2.4. Let $\left(V_{\alpha}\right)_{\mathcal{U}}$ be an ultraproduct operator system and let $y \in M_{n} \otimes\left(V_{\alpha}\right)_{\mathcal{U}}$ be a positive element with the property that $\operatorname{Tr}(y)=\left(e_{\alpha}\right)_{\mathcal{U}}$. Then there exists a representing sequence $\left(\tilde{y}_{\alpha}\right)$ of $y$ such that

$$
\tilde{y}_{\alpha} \in C_{n, \alpha} \quad \text { and } \operatorname{Tr}\left(\tilde{y}_{\alpha}\right)=e_{\alpha}
$$

for all $\alpha \in \mathcal{I}$.

Proof. Let $\tilde{y}_{\alpha}$ be a suitable representing sequences of $y$, such that $\tilde{y}_{\alpha} \geq 0$ for any $\alpha \in \mathcal{I}$, which exists due to Proposition 6.2 .3 (we may take $V=\operatorname{span}\{y\}$ ). Since we can identify the positive cone $C_{k, \alpha} \subset M_{k} \otimes V_{\alpha}$ with the set of completely positive maps from $M_{k}$ into $V_{\alpha}$, we obtain a sequence of completely positive maps $\hat{y}_{\alpha} \in C P\left(M_{k}, V_{\alpha}\right)$ such that

$$
\lim _{\mathcal{U}} \psi_{\alpha} \circ \hat{y}_{\alpha}\left(\mathbb{I}_{k}\right)=\lim _{\mathcal{U}} \psi_{\alpha}\left(e_{\alpha}+\mathcal{K}_{\mathcal{U}}\right)=\lim _{\mathcal{U}} \psi_{\alpha}\left(e_{\alpha}\right)=\mathbb{I}_{m},
$$

where $\psi_{\alpha} \in C P_{u}\left(W_{\alpha}, M_{m}\right)$ is any sequence of completely positive unital maps. This follows from the fact that $\hat{y}_{\alpha}\left(\mathbb{I}_{k}\right)=\operatorname{Tr}\left(\tilde{y}_{\alpha}\right)$. We now apply again a trick of Paulsen, already used in Corollary 6.2.2 and define completely positive unital maps

$$
\hat{\phi}_{\alpha}(x)=\frac{1}{\left\|\tilde{y}_{\alpha}\right\|} \hat{y}_{\alpha}(x)+\rho(x)\left(e_{V}-\frac{1}{\left\|\tilde{y}_{\alpha}\right\|} \hat{y}_{\alpha}\left(\mathbb{I}_{k}\right)\right)
$$

where $\rho$ is any state on $M_{k}$ and $x \in M_{k}$. Such a $\hat{\phi}_{\alpha} \in C P\left(M_{k}, W_{\alpha}\right)$ corresponds to an element $\phi_{\alpha} \in C_{k, \alpha} \subset M_{k} \otimes V_{\alpha}$ such that $\operatorname{Tr}\left(\phi_{\alpha}\right)=\hat{\phi}_{\alpha}\left(\mathbb{I}_{k}\right)=e_{\alpha}$. Furthermore, we have that $\rho(x)\left(e_{V}-\frac{1}{\left\|\tilde{y}_{\alpha}\right\|} \hat{y}_{\alpha}\left(\mathbb{I}_{k}\right)\right) \in \mathcal{K}_{\mathcal{U}}$ since (6.4) holds for all sequences of completely positive unital maps $\psi_{\alpha} \in C P_{u}\left(W_{\alpha}, M_{m}\right)$. Hence, if $q$ denotes the quotient map from $\prod_{\alpha} V_{\alpha}$ onto $\left(V_{\alpha}\right)_{\mathcal{U}}$, we get that $q\left(\left(\phi_{\alpha}\right)\right)=y$.

Let us consider a specific example which is of use in the next chapter.

Example 6.2.1. Let $\mathcal{I}$ be an index set, $\mathcal{U}$ an ultrafilter on $I$, and $M_{\alpha}, \alpha \in \mathcal{I}$ a sequence of $\mathrm{C}^{*}$-algebras. We take as the set of ultrafilter maps $\mathcal{P}$ the set of all sequences of states $\left(\rho_{\alpha}\right)$, where $\rho_{\alpha}$ is a state on $M_{\alpha}$ and form the ultraproduct $\left(M_{\alpha}\right)_{\mathcal{U}}$. Since $\mathrm{C}^{*}$-algebras are also *-AOU spaces, we may apply Lemma 6.1.3 and get that

$$
\left(a_{\alpha}\right) \in \mathcal{K}_{\mathcal{U}}(\mathcal{P}) \leftrightarrow \lim _{\mathcal{U}}\left\|a_{\alpha}\right\|=0
$$

It follows that $\mathcal{K}_{\mathcal{U}}(\mathcal{P})$ is a two-sided ideal of the $\mathrm{C}^{*}$-algebra $\prod_{\alpha} M_{\alpha}$ and the ultraproduct operator system is in fact also a $\mathrm{C}^{*}$-algebra. It is easily seen that the order implied by the algebraic properties coincides with the order given by the construction of the ultraproduct operator system. It follows that for any sequence of states $\rho_{\alpha}$ the mapping

$$
\left(\rho_{\alpha}\right)_{\mathcal{U}}:\left(M_{\alpha}\right)_{\mathcal{U}} \rightarrow \mathbb{C}, \quad\left(\rho_{\alpha}\right)_{\mathcal{U}}\left(\left(x_{\alpha}\right)_{\mathcal{U}}\right)=\lim _{\mathcal{U}} \rho_{\alpha}\left(x_{\alpha}\right)
$$

is a state on the $\mathrm{C}^{*}$-algebra $\left(M_{\alpha}\right)_{\mathcal{U}}$. A particular example of the last kind is if we take $\mathcal{I}$ to be a sequence of natural numbers, $\mathcal{U}$ some ultrafilter on $\mathcal{I}$ and $M_{\alpha}$ be the sequence of matrix algebras acting on the Hilbert space $\mathbb{C}^{\alpha}$. By Corollary 6.2.2, we see that the resulting algebra should have some nice finite-dimensional approximation properties. We will exploit these in detail in the next section. We note that if $\mathcal{U}$ and $\mathcal{U}^{\prime}$ are two distinct ultrafilters on $\mathcal{I}$, then it is not clear whether the two $\mathrm{C}^{*}$-algebras constructed from them are isomorphic. In fact, this question strongly depends on the validness of the continuum hypothesis, see [25]. 
Characterization of approximate embeddings. We next recall the following simple observation. If $\phi: S \rightarrow \mathcal{A}$ is a completely positive and unital map of an operator system into a $\mathrm{C}^{*}$-algebra, then $\phi$ is an embedding if and only if every completely positive map $\psi: S \rightarrow M_{n}$ into a matrix algebra admits an extension $\tilde{\psi}: \mathcal{A} \rightarrow M_{n}$ with $\tilde{\psi}(\phi(x))=\psi(x)$. Indeed, given some element $x \in M_{n} \otimes S$ with $x \nsupseteq 0$, there is a completely positive map $\psi: S \rightarrow M_{n}$ to some matrix algebra such that $\psi(x) \nsupseteq 0$. Since by assumption, $\tilde{\psi}$ is an extension, we have that $\tilde{\psi}(\phi(x)) \nsupseteq 0$ and hence $\phi(x) \nsupseteq 0$ in $\mathcal{A}$. The converse follows from the fact that $M_{n}$ is an injective operator system. We also note that ii is sufficient to consider completely positive unital maps.

These considerations motivate the following "approximate" definition. For that, we introduce first the following shorthand notation for two linear maps $\psi, \varphi$, mapping an operator system into a matrix algebra $M_{m}$. We say $\psi$ "is approximating" $\varphi$ up to $\varepsilon>0$ and $n \in \mathbb{N}$, $\psi \approx_{(\varepsilon, n)} \varphi$, if we have

$$
\|\psi(x)-\varphi(x)\|_{M_{n m}} \leq \varepsilon\|x\|,
$$

for all elements $x \in M_{n} \otimes S$.

Definition 6.2.2. Let $S$ and $V$ be two operator systems. A unital, linear and injective map $\phi: S \rightarrow V$ is called an $(\varepsilon, n)$-order embedding, if the following two conditions hold.

(i) For every completely positive unital map $\psi: S \rightarrow M_{m}$ there is a completely positive unital map $\psi_{\varepsilon}: V \rightarrow M_{m}$ such that $\psi \approx_{(\varepsilon, n)} \psi_{\varepsilon} \circ \phi$.

(ii) For every completely positive unital map $\varphi: V \rightarrow M_{m}$ there is a completely positive unital map $\varphi_{\varepsilon}: S \rightarrow M_{m}$ such that $\varphi_{\varepsilon} \approx_{(\varepsilon, n)} \varphi \circ \phi$.

We note that if the map $\phi$ is itself completely positive, then the second condition is trivially fulfilled, by taking $\varphi \circ \phi$ itself. Under this standing assumption, take now an arbitrary positive element $s \in M_{n} \otimes \phi(S)$ in the range of $\phi$. Since $\psi_{\varepsilon}$ and $\phi$ are completely positive maps we have $\psi_{\varepsilon}(s) \geq 0$, and hence

$$
\psi\left(\phi^{-1}(s)+\varepsilon\|s\| \mathbb{I}_{n} \otimes \mathbb{I}_{S}\right)=\psi\left(\phi^{-1}(s)\right)+\varepsilon\|s\| \mathbb{I}_{n} \otimes \mathbb{I}_{m} \geq 0 .
$$

This implies that the inverse $\phi^{-1}$, computed on the range of $\phi$, satisfies

$$
\left.\phi^{-1}\right|_{\text {Ball }[\phi(S)]}+\varepsilon \mathbb{I}_{S} \geq 0,
$$

i.e. for any positive element $x \in M_{n} \otimes V$ with norm smaller ore equal than one in the range of $\phi$, the element id $\otimes \phi^{-1}(x)+\varepsilon \mathbb{I}_{n} \otimes \mathbb{I}_{S}$ is positive in $M_{n} \otimes S$.

This already suggests the use of ultraproducts in order to remove the dependence on $\varepsilon$. The following theorem is the operator system version of the corresponding result in the category of Banach spaces, as stated in the introduction. We need a slight restriction on the sequence of operator systems the ultraproduct is build up. We call a sequence of operator systems $\left(W_{\alpha}\right)_{\alpha \in \mathcal{I}}$, increasing, if for any pair $\alpha, \alpha^{\prime} \in \mathcal{I} \times \mathcal{I}$ we have that either $W_{\alpha} \subset W_{\alpha^{\prime}}$ or $W_{\alpha^{\prime}} \subset W_{\alpha}$. A particular example is the sequence of matrix algebras $\left(M_{n}\right)_{n \in \mathbb{N}}$.

Theorem 6.2.5. Let $S$ be a separable operator system, $\mathcal{I}$ an index set and $\left(W_{\alpha}\right), \alpha \in \mathcal{I}$, an increasing sequence of operator systems. Then the following are equivalent.

(i) There is a sequence $S_{\beta} \subset S$ of finite-dimensional sub-operator systems of $S$ such that there union $\bigcup_{\beta} S_{\beta}$ is dense in $S$ and for any triple $(\beta, \varepsilon, n)$ with $\varepsilon>0, n \in \mathbb{N}$, there is an $\alpha \in \mathcal{I}$ and an $(\varepsilon, n)$-order embedding $\phi_{\alpha}: S_{\beta} \rightarrow W_{\alpha}$. 
(ii) There exists a free ultrafilter $\mathcal{U}$ on a subset of $\mathcal{I}$ and a complete order embedding $\phi: S \rightarrow(W)_{\mathcal{U}}$.

Proof. ad (i) $\Rightarrow$ (ii).

We define the following index set,

$$
\mathcal{X}=\left\{\left(S_{\beta}, \varepsilon, n\right) \mid S_{\beta} \subset S \text { finite-dimensional , } \varepsilon>0, n \in \mathbb{N}\right\},
$$

and define the partial ordering

$$
\left(S_{\beta}, \varepsilon_{x}, n_{x}\right) \preccurlyeq\left(S_{\beta^{\prime}}, \varepsilon_{y}, n_{y}\right) \Leftrightarrow S_{\beta} \subset S_{\beta^{\prime}} \& \varepsilon_{x}>\varepsilon_{y} \& n_{x}<n_{y} .
$$

We abbreviate the triple $\left(S_{\beta}, \varepsilon, n\right)$ by $\gamma$. For such a $\gamma$, we introduce the set

$$
\mathcal{X}_{\gamma} \equiv\{\tilde{\gamma} \in \mathcal{X} \mid \gamma \preccurlyeq \tilde{\gamma}\}
$$

and note that the collection $\mathcal{F}$ of these subsets of $\mathcal{X}$ form a filter. Moreover, it follows that

$$
\bigcap_{\gamma \in \mathcal{X}} \mathcal{X}_{\gamma}=\varnothing
$$

Hence the ultrafilter $\mathcal{U}$ dominating the filter $\mathcal{F}$ is free ${ }^{1}$. By assumption, there exists for every $\gamma \in \mathcal{X}$ an $\alpha \in \mathcal{I}$ and a unital linear map $\phi_{\alpha}: S_{\beta} \rightarrow W_{\alpha}$. Hence, for each $\alpha \in \mathcal{I}$, we may define the (non-linear) map $\phi_{\gamma}: \bigcup_{\beta} S_{\beta} \rightarrow W_{\alpha}$,

$$
\phi_{\gamma}(s)=\left\{\begin{array}{l}
\phi_{\alpha}(s) \text { if } s \in S_{\beta} \\
0 \text { else }
\end{array} .\right.
$$

The associated map

$$
\phi: S \rightarrow(W)_{\mathcal{U}}, \quad \phi(s)=q\left(\phi_{\gamma}(s)\right),
$$

where $q: \prod_{\gamma} W_{\alpha \leftrightarrow \gamma} \rightarrow(W)_{\mathcal{U}}$ is the quotient map, extends by density to all $S$. Here, we used the notation $\alpha \leftrightarrow \gamma$ to indicate that the product is taken only over the set of those $W_{\alpha}$ 's which are needed for the approximation. As in the setting of Banach spaces, it follows from the definition of the ultrafilter $\mathcal{U}$ that the mapping $\phi$ is linear, from which it follows by construction that it is unital. We claim that $\phi$ is also completely positive. Suppose not, then there is a positive element $s \in M_{n} \otimes S$ such that $\phi(s) \ngtr 0$. It follows from the Krein-Milman theorem that we can find a completely positive map $\varphi:(W)_{\mathcal{U}} \rightarrow M_{n}$ with $\varphi(\phi(s)) \nsupseteq 0$, or equivalently, there is a unit vector $|\xi\rangle \in \mathbb{C}^{n}$ with $\langle\xi \mid \varphi(\phi(s)) \xi\rangle<0$. Take an arbitrary finite-dimensional subspace $T$ of $S$ with $s \in M_{n} \otimes T$. Since $\phi(T)$ is finite-dimensional and hence reflexive, we can find by Corollary 6.2 .2 a sequence $\left(\varphi_{\gamma}\right)$ of completely positive maps $\varphi_{\gamma}: W_{\gamma} \rightarrow M_{n}$ with

$$
\lim _{\mathcal{U}}\left\langle\xi \mid \varphi_{\gamma}\left(\phi_{\gamma}(s)\right) \xi\right\rangle=\langle\xi \mid \varphi(\phi(s)) \xi\rangle<0 .
$$

Hence there is for $\varepsilon_{0} \ll|\langle\xi \mid \varphi(\phi(s)) \xi\rangle|$ a set $\mathcal{Y} \in \mathcal{U}$ such that for $\gamma \in \mathcal{Y}$ we have $s \in S_{\beta}(\gamma)$, $\varepsilon_{\gamma}<\varepsilon_{0}, n_{\gamma}>n$ and hence there is an $\alpha \in \mathcal{I}$ and a $\left(\varepsilon_{0}, n\right)$-order embedding $\phi_{\alpha}: S_{\beta} \rightarrow W_{\alpha}$, with

$$
\left|\left\langle\xi \mid \varphi_{\alpha}\left(\phi_{\alpha}(s)\right) \xi\right\rangle-\langle\xi \mid \varphi(\phi(s)) \xi\rangle\right| \leq \varepsilon_{0} .
$$

\footnotetext{
${ }^{1}$ Otherwise, the intersection of all of its elements would consists of a singleton, which is impossible since $\mathcal{U}$ dominates $\mathcal{F}$.
} 
Moreover, there is a completely positive map $\psi: S \rightarrow M_{n}$ with $\psi \approx_{\left(\varepsilon_{0}, n\right)} \varphi_{\alpha} \circ \phi_{\alpha}$ which implies that

$$
\left|\left\langle\xi \mid \varphi_{\alpha}\left(\phi_{\alpha}(s)\right) \xi\right\rangle-\langle\xi \mid \psi(s) \xi\rangle\right| \leq \varepsilon_{0} .
$$

Choosing $\varepsilon_{0}$ small enough we find that $\langle\xi \mid \psi(s) \xi\rangle<0$, contradicting the assumption that $s$ was positive. Hence $\phi$ is completely positive. As explained before, in order for $\phi$ to be a complete order embedding, it suffices to construct for every completely positive map $\psi: S \rightarrow M_{n}$ a sequence $\psi_{\alpha}: W_{\alpha} \rightarrow M_{n}$ of completely positive maps such that

$$
\psi(s)=\lim _{\mathcal{U}} \psi_{\gamma}\left(\phi(s)_{\gamma}\right) .
$$

But the existence of such a sequence follows easily from the definition of $(\varepsilon, n)$-order embeddings, as for any $\gamma \in \mathcal{X}$, we can find for $\psi$ a corresponding map $\psi_{\gamma}$ with

$$
\psi \approx_{(\varepsilon, n)} \psi_{\gamma} \circ \phi_{\gamma}
$$

from which we find that for arbitrary $n \in \mathbb{N}$ and $s \in M_{n} \otimes S$

$$
\psi(s)=\lim _{\mathcal{U}} \psi_{\gamma}\left(\phi_{\gamma}(s)\right) .
$$

ad (ii) $\Rightarrow($ i) .

Let $S \subset(W)_{\mathcal{U}}$ be a sub-operator system, and consider elements of the form $x \in M_{n} \otimes S$. If $x \in M_{n} \otimes S$ is positive, and furthermore satisfies that the sum of its diagonal elements is equal to a multiple of the identity, $\operatorname{Tr} x=\sum_{i} x_{i i}=z \mathbb{I}_{S}\left(z \in \mathbb{R}_{+}\right)$, then the span of its matrix elements $\operatorname{span} x \equiv \operatorname{span}_{i j} x_{i j} \subset S$ is clearly an operator subsystem of $S$. Evidently, by by letting go $n$ to infinity, the union of the subspaces $\operatorname{span} x$, with $x$ as before, is dense in $S$. Take an arbitrary sub-operator system span $x$ of such form. We first "scale" $x$ such that $\operatorname{Tr} x=\mathbb{I}_{S}$, and apply Corollary 6.2.4 in order to get a representing sequence $x_{\alpha} \in M_{n} \otimes W_{\alpha}$,

$$
x_{\alpha} \geq 0 \quad \operatorname{Tr} x_{\alpha}=\mathbb{I}_{W_{\alpha}} .
$$

We now define the maps

$$
\phi_{\alpha}: \operatorname{span} x \ni \sum_{i j} a_{i j} x_{i j} \mapsto \sum_{i j} a_{i j} x_{\alpha, i j},
$$

and claim that for any choice $\varepsilon>0$ and $m \in \mathbb{N}$ there is $\alpha_{0} \in \mathcal{I}$ such that $\phi_{\alpha_{0}}$ is an $(\varepsilon, m)$ order embedding. The unitality follows from the rescaling of the element $x$ and Corollary 6.2.4. Let $\varphi: W_{\alpha_{0}} \rightarrow M_{n}$ be a completely positive map. Since we have that for any $\alpha \in \mathcal{I}$ that either $W_{\alpha} \subseteq W_{\alpha_{0}}$ or $W_{\alpha_{0}} \subseteq W_{\alpha}$, we can construct a sequence $\left(\psi_{\alpha}\right)$ of completely positive maps $\psi_{\alpha}: W_{\alpha} \rightarrow M_{n}$ with $\psi_{\alpha_{0}}=\varphi$ by extending or restricting the map $\varphi_{\alpha_{0}}$. By construction, the restriction $\psi \equiv\left(\psi_{\alpha}\right)$ to $\operatorname{span} x$,

$$
\psi\left(\sum_{i j} a_{i j} x_{i j}\right)=\lim _{\mathcal{U}} \sum_{i j} a_{i j} \psi_{\alpha}\left(x_{i j}\right)
$$

is completely positive. We now let $\varepsilon>0$ and $m \in \mathbb{N}$ and consider elements $y \in M_{m} \otimes \operatorname{span} x$ with $\|y\| \leq 1$. For all representing sequences $y_{\alpha} \in M_{m} \otimes W_{\alpha}$, we have $\lim _{\mathcal{U}} \psi_{\alpha}\left(y_{\alpha}\right)=\psi(y)$ and hence there is a set $\mathcal{Y}_{y} \in \mathcal{U}$ such that for $\alpha \in \mathcal{Y}_{y}$ we have

$$
\left\|\psi_{\alpha}\left(\tilde{y}_{\alpha}\right)-\psi(y)\right\|_{M_{n^{2}}} \leq \varepsilon,
$$


for all representing sequences $(\tilde{y})_{\alpha}$ of $y$. By taking a $\delta$-net $\left\{y_{k}\right\}_{1 \leq k \leq p}$ in the compact unit ball of $M_{m} \otimes \operatorname{span} x$ with $\delta$ sufficiently small and setting

$$
\mathcal{X}=\mathcal{Y}_{y_{1}} \cap \mathcal{Y}_{y_{1}} \cap \cdots \cap \mathcal{Y}_{y_{p}}
$$

we find for $\alpha_{0} \in \mathcal{X}$ that

$$
\varphi \circ \phi_{\alpha_{0}} \approx_{(\varepsilon, m)} \psi,
$$

which verifies (ii) in Definition 6.2.2. Now let $\psi: \operatorname{span} x \rightarrow M_{n}$ be a completely positive map. Since $\operatorname{span} x$ is finite-dimensional, it is reflexive, and we can find by Corollary 6.2.2 a sequence of completely positive maps $\psi_{\alpha}: W_{\alpha} \rightarrow M_{n}$ such that for $\sum_{i j} A_{i j} \otimes x_{i j} \in$ $M_{m} \otimes \operatorname{span} x$

$$
\psi\left(\sum_{i j} A_{i j} \otimes x_{i j}\right)=\sum_{i j} A_{i j} \otimes \lim _{\mathcal{U}} \psi_{\alpha}\left(x_{\alpha, i j}\right) .
$$

Using the $\delta$-net $\left\{y_{k}\right\}_{1 \leq k \leq p}$ in the compact unit ball of $M_{m} \otimes \operatorname{span} x$ constructed above and choosing $\alpha_{0}$ as before we find that

$$
\psi_{\alpha_{0}} \circ \phi_{\alpha_{0}} \approx_{(\varepsilon, m)} \psi
$$

which verifies condition (i) in Definition 6.2.2. It follows that $\phi_{\alpha_{0}}$ is an $\left(\varepsilon_{0}, m\right)$-order isomorphism.

In general, and especially in the case of $\mathrm{C}^{*}$-algebras and operators spaces, double duals and ultraproducts are closely related. In the following, we comment on that in the case of operator systems. We first need a little definition and a lemma. We note shortly that if $V$ is another operator system then because of the duality between positive functionals on $M_{n} \otimes V$ and completely positive maps $C P\left(V, M_{n}\right)$ from $V$ to $M_{n}$ every element $v$ of the double dual $V^{* *}$ is a positive map from the space $C P\left(V, M_{n}\right)$ to $M_{n}$.

Lemma 6.2.6. Let $S$ be an operator system, $\mathcal{N}$ a von Neumann algebra and let $\varphi: S \rightarrow \mathcal{N}^{* *}$ be a complete order embedding. Then for every $\varepsilon>0$ and $n \in \mathbb{N}$ there is an $(\varepsilon, n)$-order embedding $\phi: S \rightarrow \mathcal{N}$.

Proof. We first note that since $\mathcal{N}$ is a von Neumann algebra, the dual map $i^{*}: \mathcal{N}^{* *} \rightarrow \mathcal{N}$ of the canonical inclusion map $i: \mathcal{N}_{*} \rightarrow \mathcal{N}^{*}$ is a normal conditional expectation. We claim that the unital completely positive map $T=i^{*} \circ \tilde{\varphi}: S \rightarrow \mathcal{N}$ is an is an $(\varepsilon, n)$-order embedding for any $\varepsilon>0$ and $n \in \mathbb{N}$. According to the discussion after definition 6.2.2, we have to verify the first condition in 6.2.2. Let $\psi: S \rightarrow M_{m}$ be a completely positive and unital map. Since $S \subset \mathcal{N}^{* *}$, there is an extension $\tilde{\psi}: \mathcal{N}^{* *} \rightarrow M_{m}$. The predual of a von Neumann algebra is weak-*-dense in the corresponding dual space, and hence there is for any $\varepsilon>0$ a completely positive map $\psi_{\varepsilon}: \mathcal{N} \rightarrow M_{m}$, corresponding to a state on $M_{n} \otimes \mathcal{N}$ such that

$$
\left\|\tilde{\psi}(x)-x\left(\psi_{\varepsilon}\right)\right\|_{M_{n m}} \leq \varepsilon\|x\|,
$$

for all $x \in M_{n} \otimes \mathcal{N}^{* *}$. Choosing $x=\tilde{\varphi}(s)$, for some $s \in S$ with $\|s\| \leq 1$, we find $x\left(\psi_{\varepsilon}\right)=i\left(\psi_{\varepsilon}\right)(x)=\psi_{\varepsilon}\left(i^{*} \circ \tilde{\varphi}(s)\right)$ and thus

$$
\left\|\psi(s)-\psi_{\varepsilon}(T(s))\right\|_{M_{n m}} \leq \varepsilon .
$$


It remains to turn $\psi_{\varepsilon}$ into a unital map, while remaining its positivity properties. We again apply Paulsen's trick and define for some state $\rho$ on $\mathcal{N}$ the completely positive and unital map

$$
\tilde{\psi}_{\varepsilon}(s)=\frac{1}{\left\|\psi_{\varepsilon}\right\|} \psi_{\varepsilon}(s)+\rho(x)\left(\mathbb{I}_{m}-\frac{1}{\left\|\psi_{\varepsilon}\right\|} \psi_{\varepsilon}\left(\mathbb{I}_{S}\right)\right) .
$$

Since $T$ is unital, and $\left\|\mathbb{1}_{S}\right\|=1$ we have $\left\|\mathbb{I}_{m}-\psi_{\varepsilon}\left(\mathbb{I}_{S}\right)\right\| \leq \varepsilon$ from which it can be seen that the right hand side is bounded by some multiple of $\varepsilon$. It follows that the matrix $\tilde{\psi}_{\varepsilon}(s)$ is close to $\psi(s)$ in norm, and hence the condition is verified.

We obtain the following corollary for the representation of embeddings into double duals of von Neumann algebras. Whether the restriction to von Neumann algebras is needed is not clear to us.

Corollary 6.2.7. Let $S$ be an operator system, $\mathcal{N}$ a von Neumann algebra and $\varphi: S \rightarrow \mathcal{N}^{* *}$ a complete order embedding. Then $S$ can be embedded complete order isomorphically into an ultrapower of $\mathcal{N}$.

\subsection{On approximation properties of operator systems}

Simulation by finite means. We are interested in the following quantum information theoretic question. Suppose we have two parties, as usually named Alice and Bob. Both hold a copy of a quantum system modeled by some operator system $V$. We let both quantum system in question interact with some finite-dimensional one, denoted for simplicity by $M$ (and being modeled by some matrix algebra $M_{n}$ ). This third system can be thought of as some environment, or some third player Alice and Bob cannot influence. Hence the state of the joint system are given by states on $M_{n} \otimes V$, which can be identified with elements of $M_{n} \otimes V^{*}$. Correspondingly, the observables are given by elements of $M_{n} \otimes V$. Suppose now that Alice, after some time, wants to tell Bob which state she has. Can she do so, using a finite amount of (quantum) information, thereby leaving the finite-dimensional system untouched? From physical considerations, this question should be relaxed into whether she can do so approximately. In order to tell Bob, Alice would map her state (restricted to the subsystem $V$ ) into some state on some finite-dimensional quantum system, then send this quantum system to Bob, who would try to invert the map Alice did first and recover the state, then holding a joint state between $V$ and $M$. The figure of merit is whether Bob can, up to some small error, reproduce the expectation values Alice would have measured. In the hole process, the correlations with the third system $M$ are not to be destroyed.

Weak models of operator systems. Formally, Alice action would correspond to some completely positive map $\psi$ from $V^{*}$ into the density operators on some finite-dimensional Hilbert space, say of dimension $m$. Starting with some state $\omega_{M A}$ on $M_{n} \otimes V$, this results in some density operator $\rho_{M T}=\operatorname{id}_{n} \otimes \psi(\omega)$ on $\mathbb{C}^{n} \otimes \mathbb{C}^{m}$. Here, $T$ denotes the quantum system modeled by the Hilbert space $\mathbb{C}^{m}$. Alice would send a finite number of qubits to Bob, thereby transferring her share of the state $\rho_{M T}$ (the part which acts on $T$, for transfer). Bob would then act with some completely positive map $\varphi$, mapping the former part of Alice share of the state $\rho_{M T}$ back into some state $\sigma_{M B}=\mathrm{id}_{n} \otimes \varphi\left(\rho_{M T}\right)$ on the operator system $S$. Hence, the resulting state is given by $\sigma_{M B}=\mathrm{id}_{n} \otimes \varphi \circ \psi\left(\omega_{M A}\right)$. Bob would then 
choose some observable $a \in M_{n} \otimes V$, and the protocol would be successful if we have that for any $\varepsilon>0$,

$$
\left|\left\langle\omega_{M A}, a\right\rangle-\left\langle\operatorname{id}_{n} \otimes \varphi \circ \psi\left(\omega_{M A}\right), a\right\rangle\right| \leq \varepsilon .
$$

Here, $\langle\omega, a\rangle$ just means the expectation value of $a$ in the state $\omega$.

Suppose now that Alice and Bob have some prior knowledge on what they want to achieve. Alice, for example, might have some information about the state she wants to send to Bob, and Bob might have some hint on what observable he would like to measure, and both could "tune" their part of the protocol by using this information. Assuming that the prior information is also finite, this means that they both choose from finite-dimensional subspaces of states respectively observables. Formally, this means that Alice chooses $\omega$ from some subspace $R \subset S^{*}$, and Bob chooses some $a \in S \subset V$. Both the "encoding" map $\psi$ and the "decoding" map $\varphi$ can now depend on the finite dimensional subspace $R$ respectively $S$. Replacing $T$ with some arbitrary operator system $W$ then leads to the following definition.

Definition 6.3.1. Let $V$ be an operator system, and denote by $V$ its dual, considered as an $*$-matrix ordered space. An increasing sequence $\left(W_{\alpha}\right)_{\alpha \in \mathcal{I}}$ of finite-dimensional operator systems is called a weak model for $V$, if there exists for every pair of finite-dimensional subspaces $(R, S), R \subset V^{*}, S \subset V, \mathbb{I}_{V} \in T$ and every $\varepsilon>0$ an $\alpha \in \mathcal{I}$, a pair of completely positive maps $\left(R_{\varepsilon}, S_{\varepsilon}\right), R_{\varepsilon}: R \rightarrow W_{\alpha}^{*}, S_{\varepsilon}: S \rightarrow W_{\alpha}$ with $S_{\varepsilon}$ being unital such that

$$
\left\|\langle b, a\rangle-\left\langle\operatorname{id}_{n} \otimes R_{\varepsilon}[b], \operatorname{id}_{m} \otimes S_{\varepsilon}[a]\right\rangle\right\|_{M_{n m}} \leq \varepsilon
$$

for all elements $b \in M_{n} \otimes R$ and $a \in M_{m} \otimes S$.

We note that in the above inequality, it is enough to consider positive elements $a, b$. Moreover, we can always assume that there exist an element $\delta \in R$ which is a faithful state for the operator system $S$, hence turning also $R$ into an operator system. Thus, the map $R_{\varepsilon}$ can be also considered to be a completely positive unital map in the category of operator systems, since equation (6.5) implies that the faithful state $\delta$ is mapped to some faithful state $\delta_{\alpha}$ on $W_{\alpha}$. We note that the notion of weak models is only interesting for infinite-dimensional operator systems $V$ since otherwise it reduces to the stronger notion of nuclearity.

Theorem 6.3.1. Let $V$ be an infinite-dimensional operator system, and let $\left(W_{n}\right)_{n \in \mathbb{N}}$ be an increasing sequence of finite-dimensional operator systems which is a weak model for $V$. Then there exists an index set $\mathcal{I}$ and a suitable ultrafilter $\mathcal{U}$ on $\mathcal{I}$ such that the following two conditions hold.

(i) The operator system $V$ embeds completely positive into into the ultraproduct operator system $\left(W_{\alpha}\right)_{\mathcal{U}}$.

(ii) The dual space $V^{*}$ embeds as a *-matrix ordered space completely positive into the ultraproduct operator system $\left(W^{*}{ }_{\alpha}\right)_{\mathcal{U}}$.

Moreover, if $R, S$ denote the two order embeddings, then we have that

$$
\langle b, a\rangle=\lim _{\mathcal{U}}\left\langle\operatorname{id}_{n} \otimes R_{\alpha}[b], \operatorname{id}_{m} \otimes S_{\alpha}[a]\right\rangle
$$

for all elements $b \in M_{n} \otimes V^{*}$ and $a \in M_{m} \otimes V$. 
The proof first verifies the existence of embeddings for finite-dimensional subspaces, which basically follows directly from the definition of weak models. The extension to the hole of the space $V$ then follows by a standard ultraproduct technique, see for example [22].

Proof. We focus first on a pair of finite-dimensional subspaces $(R, S) \subset V^{*} \times V$. Let $R_{n}: V^{*} \rightarrow W_{n}^{*}, S_{n}: V \rightarrow W_{n}$ the maps corresponding to $\varepsilon=\frac{1}{n}, n \in \mathbb{N}$. We define the following pair of maps

$$
\begin{array}{ll}
\mathcal{R}: R \rightarrow \prod_{n} W_{n}^{*}, & \mathcal{R}(x)=\left(R_{n}(x)\right)_{n \in \mathbb{N}} \\
\mathcal{S}: S \rightarrow \prod_{n} W_{n}, & \mathcal{S}(x)=\left(S_{n}(x)\right)_{n \in \mathbb{N}}
\end{array}
$$

and since for every $n$ both maps are by definition completely positive, the maps $\mathcal{R}$ and $\mathcal{S}$ are completely positive. In addition, by the same reasoning, $\mathcal{S}$ is unital. Let now $\mathcal{W}$ be a free ultrafilter on the set of natural numbers $\mathbb{N}$, and consider the pair of quotient maps

$$
\begin{aligned}
& q: \prod_{n} W_{n} \rightarrow\left(W_{n}\right)_{\mathcal{W}} \\
& q^{\prime}: \prod_{n} W_{n}^{*} \rightarrow\left(W_{n}^{*}\right)_{\mathcal{W}} .
\end{aligned}
$$

We note that since the operator systems $W_{n}$ are assumed to be of finite dimension, their duals can also be considered as operator systems, and hence the ultraproduct operator system $\left(W^{*}\right)_{\mathcal{W}}$ is well-defined. The compositions $q \circ \mathcal{S}$, and $q^{\prime} \circ \mathcal{R}$ are then also completely positive (and unital for the case of $q \circ \mathcal{S}$ ). Moreover, if $x_{1}, \ldots, x_{m}$ denote a linearly independent set in $S$, then $\mathcal{S}\left(x_{1}\right), \ldots, \mathcal{S}\left(x_{m}\right)$ are representing sequences of $q \circ \mathcal{S}\left(x_{1}\right), \ldots, q \circ \mathcal{S}\left(x_{m}\right)$, fulfilling requirements (i) and (ii) in Proposition 6.2.3. Let now $\psi: V \rightarrow M_{m}$ be a completely positive map, and denote by $\tilde{\psi}$ the corresponding element of $M_{m} \otimes V^{*}$. We may enlarge the space $R \subset V^{*}$ by $\psi$,

$$
\tilde{R}=\operatorname{span}\left(R, \psi_{k l}\right)
$$

where $\psi_{k l}$ are the matrix elements of $\psi, \psi_{k l}(x)=\langle k \mid \psi(x) l\rangle$, which are bounded functionals on $V$. Since $\tilde{R}$ is again a finite-dimensional subspace of $V^{*}$, there also exist a corresponding sequence of maps $R_{n}: \tilde{R} \rightarrow W_{n}^{*}$ and hence a corresponding map $\mathcal{R}: \tilde{R} \rightarrow \prod_{n} W_{n}^{*}$. It follows for this map that

$$
\psi(x)=\langle\tilde{\psi}, x\rangle=\lim _{\mathcal{U}}\left\langle\mathcal{R}[\tilde{\psi}]_{n}, \mathcal{S}[x]_{n}\right\rangle_{n}=\lim _{\mathcal{U}} \mathcal{R}(\psi)_{n}\left[S\left(x_{n}\right)\right] .
$$

Since $\mathcal{R}$ is a completely positive map, $\mathcal{R}[\tilde{\psi}]_{n}$ is a positive element of $M_{m} \otimes W_{n}^{*}$ for every $n$, and can be identified with a completely positive map $\mathcal{R}(\psi)_{n}: W_{n} \rightarrow M_{m}$. Hence, requirement (iii) of Proposition 6.2.3 is also verified, and the map $q \circ \mathcal{S}$ is a completely positive embedding of $V$ into the ultraproduct $\left(W_{n}\right)_{\mathcal{W}}$. The argument can be repeated for the dual space $V^{*}$, replacing the maps $S_{n}$ by $R_{n}$. The requirements of Proposition 6.2.3 can be verified accordingly, and we obtain a completely positive embedding of $V^{*}$ into the ultraproduct $\left(W_{n}^{*}\right)_{\mathcal{W}}$.

Let now $\mathcal{I}$ be the set of all triples of the form $(S, R, p)$, where $S$ is a $p$-dimensional subspace of $V, R$ a $p$-dimensional subspace of $V^{*}$, and $n \in \mathbb{N}$. For convenience, we abbreviate 
$\alpha=(S, R, p)$. Due to the discussion above, for every such $\alpha=(S, R, p)$ there exist a pair of completely positive (and unital in the second case) injective maps

$$
\begin{aligned}
& \mathcal{R}_{\alpha}: R \rightarrow\left(W_{n}^{*}\right)_{\mathcal{W}}, \\
& \mathcal{S}_{\alpha}: S \rightarrow\left(W_{n}\right)_{\mathcal{W}} .
\end{aligned}
$$

For a pair of elements $a \in V, b \in V^{*}$ and $m \in \mathbb{N}$ we define

$$
I_{a, b, m} \equiv\{\alpha \in \mathcal{I} \mid a \in S, b \in R, m \geq p\}
$$

and since for finite sets $a_{1}, \ldots, a_{k} \in V, b_{1}, \ldots, b_{k} \in V^{*}, m_{1}, \ldots, m_{k} k \in \mathbb{N}$ the intersections

$$
I_{a_{1}, b_{1}, m_{1}} \cap \cdots \cap I_{a_{k}, b_{k}, m_{k}}
$$

are non-empty, there exists an ultrafilter $\mathcal{V}$ on the index set $\mathcal{I}$ such that

$$
\left\{I_{a, b, m} \mid a \in V, b \in V^{*}, m \in \mathbb{N}\right\} \subset \mathcal{V} .
$$

We then define maps and extend them non-linearly

$$
\begin{aligned}
& \mathcal{R}: V^{*} \rightarrow \prod_{\alpha}\left(W_{n}^{*}\right)_{\mathcal{W}}, \mathcal{R}=\prod_{\alpha}(\mathcal{R}(a))_{\alpha}, \quad(\mathcal{R}(a))_{\alpha}=\left\{\begin{array}{l}
\mathcal{R}_{\alpha}(a) \text { if } a \in R \\
0 \text { else }
\end{array}\right. \\
& \mathcal{S}: V \rightarrow \prod_{\alpha}\left(W_{n}\right)_{\mathcal{W}}, \mathcal{S}=\prod_{\alpha}(\mathcal{S}(a))_{\alpha}, \quad(\mathcal{S}(b))_{\alpha}=\left\{\begin{array}{l}
\mathcal{S}_{\alpha}(b) \text { if } b \in S \\
0 \text { else }
\end{array}\right.
\end{aligned}
$$

Let $q$ and $q^{\prime}$ be the quotient maps corresponding to the ultrafilter $\mathcal{V}$ and letting $\mathcal{U}=\mathcal{V} \times \mathcal{W}$ be the product ultrafilter, we have

$$
\begin{aligned}
& q: \prod_{\alpha} W_{\alpha} \rightarrow\left(\left(W_{n}\right)_{\mathcal{W}}\right)_{\mathcal{V}}=\left(W_{\alpha}\right)_{\mathcal{U}} \\
& q^{\prime}: \prod_{\alpha} W_{\alpha}^{*} \rightarrow\left(\left(W_{n}^{*}\right)_{\mathcal{W}}\right)_{\mathcal{V}}=\left(W_{\alpha}^{*}\right)_{\mathcal{U}} .
\end{aligned}
$$

Then, due to the definition of the index set $\mathcal{I}$ and the ultrafilter $\mathcal{U}$, the compositions $q^{\prime} \circ \mathcal{R}$ and $q \circ \mathcal{S}$ are linear and completely positive. Moreover, their inverses are completely positive, and hence order isomorphisms in the category of matrix $*$-ordered spaces. It is now easy to check that $q \circ \mathcal{S}$ is also unital, and thus also an order isomorphism in the category of operator systems. Moreover, the equality

$$
\langle a, b\rangle=\lim _{\mathcal{U}}\left\langle\operatorname{id}_{n} \otimes R_{\alpha}[b], \operatorname{id}_{m} \otimes S_{\alpha}[a]\right\rangle
$$

also follows from the construction.

As outlined in the introduction of this section, the definition of weak models sounds very tempting from the physical point of view. It basically asks for approximations of a finite collection of states evaluated on a finite number of observables, if we model the linear span of the later set as an operator system sitting in the operator system of all observables. We would normally choose this "big" operator system as some $\mathrm{C}^{*}$-algebra, which models the structure of observables of some physical system. A particular example would be the 
observable algebra $\mathcal{A}$ of an (finite or infinite) spin system, where all local constituents are modeled by a finite-dimensional matrix algebra. Since then the $\mathrm{C}^{*}$-algebra $\mathcal{A}$ is a UHF algebra, it is in particular a nuclear $\mathrm{C}^{*}$-algebra. As explained in the chapter 2 , these algebras can be factorized approximately through matrix-algebras, and it is easy to see that this implies that the set of finite-dimensional matrix algebras is a weak model for $\mathcal{A}$. Since the requirement of weak models is expressed through the evaluation of a finite number of states, the same reasoning can be applied to hyperfinite von Neumann algebras. Applying the preceding theorem 6.3.1 to these examples, we obtain an embedding of the algebra into an ultraproduct of matrix algebras, together with a corresponding embedding of the dual space into the ultraproduct of the corresponding dual spaces of matrix algebras, which are completely order isomorphic to matrix algebras. Hence the implications of theorem 6.3.1 are much weaker than the assumption of nuclearity we started with. Thus, the class of $\mathrm{C}^{*}$-algebras which have a weak model of matrix algebras is bigger than nuclear $\mathrm{C}^{*}$ algebras. Having the last chapter in mind, one might be tempted to conjecture that all QWEP algebras have the matrix algebras as a weak model. We were not able to either prove or refute this conjecture. On the contrary, we can prove that the existence of such weak models imply QWEP.

Ultraproducts of $\mathcal{B}(\mathcal{H})$. In order to simplify the analysis, we note that we can, for all $n \in \mathbb{N}$, embed $M_{n}$ in a completely positive way into the operator system $\mathcal{B}(\mathcal{H})$, where $\mathcal{H}$ is for example the separable Hilbert space $\ell_{2}(\mathbb{Z})$. It follows that we have to study the properties of the $\mathrm{C}^{*}$-algebra

$$
(\mathcal{B}(\mathcal{H}))_{\mathcal{U}}
$$

Fortunately, this has been already done, see for example the first part of the paper by Raynaud, [77]. Its weak closure in $\mathcal{B}\left((\mathcal{H})_{\mathcal{U}}\right)$, where the inclusion is the canonical diagonal map, mapping a sequence of operators, each acting on $\mathcal{H}$, onto an operator acting on a sequence of elements in $\mathcal{H}$, is a von Neumann algebra with predual being the ultraproduct of trace-class operators $\left(S_{1}(\mathcal{H})\right)_{\mathcal{U}}$. The fact that $\left(S_{1}(\mathcal{H})\right)_{\mathcal{U}}^{*}$ is indeed a von Neumann algebra can be seen from the following reasoning. The ultraproduct

$$
\left(S_{1}(\mathcal{H})\right)_{\mathcal{U}} \subset\left(\prod_{\alpha \in \mathcal{I}} \mathcal{B}(\mathcal{H})\right)^{*}
$$

is invariant under the conjugation by left or right elements of $\prod_{\alpha} \mathcal{B}(\mathcal{H})$ and hence there exists, according to [83], Theorem III.2.7 a central projection $\mathcal{P}_{\mathcal{U}}$ in the enveloping von Neumann algebra $\left(\prod_{\alpha \in \mathcal{I}} \mathcal{B}(\mathcal{H})\right)^{* *}$ such that

$$
\left(S_{1}(\mathcal{H})\right)_{\mathcal{U}}^{*}=\mathcal{P}_{\mathcal{U}}\left(\prod_{\alpha \in \mathcal{I}} \mathcal{B}(\mathcal{H})\right)^{*}
$$

Hence we may identify $\left(S_{1}(\mathcal{H})\right)_{\mathcal{U}}^{*}$ with the von Neumann algebra $\mathcal{P}_{\mathcal{U}}\left(\prod_{\alpha \in \mathcal{I}} \mathcal{B}(\mathcal{H})\right)^{* *}$. Moreover, we have that

$$
\mathcal{P}_{\mathcal{U}}\left((\mathcal{B}(\mathcal{H}))_{\mathcal{U}}^{* *}\right)_{*}=\left(S_{1}(\mathcal{H})\right)_{\mathcal{U}}
$$

For details see [32]. We note that the constructed von Neumann algebra $\mathcal{N}_{\mathcal{U}}(\mathcal{H}) \equiv\left(S_{1}(\mathcal{H})\right)_{\mathcal{U}}^{*}$ has the QWEP property. Indeed, since $\mathcal{B}(\mathcal{H})$ is injective and hence in particular has the WEP, the $\mathrm{C}^{*}$-algebra $\prod_{\alpha \in \mathcal{I}} \mathcal{B}(\mathcal{H})$ and its double dual are QWEP, see Proposition 4.1 in 
[69]. But $\mathcal{N}_{\mathcal{U}}(\mathcal{H})$ is a quotient of $\left(\prod_{\alpha \in \mathcal{I}} \mathcal{B}(\mathcal{H})\right)^{* *}$, and hence also is QWEP. In fact, $\mathcal{N}_{\mathcal{U}}(\mathcal{H})$ can be understood as the "universal" QWEP von Neumann algebra, in view of the next results by Junge.

Theorem 6.3.2 (Junge, Lemmas 2.1 and 2.13 in [44]). Let $\mathcal{N}$ be a von Neumann algebra which is $Q W E P, \mathcal{H}$ be a Hilbert space and $S_{1}(\mathcal{H})$ the Banach space of trace-class operators. Then there exist an ultrafilter $\mathcal{U}$ on the set of natural numbers $\mathbb{N}$, a completely positive embedding $E: \mathcal{N}^{*} \rightarrow\left(S_{1}(\mathcal{H})\right)_{\mathcal{U}}$, a normal $*$-homomorphism $\pi: \mathcal{N}^{* *} \rightarrow\left(S_{1}(\mathcal{H})\right)_{\mathcal{U}}^{*}$ such that

$$
\psi(x)=\operatorname{Tr}_{\mathcal{U}} E(\psi) \pi(x)
$$

where $\operatorname{Tr}_{\mathcal{U}}$ is the ultraproduct trace.

Using that the Banach space of trace-class operators possesses "nice" finite-dimensional approximations, basically being the restriction to finite-dimensional subspaces of $\mathcal{H}$, Raynaud obtained the following.

Lemma 6.3.3 (Raynaud, Lemmas 1.15 and 1.16 in [77]). Let $\mathcal{U}$ be an ultrafilter on some index set, and let $S_{1}^{m}$ be the Banach space of trace-class operators on the Hilbert space $\mathbb{C}^{m}$, $m \in \mathbb{N}$. Then there exist a suitable ultrafilter $\mathcal{V}$ on the set of natural numbers $\mathbb{N}$ such that the ultrapower $\left(S_{1}(\mathcal{H})\right)_{\mathcal{U}}^{*}$ is $C^{*}$-isomorphic to a $C^{*}$-subalgebra of the ultraproduct $\left(S_{1}^{m}\right)_{\mathcal{V}}^{*}$ which is the range of a normal conditional expectation.

Hence, if a von Neumann algebra is QWEP, we may identify its predual completely positive with a subspace in the ultraproduct $\left(S_{1}^{m}\right)_{\mathcal{V}}$. Since the Banach space $S_{1}^{m}$ is completely order isomorphic to $M_{m}^{*}$, the identity being the trace, the ultraproduct $\left(S_{1}^{m}\right)_{\mathcal{V}}$ is an ultraproduct of the dual operator systems $M_{m}^{*}$. But the operator system $M_{m}$ is completely order isomorphic to its dual $M_{m}^{*}$. This order isomorphism does not only hold in the category of matrix *-ordered spaces, but is also a complete order isomorphism in the category of operator systems, if the identity matrix $\mathbb{I}_{m}$ is identified with the trace functional on $M_{m}$. This shows that the ultraproduct operator system $\left(S_{1}^{m}\right)_{\mathcal{V}}$ can be identified with the ultraproduct operator system $\left(M_{m}\right)_{\mathcal{V}}$, the identity being the sequence of trace functionals.

Implications of weak models. If now some operator system $V$ has $\left(M_{n}\right)_{n \in \mathbb{N}}$ as a weak model, then we obtain a complete order embedding $\phi$ of $V$ into the ultraproduct $(\mathcal{B}(\mathcal{H}))_{\mathcal{U}}$, which in turn embeds into the QWEP algebra $\mathcal{N}_{\mathcal{U}}(\mathcal{H})$. We now analyze its dual. Since the dual operator systems $M_{n}^{*}$ can be embedded into the trace-class operators $S_{1}(\mathcal{H})$ on $\mathcal{H}^{2}$, we obtain a completely positive embedding $\varphi$ of the dual space $V^{*}$ into the ultraproduct $\left(S_{1}(\mathcal{H})\right)_{\mathcal{U}}{ }^{3}$. Conversely, each element $\widehat{\left(t_{\alpha}\right)} \in\left(S_{1}(\mathcal{H})\right)_{\mathcal{U}}$ defines a bounded functional on $V$ by

$$
\left\langle\widehat{\left(t_{\alpha}\right)}, v\right\rangle \equiv \lim _{\mathcal{U}}\left\langle\phi(v)_{\alpha}, t_{\alpha}\right\rangle
$$

where $\left(t_{\alpha}\right)$ and $\phi(v)_{\alpha}$ are any representing sequences of $\widehat{\left(t_{\alpha}\right)}$ resp. $\phi(v) \in(\mathcal{B}(\mathcal{H}))_{\mathcal{U}} \subset$ $\mathcal{N}_{\mathcal{U}}(\mathcal{H})$. The associated map $\theta$ is easily seen to be completely positive. Moreover, we

\footnotetext{
${ }^{2}$ This can for example be achieved by adding sufficiently many zeros. The obtained embedding is clearly completely positive.

${ }^{3}$ We note however, that this map is understood as a morphism in the category of matrix-*-ordered vector spaces.
} 
have due to equation (6.6) that the composition satisfies $\theta \circ \varphi=\mathrm{id}_{V^{*}}$, and by duality $\varphi^{*} \circ \theta^{*}=\mathrm{id}_{V^{* *}}$. Since $\phi$ is unital, the dual map $\theta^{*}$ is also unital (and completely positive). It follows that

$$
\varphi^{*}\left(\mathbb{I}_{\mathcal{N}_{\mathcal{U}}(\mathcal{H})}\right)=\varphi^{*} \theta^{*}\left(\mathbb{I}_{V^{* *}}\right)=\varphi^{*} \theta^{*}\left(\mathbb{I}_{V}\right)=\mathbb{I}_{V}
$$

since the operator system is completely order embedded in its double dual $V^{* *}$, see e.g. Theorem 1.2 in [50]. By composition, we obtain a completely positive and unital projection $E=\theta^{*} \circ \varphi^{*}$ onto the operator system $\theta^{*}\left(V^{* *}\right)$. Moreover, $\varphi^{*} \circ E \circ \theta^{*}=\mathrm{id}_{V^{* *}}$. If now the operator system $V$ is completely positive isomorphic to a $\mathrm{C}^{*}$-algebra $\mathcal{A}$, then we obtain a normal conditional expectation from the QWEP algebra $\mathcal{N}_{\mathcal{U}}(\mathcal{H})$ onto $\mathcal{A}^{* *}$. By condition (ii) in Proposition 4.1 in [69], it follows that $\mathcal{A}$ is QWEP.

We note that the assumption that the operator system $V$ is a $\mathrm{C}^{*}$-algebra is not necessary. Indeed, the normal conditional expectation $E$ from the QWEP $\mathrm{C}^{*}$-algebra $\mathcal{N}_{\mathcal{U}}(\mathcal{H})$ onto the double dual $V^{* *}$ can be used to define a product on $V^{* *}$, setting for $a, b \in V^{* *}$

$$
a \circ b \equiv E\left(\theta^{*}(a) \cdot \theta^{*}(b)\right)
$$

where the product is taken in the $\mathrm{C}^{*}$-algebra $\mathcal{N}_{\mathcal{U}}(\mathcal{H})$. This induced product was first defined by Choi and Effros [15] in the study of injective operator systems, with the $\mathrm{C}^{*}$ algebra $\mathcal{N}_{\mathcal{U}}(\mathcal{H})$ replaced by $\mathcal{B}(\mathcal{H}){ }^{4}$. Using the complete positivity of the map $E$ together with the fact that it agrees with the identity on $V^{* *}$ (or on $V$, in the case of injective operator systems), it can be sown that this product gives rise to a $\mathrm{C}^{*}$-structure on $V^{* *}$ (resp. $V$ ). For details, we refer the reader to chapter 15 of the book by Paulsen, [70]. We note that the analysis for the case studied here is completely analogous to the study of injective operator systems, except that the resulting $\mathrm{C}^{*}$-algebra is of course not longer injective. However, due to the discussion above, it has the QWEP property. We summarize our argument which was along the lines of Lemma 2.13 in the paper [44] by Junge.

Corollary 6.3.4. Let $V$ be an operator system such that the sequence $\left(M_{n}\right)_{n \in \mathbb{N}}$ is a weak model for $V$. Then its double dual $V^{* *}$ is completely order isomorphic to a $C^{*}$-algebra which is a quotient of a $C^{*}$-algebra with the weak expectation property.

Applying this result to a finite-dimensional operator system $S$, we obtain that $S \simeq S^{* *}$ is completely order isomorphic to a $\mathrm{C}^{*}$-algebra. It is then trivial that $S$ is QWEP, since any finite-dimensional $\mathrm{C}^{*}$-algebra is a direct sum of matrix algebras and in particular is nuclear. It follows that in understanding the structure of finite-dimensional operator systems, the requirements of a weak model are too restrictive.

Weak local models and simulation of quantum channels. As outlined in the last section, the requirements on an operator system (or a sequence of operator systems) to be a weak model for another operator system are too strong to infer that all QWEP algebras have this property. Hence it is naturally to ask for weaker conditions, which somehow capture the physical intuition behind the definition of weak models. This section is concerned with such a weakening, and we show that it is indeed in a certain way equivalent to the QWEP condition. Not surprisingly, at least if we have the results on Tsirelson's problem in mind,

\footnotetext{
${ }^{4}$ If $V$ is an injective operator system, the inclusion $i: V \rightarrow i(V) \subset \mathcal{B}(\mathcal{H})$ for some Hilbert space $\mathcal{H}$ extends to a completely positive map $\varphi: \mathcal{B}(\mathcal{H}) \rightarrow V$, being equal to the identity on $i(V) \simeq V$. This map gives the desired conditional expectation on $V$.
} 
is the strong connection to tensor products and hence joint systems. We recall the fact that if $S$ is an operator system, and $\mathcal{A}$ a $\mathrm{C}^{*}$-algebra, then the commuting tensor product equals the maximal tensor product in the category of operator systems, $S \otimes_{c} \mathcal{A} \cong S \otimes_{\max } \mathcal{A}$. The dual space of $S \otimes_{\max } \mathcal{A}$ can be identified with the space of bilinear jointly positive maps $\psi: S \times \mathcal{A} \rightarrow M_{n}$ by the usual linearization procedure,

$$
\psi\left(\sum_{i} s_{i} \otimes a_{i}\right) \equiv \sum_{i} \psi\left(s_{i}, a_{i}\right)
$$

Recall that a bilinear map $\psi: S \times \mathcal{A} \rightarrow M_{n}$ is jointly completely positive if for any pair of positive elements $x \in M_{n} \otimes S, a \in M_{m} \otimes \mathcal{A}$ we have

$$
\psi(x, a) \in M_{n m,+} .
$$

Every bilinear jointly completely positive map $\psi$ on $S \times \mathcal{A}$ with values in the complex numbers defines two completely positive maps $\hat{\psi}_{L}: S \rightarrow \mathcal{A}^{*}$ and $\hat{\psi}_{R}: \mathcal{A} \rightarrow S^{*}$ via the identifications

$$
\hat{\psi}_{L}(s)(a)=\psi(s \otimes a)=\hat{\psi}_{R}(a)(s) .
$$

The converse statement gives that any completely positive map $\varphi: S \rightarrow \mathcal{A}^{*}$ defines a positive (and hence completely positive) functional $\tilde{\varphi}$ on $S \otimes_{\max } \mathcal{A}$ via the identification $\tilde{\varphi}(s \otimes a)=\varphi(s)(a)$. We refer the reader to [71] for a more detailed discussion of jointly completely positive maps.

In view of corollary 5.2.2, we are interested in the range of completely positive maps on the joint system $S \otimes_{\max } \mathcal{A}$. In view of the results of chapter 5 , we primarily consider the case where $\mathcal{A}=\phi(S)^{\prime}$, where $\phi: S \rightarrow \mathcal{B}(\mathcal{H})$ is any complete order embedding.

Definition 6.3.2. Let $S$ be an operator system. We say that $S$ admits a weak local model, if there exists a Hilbert space $\mathcal{H}$ such that for every $\varepsilon>0$, every finite-dimensional sub-operator systems $V \subset S, \mathcal{T} \subset \mathcal{A}$ of some $\mathrm{C}^{*}$-algebra $\mathcal{A}$

(i) there exists a completely positive unital map $\phi_{\varepsilon}: V \rightarrow \mathcal{B}(\mathcal{H})$, and

(ii) for every completely positive map unital $\psi: \mathcal{A} \rightarrow S^{*}$ there exists a completely positive unital map $\psi_{\varepsilon}: \mathcal{T} \rightarrow \mathcal{B}(\mathcal{H})^{*}$

such that for every $t \in \mathcal{T}$ and $v \in V$

$$
\left\|\psi(t)(v)-\psi_{\varepsilon}(t)\left(\phi_{\varepsilon}(v)\right)\right\| \leq \varepsilon\|t\|\|s\| .
$$

We note that if an operator system $S$ admits $\mathcal{B}(\mathcal{H})$ as weak model, then it has a weak local model. Indeed, there exists by assumption a completely positive map of $S$ into $\mathcal{B}(\mathcal{H})$, and the range of $\psi$ restricted to some finite-dimensional subspace of $\mathcal{A}$ is a finite-dimensional subspace of $S^{*}$, for which by assumption there exists a completely positive $R$ map into $\mathcal{B}(\mathcal{H})^{*}$ with the required approximation property. Hence $\left.R \circ \psi\right|_{\mathcal{T} \subset \mathcal{A}}$ fulfills requirement (ii) of the foregoing definition, and (i) follows as explained from the definition of weak models. We note that the requirements for weak local models are less restrictive than for weak models, since the dependence of $\psi_{\varepsilon}$ on $\psi$ does not have to be linear. However, there are sufficient to ensure that $S$ is QWEPCP.

Proposition 6.3.5. Let $S$ be an operator system admitting a weak local model. It follows that $S$ is completely positive complemented by a QWEP von Neumann algebra. 
Remark 6.3.6. We note that for the implication to hold, the requirements of definition 6.3.2 only have to be verified for a collection of finite-dimensional subspaces of $S$ and $\mathcal{A}$ such that their union is dense in $S$, respectively $\mathcal{A}$.

Proof. We let $\mathcal{T}_{\alpha}$ be a collection of finite-dimensional subspaces of $\mathcal{A}$ such that their union is dense in $\mathcal{A}$. We let $\alpha \in \mathcal{I}_{\mathcal{A}}$ be the set of pairs $\left(\mathcal{T}_{\alpha}, \varepsilon_{\alpha}\right)$, where $\varepsilon_{\alpha}>0$ and define as usual a partial order on $\mathcal{I}_{\mathcal{A}}$ by setting

$$
\left(\mathcal{T}_{\alpha}, \varepsilon_{\alpha}\right) \preccurlyeq\left(\mathcal{T}_{\alpha^{\prime}}, \varepsilon_{\alpha^{\prime}}\right) \Leftrightarrow \mathcal{T}_{\alpha} \subset \mathcal{T}_{\alpha^{\prime}} \& \varepsilon_{\alpha}>\varepsilon_{\alpha^{\prime}} .
$$

We define the index set $\mathcal{I}_{S}$ accordingly, with respect to some collection $\left(V_{\kappa}\right)$ of finitedimensional subspaces of $S$ (again assuming that their union is dense), and equip $\mathcal{I}_{S}$ with the same partial ordering. We abbreviate the elements of $\mathcal{I}_{\mathcal{A}}$ by $\alpha$ and the elements of $\mathcal{I}_{S}$ by $\kappa$. As in the proof of theorem 6.2.5, we let $\mathcal{V}$ and $\mathcal{W}$ be the free ultrafilters dominating the filters given by the collection of sets

$$
\mathcal{I}_{\alpha} \equiv\left\{\tilde{\alpha} \in \mathcal{I}_{\mathcal{A}} \mid \alpha \preccurlyeq \tilde{\alpha}\right\}, \quad \mathcal{I}_{\kappa} \equiv\left\{\tilde{\kappa} \in \mathcal{I}_{S} \mid \kappa \preccurlyeq \tilde{\kappa}\right\} .
$$

By assumption, for every $\kappa$ there exists a completely positive unital map $\phi_{\kappa}: V_{\kappa} \rightarrow \mathcal{B}(\mathcal{H})$. Likewise, for every $\alpha \in \mathcal{I}_{\mathcal{A}}$ there exists a completely positive embedding $\iota_{\alpha}: \mathcal{T}_{\alpha} \rightarrow \mathcal{A}$, where $\iota_{\alpha}$ denotes the canonical inclusion map. Hence, we may define maps on $S$ and $\mathcal{A}$ by setting

$$
\begin{gathered}
\phi_{\kappa}(s)=\left\{\begin{array}{l}
\phi_{\kappa}(s) \in \mathcal{B}(\mathcal{H}) \text { if } s \in V_{\kappa}, \\
0 \text { else }
\end{array}\right. \\
\phi_{\alpha}(a)=\left\{\begin{array}{l}
\iota_{\alpha}(a) \in \mathcal{A} \text { if } a \in \mathcal{T}_{\alpha} \\
0 \text { else }
\end{array}\right.
\end{gathered}
$$

It follows that for some fixed pair $\alpha \in \mathcal{I}_{\mathcal{A}}, \kappa \in \mathcal{I}_{S}$, by the functorial property of the commuting tensor product,

$$
\phi_{\kappa} \otimes \iota_{\alpha}: V_{\kappa} \otimes_{c} \mathcal{T}_{\alpha} \rightarrow \mathcal{B}(\mathcal{H}) \otimes_{\max } \mathcal{T}_{\alpha}
$$

is a linear and completely positive and unital map, where we used that since $\mathcal{B}(\mathcal{H})$ is a $\mathrm{C}^{*}$-algebra, $\mathcal{B}(\mathcal{H}) \otimes_{c} \mathcal{T}_{\alpha}=\mathcal{B}(\mathcal{H}) \otimes_{\max } \mathcal{T}$. Furthermore, if we let $q_{S}$ be the quotient map from $\prod \mathcal{B}(\mathcal{H})$ to $(\mathcal{B}(\mathcal{H}))_{\mathcal{W}}$ and $q_{\mathcal{A}}$ the canonical quotient map from $\prod_{\alpha} \mathcal{T}_{\alpha}$ to $\left(\mathcal{T}_{\alpha}\right)_{\mathcal{V}}$, then

$$
\varphi \equiv q_{S} \circ\left(\phi_{\kappa}\right) \otimes q_{\mathcal{A}} \circ\left(\iota_{\alpha}\right): S \otimes_{\max } \mathcal{A} \rightarrow(\mathcal{B}(\mathcal{H}))_{\mathcal{W}} \otimes_{\max }\left(\mathcal{T}_{\alpha}\right)_{\mathcal{V}}
$$

is completely positive and unital, since the maximal tensor product is projective. Any completely positive map $\tilde{\psi}: S \otimes_{\max } \mathcal{A} \rightarrow M_{n}$, for some $n \in \mathbb{N}$, can be identified with a completely positive map $\psi: M_{n}(\mathcal{A}) \rightarrow S^{*}$, and since the assumptions hold for any $\mathrm{C}^{*}$ algebra $\mathcal{A}$, we may replace $\mathcal{A}$ with $M_{n}(\mathcal{A})$ and obtain for every pair $(\alpha, \kappa)$ a completely positive map $\chi_{(\alpha, \kappa)}: \mathcal{T}_{\alpha} \rightarrow \mathcal{B}(\mathcal{H})^{*}$ such that

$$
\psi(a)(s) \approx_{\max \left(\varepsilon_{\alpha}, \varepsilon_{\kappa}\right)} \chi_{(\alpha, \kappa)}\left(\iota_{\alpha}(a)\right)\left(\phi_{\kappa}(s)\right),
$$

for $a \in \mathcal{T}_{\alpha}$ and $s \in V_{\kappa}$. Hence, if we let $\mathcal{U}=\mathcal{V} \times \mathcal{W}$ be the product ultrafilter, we obtain a sequence of completely positive maps $\left(\chi_{(\alpha, \kappa)}\right)$ converging along $\mathcal{U}$ to $\psi$,

$$
\psi(a)(s)=\lim _{\mathcal{U}} \chi_{(\alpha, \kappa)}\left(\left(a_{\alpha}\right),\left(s_{\kappa}\right)\right),
$$


where $\left(a_{\alpha}\right)$ (resp. $\left.\left(s_{\kappa}\right)\right)$ are any representing sequences of $a \in \mathcal{A}$ (resp. $\left.s \in(\mathcal{B}(\mathcal{H}))_{\mathcal{W}}\right)$. It follows that $\varphi$ is a complete order embedding. We now show that (iv) of theorem 5.1.2 holds. Let $\phi: S \rightarrow \mathcal{B}(\mathcal{K})$ be some complete order embedding. We consider the completely positive map $\phi: S \otimes_{\max } \phi(S)^{\prime} \rightarrow \mathcal{B}(\mathcal{K})$ given by $\phi(s \otimes x)=s x$. We apply the foregoing discussion to $\mathcal{A}=\phi(S)^{\prime}$. Since $\mathcal{B}(\mathcal{K})$ is injective we obtain the following commuting diagram,

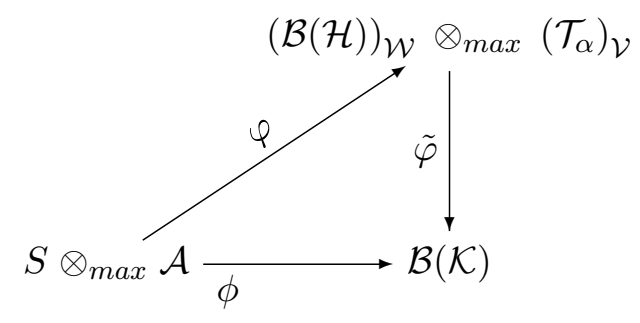

and $\tilde{\varphi}:(\mathcal{B}(\mathcal{H}))_{\mathcal{W}} \otimes_{\max }\left(\mathcal{T}_{\alpha}\right)_{\mathcal{V}} \rightarrow \mathcal{B}(\mathcal{K})$ is a completely positive extension of $\phi$. By the fact that $\tilde{\varphi}$ extends $\phi$, it then follows that $\tilde{\varphi}\left(\left(\mathbb{I}_{\mathcal{K}} \otimes(x)_{\mathcal{V}}\right)=x\right.$, for any representing sequence $(x)_{\mathcal{V}}$ of $x \in \phi(S)^{\prime}$. and hence by Choi's theorem on multiplicative domains, see [70], $\tilde{\varphi}$ is a $\phi(S)^{\prime}$-bimodule map. We define $E:\left(\mathcal{B}(\mathcal{H}) \otimes_{\max } \mathbb{I}_{\mathcal{K}}\right)_{\mathcal{U}} \rightarrow \mathcal{B}(\mathcal{K})$ as $E\left((a)_{\mathcal{U}}\right)=\tilde{\varphi}\left((a)_{\mathcal{U}} \otimes \mathbb{I}_{\mathcal{K}}\right)$. By the bimodule property, we have

$$
E(a) x=\tilde{\varphi}\left((a)_{\mathcal{W}} \otimes \mathbb{I}_{\mathcal{H}}\right) x=\tilde{\varphi}\left((a \otimes x)_{\mathcal{U}}\right)=x \tilde{\varphi}\left((a)_{\mathcal{W}} \otimes \mathbb{I}_{\mathcal{K}}\right)=x E(a)
$$

and hence $E(a) \in \phi(S)^{\prime \prime}$ for every $a \in\left(\mathcal{B}(\mathcal{H}) \otimes_{\max } \mathbb{I}_{\mathcal{K}}\right)_{\mathcal{U}}$. Moreover, $E(s)=s$ for every $s \in S$. We finally note that as $\mathcal{B}(\mathcal{H})$ is injective, $\prod_{\alpha} \mathcal{B}(\mathcal{H}) \simeq \prod_{\alpha} \mathcal{B}(\mathcal{H}) \otimes \mathbb{I}_{\mathcal{K}}$ and also its quotient $\left(\mathcal{B}(\mathcal{H}) \otimes_{\max } \mathbb{I}_{\mathcal{K}}\right)_{\mathcal{U}}$ is QWEP. This proves that $S$ is QWEPCP.

We end this chapter by an exemplary statement which, if true, would imply QWEPCP of some operator system in question. It can be seen as another quantum information theoretic reformulation of approximation properties of operator systems.

Example 6.3.1. Suppose Alice and Bob share a quantum system which on Alice's side is modeled by some operator system $S$, and on Bob's side by some $\mathrm{C}^{*}$-algebra $\mathcal{A}$. We may for example think of $\mathcal{A}$ as the commutant $S^{\prime}$ computed with respect to some complete order embedding $S \subset \mathcal{B}(\mathcal{H})$. They both have access to some joint state $\omega: S \otimes_{\max } \mathcal{A} \rightarrow \mathbb{C}$, i.e. a completely positive map satisfying $\omega\left(\mathbb{I}_{S} \otimes \mathbb{I}_{\mathcal{A}}\right)=1$. Using this system, they want to transfer the information stored in finite-dimensional quantum systems (say $M_{n}$ ) from Alice to Bob. They do this by acting with completely positive maps $S: M_{n} \rightarrow S$ and $A: M_{n} \rightarrow \mathcal{A}$ on their subsystems, followed by the action of the state $\omega$ and then using the so-defined state on $M_{n} \otimes M_{n}$ in order to run usual quantum information theoretic protocols. If we want to study the power these "meta-protocol", we have to study positive matrices of the form

$$
\omega(s \otimes a) \in M_{n^{2}}
$$

with $s \in\left(M_{n} \otimes S\right)_{+}$and $a \in\left(M_{n} \otimes \mathcal{A}\right)_{+}$, as given by the identification of completely positive maps from $M_{n}$ to $S$ (resp. $M_{n}$ to $\mathcal{A}$ ) with positive elements $s \in\left(M_{n} \otimes S\right)_{+}$(resp. $\left.a \in\left(M_{n} \otimes \mathcal{A}\right)_{+}\right)$. If we require these maps to be unital, then we have that the sum of the diagonal elements of $s$ and $a$ equals the respective unit element. We are let to consider the subspaces span $s \subset S$ and span $a \subset \mathcal{A}$. By assumption, we have that $\mathbb{1}_{S} \in \operatorname{span} s$ and $\mathbb{I}_{\mathcal{A}} \in \operatorname{span} a$, which implies that both subspaces are operator systems. Since $\omega(s \otimes a) \in M_{n^{2}}$ 
is a positive matrix, it can be identified with a completely positive map $T$ from $M_{n}$ into $M_{n}$. Assume now that for all $\varepsilon>0$ there exists $k \in \mathbb{N}$ such that

$$
d_{c b}\left(\bigotimes_{i=1}^{k} T, R_{k}\right) \leq \varepsilon,
$$

where $R_{k}$ is a completely positive map corresponding to a positive matrix of the form

$$
\sigma_{k}\left(\phi_{k}\left(\mathbb{1}_{n^{k-1}} \otimes s\right) \otimes \mathbb{I}_{n^{k-1}} \otimes a\right) \in M_{n^{2 k}},
$$

$\sigma_{k}$ a state on $M_{r(k)} \otimes_{\max } \mathcal{A}$ and $\phi_{k}: M_{n^{k}} \otimes S \rightarrow M_{n^{k}} \otimes M_{r(k)}$ a complete positive unital map, with $r(k)$ some positive integer. The norm $d_{c b}$ in equation (6.7) is the completely bounded norm. We used the identifications $\mathbb{I}_{n^{k}} \otimes s \in M_{n^{k-1}} \otimes M_{n} \otimes S=M_{n^{k}} \otimes S$ and likewise $\mathbb{I}_{n^{k-1}} \otimes a \in M_{n^{k}} \otimes \mathcal{A}$. Denoting by $j_{k}: M_{n} \rightarrow M_{n^{k}}$ the completely positive unital map $j(x)=x \otimes \mathbb{I}_{n^{k-1}}$ and by $j_{k}^{*}: M_{n^{k}} \rightarrow M_{n}$ its adjoint, we obtain from (6.7)

$$
d_{c b}\left(T, j_{k}^{*} \circ R_{k} \circ j_{k}\right) \leq \varepsilon .
$$

It follows that the state $\omega$ is well approximated by the completely positive map $\phi_{k}$ (after suitable extension to higher matrix levels by tensoring the identity) followed by application of the state $j_{k}^{*} \circ \sigma_{k} \circ j_{k}$ on $M_{r(k)} \otimes_{\max } \mathcal{A}$. For each sequence of positive integers $r(k)$ we may embed $M_{r(k)}$ into $\mathcal{B}\left(\ell_{2}(\mathbb{Z})\right)$, and furthermore we have that this embedding is complemented, i.e. there exists a completely positive map from $\mathcal{B}\left(\ell_{2}(\mathbb{Z})\right)$ onto $M_{r(k)}$ being equal to the identity when restricted to the matrix algebra in question. A short verification shows that we can replace the maps $\phi_{k}$ with maps into $\mathcal{B}\left(\ell_{2}(\mathbb{Z})\right)$, and the state $\sigma_{k}$ with states on $\mathcal{B}\left(\ell_{2}(\mathbb{Z})\right)$, having equivalent properties. We now furthermore demand that the "encoding map" $\phi_{k}$ does not depend on the original state $\omega$, but only on $s$. Since the subspaces of the form $\operatorname{span} x($ resp. $\operatorname{span} a)$ are dense in $S($ resp. in $\mathcal{A})$, the requirements of definition 6.3.2 are verified. It follows that $S$ is QWEPCP. 


\section{Conclusions and Outlook}

Summary. We studied the relation between Connes' embedding problem, or equivalently Kirchberg's QWEP conjecture, and the existence of finite-dimensional approximations of bipartite quantum systems. We did this from two different perspective. First, we introduced and studied the operator system of local measurements, which allowed us to study the case of two independent observer performing measurements on some joint quantum system in an abstract way. Using this operator system, we obtained that Kirchberg's QWEP conjecture would imply a positive solution to Tsirelson's problem, which asks whether all correlation functions corresponding to commuting measurements can also be expressed assuming that the underlying two-party Hilbert space is of tensor product form. In addition, we showed that if some observable algebra is QWEP, then Tsirelson's problem, subjected to measurements on quantum systems modeled by the observable algebra in question, has a positive answer, independent of the answer to Kirchberg's conjecture. We noted that the operator algebras having QWEP constitute a large class of physical examples. We then introduced a possible variant of the QWEP notion for the class of operator systems, studied its properties and also applied the results to the operator system of local measurements. Moreover, we elaborated on the connection of this variant of QWEP and the existence of finite-dimensional approximations for quantum systems, and compared our requirements with existing results from algebraic quantum field theory. In order to study the implications of the existence of certain kinds of approximations for general operator systems, we introduced the concept of ultraproducts in the category of operator systems. After some preliminary work on general concepts, we applied our findings to two different kinds of approximation assumptions, both motivated from quantum information theoretic considerations, and discussed their relation to the concepts defined in the previous chapters.

Future directions. The obvious open question is whether Connes' embedding problem has a positive answer. As outlined in this thesis, this would imply that in most information theoretic applications, there is no observable difference between quantum systems with a finite or infinite number of degrees of freedom. As a consequence, a possible negative answer to Connes' embedding problem might lead us to the conclusion that either sometimes an 
infinite amount of information is necessary, or the axioms of quantum mechanics might have to be strengthened in order to exclude these exotic cases. We hope that our reformulations in terms of concepts known from quantum information theory could lead to some further insight into these questions. 


\section{Bibliography}

[1] E. M. Alfsen, Compact convex sets and boundary integrals, Springer-Verlag, New York, 1971. Ergebnisse der Mathematik und ihrer Grenzgebiete, Band 57.

[2] C. Anantharaman-Delaroche, On ergodic theorems for free group actions on noncommutative spaces, Probab. Theory Related Fields, 135 (2006), pp. 520-546.

[3] H. Araki And E. Woods, A classification of factors., Publ. Res. Inst. Math. Sci., Kyoto Univ., Ser. A, 4 (1968), p. 51.

[4] W. B. Arveson, Subalgebras of $C^{*}$-algebras, Acta Math., 123 (1969), pp. 141-224.

[5] H. Barnum, J. Barrett, M. Leifer, and A. Wilce, Generalized no-broadcasting theorem, Phys. Rev. Lett., 99 (2007), p. 240501.

[6] H. Barnum AND A. Wilce, Information processing in convex operational theories, Preprint, arXiv:0908.2352 (2009).

[7] J. BARRETT, Information processing in generalized probabilistic theories, Phys. Rev. A, 75 (2007), p. 032304.

[8] J. S. Bell, On the Einstein-Podolsky-Rosen paradox, Physics, 1 (1964), pp. 194-200.

[9] — On the problem of hidden variables in quantum mechanics, Rev. Mod. Phys., 38 (1966), pp. 447-452.

[10] F. BocA, Free products of completely positive maps and spectral sets, J. Funct. Anal., 97 (1991), pp. 251-263.

[11] M. Bożejko, Positive-definite kernels, length functions on groups and a noncommutative von Neumann inequality, Studia Math., 95 (1989), pp. 107-118.

[12] N. P. Brown, Connes' embedding problem and Lance's WEP, Int. Math. Res. Not., (2004), pp. 501-510.

[13] D. Buchholz, C. D'Antoni, and K. Fredenhagen, The universal structure of local algebras, Comm. math. Phys., 111 (1987), pp. 123-135.

[14] V. Capraro, A Survey on Connes' Embedding Conjecture, Preprint, arXiv:1003.2076 (2010).

[15] M. D. Choi And E. G. Effros, Injectivity and Operator Spaces, J. Func. Ana., 24 (1977), p. 156. 
[16] J. Clauser, M. Horne, A. Shimony, and R. Holt, Proposed experiment to test local hidden-variable theories, Phys. Rev. Lett., 23 (1969), p. 880.

[17] A. Connes, Classification of injective factors. Cases $I I_{1}, I I_{\infty}, I I I_{\lambda}, \lambda \neq 1$, Ann. of Math. (2), 104 (1976), pp. 73-115.

[18] D. Dacunha-Castelle and J. L. Krivine, Applications des ultraproduits à l'étude des espaces et des algèbres de banach, Studia Math., 41 (1972), pp. 315-334.

[19] J. DE PILLIS, Linear transformations which preserve hermitian and positive semidefinite operators, Pacific J. Math., 23 (1967), pp. 129-137.

[20] J. Diestel and J. Uhl, Vector measures, Mathematical surveys, American Mathematical Society, 1977.

[21] K. Dykema And K. Juschenko, Matrices of unitary moments, Preprint, arXiv:0901.0288v2 (2009).

[22] E. G. Effros, M. Junge, And Z.-J. Ruan, Integral Mappings and the Principle of Local Reflexivity for Noncommutative $L_{1}$-Spaces, Ann. of Math., 151 (2000), p. 59.

[23] E. G. Effros and E. C. Lance, Tensor products of operator algebras, Adv. Math., 25 (1977), pp. 1-34.

[24] E. G. Effros And S. Winkler, Matrix convexity: Operator analogues of the bipolar and Hahn-Banach theorems, J. Funct. Anal., (1997).

[25] I. Farah, B. Hart, And D. Sherman, Model theory of operator algebras I: Stability, Preprint, arXiv:0908.2790v2 (2009).

[26] D. Farenick and P. B. Morenz, $C^{*}$-Extreme Points of some Compact $C^{*}$-Convex Sets, Proc. Amer. Math. Soc., 118 (1993), p. 765.

[27] D. Farenick and V. I. Paulsen, Operator system quotients of matrix algebras and their tensor products, Preprint, arXiv:1101.0790v1 (2011).

[28] T. Franz, F. Furrer, And R. F. Werner, Extremal quantum correlations and cryptographic security, Phys. Rev. Lett., 106 (2011), p. 250502.

[29] T. FRITZ, Operator system structures on the unital direct sum of $C^{*}$-algebras, Preprint, arXiv:1011.1247v2 (2010).

[30] — Tsirelson's problem and kirchberg's conjecture, Preprint, arXiv:1008.1168v3 (2010).

[31] L. Ge And D. Hadwin, Ultraproducts of $C^{*}$-algebras, Recent advances in operator theory and related topics, 127 (2001), p. 305.

[32] U. GROH, Uniform ergodic theorems for identity preserving schwarz maps on $w^{*}$ algebras, J. Operator Theory, 11 (1984), pp. 395-404.

[33] A. Grothendieck, Produits tensoriels topologiques et espaces nucleaires., Mem. Am. Math. Soc., 16 (1955), p. 150 p. 
[34] R. HaAg, On quantum field theories, Danske Vid. Selsk. Mat.-Fys. Medd, 29 (1955), p. 37.

[35] R. HaAg, Local Quantum Physics: Fields, Particles, Algebras, Springer, New York, 1992.

[36] U. HaAgerup, A new proof of the equivalence of injectivity and hyperfiniteness for factors on a separable hilbert space, J. Funct. Anal., 62 (1985), pp. 160-201.

[37] U. HaAgerup and M. Musat, Factorization and dilation problems for completely positive maps on von neumann algebras, Comm. Math. Phys., 303 (2011), pp. 555-594. 36 pages.

[38] K. H. Han, Tensor products and nuclearity of ordered vector spaces with archimedean order unit, Preprint, arXiv:0906.1858v1 (2009).

[39] — - On maximal tensor products and quotient maps of operator systems, J. Math. Anal. Appl., (2011).

[40] K. H. Han And V. I. PAUlsen, An approximation theorem for nuclear operator systems, Preprint, arXiv: 1009.2541v2 (2010).

[41] L. Hardy, Quantum Theory From Five Reasonable Axioms, Preprint, arXiv:quant$\mathrm{ph} / 0101012(2001)$.

[42] S. HeInRICH, Ultraproducts in banach space theory, J. Reine Angew. Math., 313 (1980), pp. $72-104$.

[43] A. Jamiolkowski, Linear transformations which preserve trace and positive semidefiniteness of operators, Rep. Math. Phys., 3 (1972), pp. 275 - 278.

[44] M. Junge, Fubini's Theorem for Ultraproducts of Noncommutative $L_{p}$-Spaces, Canad. J. Math., 56 (2004), pp. 983-1021.

[45] M. Junge, M. Navascues, C. Palazuelos, D. Perez-Garcia, V. B. Scholz, AND R. F. Werner, Connes' embedding problem and Tsirelson's problem, J. Math. Phys., 52 (2011).

[46] M. Junge and C. Palazuelos, Large violation of bell inequalities with low entanglement, Comm. math. Phys., 306 (2011), pp. 695-746.

[47] M. Junge, C. Palazuelos, D. Pérez-García, I. Villanueva, and M. Wolf, Operator spaces: a natural language for bell inequalities, Phys. Rev. Lett., 104 (2010), p. 170405.

[48] — Unbounded violations of bipartite bell inequalities via operator space theory, Commun. Math. Phys., 300 (2010), pp. 715-739.

[49] K. Juschenko And S. Popovych, Algebraic reformulation of connes embedding problem and the free group algebra, Israel J. Math., 181 (2011), pp. 305-315.

[50] A. S. KaVRuk, Nuclearity related properties in operator systems, Preprint, arXiv:1107.2133v4 (2011). 
[51] A. S. Kavruk, V. I. Paulsen, I. G. Todorov, and M. Tomforde, Tensor products of operator systems, Preprint, arXiv:0910.2210 (2009).

[52] A. S. Kavruk, V. I. Paulsen, I. G. Todorov, and M. Tomforde, Quotients, exactness, and nuclearity in the operator system category, Preprint, arXiv:1008.2811v2 (2010).

[53] M. Keyl, T. Matsui, D. Schlingemann, and R. F. Werner, Entanglement, Haag-duality and type properties of infinite quantum spin chains, Rev. Math. Phys, 18 (2006), p. 935.

[54] M. Keyl, D. Schlingemann, And R. F. Werner, Infinitely entangled states, Quant. Inf. Comp., 3 (2003), p. 281.

[55] E. KirchberG, On non-semisplit extensions, tensor products and exactness of group $C^{*}$-algebras, Invent. Math., 112 (1993), pp. 449-489.

[56] I. Klep And M. Schweighofer, Connes' embedding conjecture and sums of hermitian squares, Adv. Math., (2008).

[57] E. C. Lance, On Nuclear C*-algebras, J. Func. Ana., 12 (1973), p. 157.

[58] R. I. Loebl, Injective von Neumann Algebras, Proc. Amer. Math. Soc., 44 (1974), pp. pp. $46-48$.

[59] G. Ludwig, Foundations of Quantum mechanics I, Springer, New York, 1983.

[60] — _ Foundations of Quantum Mechanics II, Springer, New York, 1985.

[61] L. Masanes and M. P. Mueller, A derivation of quantum theory from physical requirements, Preprint, arXiv:1004.1483 (2010).

[62] D. McDuff, Central sequences and the hyperfinite factor, Proc. London Math. Soc. (3), 21 (1970), pp. 443-461.

[63] P. Mittelstaedt, The Interpretation of Quantum Mechanics and the Measurement Process, Cambridge University Press, 2004.

[64] F. J. Murray and J. von Neumann, On Rings of Operators. IV, Ann. of Math., 44 (1943), pp. pp. 716-808.

[65] I. Namioka And R. Phelps, Tensor products of compact convex sets., Pac. J. Math., 31 (1969), pp. 469-480.

[66] M. Navascues, S. Pironio, And A. Acin, Bounding the set of quantum correlations, Phys. Rev. Lett., 98 (2007), p. 010401.

[67] M. A. Nielsen And I. L. ChuAng, Quantum Computation and Quantum Information (Cambridge Series on Information and the Natural Sciences), Cambridge University Press, 2004.

[68] O.Bratteli and D. W. Robinson, Operator Algebras and Quantum Statistical Mechanics 2, Springer, 1996. 
[69] N. OzawA, About the QWEP conjecture, Internat. J. Math., 15 (2004), pp. 501-530.

[70] V. Paulsen, Completely Bounded Maps and Operator Algebras, vol. 78 of Cambridge Studies in Advanced Mathematics, Cambridge University Press, 2002.

[71] V. I. Paulsen, I. G. Todorov, and M. Tomforde, Operator system structures on ordered spaces, Preprint, arXiv:0904.3783v1 (2009).

[72] V. I. Paulsen And M. Tomforde, Vector spaces with an order unit, Preprint, arXiv:0712.2613v4 (2007).

[73] G. PISIER, A simple proof of a theorem of Kirchberg and related results on $C^{*}$-norms, J. Operator Theory, 35 (1996), pp. 317-335.

[74] G. PISIER, Introduction to operator space theory, vol. 294 of London Mathematical Society Lecture Note Series, Cambridge University Press, Cambridge, 2003.

[75] M. B. Plenio And S. Virmani, An introduction to entanglement measures, Quant.Inf.Comput., 7 (2007), pp. 1-51.

[76] I. Raeburn And A. M. Sinclair, The $C^{*}$-algebra generated by two projections, Math. Scand., 65 (1989), p. 278.

[77] Y. Raynaud, On ultrapowers of non commutative $L_{p}$ spaces, J. Operator Theory, (2002).

[78] M. Rørdam And E. Størmer, Classification of Nuclear $C^{*}$-algebras, vol. 7 of Operator Algebras and Non-Commutative Geometry, Springer, 2002.

[79] Z.-J. RuAn, Subspaces of $C^{*}$-algebras, J. Funct. Anal., 76 (1988), pp. 217-230.

[80] H. Schaefer And M. WolfF, Topological vector spaces, Graduate texts in mathematics, Springer, 1999.

[81] V. B. Scholz, On the existence of local hidden variable models for two-quibt quantum states, Master's thesis, University of Erlangen-Nürnberg, 2007.

[82] J. A. Smolin, F. Verstraete, And A. Winter, Entanglement of assistance and multipartite state distillation, Phys. Rev. A, 72 (2005), p. 052317.

[83] M. TAKesaki, Theory of operator algebras. I, vol. 127 of Encyclopaedia of Mathematical Sciences, Springer-Verlag, Berlin, 2003.

[84] — Theory of operator algebras. II, vol. 127 of Encyclopaedia of Mathematical Sciences, Springer-Verlag, Berlin, 2003.

[85] — Theory of operator algebras. III, vol. 127 of Encyclopaedia of Mathematical Sciences, Springer-Verlag, Berlin, 2003.

[86] B. S. Tsirelson, Some results and problems on quantum Bell-type inequalities, Hadronic J. Suppl., 8 (1993), p. 329. 
[87] —, Bell inequalities and operator algebras, Hannover list of open problems in quantum information theory, http://qig.itp.uni-hannover.de/qiproblems/ Bell_inequalities_and_operator_algebras (2006).

[88] C. Webster And S. Winkler, The Krein-Milman theorem in operator convexity, Trans. Amer. Math. Soc., 351 (1999), pp. 307-322.

[89] R. F. WERnER, Quantum states with Einstein-Podolsky-Rosen correlations admitting a hidden-variable model, Phys. Rev. A, 40 (1989), pp. 4277-4281.

[90] R. F. Werner And M. M. Wolf, Bell inequalities and entanglement, Quantum Inf. Comput., 1 (2001), pp. 1-25.

[91] P. ZANARDi, D. LidAR, AND S. LlOyd, Quantum tensor product structures are observable-induced, Phys. Rev. Lett., 92 (2004), p. 060402. 


\section{VOLKHER BERNHARD SCHOLZ}

Institute for Theoretical Physics

Leibniz Universität Hannover

30167 Hannover, Germany

volkher.scholz@itp.uni-hannover.de

\section{PERSONAL INFORMATION}

Born August 12, 1983 in Siegen, Germany.

German citizen.

Single.

Languages: German, English.

\section{RESEARCH TOPICS}

Quantum information theory for systems with infinitely many degrees of freedom, especially quantum communication in the setup of von-Neumann algebras and transport properties of quantum lattice systems.

\section{EDUCATION}

October 2003 - March 2007

Diploma of physics at the Friedrich Alexander Universität ErlangenNürnberg, Germany. Thesis: On the existence of local-hidden-variable models for twoqubit quantum states, Supervised by Prof. Hajo Leschke and Prof. Norbert Lütkenhaus.

May 2006 - November 2006

Visiting student in the group of Norbert Lütkenhaus at the Institute of Quantum Computing, University of Waterloo, Ontario, Canada.

September 1994 - June 2003

Allgemeine Hochschulreife, Ehrenbürg Gymnasium Forchheim and Gymnasium Fränkische Schweiz Ebermannstadt, Germany

\section{EMPLOYMENT HISTORY}

since April 2009

Research assistant at the Institute for Theoretical Physics, Leibniz Universität Hannover, Germany.

November 2007 - April 2009

Research assistant at the Institute for Mathematical Physics, Technische Universität Braunschweig, Germany. 
AWARDS \& SCHOLARSHIPS

April 2007

Prize of the faculty of physics and mathematics, Friedrich Alexander Universität Erlangen-Nürnberg, for my Diploma thesis.

$2005-2007$

Stipend of the "Studienstiftung des Deutschen Volkes".

\section{TEACHING}

\section{since April 2009}

Tutorials on the mathematics of Anderson localization, ordered vector spaces, operator systems and related topics. Teaching assistant for the lecture "Quantensprünge". Involved in the supervision of several bachelor students, all in connection with my employment at Institute for Theoretical Physics, Leibniz Universität Hannover, Germany.

November 2007 - April 2009

Teaching assistant for the student seminar "entropy" at the Institute for Mathematical Physics, Technische Universität Braunschweig, Germany.

April 2007 - November 2007

Teaching assistant for the course on computational physics at the Institute for Theoretical Physics, Friedrich Alexander Universität Erlangen-Nürnberg, Germany.

\section{PROFESSIONAL ACTIVITIES}

\section{Grant applications}

Extensive involvement in the administration of german and european research projects. This includes contributing to grant proposals and annual reports ( projects SCALA and COQUIT ), as well as giving presentations at annual review meetings ( project COQUIT )

Organization of workshops.

Co-organizer of the workshop "Reisensburg workshop on quantum information theory", Reisensburg, May 2010. Co-organizer of the first COQUIT workshop, Hannover, 2010.

Other administrative activities.

Extensively involved in the administration activities of the quantum information theory group in Hannover which includes being on the committee for job applications, organizing seminars and taking care of the technical equipment. 
Tsirelson's problem and the Connes' embedding conjecture, Institute Mittag Leffler seminar, Djursholm, Sweden, September 2010.

Tsirelson's problem and Kirchberg's QWEP conjecture, Workshop on Noncommutative Lp-Spaces, Operator Spaces and Applications, Banff International Research Station, July 2010.

Quantum Walks - Disorder in space and time, IQG Physics Lunch Meeting, University of Waterloo, June 2010.

Tutorial on the mathematics of Anderson Localization, Imperial College London, July 2009.

A short note Tsirelson's problem, Workshop on Operator structures in Quantum information, Fields institute, Toronto, July 2009 (Contributed talk).

Tensor norms and Tsirelson's problem, Information primitives and laws of nature, Zurich, May 2008.

I have also given seminar talks at places including the Californian Institute of Technology, the TU in Munich, the ETH in Zurich and the Universities of Innsbruck, Düsseldorf, Würzburg and Erlangen.

See further up. 